

\section{Published by Peertechz Publications}

Title: Regeneration of the human spinal cord via coordination dynamics therapy

Subjects: Orthopedics and Rheumatology

Received: 22-05-2019 | Accepted: 05-08-2019 | Published: 0608-2019

*Corresponding author: Giselher Schalow, Private Researcher, Untere Kirchmatte 6, CH-6207 Nottwil, Switzerland, E-mail: g_schalow@hotmail.com

ISBN: 978-81-943057-0-5

DOI: 10.17352/ebook10111 


\section{Table of Contents}

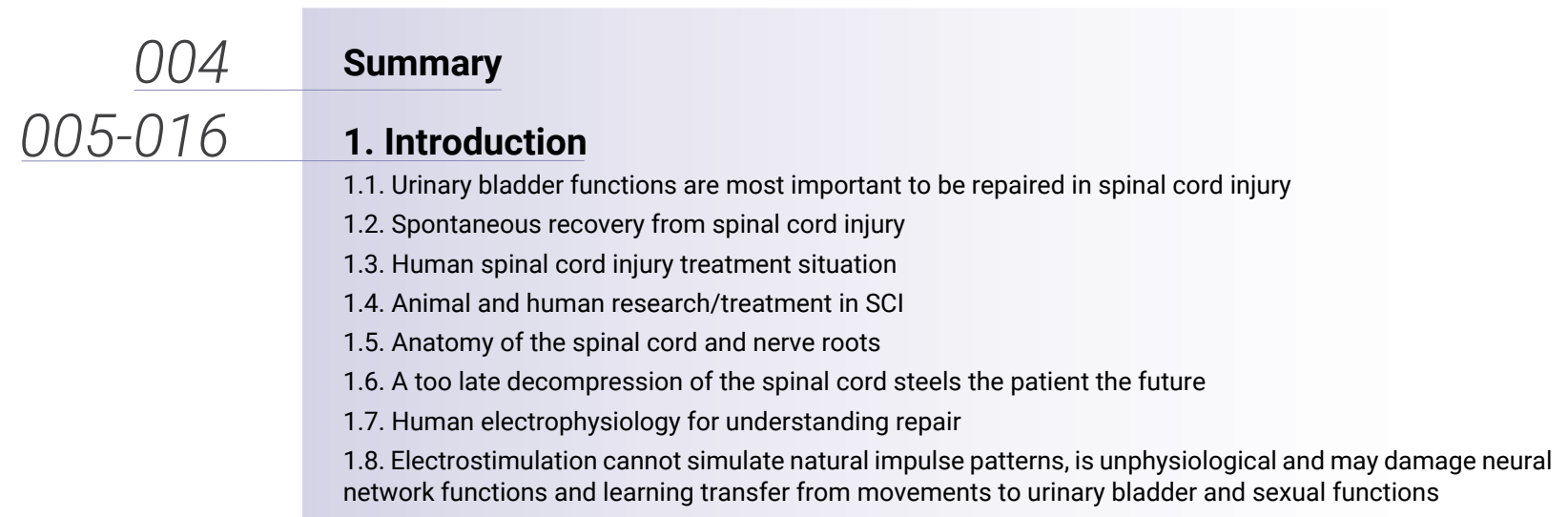

\section{$017-023 \quad$ 2. Method}

2.1. Coordination dynamics therapy

2.2. System Theory of Pattern Formation for understanding neuronal network organization and learning and measure repair progress

2.3. Learning implications for treatment derived from the equations of motion of the collective variables

2.4. Geographical landscape of attractors

2.5. Quantifying the improvement of CNS functioning by measuring pattern stability upon pattern change when exercising on the special CDT device

\section{4-085 3. Results}

3.1. Review of spinal cord injury repair via coordination dynamics therapy

3.2. Regeneration of the human spinal cord during development

3.3. Case report of spinal cord repair $(70 \% \mathrm{SCl})$ in the patient Nefeli via coordination dynamics therapy (CDT) up to 1 year

3.4. Spinal cord injury repair within 2 years

3.5. Spinal cord repair within 2.5 years

3.6. Spinal cord repair within 3 years

3.7. Spinal cord repair within 3.5 years of therapy (September 2018)

\section{6-093 4. Discussion}

4.1. Limitations of basic science research

4.2. A need for scientific and clinical human approach

4.3. Animal experiments are removed from human reality

4.4. Consequences of false hope

4.5. Ethics of SCl research, treatment, and clinical trials - false hope from animal treatment research

4.6. Out-of-date of the clinical treatment system

4.7. Children with $\mathrm{SCl}$ are hoping for better treatment to become like the other children again

4.8. Ethics to use the patients name

\section{4-097 5. References}




\section{Summary}

In the Introduction, the human neurophysiology is introduced for repairing spinal cord injuries. Based on the recordings with a new electrophysiologic method, the single-nerve fiber action potential recording method, and morphometry of nerve roots, a classification of human peripheral nerve fibers is developed and used for the identification of neurons from which was recorded to analyze spinal cord functions. Simultaneous impulse patterns of several single neurons, evoked by natural stimulations, running in and out of the spinal cord, are used to analyze spinal cord functions under rather physiologic and pathologic conditions. By combining the electrophysiology with the "System Theory of Patterns Formation", the movementbased learning method "Coordination Dynamics Therapy" (CDT) is developed to repair the human spinal cord. In the first part of the Results, the achieved repairs in spinal cord injury (SCI) through CDT are given in a group of patients and the single cases of $50 \%$ and $95 \%$ cervical SCI. In 50\% SCI, urinary bladder functions, walking and running were repaired. In 95\% SCI, bladder functions and trunk control were repaired, but free walking was not achieved. In the second part, first time in history, the regeneration of the human spinal cord of the 10-year-old Nefeli was measured by the re-innervation of segment-indicating muscles below the injury level of Th10. It turned out that most of the approximate one-year lasting regeneration time was needed to cross the injury site. Then, the fastest nerve fibers regenerated with a speed of $1 \mathrm{~mm} /$ day down to the muscles. In the third part, details of the case report to repair the spinal cord by movement-based learning are given. Emphasis is put on the repair of the urinary bladder function, because its repair is most important for patients with SCI. In the Discussion, the deficits of animal research and clinics are given. Possible reasons for the regeneration of the human spinal cord may be that the power of regeneration is higher below an age of 10 and efficient aggressive treatment has to be administered continuously for more than one year. A permanent coma patient started to speak through 6 years of CDT with 20 hours efficient therapy per week. 


\section{Introduction}

\subsection{Urinary bladder functions are most important to be repaired in spinal cord injury}

Before World War II, most patients with a spinal cord injury (SCI) died because of recurrent urinary tract infections. Nowadays, in India the life expectancy time of a patient with a severe SCI, after leaving the clinic, is 1 year because at home bladder and other infections cannot be managed sufficiently. Needless to say, that one of the most important functions to be repaired in patients with SCI is the control of the urinary bladder, followed by the sexual function, walking, spasticity, pressure ulcers and other functions. Most functions below the injury level (Figure 1) are lost or impaired.

\section{Spinal cord segments in relation to the vertebral bodies, dermatome} segmental innervation and level of spinal cord injury

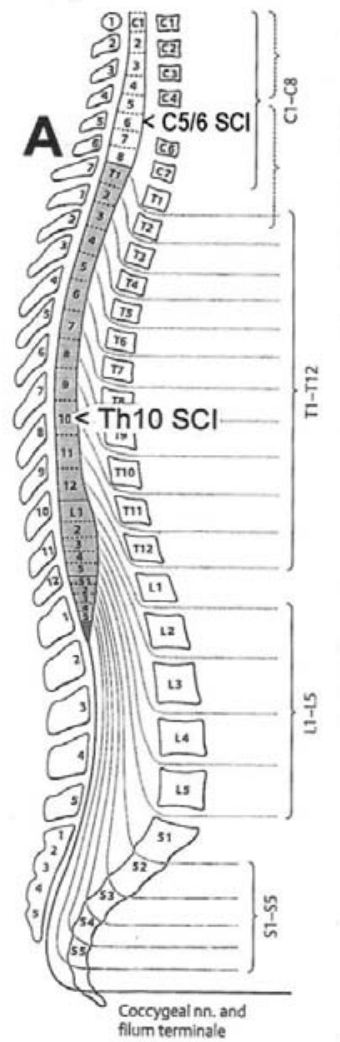

B
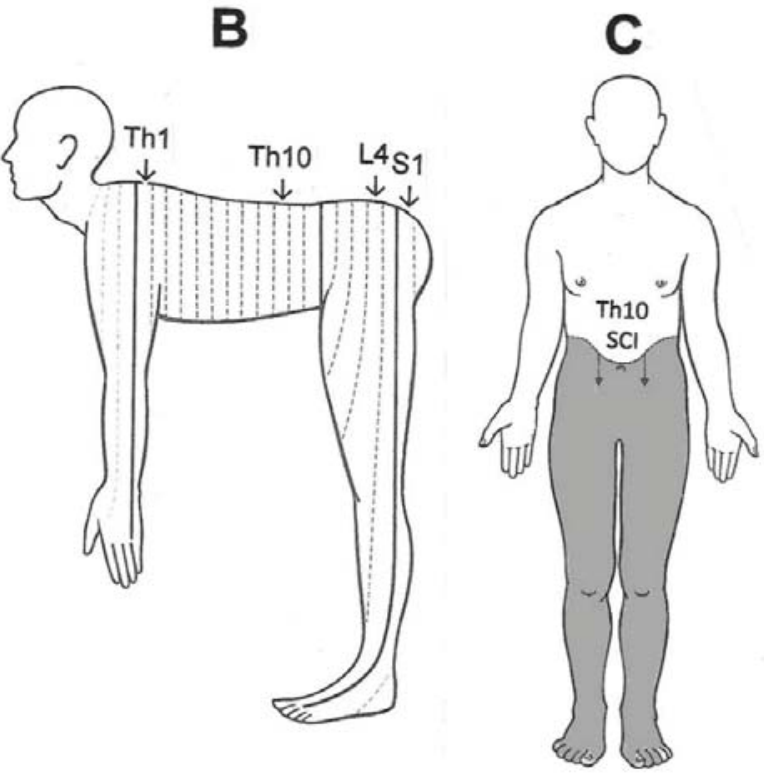

Figure 1: A. Spinal cord segments and their relation to the vertebral bodies. Note the Ascensus of the spinal cord, giving rise to the long cauda equina nerve roots. B. Approximate segmental innervation of the skin. C. Below the SCI level there is loss of sensitivity and loss of connectivity to muscle and other functions. 
Patients are often reluctant to undergo urodynamic evaluation in a rehabilitation center, because of the fear of contracting urinary tract infection with antibiotic resistant bacteria. Development of urinary tract infection is a major set-back to the therapy, as optimal therapy is not possible in the presence of such an infection. If urodynamic assessment is not available, some good practical indicators of improvement in urinary bladder function are the reduction in the incidence of urinary bladder infections and the significant reduction in the volume of post-voidal residual urine in the bladder. Residual urine can be measured by emptying the bladder with a catheter, after the patient has passed urine voluntarily. Residual urine of less than $50 \mathrm{ml}$ indicates good physiological functioning of the bladder.

After World War II, Guttmann from Breslau [1], introduced intermitted catheterization to keep SCI patients alive. The neurosurgeon L.W. Freeman (USA) stated in the 1960's after performing very many SCI repair experiments on dogs, "it is difficult to find persons who do research in paraplegia and to get money for such research; probably World War II is already too far away". By cutting only one half of the spinal cord in animal experimentation, one is not getting urinary bladder and skin nutrition problems, but the repair in a $50 \%$ SCI is already solved in human [2], (Figure 19G,H).

\subsection{Spontaneous recovery from spinal cord injury}

In incomplete SCI (Figure 19D), the patients can also re-learn running and jumping (Figure 19G,H), besides proper breathing and urinary bladder control and can improve cardio-vascular performance. Such a repair can be mainly achieved by a functional reorganization. In almost complete cervical SCI, there is nearly no spontaneous recovery. In incomplete SCI there is some spontaneous recovery, especially during the following weeks after the spinal shock. Most spontaneous recovery occurs in the first 6 months following the injury. One year after the injury there occurs normally no further spontaneous recovery. Conventional rehabilitation centers, which are concerned with care and not with cure, 'sell' the mainly spontaneous recovery as treatment-induced repair. In a case report it was explained how an 18-year-old boy, who suffered an incomplete SCI at the cervical 5/6 segments, could achieve a near-complete recovery within 3 years of CDT and improved further in the following 3 years [2].

\subsection{Human spinal cord injury treatment situation}

For judging SCI repair, one needs to know how much percent of the spinal cord was damaged, what treatment was used and the improvement of functions with treatment has to be quantified objectively. SCI's are known for hundreds of years and all easy treatments will have been tried out, including electrostimulation and interponats placed into the injured spinal cord site to re-connect the cord. The main drawing back in repair is that the injured adult spinal cord does not regenerate spontaneously. Placing a piece of nerve to the injury site to reconnect the proximal and distal spinal cord parts will not help, because the human nerve fibers cannot grow from the cord into the nerve transplant and vice versa. In rat, nerve fibers of the cord can cross a gap up to $12 \mathrm{~mm}$. By using an olfactory transplant with stem cells, may be some fibers can cross the transplanted site, but the damage to the still existing fibers is probably higher than the gained new connections, because the injured spinal cord is very vulnerable. The only successful treatment so far is the CDT [3], as is shown here. The nervous system is functionally repaired by movement-based learning, including some regeneration. In this paper it is reported about the enhancement of the limited regenerative capacity of the human spinal cord. Some details of human anatomy and human electro-physiology are given to make the reader understandable on what level of research treatment has to be designed to be successful on the long term. A combination of CDT and stem-cell therapy should be tried in the future. "The medical promise of stem cells remains real, but largely unrealized for now. The excitement must not be left to dissolve into a muddle of disappointment, frustration and fear because of the practices of a few irresponsible profiteers" [4].

\subsection{Animal and human research/treatment in SCI}

It has been reported that SCI is a devastating condition for which there is as yet no cure $[5,6]$, even though it has been published that SCI can partly be repaired through CDT [2,7-9]. A century step forward 
was that also in a motoric complete cervical SCI, which is really a devastating condition when there are nearly no motor functions remained, the most important urinary bladder function could be repaired and this repair included some regeneration of the human spinal cord besides a functional repair [9].

Basic scientific research (including human research) should provide a rational basis for tailoring specific combinations of therapies [5]. Reviewers cite human work without a scientific basis and argue then, that neuro-rehabilitation methods should have a scientific basis.

The main difference with respect to repair between animal and human is the power of regeneration and learning. In animals the nerve fiber growing strategy is used for repair because of the high regenerative capacity and in human the re-learning is used for functional reorganization because in human the regenerative capacity is small and the learning capacity high. Therefore, the animal research in SCI is bypassing the interests of human patients with SCI. But in animal and human, both, regeneration and learning are needed to a certain extent.

For sure: Specialist journals and general audience media need to set reasonable expectations of the safety and efficacy of potential therapies to avoid raising and then dashing the hopes of those living with SCI or those in government, those carrying out research, or the general public [5]. High ethical standards are required by researchers, clinicians and journalists to ensure that results are communicated to the general public in a manner that honestly reflects the safety and efficacy of a potential therapy [5].

\subsection{Anatomy of the spinal cord and nerve roots}

The level of a SCI is diagnosed by the segmental innervation of the skin (Figure 2) and the segmentindicating muscles (Figure 37), that means till what caudal skin-segment the patient can feel and till what caudal muscles he/she can activate on volition. There is some variation of the segmental innervation of the skin and the muscles.

Since in animal research and clinics original pictures of the human spinal cord and magnetic resonance images (MRI) are seldomly shown, it will be done here. Figure 3 shows the human spinal cord, dissected by the Author. It can be seen that the cauda equina nerve roots are much longer than the thoracic roots. In Figure $6 \mathrm{~A}$ the spinal cord is shown in connection with the brain. The cauda equina, after opening the dura mater, is shown in Figure 6C in a cadaver dissection and during an operation in Figure 6B.

The vascularization of the lower spinal cord and nerve roots can be seen in Figure 4. A compression of the spinal cord will not only damage the spinal cord tissue directly, but also indirectly by impairing its blood supply. The spinal cord is protected by the spinal canal and is hold in place in the spinal fluid via the denticulate ligaments (in Figure 4). When in an accident the spine brakes and presses onto the spinal cord (Figure 5A), the cord has to be decompressed as quickly as possible, not to increase the pressure damage with ongoing time. The decompression has to be performed within the 8 hours following the accident, especially in cervical SCI. Wrong handling during the transport of the injured patient has to be avoided, especially in cervical SCI, not to increase the damage. Often further spinal cord damage takes place by wrong transport and too late spine operations.

\subsection{A too late decompression of the spinal cord steels the patient the future}

Figure 5 demonstrates the tremendous consequences of a too late decompression of the spinal cord. Since repair is mainly achieved by functional reorganization of the brain, it is of importance to reduce secondary damage due to wrong handling and care and too late spinal cord decompression.

In Switzerland on a Sunday a motorcyclist suffered a SCI. In the hospital in 'Lausanne' the surgeons wanted to operate the patient immediately, probably within 8 hours after the accident to reduce quickly the spinal cord compression (Figure 5A). The mother wanted the best for her child and took her boy to a well- 


\section{Segmental innervation of the skin}

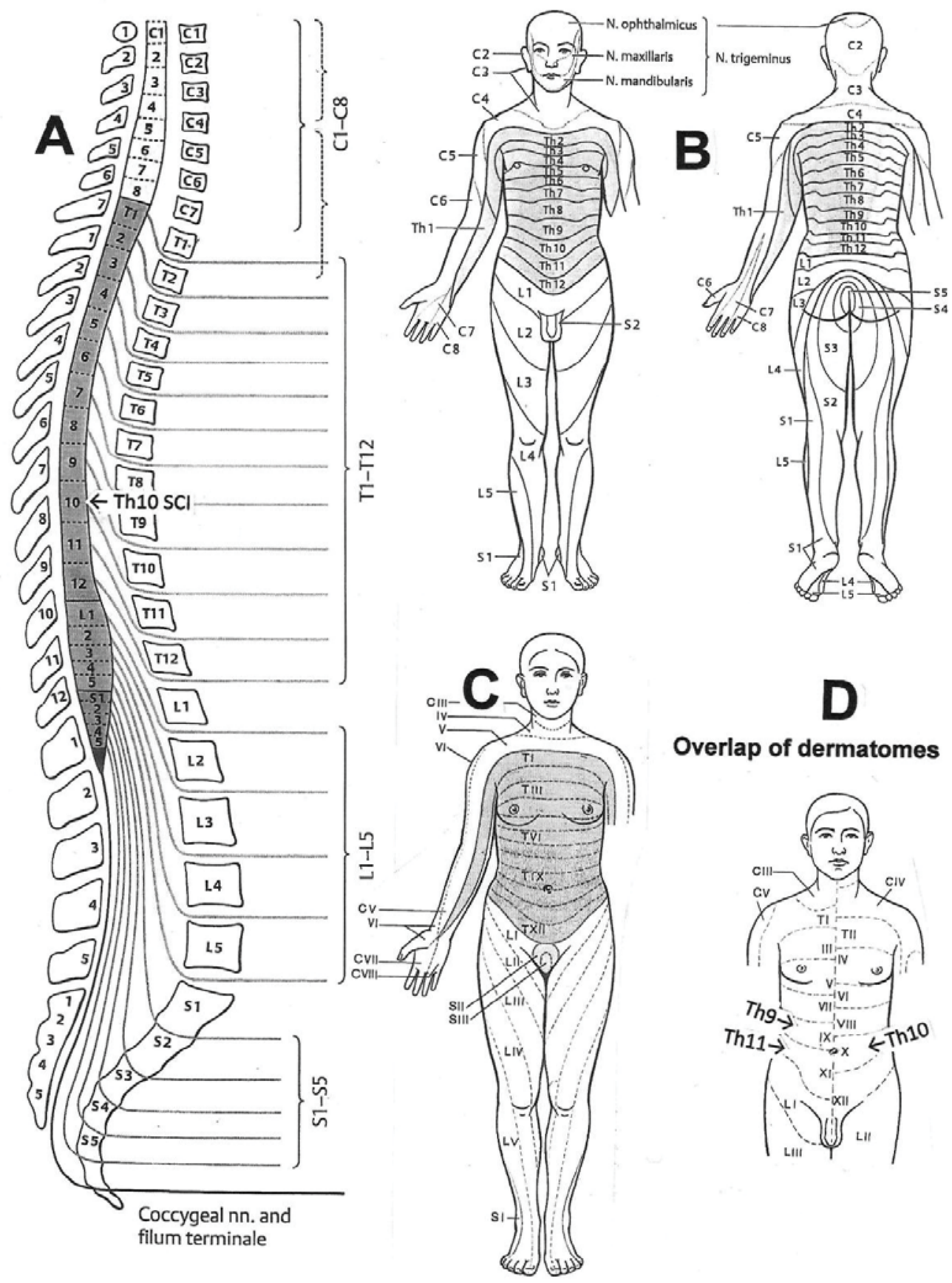

Figure 2: Spinal cord (A) and dermatome segments (B-D). The segmental innervation of the skin in B is according to Hansen-Schliack and in C after Keegan and Garret. The overlap of dermatomes (D) is according to Foerster. Note the different location of the T1 dermatome in B and C. There is variation of the segmental skin innervation, especially in the lower sacral range.

known rehabilitation center in Switzerland. The orthopedic surgeon of the rehabilitation center decided on the basis of the MRI of Figure 5A to postpone the operation to the Monday morning. One can see from the MRI of Figure 5A that there was already an injury of approximately $70 \%$. But by postponing the operation to the next day and leaving the pressure onto the cord the injury increased from the probable $70 \%$ to $99 \%$ (Figure 5B). Because of this additional loss of spinal cord matter, due to the late removal of the pressure 

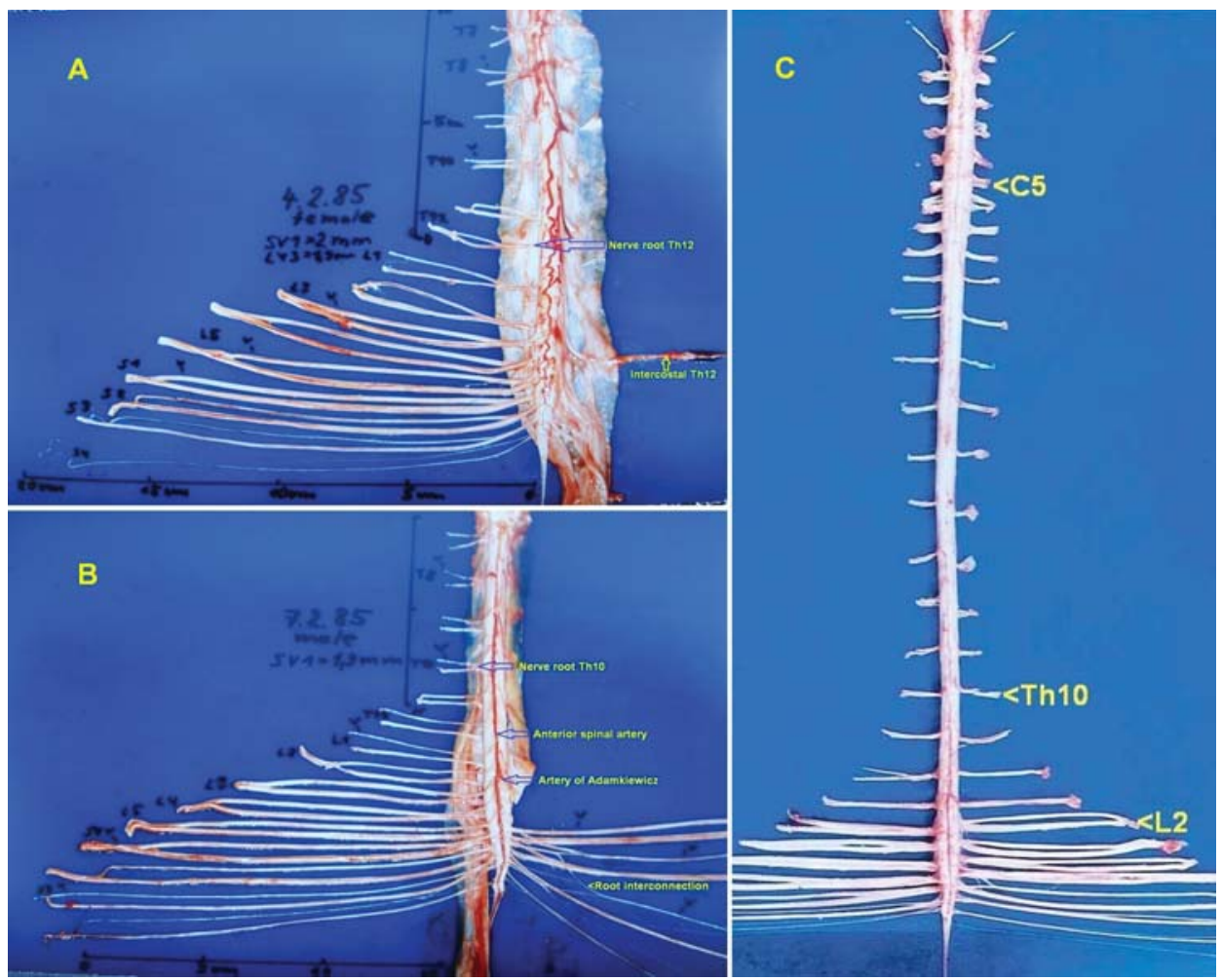

Figure 3: Human spinal cord from dorsal (A) and ventral (B,C). Intumescentia cervicalis and lumbosacralis are visible in C. The caudal ventral roots are thinner than the dorsal roots. The passage of the artery spinalis magna (Artery of Adamkiewicz) and the anterior spinal artery are indicated. The C5, Th10 and L2 roots and the intercostal nerve Th12 are indicated. Dissection by the Author.

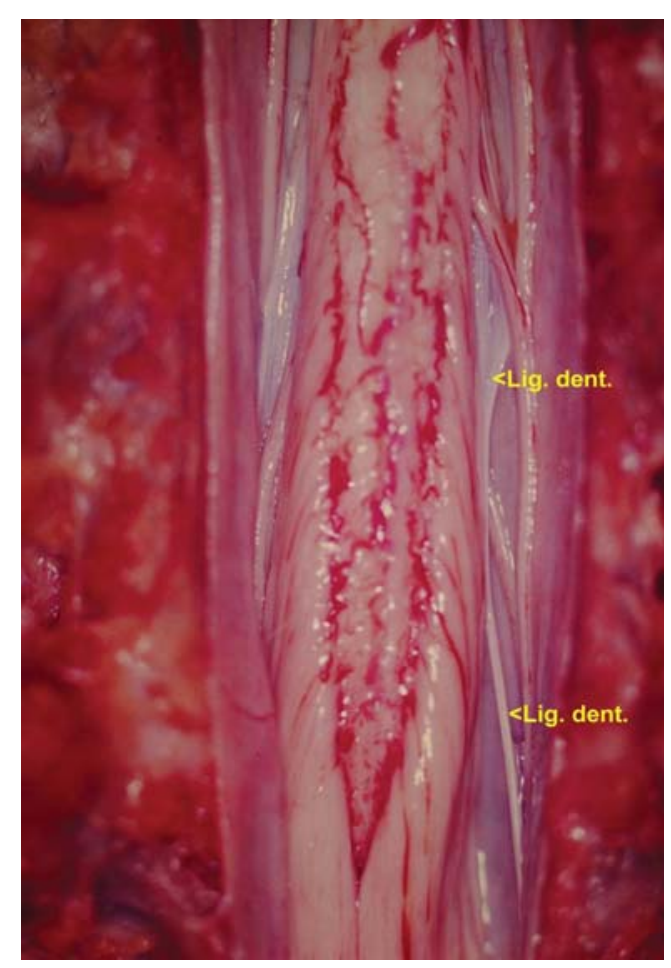

Figure 4: Caudal part of the human spinal cord from dorsal. Lig. dent.$=$ denticulate ligament. Dissectionv of the Author. exerted from the vertebras onto the cord, the 18 years old patient lost part of his quality of life for the rest of his life. It will be shown below that with an injury of $70 \%$ you can relearn walking and get most functions repaired below the injury level C5/6. Only hand and finger functions would be impaired because of the loss of the grey matter at the injury site $\mathrm{C} 5 / 6$, where motoneuron cell bodies are located.

\subsection{Human electrophysiology for understanding repair}

With the single-nerve fiber action potential recording method one can record simultaneously at the neuron level natural impulse patterns (Figure 6) running in and out of the spinal cord [10-15].

The impulse patterns of single-nerve fibers can be separated by splitting the multi-unit recording into impulse patterns of single-fibers by waveform comparisons, conduction times of single action potentials and impulse patterns. Figure 7 shows such a splitting of the impulse traffic of several fibers into the impulse patterns of single fibers. The multi-fiber recording of Figure 6 is split into the activity of single fibers. 

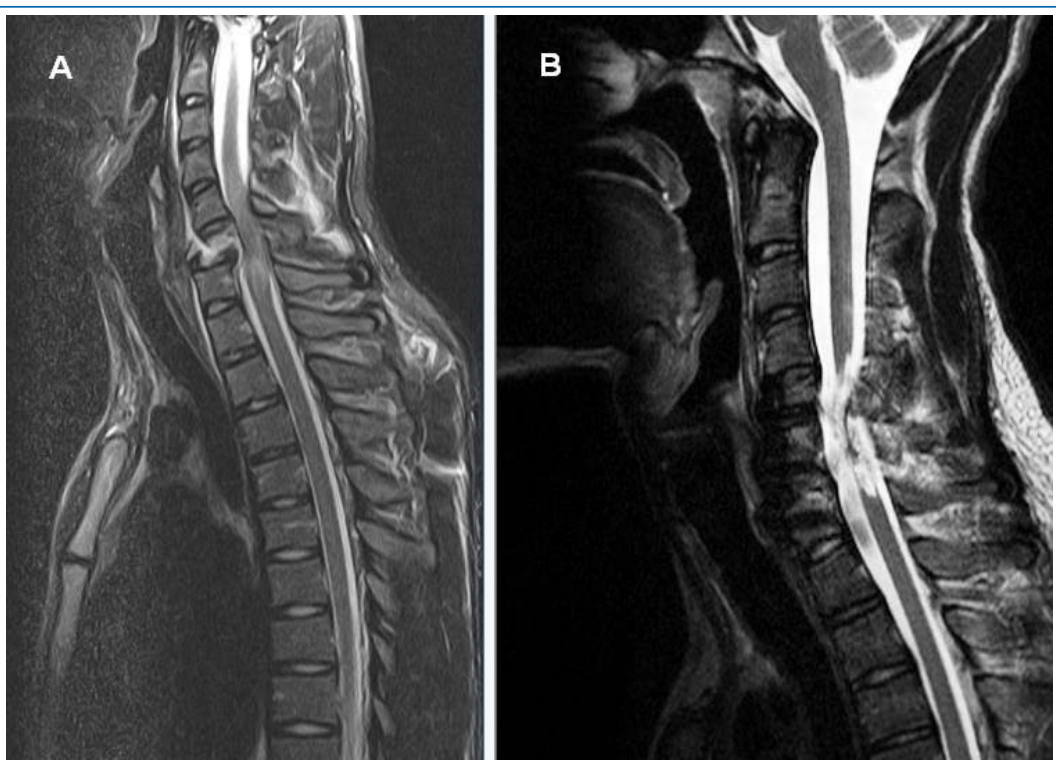

Figure 5: Cervical SCI of an 18-year old man approximately 8 hours after a motor cycle accident (A) and one year later (B).

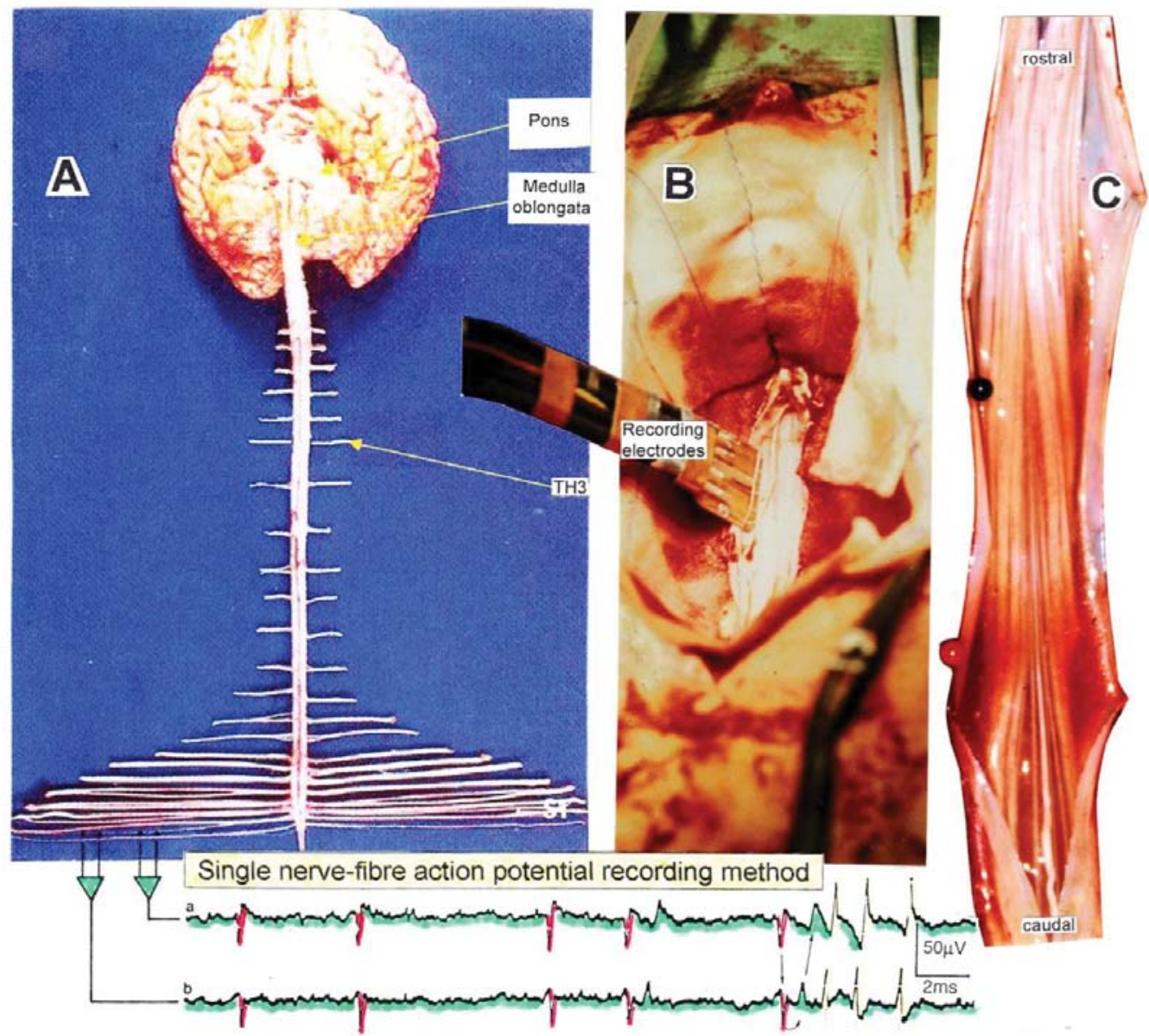

Figure 6: Layout of the recording of single-nerve fiber action potentials to measure the self-organization of neuronal networks of the human CNS under physiologic and pathophysiologic conditions. By recording with two pairs of wire electrodes (B) from sacral nerve roots (cauda equina, C), containing between 200 and 500 myelinated nerve fibers, records were obtained in which single-nerve fiber action potentials (APs) were identified from motoneuron axons (main action potential (AP) phase downwards) and afferents (main AP phase upwards). 


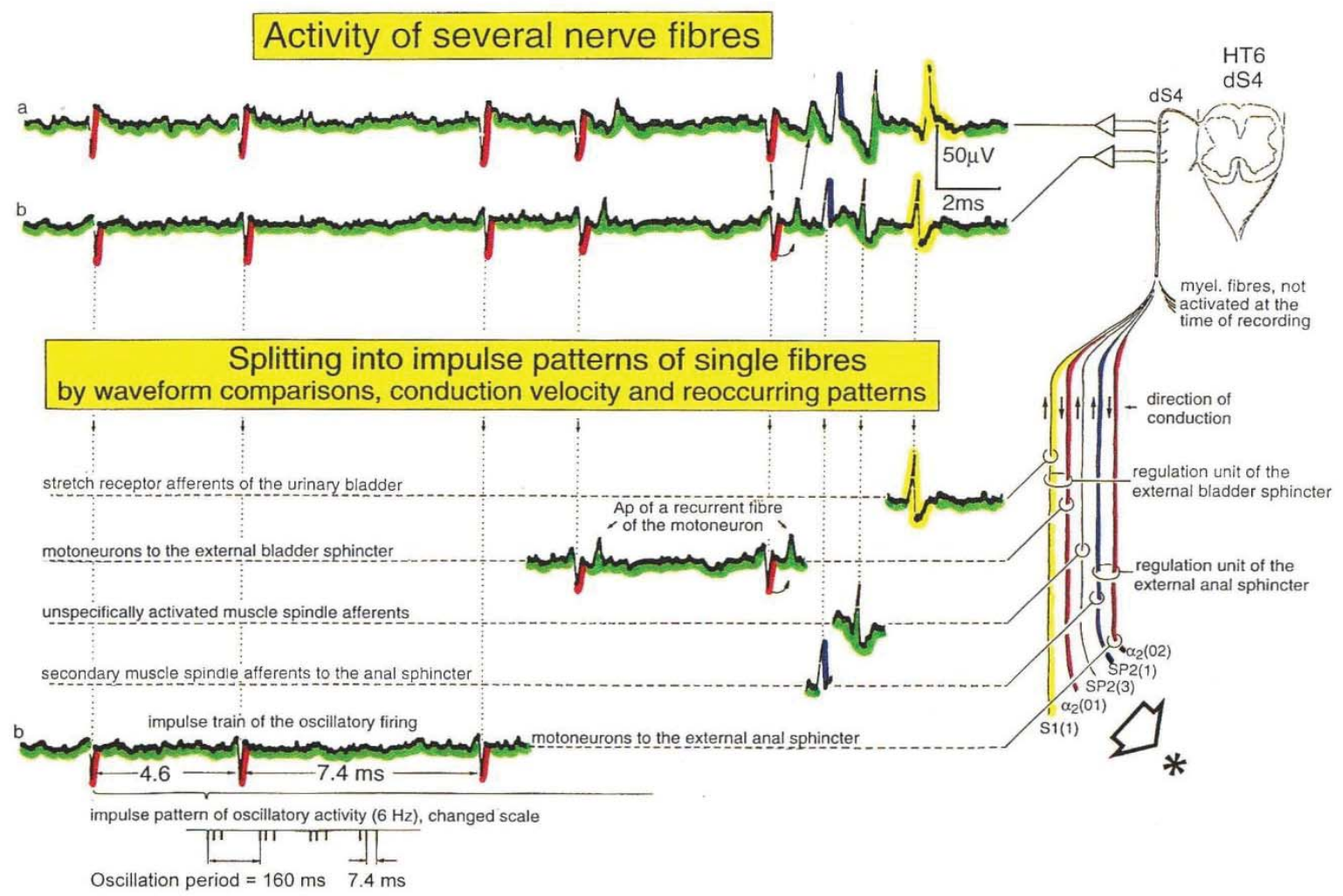

Figure 7: Schematic splitting of the activity of several nerve fibres into simultaneous impulse patterns of single fibres by comparing waveforms, conduction velocities and reoccurring characteristic impulse patterns (rhythmic firing of sphincteric motoneurons). The different conduction times and waveforms were recognized on an expanded time scale. Stretch receptor and secondary muscle spindle afferents contribute to the drive of sphincteric motoneurons and form, together with other afferents, regulation units.

But to identify from what nerve fibers the action potentials originate, a classification scheme for human nerve fibers is needed.

By measuring the conduction times and with the known electrode pair distance of $10 \mathrm{~mm}$, conduction velocity could be calculated and distribution histograms constructed in which the myelinated nerve fiber groups larger than $4 \mathrm{~mm}$ could be characterized by group conduction velocity values. After the recording, the roots were removed, fixated, stained and morphometry was performed. Distributions of nerve fiber diameters for different myelin sheet thicknesses were constructed and nerve fiber diameter groups were characterized by the peak values of asymmetrical distributions. By correlating the peak values of the velocity distributions with those of the diameter distributions, obtained for the same root, a classification scheme was constructed for the human peripheral nervous system (Figure 8) [16]. A group of nerve fibers is thus classified by a group conduction velocity and a group nerve fiber diameter. This classification scheme (Figure 9) is the only existing one for the human nervous system. With this classification scheme it became possible to record natural impulse patterns simultaneously from identified single afferent and efferent nerve fibers and analyze self-organizing mechanisms of the human CNS under physiologic and pathologic conditions [17-19]. Since conduction velocities depend on the temperature, a calibration relation is needed. Such a calibration relation is for the lower sacral nerve roots that the secondary muscle spindles afferent fibers conduct with the same velocity as the $\alpha_{2}$-motoneuron axons for different temperatures, marked in Figure 9 with red. For the classification of human nerve fibers, it is further important that the nerve fibers taper only very little. As measured, if there is no splitting or branching of fibers, the tapering is $0.2 \%$ per $13 \mathrm{~cm}[20]$. A $100 \mathrm{~cm}$ long fiber, for example of the pyramidal tract, would only reduce the diameter from $10 \mu \mathrm{m}$ to $9.8 \mu \mathrm{m}$. 


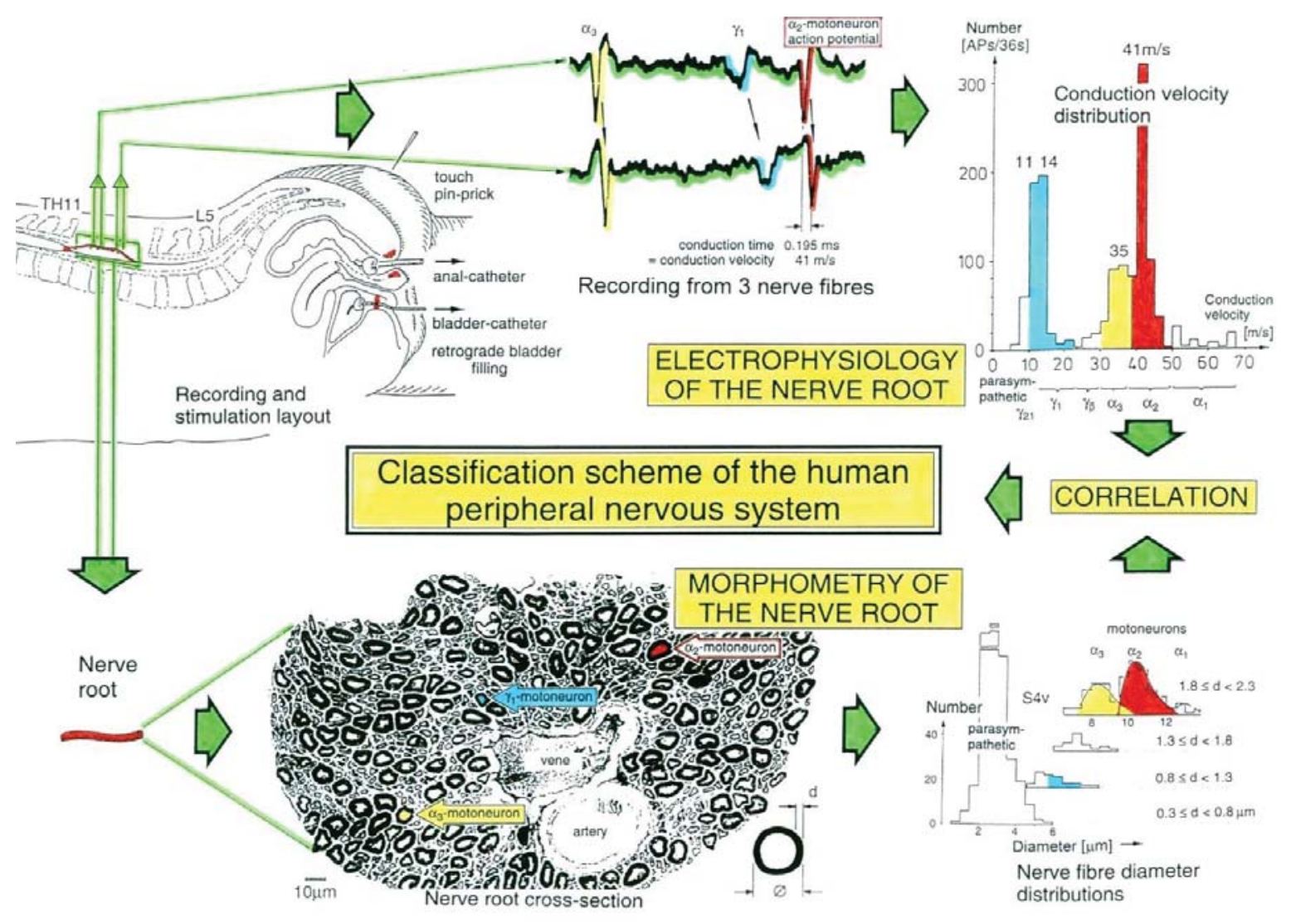

Figure 8: Development of a classification scheme for human peripheral nerve fibers. Conduction velocities (V) and nerve fiber diameters ( $\varnothing$ ) of afferent and efferent nerve fiber groups in normal humans and in patients with a traumatic SCI for 0.5 to 6 years.

1.8. Electrostimulation cannot simulate natural impulse patterns, is unphysiological and may damage neural network functions and learning transfer from movements to urinary bladder and sexual functions

Electrostimulation is unphysiological because the applied patterns cannot simulate the natural impulse patterns conducted in the different nerve fibers. Figure 10 shows the impulse patterns evoked by touch or pin-prick conducted in up to 22 skin afferent fibers. An electrostimulation of the skin cannot simulate such naturally generated impulse patterns caused by touch or pin-prick. Such impulse patterns inform the CNS about the outside world, which means in this case about touch and pin-prick. The by the electrostimulation generated patterns the CNS cannot understand.

The electro-stimulation of motoneurons or motor units is also unphysiological. Firstly, as Figure 11 shows, the three different kinds of motoneurons, innervating the three kinds of muscle fibers (FF, FR, S), have different activation frequencies and patterns. An electrostimulation can only serve a certain muscle fiber type at the surface of the muscle. Secondly, the motoneurons and the motor units do not fire physiologically in a synchronized manner to avoid tremor. They fire coordinately distributed to avoid synchronization of motor units and therefore tremor (Figure 12). The electrostimulation of muscles may enhance tremor in patients with Parkinson's disease.

In patients with an incomplete SCI there is some tendency for a synchronization of motoneurons because of loss of inhibitory tract fibers. Tremor can normally not be seen. But clonus is often observed in patients with SCI. In clonus, the feet perform consecutive dorsal and plantar flexions. The reason for that rhythmic dorsal and plantar flexion is probably a rhythmic activation of the very sensitive regulation loop between 

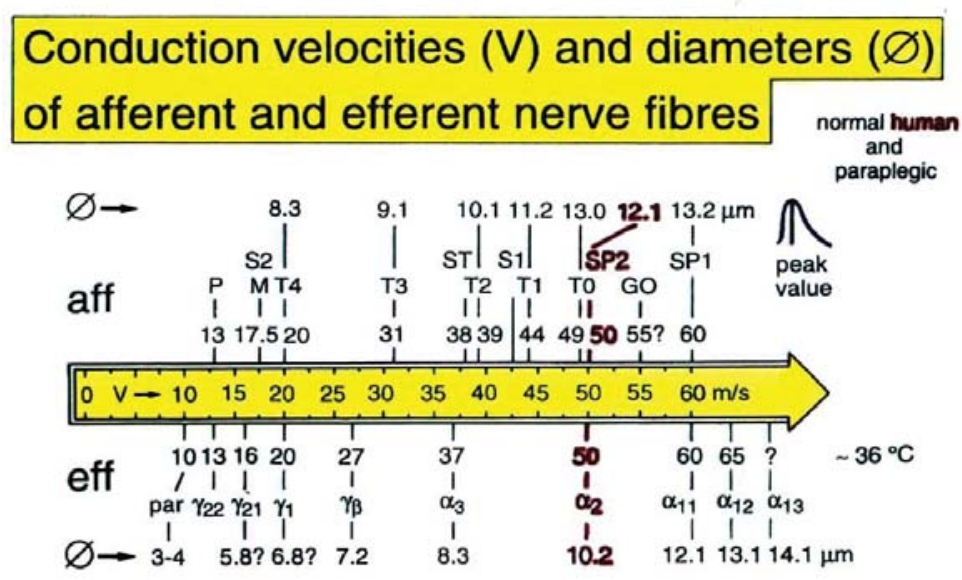

\begin{tabular}{|c|c|}
\hline $\begin{array}{l}\text { G0 } \\
\text { SP1 } \\
\text { SP2 }\end{array}$ & $\begin{array}{l}\text { Golgi tendon organ afferents } \\
\text { spindle afferents }\end{array}$ \\
\hline TO & touch-stimulated afferents \\
\hline PC T1 & \\
\hline T2 & \\
\hline $\begin{array}{r}\text { SAI T3 } \\
\text { T4 }\end{array}$ & 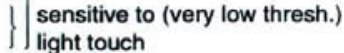 \\
\hline $\mathbf{P}$ & $\begin{array}{l}\text { fast conducting pain afferents } \\
\text { stimulated by pin-prick }\end{array}$ \\
\hline M & $\begin{array}{l}\text { mucosa afferents from } \\
\text { mechanoreceptors of bladder } \\
\text { and anal canal }\end{array}$ \\
\hline S2 & $\begin{array}{l}\text { bladder afferents responding } \\
\text { to fluid movement and filling of } \\
\text { more than } 600 \mathrm{ml}\end{array}$ \\
\hline $\begin{array}{l}\text { S1 } \\
\text { ST }\end{array}$ & $\begin{array}{l}\text { urinary bladder stretch and } \\
\text { tension receptor afferents }\end{array}$ \\
\hline
\end{tabular}

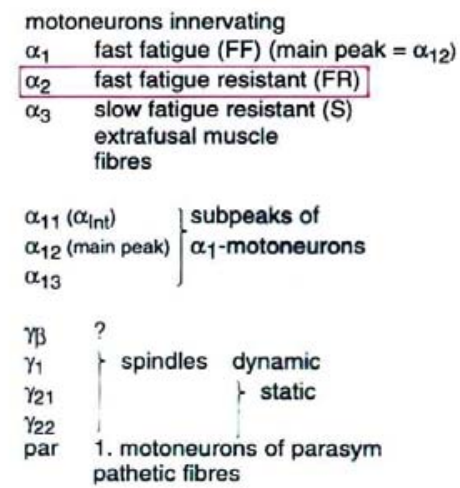

Figure 9: Classification scheme for human peripheral nerve fibers. Conduction velocities (V) and nerve fiber diameters ( $\varnothing$ ) of afferent and efferent nerve fiber groups in normal humans and in patients with a traumatic spinal cord lesion for 0.5 to 6 years. The splitting of the $\alpha_{1}$-motoneurons into the 3 subgroups, $\alpha_{11}, \alpha_{12}, \alpha_{13}$, has not yet been confirmed. This is the only existing classification scheme for human nerve fibers!

the feet and the spinal cord rather than synchronization of oscillatory firing motor units as in tremor (Figure 13).

Figure 13 shows EMG recordings of a patient with an incomplete SCI, having clonus and a pathologic motor program. The motor program is poor, because there is no motor program in the right tibialis anterior muscle and the motor bursts in the right and left gastrocnemius muscle are structured with rhythmicity, i.e. with clonus activity. A typical clonus recording when no exercising can be seen in Figure 13A. On a stretched time scale the clonus can clearly be seen in the right and left gastrocnemius muscles of Figure 13C. In the left gastrocnemius muscle the motor program burst is structured with three clonus bursts, which are in turn structured by rhythmic activity at 26 and $40 \mathrm{~Hz}$ which may originate from the oscillatory firing of FF-type motor units innervated by $\alpha 1$-motoneurons.

The unphysiological electrostimulation, applied to SCI patients, may increase the clonus and the synchronization of motor units (tremor) and ruin in this way the functional reorganization of neural networks and inhibit physiologic repair. The repair of urinary bladder and sexual functions by learning transfer from movements may become impaired. 


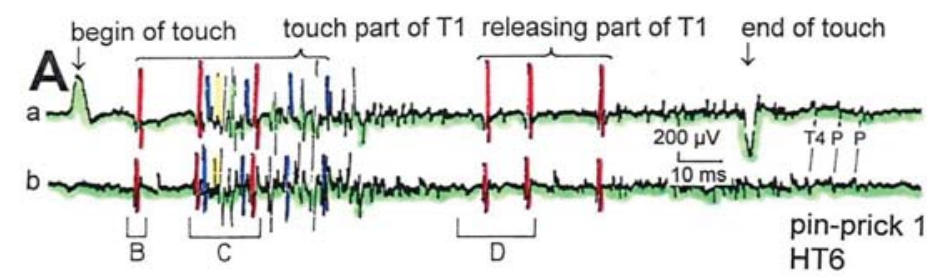

Touch (and pain) - stimulated afferent activity
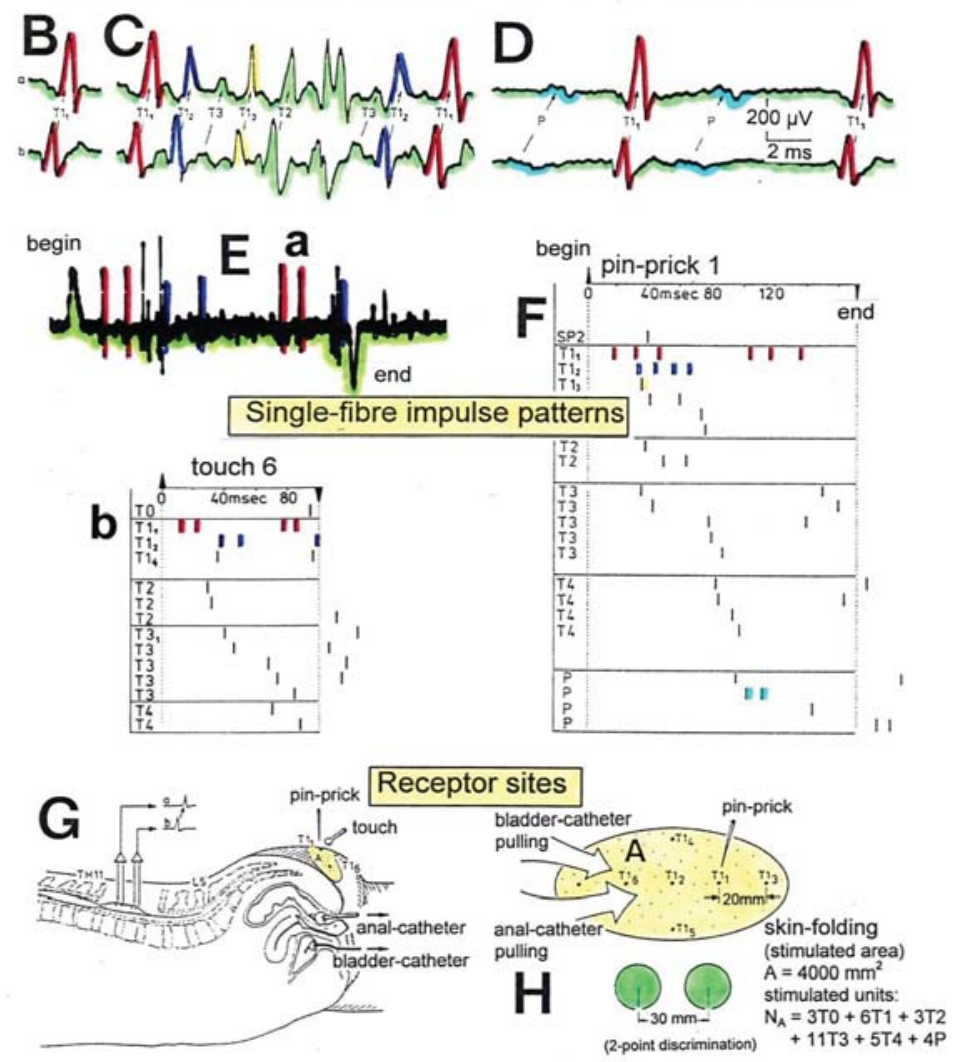

Figure 10: Touch (and pain)-stimulated afferent activity. Touch and pain activity, stimulated by pin-prick (A) and touch (Ea) of S5 or Co dermatomes and recorded extracellularly from a dorsal coccygeal root (brain-dead human HT6). T1, T2, T3, T4, P = mark action potentials (APs) from single touch and pain fibers. Subscripts 1, 2, 3 mark single fibers.

A. Whole sweep following pin-prick 1 shown at a slow time base. The large upward artifact on trace 'a' marks electronically the beginning of the pin-prick. The large downward artifact on trace 'a' marks the end of the pin-prick. Note that 2 intervals of high activity of large APs occur, one after the beginning of the pin-prick with $1 \mathrm{AP}$ in front, and a second before the end of the pin-prick; potentials with large amplitude are followed by potentials of small amplitude. Time intervals B, C and D are shown in a timeexpanded form in B, C and D.

B, C, D. Time expanded sweep pieces of A. Identified APs are indicated. Note that the APs from the $\mathrm{T} 1_{1}$ touch unit can be safely identified by the waveforms in B, C, D.

Eb, F. AP occurrence patterns of single touch and pain fibers following short touch 6 and pin-prick 1 . No pain afferents are stimulated upon touch 6. Upon pin-prick 1, the single-fiber AP activity of the different touch and pain groups is identified by the AP waveforms on traces ' $a$ ' and ' $b$ ', and by the conduction times. The single touch afferents of the T1 group are marked with subscripts. One active secondary muscle spindle afferent fiber (SP2) could always be identified in F. Note that for pin-prick 1, touch and pain afferents are stimulated whereas for touch 6 only touch afferents.

G. Recording and stimulation arrangement for simultaneous recording of several single touch and pain units. $\mathrm{A}=$ area stimulated by skin folding, drawn in $\mathrm{H}$ in more detail. $\mathrm{T} 1_{1}, \mathrm{~T} 1_{6}=$ suggested touch points of the $\mathrm{T} 1_{1}$ and $\mathrm{T} 1_{6}$-units.

H. Drawing of the very approximate skin area stimulated by skin folding. $\mathrm{T} 1_{1-6}=$ suggested focal $\mathrm{T} 1$ touch points. Two-point discrimination indicated for the sake of comparison. $\mathrm{N}_{\mathrm{A}}=$ number of stimulated units in the dorsal coccygeal root. Skin tractions evoked by anal and bladder-catheter pulling are indicated by the large open arrows. 


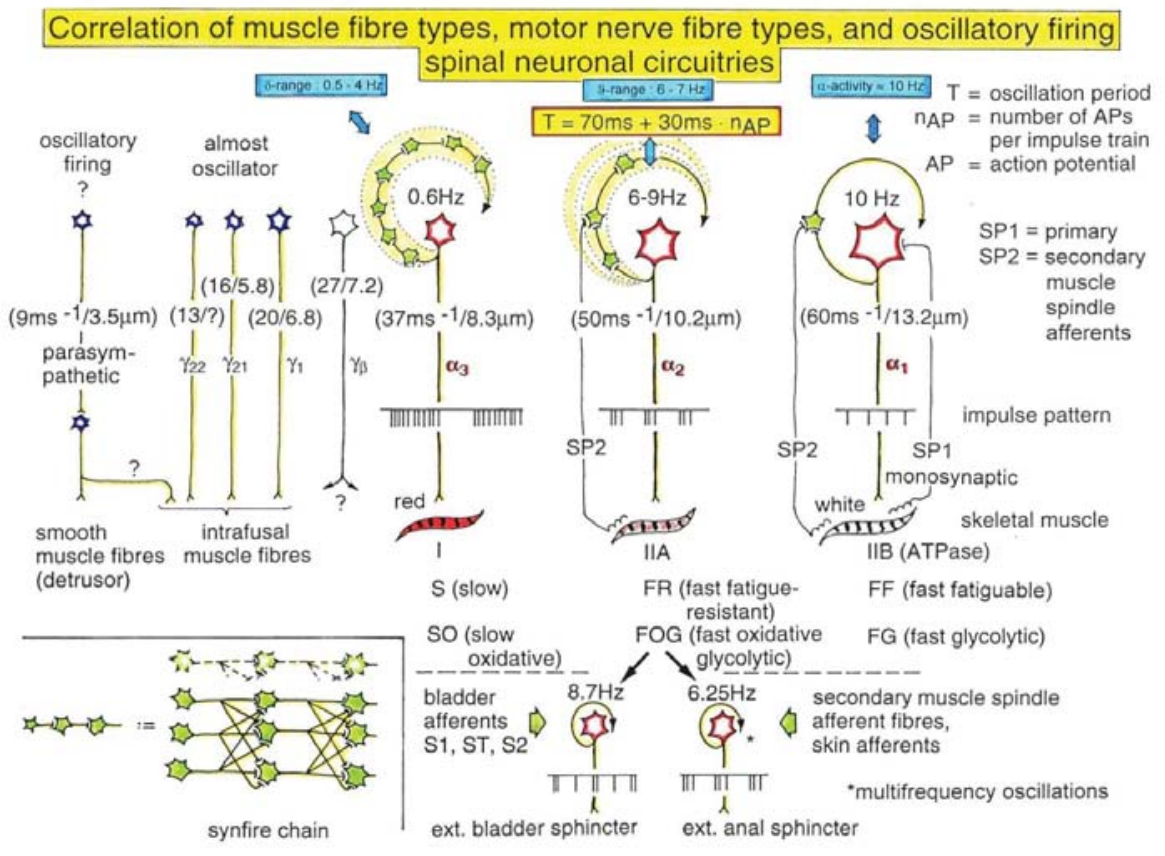

Figure 11: Correlation of muscle fiber types, motor nerve fiber types, and oscillatory firing spinal neuronal networks, based on histochemical, morphological and physiological properties. This figure provides a simplified correlation between muscle fiber, motoneuron and sacral oscillator types. No additional subtypes have been included. $\alpha=$ motoneuron, $\gamma_{1}, \gamma_{2}=$ dynamic and static fusimotors, parasympathetic = parasympathetic preganglionic motoneuron. S1, ST, S2 = stretch, tension and flow receptor afferents.

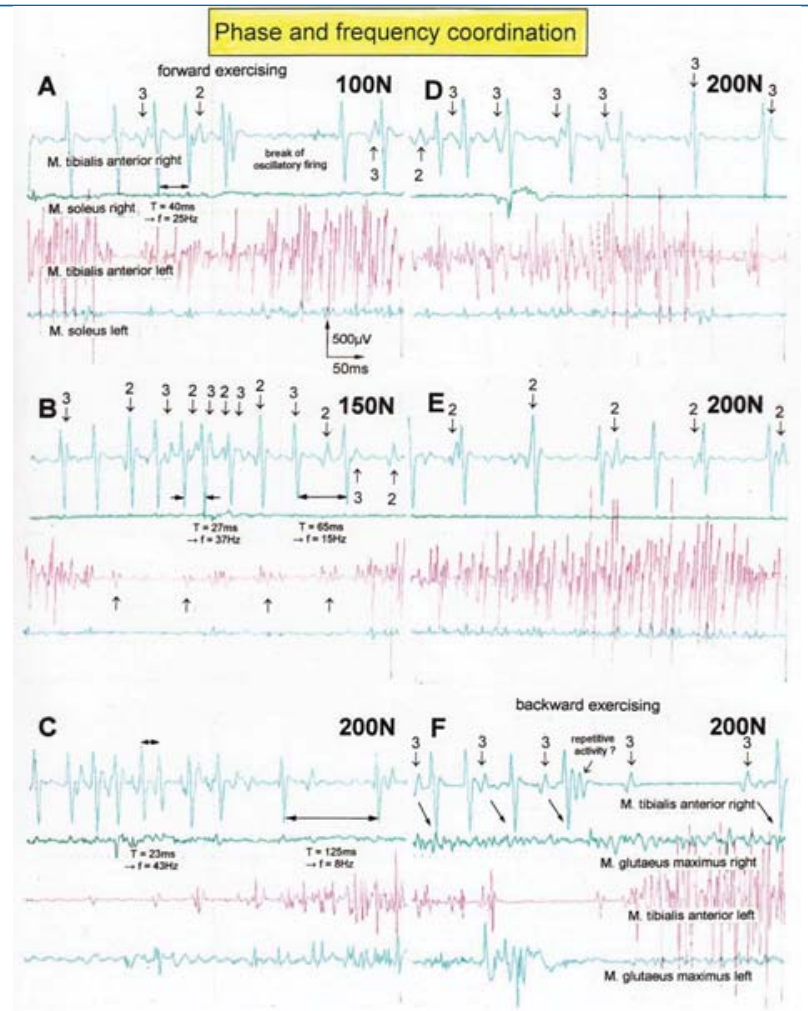

Figure 12: Phase and frequency coordination between oscillatory firing motor units (FF-type) during the generation of a motor program during exercise on the special CDT device at loads increasing from 100 to $200 \mathrm{~N}$. Oscillation periods (T) and oscillation frequencies (f [Hz]) of oscillatory firing motor unit 1 are partly indicated. 'A,B,D,E' same recording situation as in Figure 23; 'C,F' soleus electrodes shifted to gluteus muscles to check early re-innervation upon therapy. The waveforms of the 3 identified FF-type motor unit potentials ' 1 ', ' 2 ', and ' 3 ' are the same as in Figure 23 ; motor units ' 2 ' and ' 3 ' are partly marked. In ' $F$ ', some coordination's between motor unit ' 3 ' and ' 1 ' are marked. 

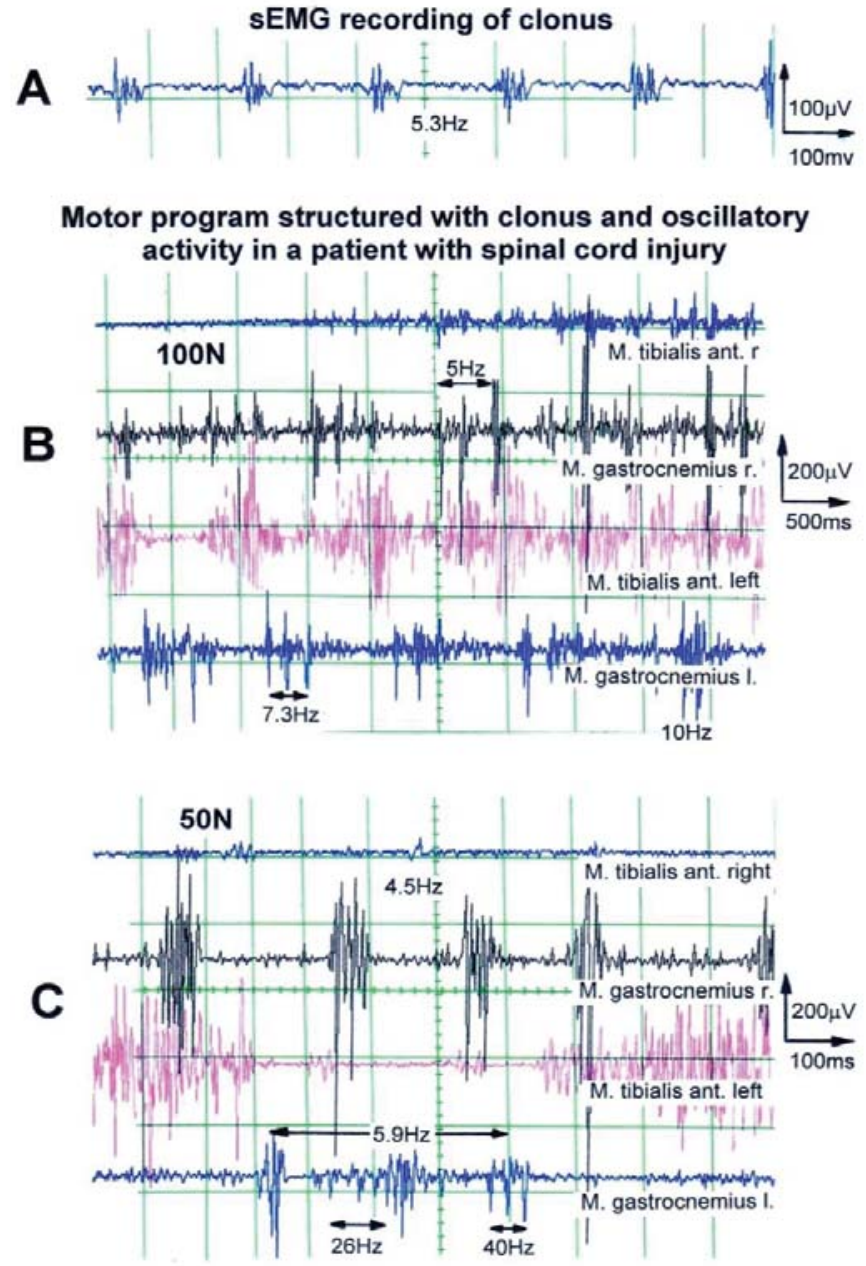

Figure 13: Motor program bursts in patients who suffered a SCI, structured with clonus activation and rhythmic firing of FF-type motor units A. EMG recording of a clonus $(\mathrm{f}=5.3 \mathrm{~Hz})$ in the right tibialis anterior muscle of a patient who suffered a complete SCI sub Th5/7; the patient was not exercising. B, C. Motor programs of a patient who suffered an incomplete SCI sub Th4 upon exercising on the special CDT device at 50N and 100N (medium to high load). In B, motor program bursts are structured by rhythmicity; frequencies of 5 and $7.3 \mathrm{~Hz}$ are suggested. No motor program in the right tibialis anterior muscle; some motor program structure in the right gastrocnemius muscle. In C (faster sweep), there is no motor program in the right tibialis anterior muscle. Mainly clonus activity at a frequency of $4.5 \mathrm{~Hz}$ can be seen in the right gastrocnemius muscle. Two physiologic motor program bursts can be seen in the left tibialis anterior muscle (not structured by rhythmicity). In the left gastrocnemius muscle a motor program burst can be seen which is structured by $5 \mathrm{~Hz}$ rhythmicity (clonus frequency, see clonus in the right gastrocnemius muscle) and higher frequency rhythmicity (26 and $40 \mathrm{~Hz})$. 


\section{Method}

\subsection{Coordination dynamics therapy (CDT)}

CDT is a movement-based learning therapy to repair the human central nervous system (CNS) to regain lost functions back. For a recent review see [3]. In short, following brain, brain stem or SCI, the self-organization of neural networks by phase and frequency coordination becomes impaired besides the loss of functions due to damage or loss of nervous tissue. Through CDT this impaired phase and frequency coordination has to be improved through exercising on a special CDT device (Figure 16) and other movements. Some lost functions re-appear already then. To regain lost functions, movements have to be trained to repair impaired functions and other brain and spinal cord parts have to take function over by plasticity. The trained movements include creeping, crawling, up-righting, walking, running and jumping. Only those movements can be administered to the patient which the patient can perform with or without support. The main CNS repair is achieved through a functional re-organization. The capacity for a regeneration of the spinal cord and the building of new nerve cells is very limited in human. In this report it is tried to increase the capacity of regeneration and neurogenesis and to understand its induction. It is the first time in history that a regeneration of the human spinal cord could be achieved, based on human neurophysiology and clinics and documented by diagnostic.

\subsection{System Theory of Pattern Formation for understanding neuronal network organization and learning and measure repair progress}

To apply successfully CDT, the System Theory of Pattern Formation is needed to understand better the repair and measure the improvement of CNS organization. In a complex system like the human CNS, patterns are generated by the nervous system which seeks cooperative stability. Stability is what defines collective states. The system has the tendency to slip into the collective states to which it is attracted. When an infant crawls (Figure 14), its arms and legs are strongly attracted to the 'pace' and 'trot' gait coordination patterns. The attraction is so strong that intermediate crawling patterns seemingly do not exist, as if the patterns are hard-wired. But with the help of the special CDT device, the CNS can generate intermediate coordination patterns. A patient with a CNS injury often crawls with intermediate arm and leg coordination patterns and has to re-learn the pace and trot gait coordination's for CNS repair and shifts in this way the attractors for crawling to the pace and trot gait coordination's. Attractive states and attractors of CNS organization can be pictured as a ball in a potential well or more generally in an attractor layout (Figure 15). Changes in

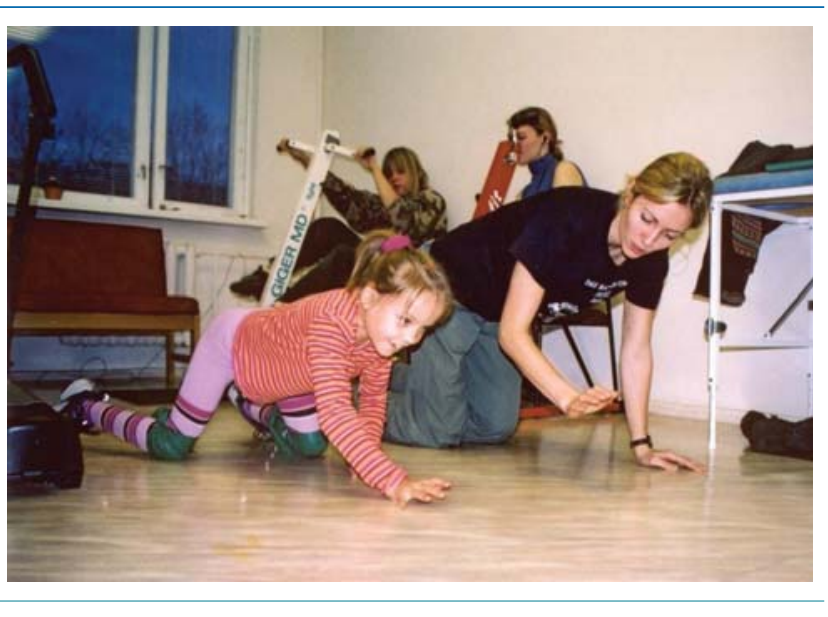

Figure 14: Trot gate crawling of a cerebral palsy girl in interpersonal coordination with the therapist. The crawling performance of the therapist is not optimal. The right arm is leading with respect to the left knee. The crawling performance of the patient is also not optimal; the knees are too much apart. 
CNS functioning are characterized as continuous stabilization and destabilization, over time, of preferred attractor states.

To reduce for understanding the complexity of human neural networks of the many billions of neurons, order parameters or collective variables are introduced for the generation of certain movements. An equation of motion describes the coordination patterns dynamics. However, coordination patterns are not only determined by the task or biological function. Patterns adjust continuously to requirements from the environment (transmitted by impulse patterns from stimulated receptors in the periphery), memory, intention, and support given by a therapist. The specific requirements are captured by the concept of behavioral information and are made part of a vector field that attracts toward the required patterns. The coordination pattern dynamics, characterized by equations of motion of collective variables (the vector $\mathrm{X}$ ), takes the general following form [21].

$$
\frac{d X}{d t}=F_{\text {intr }}(X)+\sum c_{\text {inf }} F_{\text {inf }}(X, t)
$$

where $F_{\text {intr }}$ designates the intrinsic dynamics of the nervous system. These intrinsic dynamics capture the anatomical (neuronal network structure), physiological and pathological states of the CNS and its muscularskeletal elements. $\sum c_{i n f} F_{i n f}(X, t)$ represents the sum of external influences $\left(F_{\text {inf }}(X, t)\right)$ with their relative strength $\left(c_{i n f}\right)$ pertaining to each influence. The so-called behavioral information $F_{\text {inf }}(X, t)$ includes cognitive states, emotional states, intentions, motivations, instructions, inter-personal coordination, movement support etc. During motor learning or while applying therapy to a patient these extrinsic influences become extremely important, because the intrinsic (pattern) dynamics can be changed with these extrinsic influences by altering the equation of motion. By modulating the behavioral information, the intrinsic dynamics of the neuronal networks can be influenced further, that is if CDT is no longer efficient in repairing the injured CNS, requiring the therapy to be updated. With respect to a healthy athlete, the movement performance can be improved by modulating the behavioral information by for example including in the training program the exercising on a special CDT device to improve CNS functioning.

If the behavioral information includes the exercising of extremely coordinated, integrative movements, like exercising on the special CDT device, then the quality of CNS self-organization can be enhanced by improving the exactness of self-organization, namely the precision of phase and frequency coordination between neuron and neural assembly firings. By improving the precision of organization of the intrinsic dynamics, that is the specific variability of the injured networks, certain patterns do eventually re-appear in the case of repairing the injured CNS by movement-based learning.

\subsection{Learning implications for treatment derived from the equations of motion of the collective variables}

From the repair by learning in the severely injured CNS we learn about learning in the healthy CNS, because the impact on the learning mechanisms is higher than in animal experimentation.

1. Behavioral requirements Finf (like intention, support, and instruction) affect the whole coordination dynamics, including stability, rather than only certain coordination patterns. The change of the whole coordination pattern dynamics of the CNS by the behavioral information is one scientific basis for learning transfer [22] between different patterns and stability changes of patterns (as for example the reduction of spasticity). The other scientific basis for learning transfer is followed from human neurophysiology, namely that nerve cells or neural sub-networks are involved in different neural network organizations [23].

2. The intrinsic dynamics Fintr include vegetative and higher mental functions (these are also patterns of the coordination dynamics), which indicate that via exercising coordinated movements with support and/or instructions $\left(F_{i n f}\right)$, urinary bladder function, intelligence and speech can be partly repaired or improved following CNS injury or malformation. 
3. When in an injured CNS with a certain set of behavioral information $\left(\sum c_{\text {inf }} F_{\text {inf }}\right)$ the intrinsic coordination dynamics $\left(F_{\text {intr }}\right)$ can no longer be influenced during CDT, then this set of behavioral information has to be changed (using different $F_{\text {inf }}$ ), or balanced differently (using different cinf), to further improve CNS organization dynamics.

4. However, the equations of motion of the coordination pattern dynamics (formula 2) provide no information about the specific behavioral information $\left(F_{i n f}\right)$ and training intensity $\left(c_{i n f}\right)$ with which the CNS can be efficiently repaired by learning in a patient. We need to have detailed knowledge of the human CNS at the single neuron and neural assembly level, as well as knowledge at the integrative level, to find the specific behavioral information for the repair by learning of the human CNS.

A first novel step in CDT is the inference derived from the equation of motion. It suggests that the movement learning not only improves the performance of that particular movement, but also improves the other non-trainable functions by transfer of learning $[22,23]$. These functions include vegetative functions like bladder control, speech and higher mental functions.

Furthermore, we have means by which the stability of physiological network states can be increased (e.g. movements, continence, continuous concentration in performing certain tasks, speech etc.) and simultaneously the stability of pathological network states, like spasticity, decreased. The coordination (pattern) dynamics therapy partly based on the System Theory of Pattern Formation in combination with human neurophysiology thus offers us an important theoretical basis and a practical tool to diagnose, quantify and repair/improve the functioning human nervous system at the macroscopic level.

\subsection{Geographical landscape of attractors}

The drawback of the equation of motion of the order parameters (formula 2) is that it is normally not possible to find a mathematical solution to it. But by defining a potential function and by picturing the attractive states and attractors by a ball in a potential well or rather by a ball moving in a geographical landscape of attractors (Figure 15), we form a theoretical basis to understand and measure stability of certain coordinated movement patterns (i.e. the deepness of the potential well of an attractor) in patients with CNS injury who receive on-going therapy.

To make the strategy of pattern formation, pattern stability, pattern assessment, and pattern picturing understandable, the procedure is demonstrated for the simple movement 'jumping on springboard' (Figure 15D), which is used during CDT, especially for the repair of the urinary bladder and training in the up-right weight-bearing posture (very important in patients with SCI).

For the special movement 'jumping on springboard' with no behavioral information $\left(\sum c_{i n f} F_{i n f}(X, t)=0\right)$ the equations of motion (formula 2) take the form $\frac{d \varphi}{d t}=f_{\text {intr }}(\varphi)$, where $\varphi$ is the relative phase between the two moving legs and is the only collective variable of this special movement.

The mathematical solution of $\frac{d \varphi}{d t}=f_{\text {intr }}(\varphi)$ in the Haken-Kelso-Bunz model $[24,25]$ gives the equation of motion for jumping on a springboard for the symmetric case. The obtained potential function $V(\varphi, t)=-a(t) \cos \varphi-b(t) \cos 2 \varphi$ can be plotted for different $\varphi$ and certain ratios of the parameters a and $\mathrm{b}$ and is shown in Figure 15.

The potential function shows two attractor states, namely the jumping in in-phase $(\varphi=0)$ and the jumping in anti-phase $(\varphi= \pm \pi)$. Especially for higher frequencies (smaller b/a) the jumping in-phase has a higher stability (the potential well is deeper) than the jumping in anti-phase. Asymmetry (not tackled mathematically here) strongly changes the stabilities of the attractor states (depths of potential wells) (Figure 15). 
The human CNS, seeking for cooperative stability, slips into the collective states to which it is attracted. For jumping on springboard these attractive states are the jumping in in-phase and in anti-phase. For crawling (Figure 14) the attractive states are the pace (in-phase) and in trot gait coordination's (anti-phase). When introducing the variability of phase and frequency coordination of strength $Q$, the potential function takes the form $\frac{d \varphi}{d t}=\frac{d V(\varphi)}{d \varphi}+(Q \xi t)^{1 / 2}$, where $» t$ is Gaussian white noise of unit variance.

The behavioral changes when jumping on springboard (Figure 15) are represented by the overdamped movement of a rolling ball in the potential landscape for the physiologic (Figure 15A, Q small = little fluctuation of phase and frequency coordination) and the pathologic case (Figure 15B,C; Q large = large variability). The increased fluctuation in the rather stable state, due to increased variability of phase and frequency coordination, will have greater probability of "kicking" the system out of attractor the basin (Figure 15B,C), especially in the asymmetric case.

In the healthy CNS, the phase and frequency variability is small (short arrows) and the jumping inphase and anti-phase is stable (Figure 15A). Following injury, the potential landscape is deformed and the fluctuation of the network states, generating jumping, is high (Figure 15B). The in-phase jumping is still stable in spite of the increased fluctuation, because the basin of attraction is deep. The jumping anti-phase became unstable because the basin of attraction is shallow and the increased fluctuation in the state has a greater probability of "kicking" the system out of the basin. A switch into a spastic state is also possible. In severe CNS injury or malformation, the patient cannot jump any more in anti-phase because of the missing of attractors for anti-phase jumping (Figure 15C). Support is needed for anti-phase jumping. The jumping in-phase is still possible but unstable.

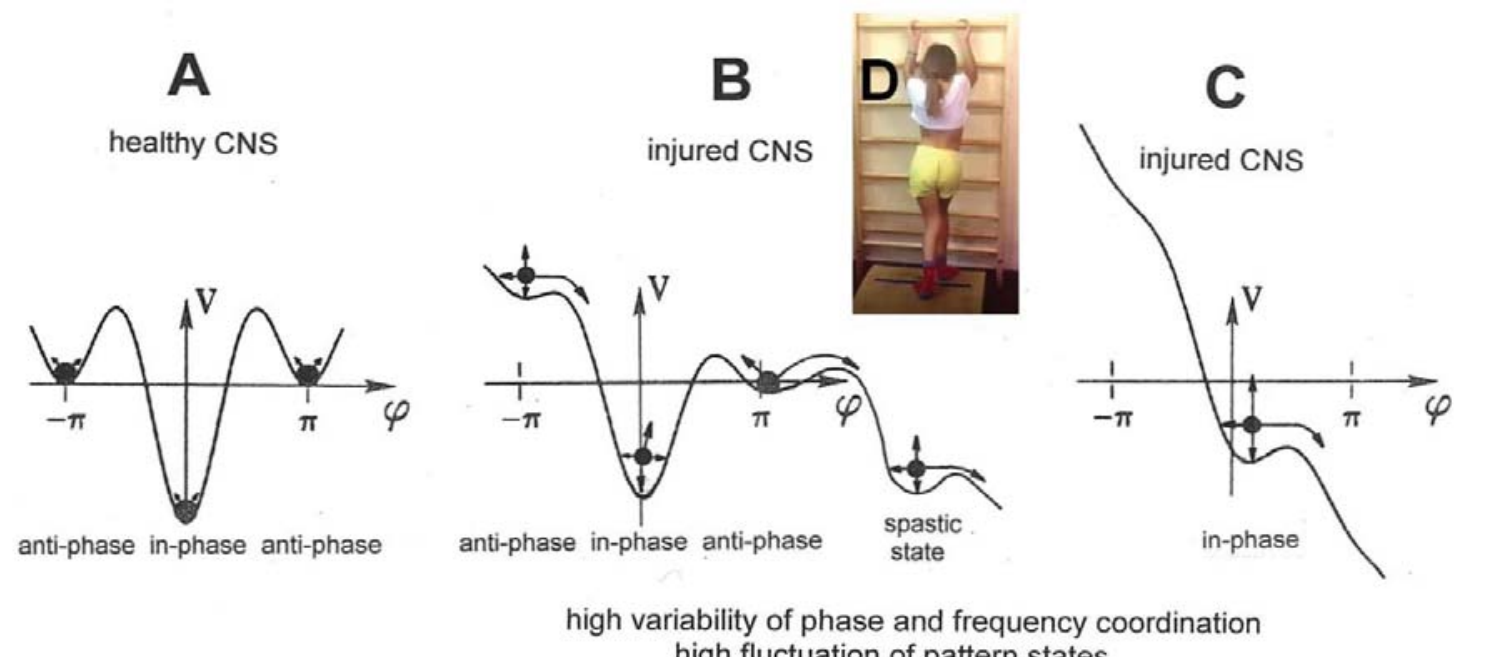

high fluctuation of pattern states

Figure 15: The potential, $\mathrm{V}(\varphi)$, of the coordination dynamics for jumping on springboard (D, Nefeli) of a healthy (A) and injured CNS $(B, C)$. The region around each local minimum acts like a well that weakly traps the system into a coordinated state. Behavioral changes are represented by the over-damped movement of a rolling ball in the potential "landscape". High fluctuations (indicated by long arrows attached to the ball (network state)) in the stable state, due to high variability of phase and frequency coordination (in the injured case), will have a greater probability of "kicking" the system out of the basins of attraction $(B, C)$ than for low fluctuations (short arrows) (A), due to small variability of phase and frequency coordination (in A). In B, only the in-phase jumping is stable, even though the fluctuation is high. In C there is only an attractor basin for the in-phase jumping, but the fluctuation is so high that there is a high probability that the system is kicked out of the basin of attraction. The patient can no longer jump in anti-phase and has difficulty with jumping in-phase. The stability of jumping depends on the motor program (deepness of basin of attraction) and the fluctuation of the pattern state (moving of the ball) caused by the increased variability of phase and frequency coordination due to the injury. 
Upon performing very exact coordinated movements, imposed by devices (Figure 16), the nervous system of the patient learns to reduce the variability of phase and frequency coordination and achieves in this way a small fluctuation of the network states again as shown in Figure 15A. The progress in treatment (learning) is that the in-phase jumping in Figure $15 \mathrm{C}$ and the anti-phase jumping in Figure 15B become stable again (Figure 15A). Also, the potential landscape will change due to the reduction of the phase and frequency variability. The important consequence for treatment is that when exercising on special CDT devices and reducing in this way the variability of phase and frequency coordination, the patient can induce the formation of patterns again, without

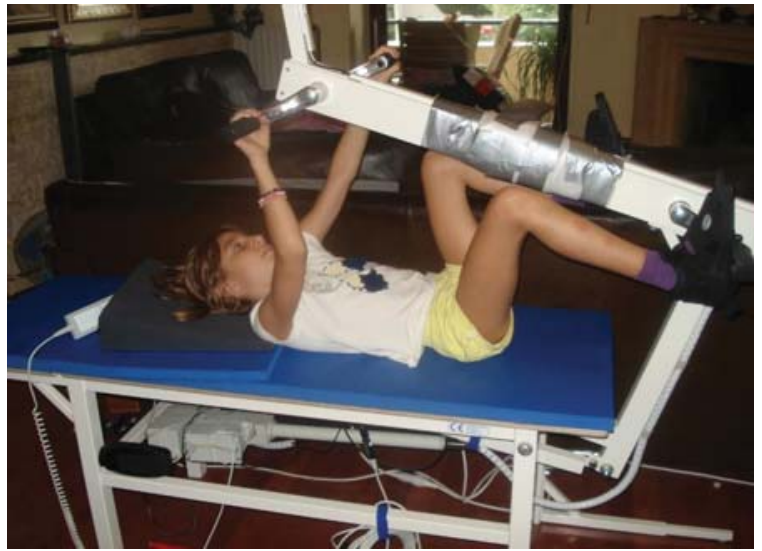

Figure 16: The 11-year-old Nefeli with an incomplete SCI during exercising coordinated arm, leg and trunk movements to improve the coordinated firing of neurons and sub-neural networks. This special CDT device for measuring and therapy (int.pat.) is produced by the firm: Giger Engineering, Martin Giger dipl. Ing. ETH/SIA, Herrenweg 1, 4500 Solothurn, Switzerland, www.g-medicals.ch. having trained them (learning transfer). Upon improving the coordinated firing of neurons, a cerebral palsy child my become able to speak or may develop social behaviors.

In conclusion, the impairment of phase and frequency coordination caused by CNS injury, measured at the single-neuron level in human, can be included in the coordination pattern dynamics at the collective variable level. The decrease of the variability of phase and frequency coordination (one kind of coordination repair) is an essential part of CNS development and repair by movement-based learning.

\subsection{Quantifying the improvement of CNS functioning by measuring pattern stability upon pattern change when exercising on the special CDT device}

Experimentally, the underlying dynamics of coordinated movements can be found in the temporal stability of coordination patterns and can be assessed through pattern change (second novel step). A change of the coordinated movement patterns is generated, when a subject is exercising on the special CDT and recording device (Figure 17), where the coordination between arms and legs, imposed by the device, changes continuously between pace $(\mathrm{P})$ and trot gait $(\mathrm{K})$ and backwards. The stability of the intrinsic coordination pattern tendencies is measured by the deviations and differential stability during the performance of these rhythmic movements. When the differential stability of the movement pattern is high, the arrhythmicity of exercising is small and when the stability is low the arrhythmicity of exercising in that pattern is high. From a standard coordination dynamics trace it can be seen that in the healthy case the arrhythmicity is low for the pace and trot gait coordination's and is high for the intermediate coordination's between pace and trot gate. The pace and trot gait coordination's between arm and leg movements have a high stability (low amplitude arrhythmia) and the intermediate coordination patterns have a low stability (high arrhythmia).

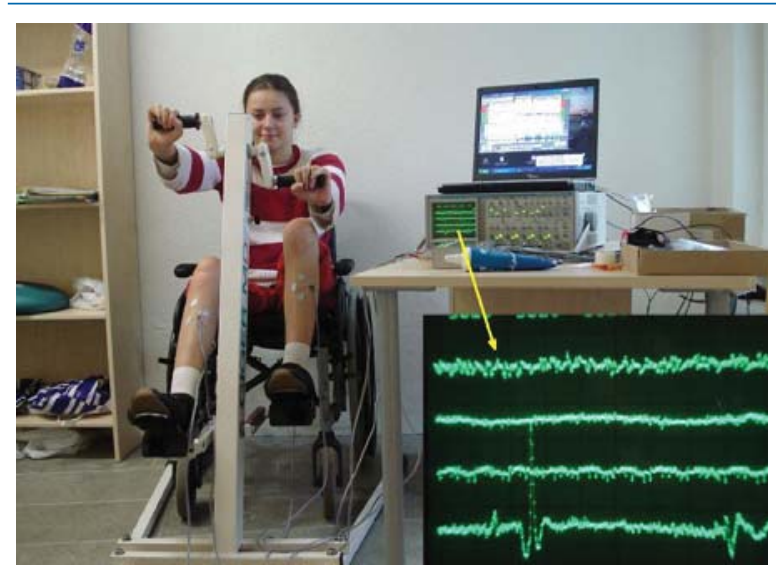

Figure 17: Layout for measuring coordination dynamics (arrhythmicity of exercising) between arm and leg movements, displayed on the laptop; for the intermediate coordination's between pace and trot gait, the fluctuation of the network states is larger. The recording of sEMG activity (displayed on the oscilloscope) from the tibialis anterior and other muscles is also shown. The inset shows single motor unit action potentials on the lowest trace. The recordings are taken from a patient (Kadri) with a motoric complete cervical SCI C5/6 (95\% injury). 


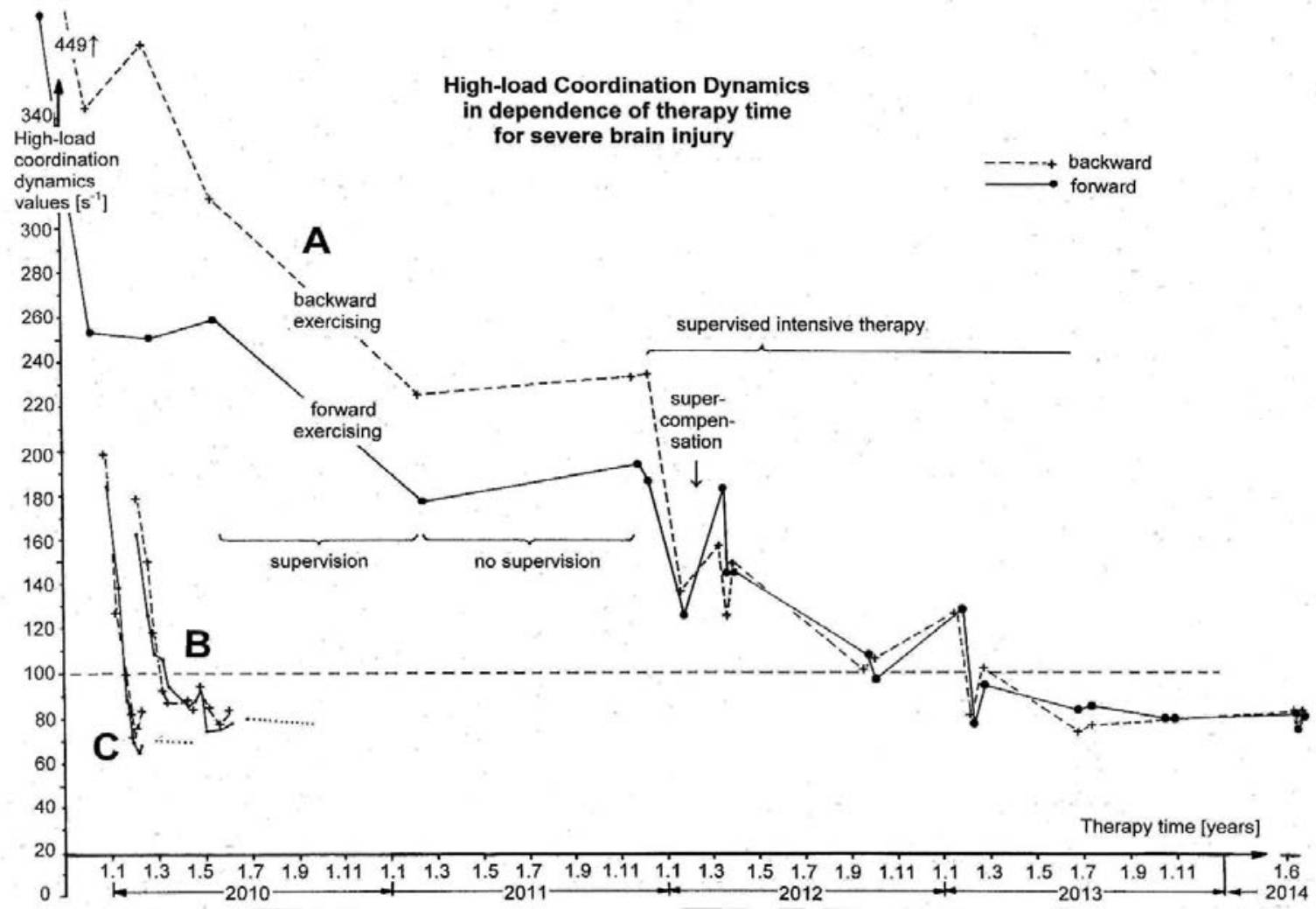

High-load coordination dynamics values $[1 / \mathrm{s}]$

High-load Coordination Dynamics in dependence of therapy time for severe brain injury

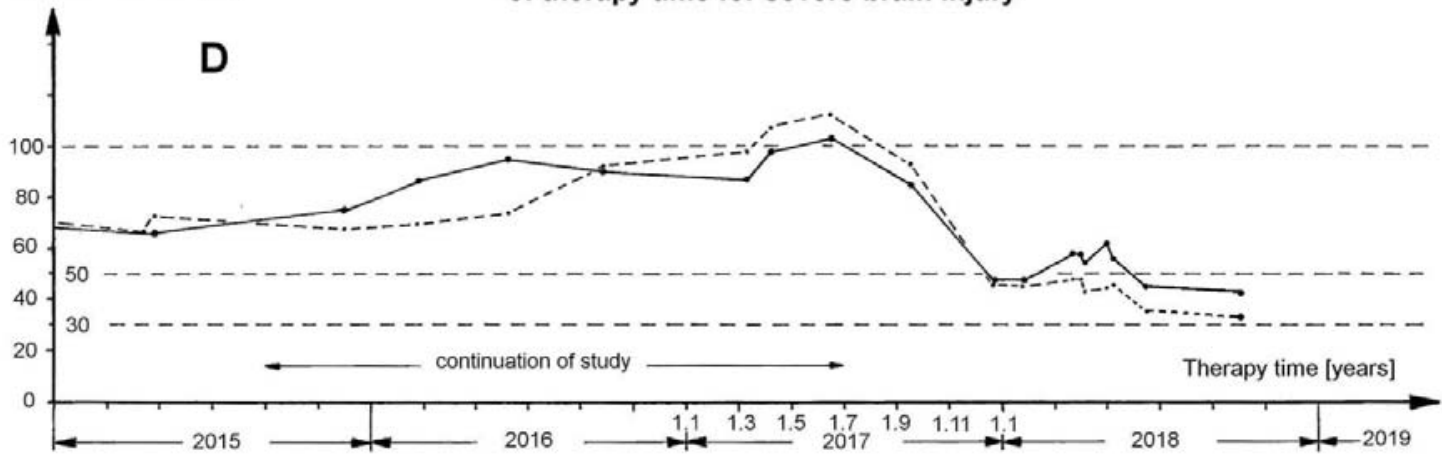

Figure 18: A. Improvement of high-load coordination dynamics (CD) values in a patient with severe brain injury upon CDT for several years. The high-load CD values were obtained by summing up the single CD values for forward and backward exercising, (high-load CD value) $=\Delta 20 \mathrm{~N}+\Delta 50 \mathrm{~N}+100 \mathrm{~N}+\Delta 150 \mathrm{~N}+200 \mathrm{~N}+\Delta 150 \mathrm{~N}+\Delta 100 \mathrm{~N}+100 \mathrm{~N}+\Delta 50 \mathrm{~N}+\Delta 20 \mathrm{~N}+\Delta 20 \mathrm{~N}$ ). B, C. For comparing the rate of repair, the improvement curves of the high-load coordination dynamics values of an athlete (C) and a healthy pupil (B) are inserted. Note that the brain-injured patient needed much more time to achieve similar good CD values. D. Continuation of therapy. The substantial improvement of the high-load CD values (lower values), in the middle of 2018, motivated the patient Sotiris to continue his therapy at the limit.

The mean stability per minute can be measured by the arrhythmia of exercising $\left(\frac{d f}{d t}: f, f=\right.$ frequency; ord $\frac{v}{d t}, v=$ angular velocity). Such differential stability value per minute, the so-called coordination dynamics (CD) value, quantifies CNS functioning objectively, integrative and non-invasively. The practical assessment of quality of CNS organization by pattern change is a third novel step in CDT. 
In Figure 18 it is shown for a patient with a very severe brain injury (Sotiris) following a car accident that brain repair, quantified by high-load coordination dynamics (CD) values, needs years. This patient needed 4 years to reach comparable good high-load CD values. The repair time in SCI depends strongly on the severance of the injury and is longer. For comparison, the rate of repair (the improvement curves of the high-load coordination dynamics values) of an athlete (C) and a healthy pupil (B) is inserted. Note that the brain-injured patient needed much more time to achieve similar good (low) CD values. The athlete and the pupil needed for the substantial improvement of brain functioning approximately 1 month. For roughly the same nervous system improvement the patient with the very severe brain injury needed 4 years, that means the efficiency of movement-based learning was lower by approximately a factor of 50 . This means, CNS repair through learning needs years. 


\section{Results}

\subsection{Review of spinal cord injury repair via coordination dynamics therapy}

3.1.1 Repair in a group of spinal cord injury patients through $\mathbf{3}$ months of CDT : CDT was applied to 18 patients (average age 31 years, after a SCI between C4/5 and L4/5; the therapy was administered on average 5 years after the injury for a minimum of 3 months. The injuries were due to car accidents, working accidents, jumping into the water, motor-cycle accidents, playing ice hockey, a parachute accident and erroneous surgery. All (almost) complete SCI became incomplete, i.e. motor functions improved below the injury level, including trunk stability and arm, hand and leg functions. The organization of the CNS, quantified by the CD values, improved by $42 \%$ for forward and by $49 \%$ for backward moving when exercising on a special CDT device. The improvements of the CD were 53\%, 32\% and 48\% for injuries in the cervical, thoracic, and lumbar range, respectively. Since the CD values did not change substantially prior to CDT, it did further improve with continued therapy, and worsened when the therapy was terminated, it is concluded that the improvement of CNS functioning above and below the SCI level was due to the therapy. As will be shown below SCI can partly be repaired if CDT is administered intensively with an efficient Therapy for a few years. With the improvement in single patients, details of repair will be given.

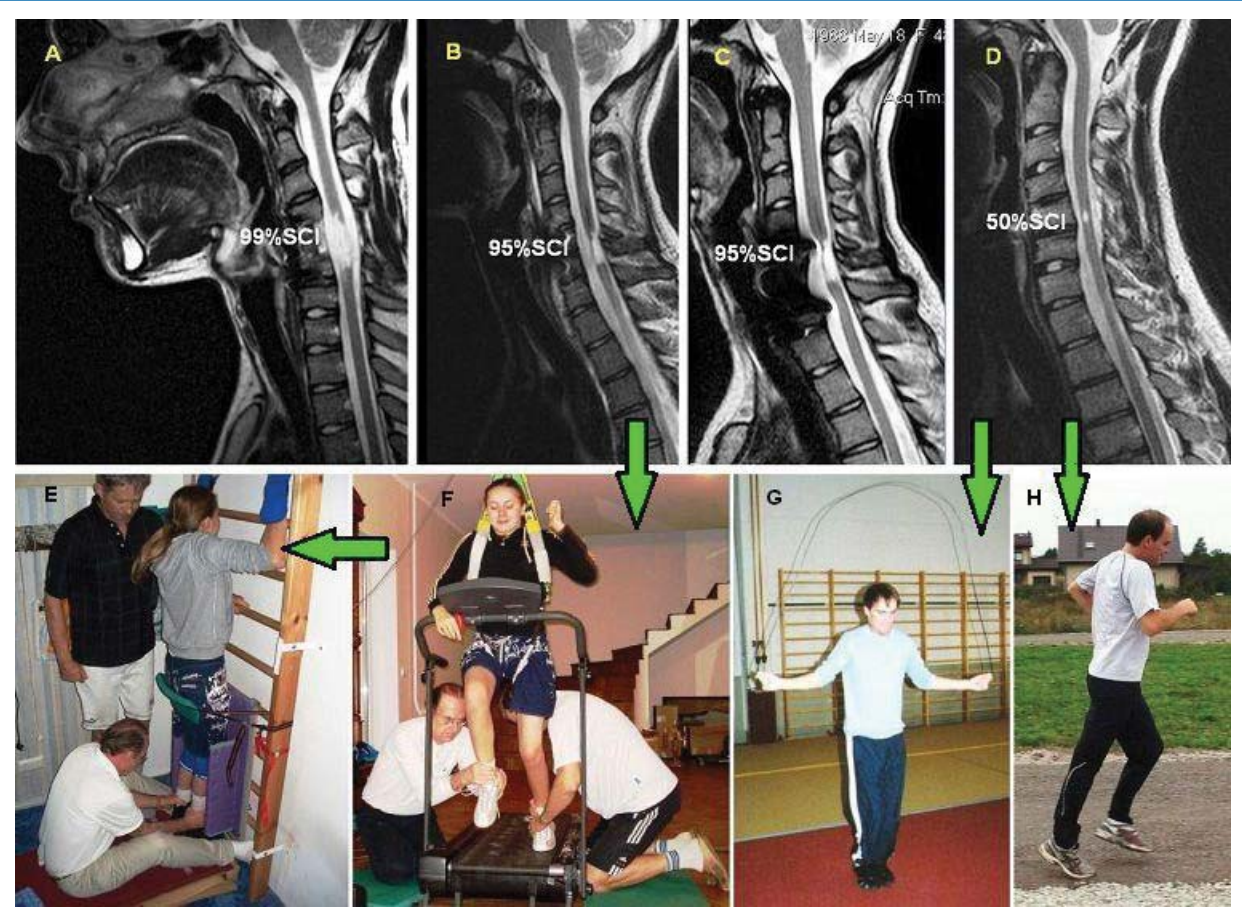

Figure 19: The outcome of a SCI repair depends strongly on the severance of the injury. A-D. MRI's of approximately $99 \%$, $95 \%$ and $50 \%$ injury. C shows the $50 \%$ injury with titan fixation and B without. The severance of an injury can also be estimated with a fixation in place. G,H. In $50 \%$ injury, the patient can relearn walking, running and jumping. E,F. In 95\% (and 99\%) the patient cannot re-learn free walking and jumping. 
3.1.2. Spinal cord repair depends on the severance of the injury : A SCI is followed by a spinal shock of approximately 2 to 4 weeks and a spontaneous recovery. Following a complete SCI there is only little spontaneous recovery and following an incomplete injury, the main spontaneous recovery takes place in the following 6 months. One year after the injury the spontaneous recovery in mainly finished. The mainly spontaneous recovery is 'sold' by rehabilitation centers as SCI repair. Efficient movement treatment has to start as early as possible, best in the spinal shock phase.

The improvement of CNS functioning depends on the injury level, the severance of the SCI and the efficiency of the treatment. The repair is mainly a functional one and is achieved by a re-wiring of the remained tract fibers. The functional repair is therefore mainly achieved by a reorganization of the brain. As more tract fibers remained as more functional repair is possible.

Figure 19 shows the CNS repair in dependence on the extent of the injury, quantified by MRI. In a $50 \%$ C5/6 injury (Figure 19D), the patient can learn to walk, run and jump (Figure 19G,H). Hand functions became quite good. Urinary bladder functions were repaired within 2 months. The patient had with some deficits a normal life. In a 95\% C5/6 injury (Figure 19B,C), the patient could not learn to freely walk and jump through 5 years of CDT. With substantial support walking and jumping was possible (Figure 19E,F). The patient had very little hand and finger functions as is typical for severe C5/6 injury, because the motoneurons are mainly destroyed in the grey matter. But the urinary bladder functions were repaired through 3 years of intensive CDT. The bladder repair is firstly the most important function to be repaired and secondly, because of nearly no finger functions, the patient cannot empty the bladder by himself through intermittent catheterization. The patient loses the intimism sphere, because somebody else has to do the catheterization. In a 99\% injury (Figure 19A), the repair situation is worse. The urinary bladder could not be repaired within 3 years of rather intensive therapy.

It is obvious from Figure 19 that possible repair depends strongly on the severance of the injury. In SCI repair the damage has to be documented.

3.1.3. Motor improvement in $\mathbf{5 0 \%}$ SCI : The 17.5 -year-old male patient Sten suffered a cervical SCI at C5-6 levels in a diving accident. The SCI was incomplete (ASIA grade D). Some sensory-motor functions were preserved below the level of injury. Initially the patient received conventional rehabilitation treatment and then CDT was started, 5 months after the injury. At the time of initiation of CDT, this patient needed a stick as support for walking, was wearing an orthosis on the left foot and emptied the bladder with intermittent catheterization. During walking, the left heel was not touching the ground. While jumping on the springboard, his feet had to be supported by the therapist. While turning on the special CDT devise, the left hand had to be fixed.

He received nearly three years of non-optimal CDT. One year after the injury, the metal support, used for spine fixation, was removed. At the end of the 3rd year of CDT, he was able to walk, run, and even jump independently and did not need any internal or external support. He was continent again and off of all medications. In that sense it can be safely said that the patient is almost cured from his quadriparesis.

In Sten with the 50\% SCI at the level of C5/6, the coordination dynamics (CD) values for $20 \mathrm{~N}$ had improved strongly after approximately one year of CDT (Figure 21). For 100N, when going deeper into the complexity of CNS organization, good CD values were reached only after 2 to 3 years. For 150N, going even more deep into the complexity of CNS organization, good baseline CD values were only achieved after 4 to 5 years of non-optimal CDT (Figure 21).

Such high-load CD values improvement should also have visible improvement in motor performance. The motor training of the patient (which included crawling, walking and running on treadmill in the forward and backward direction, jumping on spring-board,) was guided by the therapist to correct abnormal movement patterns. At the end of the 3 years of therapy, the significant improvement of coordination dynamics values (Figure 21) was accompanied by a substantial improvement in the performance of running and jumping 
with the skipping rope (Figure 19G). The times required for running 60 and 400 meters decreased and the number of jumps per series increased (Figure 20). Even later on, the patient took part in Marathon with healthy people. He was one of the last ones, but among healthy participants.

By comparing the improvement of the coordination dynamics values for higher loads $(150 \mathrm{~N})$ of the patient with a 50\% SCI (Figure 21) with those of the patient with a severe brain injury (Figure 18), it can be seen that they are similar. This means that the repair of a $50 \% \mathrm{SCI}$ is mainly a functional one and is taking place in the brain. Regeneration of tract fibers will have contributed only little.

3.1.4. Motor improvement in $95 \%$ and $99 \%$ SCI at C5/6 levels : In an almost complete cervical $\mathrm{SCI}$, the caudal spinal cord is disconnected from the cerebral cortex and brain stem. Following the spinal shock some reflexes, such as the stepping automatism and urinary bladder reflex (similar to those in infants), may re-appear, especially when stimulated. Pathologic reflexes or automatisms also appear as for example extensor spasticity. With very limited regeneration upon CDT, motor and vegetative functions partly re-appear in a cephalo-caudal direction and movements become controlled first proximally and then distally; spasticity reduces. This cephalo-caudal and proximal to distal scheme only partly holds, because the injured CNS uses all existing repair and adaptation mechanisms upon movementbased learning, including plexus connections outside the spinal cord. But with respect to the improvement of trunk stability and breathing the repair in cephalo-caudal direction seems to hold.
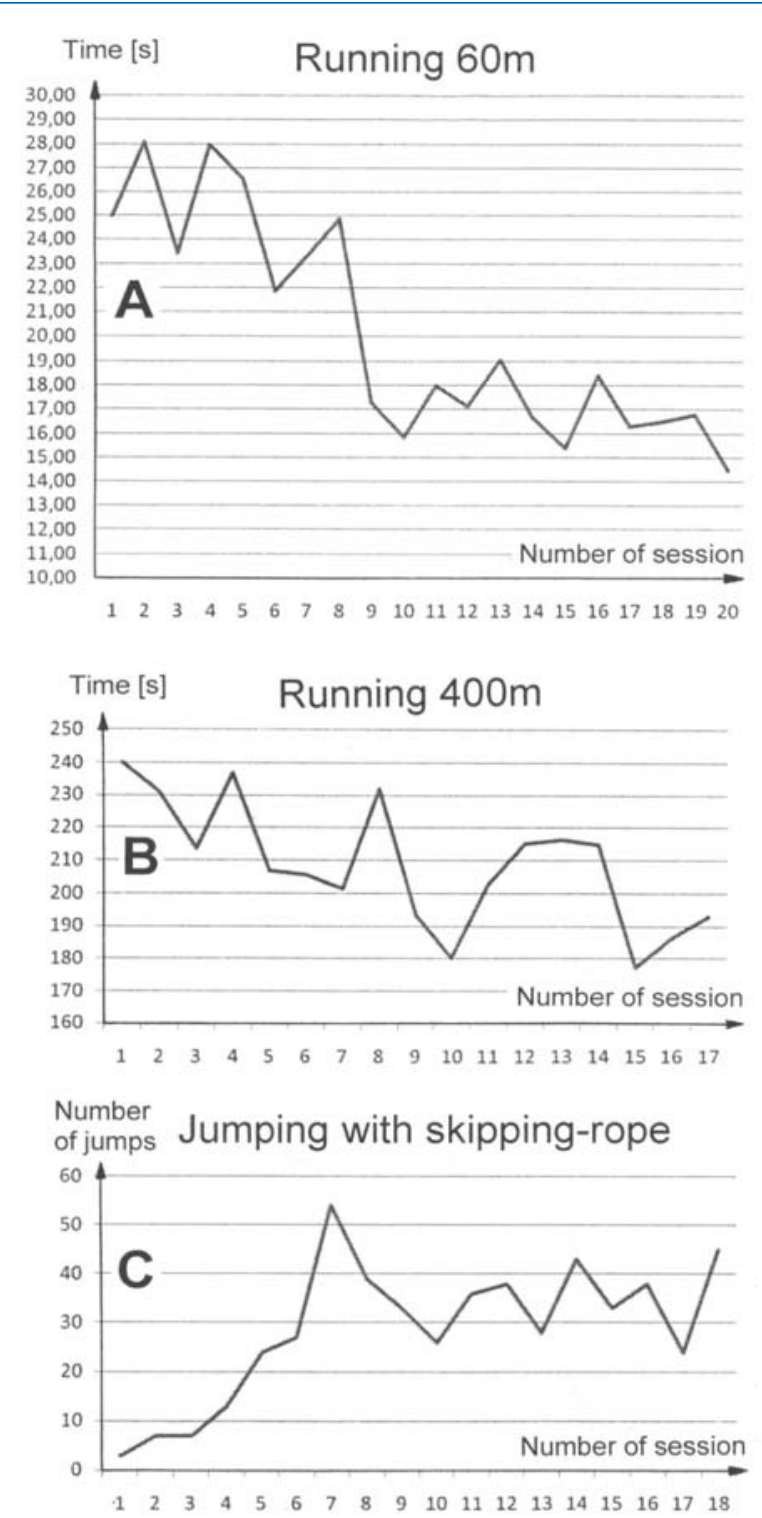

Figure 20: Improvement of times for running 60 and $400 \mathrm{~m}(\mathrm{~A}, \mathrm{~B})$ and increase of jumps with a skipping-rope per single session (C) in relation to on-going therapy sessions. Therapy period = second half of 2006 (see Figure 21).

3.1.5. Comparison between repair and development in two almost complete SCI patients with respect to administered therapy time : To improve the efficiency of SCI repair through CDT, it may be helpful to compare the improvement of trunk control and antigravity movements between development and repair. For the comparison two patients with similar severe cervical SCI were used. The MRIs are shown in Figure $22 \mathrm{~J}, \mathrm{~K}$. Through 2 years of therapy, the boy (99\% injury) mainly regained some trunk control and the breathing improved; the urinary bladder function could not be repaired within 2 years. To the young lady Kadri (95\% injury) 5 years of CDT were administered. Bladder function was repaired and many movements improved including breathing. The MRIs of the patient's SCI are shown in Figure $22 \mathrm{~J}, \mathrm{~K}$ and the two treatment stages are compared with the development in Figures 22,23.

Through one year of CDT, the patient regained little trunk control and had big problems with the trunk 


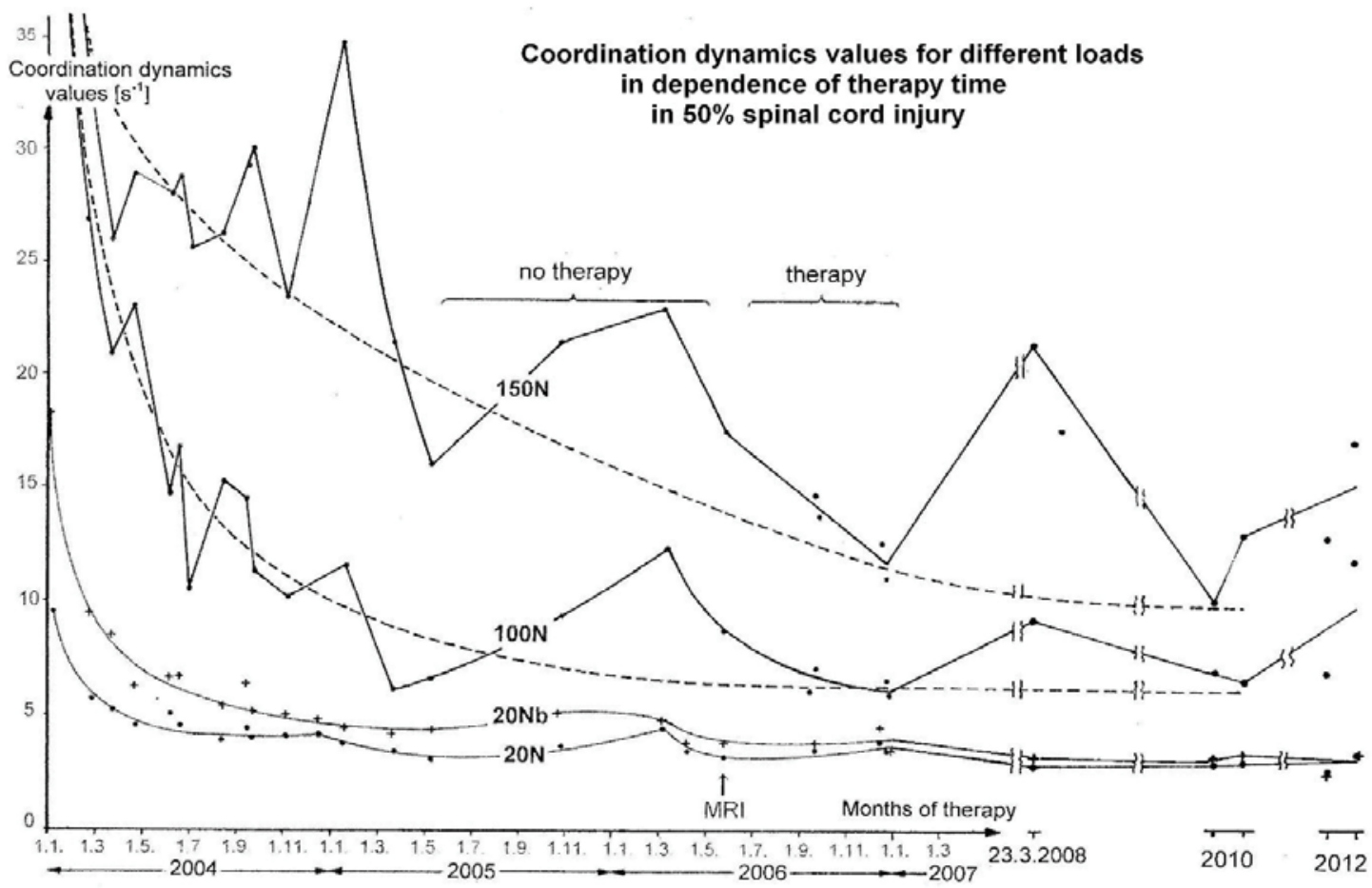

Figure 21: Relation of coordination dynamics values to therapy length for increasing load between 20 and $150 \mathrm{~N}$. The loads for forward exercising (dots, $20 \mathrm{~N}, 100 \mathrm{~N}, 150 \mathrm{~N})$ are marked at the curves $(20 \mathrm{Nb}=$ backward exercising (crosses) at $20 \mathrm{~N})$. Note that with no therapy the coordination dynamics values got worse and upon administering therapy again the values improved again even 2 years after the accident. After stopping therapy, the coordination dynamics values for 100 and $150 \mathrm{~N}$ increased again (23.3.2008). When the patient (Sten) trained himself intensively (including running and jumping), he further improved his high-load CD values (8.9. and 23.9.2010).

rotation. Arms and legs could not be flexed for the rotational movement (Figure 22A). Through 5 years of CDT, the patient Kadri could easily roll from supine to prone position and backwards. The movement performance with slightly flexed arms and legs (Figure 22B) looks much healthier and more similar to those of an infant (Figure 22C). The un-sustained sitting (Figure 22F) following 0.5 years of therapy was also very poor. No leg flexion was possible. The back was not straight and the hand could not be opened (Figure 22D). Following 5 years of therapy, the un-sustained sitting (Figure 22E) was more similar to the pattern of the infant (Figure 22F). The four-point kneeling (Figure 22I) (crawling position) was poor following one year of

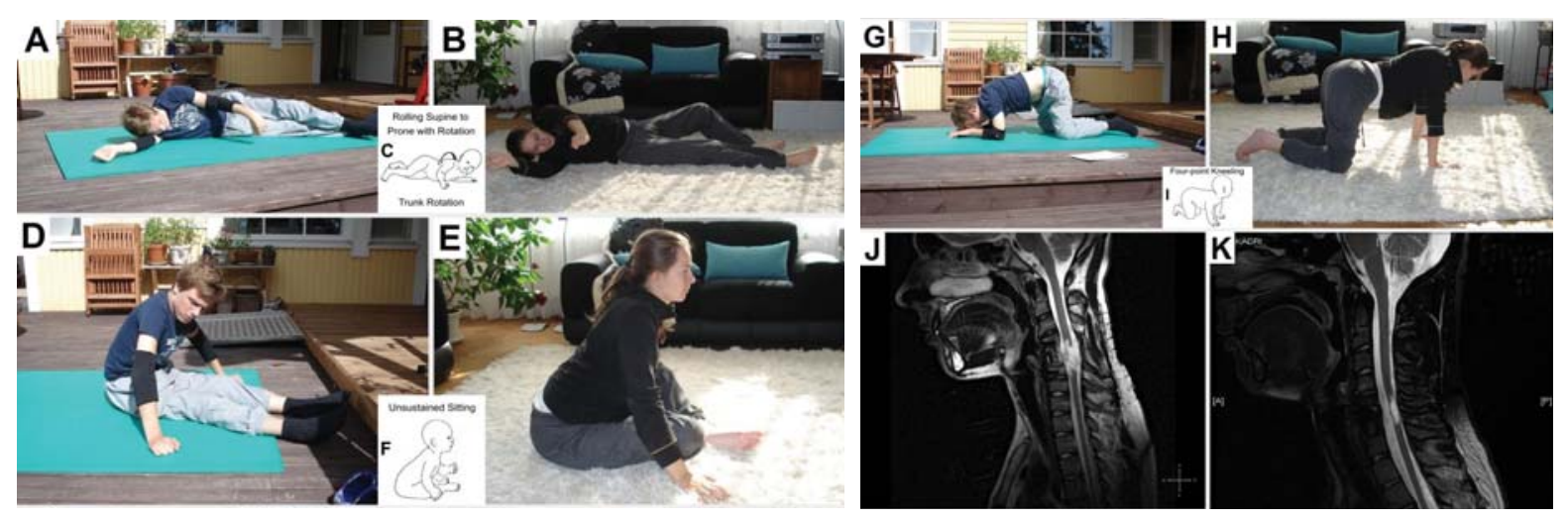

Figure 22: Comparison between development and the repair of an almost complete cervical SCI upon one year (A,C,G) (therapy started at an age of 15.5 years) and 5 years of CDT (B,E,H) (CDT started with 17 years). The MRI of the SCI of the patient of the left panel is shown in 'I' and those one of the right panel in ' $\mathrm{K}$ '. Trunk rotation $(\mathrm{A}, \mathrm{B}, \mathrm{C})$, un-sustained sitting $(\mathrm{D}, \mathrm{E}, \mathrm{F})$, and four-point kneeling $(\mathrm{G}, \mathrm{H}, \mathrm{I})$ is compared between one year and 5 years of CDT and with the positioning of an infant. 
treatment (stretched arms are not possible without support) (Figure 22G) and better following 5 years of treatment (Figure 22H). But the main weight was still on the arms (unsupported crawling was not possible). The feet and leg positioning was much better than in the other patient (Figure 22G) and the arms could be stretched.

The hands to knees positioning of the infant (Figure 23C) could be achieved in the patient upon one years of therapy (Figure 23A). The wheelchair was necessary for hands to knees touch. But this position helps the patient to exercise the pace gait coordination and co-movement can be induced in the legs by the synchronous touch of the hands. Upon 5 years of CDT, the 'hands to knees' position could be achieved (Figure 23B) and the abdominal muscles were activated, but very little power was in the legs and there was no (or not enough) dorsal flexion of the feet.

The supported standing of the infant with leg movements (Figure 23F,I) was not possible in the patients, even following 5 years of CDT. The leg muscles had still too little muscle power. But with different supports, both patients could train upright movements with full weight-bearing, such as swinging/jumping on springboard and sky-walking (Figure 23D,E). For walking on treadmill upon one year of therapy, weightbearing and feet support was needed (Figure 23G). Upon 5 years of CDT, the patient still needed weightbearing support, but was able to move the legs volitionally without support (Figure 23H), even though the
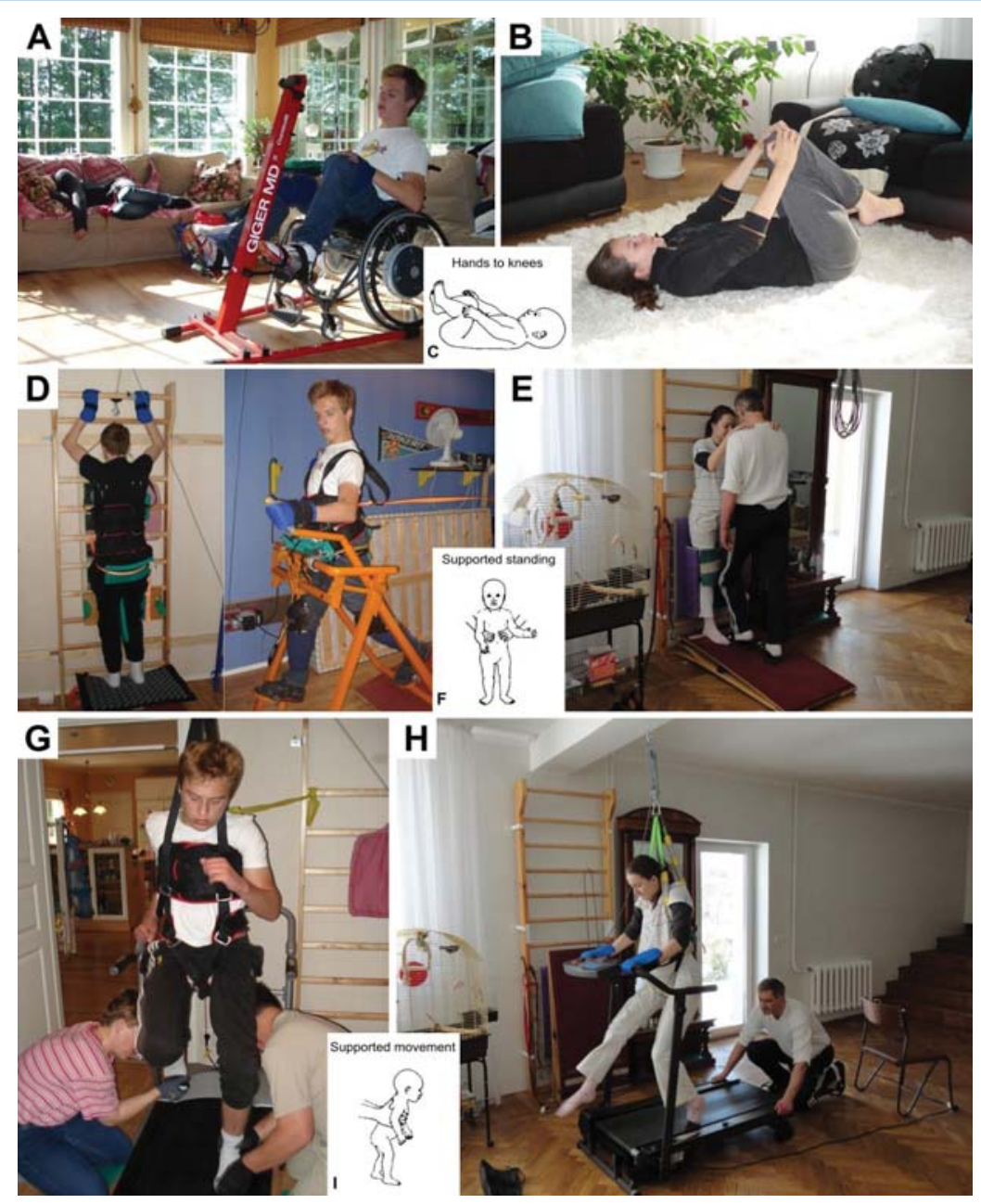

Figure 23: Comparison between the development of an infant and repair of a cervical SCI upon CDT. Left penal, positioning upon 0.5 years of therapy and right penal after 5 years of therapy. Same patients as in Figure 36. Reaching of hands to knees, supported standing, and supported walking are compared. 
dorsal flexion of the feet was missing. Upon walking bare foot on treadmill, the stepping automatism was stimulated strongly. The supported standing pattern of the infant (Figure 23I) was still a dream for both SCI patients. But development lasts 17 years. The patients could not catch up with the development, because for organizational reasons optimal CDT was stopped after 3 and 5 years respectively.

In conclusion, in 95\% and 99\% SCI's, the motor possibilities are very limited and in no comparison to the 50\% injury case. In 95\% injury, Kadri could swing a bit with the legs under weight reduction (Figure $23 \mathrm{H}$ ) and jump a bit without support of the Author (Figure 19E). In 99\% injury (Figures 23A,D,G), the patient could perform supported treadmill walking like in Figure 23F, but could not learn within 3 years to swing the legs by himself (Figure 23H). Also on floor movements, Kadri with a 95\% injury was better than the patient with the $99 \%$ injury (Figure 22). Both patients with the $99 \%$ and the $95 \%$ injury had no chance to re-learn walking like the patient Sten with a 50\% injury. Probably one needs at least $20 \%$ of the cord remained to re-learn a bit of walking.

3.1.6. Quantifying spinal cord repair in 95\% injury : In 50\% SCI it was shown that CNS repair could be quantified by the improvement (lowering) of the coordination dynamics (CD) values (Figure 21) when exercising on the special CDT device. In 95\% injury at the level of C5/6, the patients do not have sufficient muscle power to exercise at higher loads.

Still it is helpful to record the CD values, because it can be seen that with every improvement of CNS functioning the $\mathrm{CD}$ values get worse, due to the transient impairment of the phase and frequency coordination following neural network change, before they get better again through therapy (Figure 24). With every bit of repair or change of neural networks, the CD values get transiently worse.

3.1.7. Comparison of urinary bladder repair between $\mathbf{5 0} \%$ and $\mathbf{9 5 \%}$ spinal cord injury : The most important functions to be repaired in SCI are firstly the bladder repair, followed by the sexual function. The walking is on place 3 followed by spasticity and scoliosis.

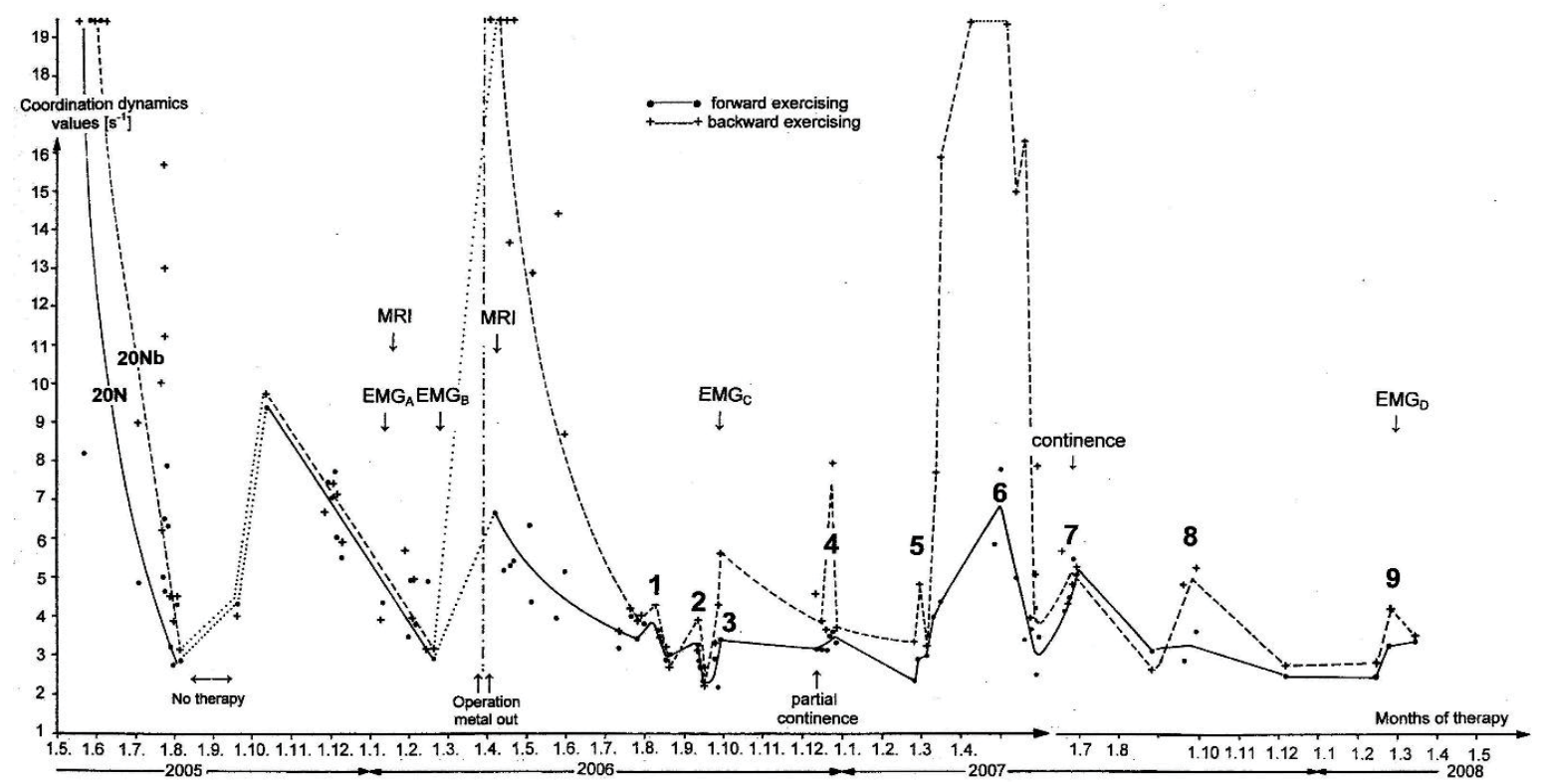

Figure 24: Relation of coordination dynamics values to therapy duration for a load of $20 \mathrm{~N}$ and for exercising in the forward (lines and dots) and backward directions (20Nb; dashed line and crosses) in a patient with a SCI sub C5/6 (Kadri). Note that with no therapy the coordination dynamics values got worse (increased) and upon therapy they improved again. Upon metal removal the coordination dynamics values increased strongly. The transient coordination dynamics value increases (peaks) ' 1 ' through ' 9 ' fall together with the re-appearance of certain muscle functions or specific improvements of motor and autonomic functions and indicate therefore most likely small bits of regeneration. After the large peak ' 6 ' of transient coordination dynamics value increase, urinary bladder functioning was re-learned. 
In the patient with a $50 \%$ SCI (Sten) the bladder was fully repaired through 2 months of CDT. In the patient Kadri with a 95\% SCI for the repair through CDT 2.5 years were needed. In the 99\% injury the bladder was nor repaired within 2.5 years.

Because in 50\% SCI the bladder is somehow functioning, the animal researcher cut only 50\% of the cord in animals to avoid urinary bladder problems, even though it is the most important function to be repaired in SCI.

3.1.8. Clinical urinary bladder function test (Urodynamics) : The functioning of the urinary bladder can be evaluated by measuring the pressure in the bladder and in the abdomen (colon) and the electromyographic (EMG) activity of the external sphincters and/or functionally associated pelvic floor muscles. Such bladder diagnosis is called urodynamics (Figure 25). Especially the simultaneous activation of the detrusor (line detrusor pressure in Figure 25B) and the sphincteric and/or pelvic floor muscles (line pelvic floor EMG in Figure 25B), the so-called detrusor-sphincter-dyssynergia, can be measured, which may destroy the kidneys on the long term.

Upon retrograde filling of the bladder in a patient with a SCI, the pressure in the bladder and colon is measured and the electromyographic activity (EMG) of the pelvic floor is recorded with surface electrodes (Figure 25). The detrusor pressure is obtained by subtracting the abdominal pressure from the bladder pressure. The continence status of the patient is diagnosed by the reports of the first feeling of bladder fullness, the desire to void, and the leaving of fluid out of the bladder. The EMG activity of the pelvic floor informs when the external bladder and anal sphincters are activated. Upon knocking, pressing, coughing, and stimulating bladder reflexes, the bladder status is obtained. This patient of Figure 25 with a SCI subTh12 had a detrusor-sphincteric-dyssynergia of the bladder, because the EMG activity of the external sphincters increased (the sphincters became activated) with the increase of the detrusor pressure (activation of the detrusor). Improvement of urinary bladder function mainly due to therapy can be quantified by repeated urodynamics measurements (Figure 25AB).

3.1.9. Limitation of urodynamics and need for human electrophysiology for causal repair : The evaluation of bladder functioning by means of urodynamics gave the information that the patient had a dyssynergia of the bladder. Repeated testing informed about changes in bladder functioning. But such bladder function tests are giving no information on the pathology of the CNS organization and how to repair urinary bladder functioning causally.

By performing similar bladder tests under operational conditions and recording single-nerve fibre action potentials from sacral nerve roots (Figure 6), the pathology of CNS organization can be explored in patients with SCI and ideas can be found how to repair the injured CNS causally. Since humans have a high capability for learning it is possible to repair the injured networks by learning and learning transfer from exercising on the special CDT device and jumping. By comparing bladder and motor functions at the neuron level, it may be understandable why learning transfer from movements to bladder functions is possible.

3.1.10. Bladder functioning at the neuron level: With the single-nerve fiber action potential recording method (Figure 6) it has so far been possible to record single-nerve fiber action potentials from nerve fibers down to a diameter of approximately $3.5 \mu \mathrm{m}$ in undissected thin long sacral nerve roots/fascicles. It has therefore also been possible to record natural impulse patterns from parasympathetic efferents (par) (Figure 26), urinary bladder stretch and tension receptor afferents (S1, ST), mucosa afferents from mechanoreceptors of the bladder, the urethra and the anal canal (M), from afferents responding to fluid movement (S2), and from $\alpha_{2}, \alpha_{3}$ and $\gamma$-motoneurons and muscle spindle afferents innervating the external striated urinary bladder and anal sphincters (or functionally associated pelvic floor muscles), and to analyze regulatory and organizational mechanisms of parasympathetic neurons and motoneurons in the human CNS (Figure 27). 

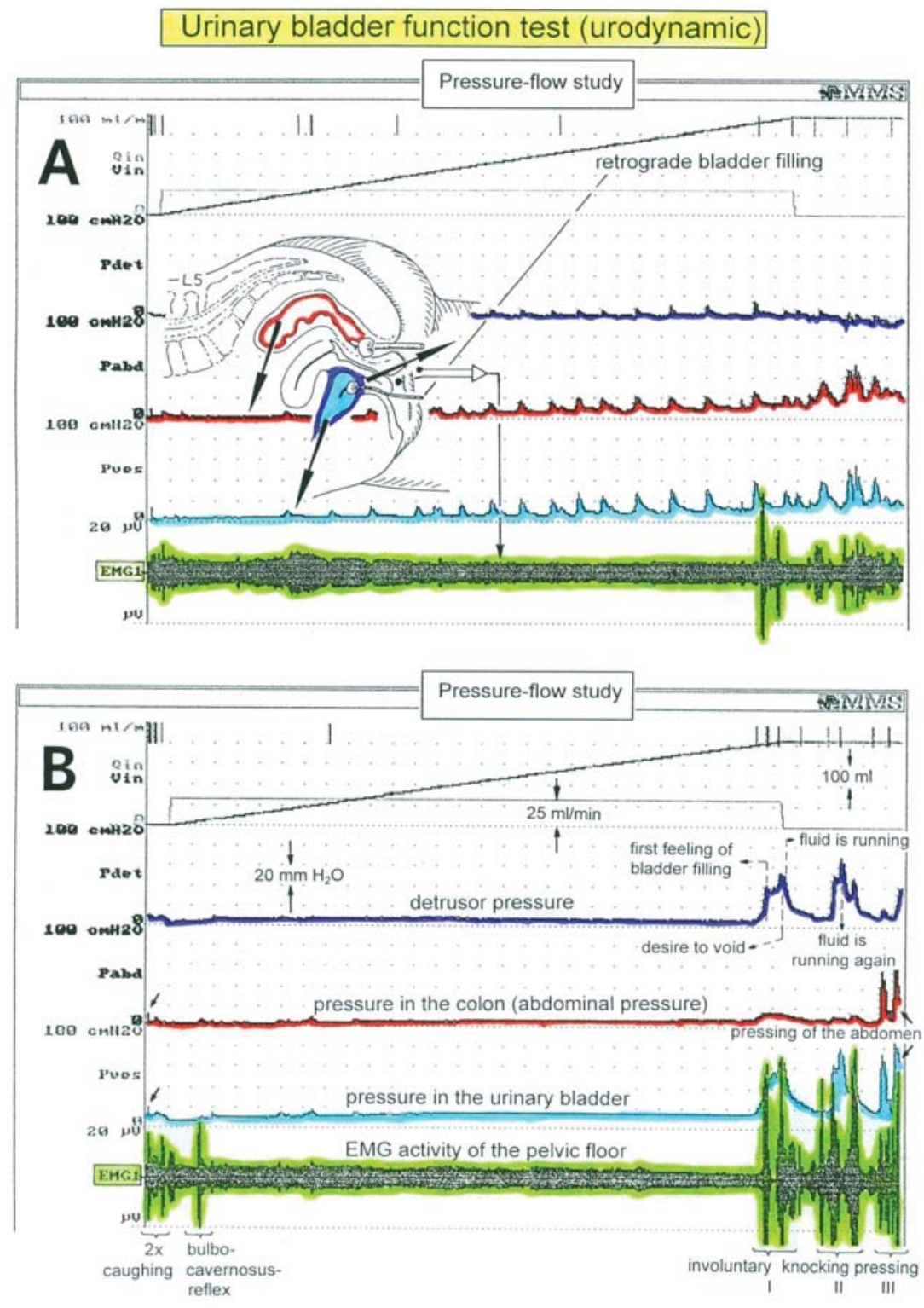

Figure 25: Clinical urinary bladder function test (Urodynamics). Improvement of the urinary bladder functions, quantified by urodynamics in a 30 -year-old female patient. A. 3 months after the accident resulting paraplegia sub Th12 following spinal cord lesion. B. 12 months after the accident (lesion level lowered to sub L3). In A, the detrusor pressure (Pdet) is generated by the contracture of the bladder wall, as the pressure difference between abdominal pressure (Pabd, measured in the rectum) and the bladder pressure (Pves, measured in the bladder). Electromyographic recording obtained with surface electrodes from the sphincters and the pelvic floor (EMG) is shown; the external sphincters and the functionally correlated pelvic floor muscles show similar SEMG activity (the rhythmic pressure peaks in A do not originate in the bladder). In A, the detrusor shows nearly no activity with retrograde bladder filling at $25 \mathrm{ml} / \mathrm{min}$; in B, the detrusor shows first activity at $360 \mathrm{ml}$ bladder filling. A detrusorsphincteric-dyssynergia occurs, because the detrusor pressure peaks occur at the same time as the sphincter EMG activity peaks (B) (bladder and sphincter contract at the same time, so that fluid can only emerge from the bladder at high bladder pressure; there is a danger of reflux through the ureter into the kidneys). The EMG peaks are a bit irregular, probably because the fluid, leaving the bladder, shunts transiently the EMG electrodes. Exact functional description of B: 2x coughing (B below) increases the EMG activity and passively the pressure in the abdomen and in the bladder (marked by the small arrows, physiologic). The bulbocavernosus reflex (induced by pressure applied to the clitoris) increased the EMG activity of the sphincters (physiologic). Conclusion: The reflex arch is in order; sacral nerve roots and nerves have not been damaged in the accident. I (bottom right): The patient feels an increase of unvolitional detrusor pressure (first feeling of bladder pressure at $360 \mathrm{ml}$ ). She tries to contract the sphincters to stop the bladder emptying. Shortly after the desire to empty the bladder, as the detrusor pressure decreases, fluid is leaving the bladder. II: Due to tapping onto the bladder, the bladder reflex is activated (detrusor activated, nearly no abdominal pressure); fluid is leaving the bladder. III: Due to the abdominal muscular pressure the pressure in the abdomen increases as does passively the pressure in the bladder (the detrusor is not activated); fluid left the bladder. With a delay, the detrusor was activated by the bladder reflex. - The urinary bladder of the patient is partly functioning. It has to be further improved by therapy induced reorganization of the CNS: (1) An earlier feeling of bladder filling, (2) an increase of the time difference between the feeling of the first bladder filling and the un-volitionally emptying of the bladder (for the time being, approx. 10 min, in dependence on whether the patient is physically active (such as walking) or not, (3) further learning how to activate the detrusor on volition, and (4) the physiologic coordination between the bladder and the external sphincter functioning (to stop the detrusor-sphincter-dyssynergia). 
It has been reported that the sacral autonomic outflow is sympathetic [26]. This report is not fully convincing, because in the publication electrophysiological (functional) recordings are missing. But if the sacral autonomic outflow is really sympathetic, this would not change the considerations, when just replacing the word parasympathetic by sympathetic.

Following a laminectomy, natural impulse traffic (somatic and autonomic) to and from the CNS can be recorded from sacral nerve roots (Figure 26). The summed impulse traffic can be split into the natural impulse patterns of single afferent and efferent fibers. Upon retrograde bladder filling as in clinical diagnostic (Figure 25) and the identification of the neuron type, with the use of the classification scheme for human nerve fibers (Figure 9), the natural impulse patterns of identified afferent and efferent fibers can be obtained and analyzed. The obtained natural impulse patterns running in and out of the CNS answered an old question: Is it the firing rate of a neuron that codes the information transmitted and processed or does the precise timing of cell discharge codes information? It is shown here that the information from and to the periphery is coded by specific impulse patterns, including the precise timing and the firing rate.

In response to retrograde bladder filling as during urodynamics (Figure 25) of a brain-dead human, the self-organization of a premotor spinal $\alpha_{2}$-oscillator innervating the external striated urinary bladder sphincter is shown in Figure 28D. Because the motoneuron axon of 01 had a recurrent fiber (Figure 28A) at the recording site, each single AP of this motoneuron could be identified safely by the action potential (AP) of the recurrent fiber. The function of the motoneuron was to secure bladder continence. The activity from urinary bladder receptors, i.e. the activity of bladder stretch (S1), tension (ST) and flow receptor afferents (S2) (Figure 28E), was an adequate afferent input to the motoneuron. Phase relations between the firing patterns of bladder afferent fiber S1(1) and oscillatory firing urinary bladder sphincteric motoneuron 01 can be seen in the schematized firing patterns in Figure 28B. They were an indicator that the natural impulse pattern of the S1(1) fiber was an adequate drive of the sphincteric motoneuron 01. For retrograde bladder filling up to $550 \mathrm{ml}$, motoneuron 01 only fired occasionally (Figure 28D,F). This was the storage phase of the bladder, during which the intravesical bladder pressure increased only little. For higher bladder filling volumes, the motoneuron switched via the transient oscillatory firing mode to the continuous oscillatory firing mode (Figure 28D) to generate a higher activity level (Figure 28F) for a stronger drive of the urethral sphincter to more strongly secure continence when the storage phase was nearly passed and the bladder pressure increased. For bladder filling volumes higher than $800 \mathrm{ml}$, the activity of the spinal oscillator decreased again; probably, the oscillator became inhibited (Figure 28D). Pain fibers (not shown in Figure 28G) may have inhibited the oscillatory firing to protect the bladder from mechanical damage. The overflow mechanism was initiated. Probably, fluid entered the trigonum vesicae to activate flow receptors, so that the flow receptor activity (S2) increased strongly (Figure 28E). The premotor spinal oscillator 01, of which motoneuron 01 is probably a part, was organized by the adequate afferent input (S1, ST, S2, ...) induced by bladder filling.

By recording from the dorsal S4 root of the brain-dead human HT6, impulse trains from another oscillatory firing motoneuron and its driving afferents were measured, which served quite a different function (Figure 28F). The $\alpha_{2}$-oscillator 02 (Figure 28A,B) innervating the striated external anal sphincter was activated by secondary muscle spindle afferent activity, induced by the anal catheter-stretched muscle spindles, probably located in the anal sphincter or functionally associated pelvic floor muscles. Also, mucosal and skin receptors within the anal reflex area will induce self-organization of premotor oscillators activating the external anal sphincter to secure anal/rectum continence. It is evident from the impulse patterns, shown schematically in Figure 28B, that the impulse trains of this oscillator 02 show a phase relation and an interspike interval relation to its driving spindle afferent APs. But no synchronized firing can be seen between the sphincteric $\alpha_{2}$-motoneurons, innervating the external bladder sphincter 
Sympathetic and Parasympathetic nervous system

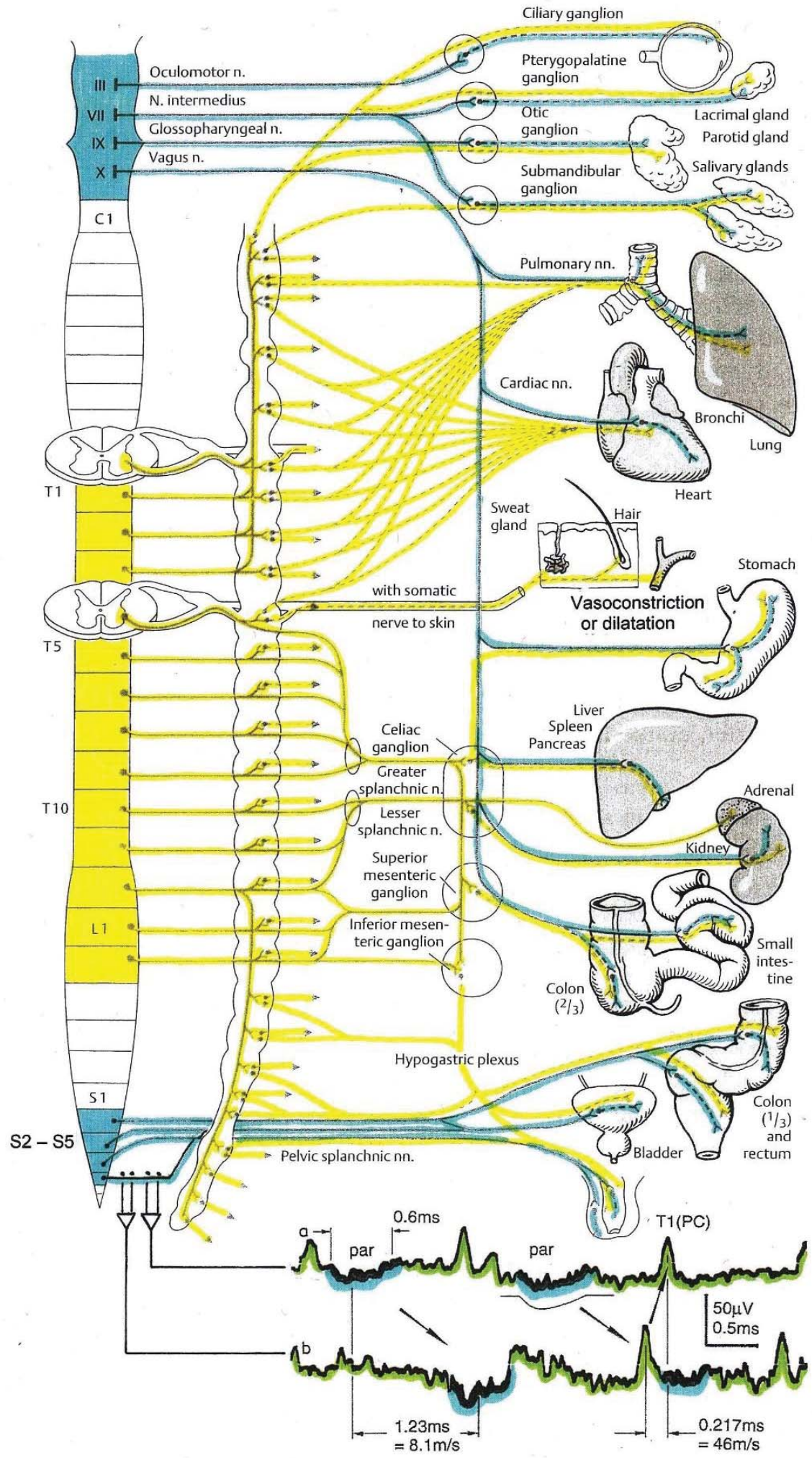

Figure 26: Schematic diagram of the sympathetic and parasympathetic nervous system. Yellow = sympathetic, blue = parasympathetic. The recording from a sacral root shows single action potentials of preganglionic neurons (par) and a skin afferent fiber. 


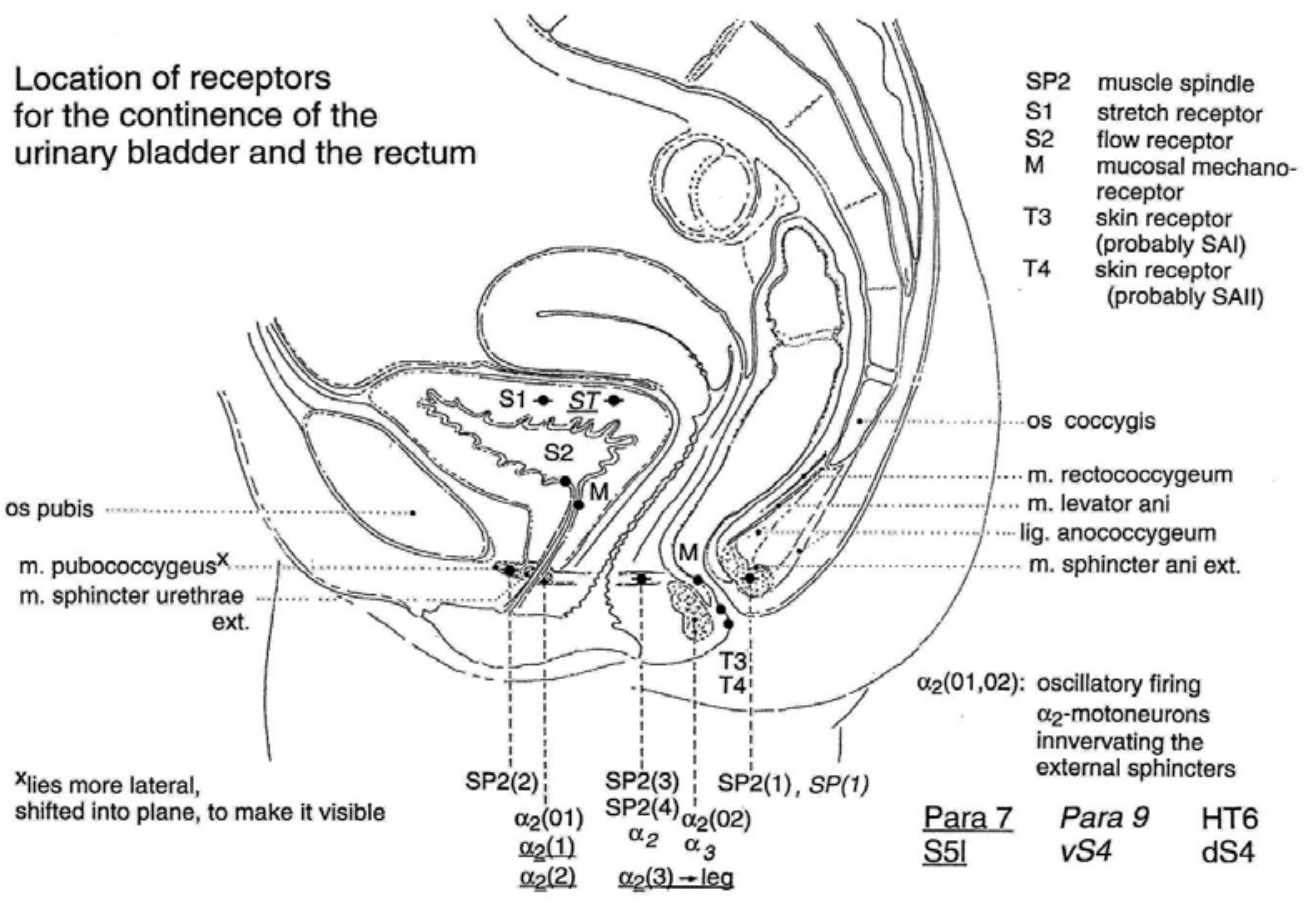

Figure 27: Location of receptors and muscles for the continence of the urinary bladder and the rectum, innervated by motoneurons the activities of which were recorded, in the brain-dead human HT6 (dS4 root), paraplegic 9 (vS4 root) and paraplegic 7 (nerve root S5).

(01) and anal sphincter (02). Probably the CNS networks tried to avoid an increased physiologic tremor. Important for the application of human neurophysiology to neurotherapy is the duality of the functions of the sphincteric motoneurons and secondary muscle spindle afferents, sub-serving somatosensory and autonomic (parasympathetic) functions. In animals it was found that also sympathetic fibers innervate intrafusal muscle fibers in muscle spindles [27]. It is likely that also parasympathetic efference innervate intrafusal muscle fibers in muscle spindles in the parasympathetic innervation area (S2-S5).

Motoneurons innervating the external sphincters of the bladder and the anal canal sub-serve somatic functions (contraction of the sphincters on volition or for protection reaction) and parasympathetic functions for the coordination of the detrusor function (parasympathetic) and the external sphincter function (somatic). This duality of the sphincteric motoneurons (and spindle afferents in the parasympathetic domain) makes the pattern change from continence to protection reaction understandable and makes learning transfer between somatic and parasympathetic patterns likely. The motoneurons build up two phase relations per oscillation cycle with other motoneurons and secondary spindle afferents for somatic activation [28], and build up 3 phase relations per oscillation cycle when also the parasympathetic division is activated [28]. The neuronal networks of the somatic and the parasympathetic nervous systems are interlaced and interact with each other. It should therefore be possible to improve parasympathetic functions when improving somatic functions by CDT (neural network learning [19]), especially as there is indication that also parasympathetic efferents fire rhythmically [29].

The repair of urinary bladder functioning by reorganization of networks seems to be most difficult because there is false neuronal network organization in the parasympathetic nervous system (overactive (spastic) detrusor) and the somatic division (spastic external (striated) bladder sphincter), and there is false interaction of the interlacing somatic and parasympathetic networks (detrusor-sphincteric dyssynergia: when the detrusor contracts, the external sphincter is also contracting instead of opening (relaxing)) [2931]. 

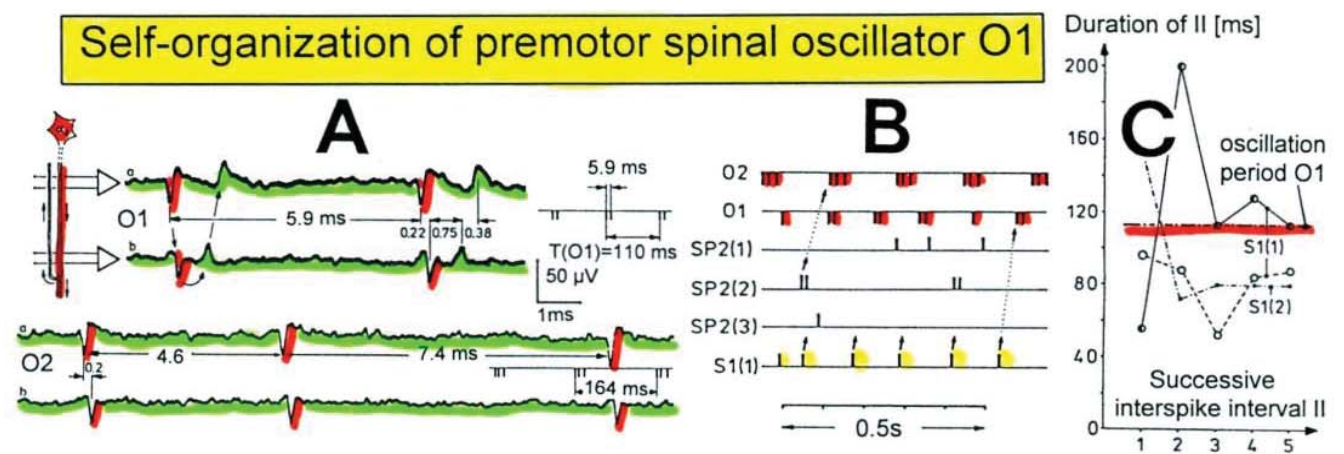

\begin{tabular}{|c|c|c|c|c|}
\hline \begin{tabular}{|c}
$\begin{array}{c}\text { Fluid in bladder } \\
{[\mathrm{ml}]}\end{array}$ \\
\end{tabular} & $\begin{array}{l}\text { Activity pattern } 01 \\
{[\mathrm{~ms}]}\end{array}$ & $\begin{array}{l}\text { Oscillation frequency } \\
\text { [impulse trains/s] }\end{array}$ & $\begin{array}{l}\text { Mean activity } \\
\text { [APs / s] }\end{array}$ & Firing mode \\
\hline 200 & & & 0.5 & \\
\hline 500 & $\sigma$ & & 1.0 & spike mode \\
\hline 550 & E & 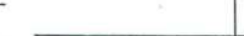 & 2.5 & \\
\hline anal-stimulat. & -11445 & (6.9) & & transient \\
\hline 580 & long short $\rightarrow$ & $(8.9)$ & 5,0 & oscillatory mode \\
\hline 620 & 794301140 & 7.8 & 12.0 & \\
\hline 700 & $7 H_{136} 16_{116} \mathrm{~m}$ & 8.0 & 14.0 & continuous \\
\hline 750 & TQ12 11109111 & 9.0 & 18.1 & oscillatory mode \\
\hline 800 & 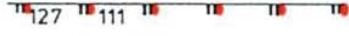 & 8.4 & 16.8 & \\
\hline 830 & $74^{15} 4^{\circ} 124^{16} 104^{\prime \prime}$ & 7.8 & 13,8 & \\
\hline
\end{tabular}
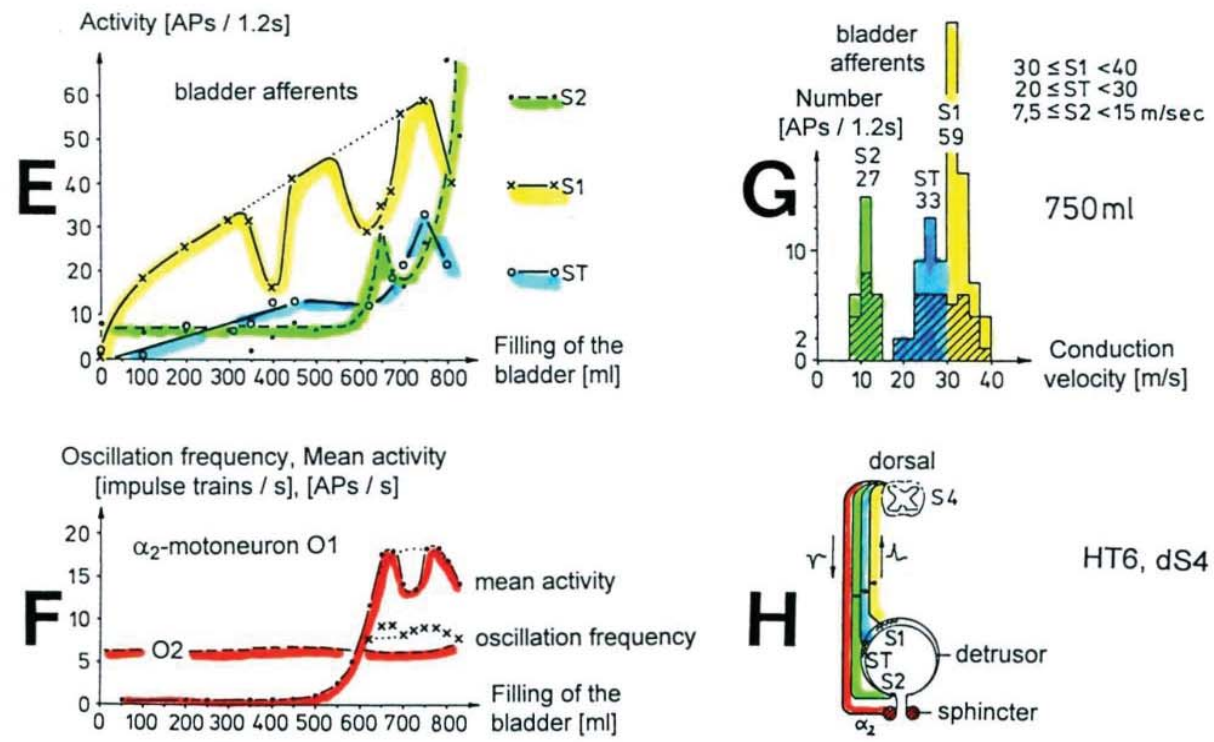

Figure 28: Self-organization of premotor spinal $\alpha_{2}$-oscillator 01 and 02, which innervates the external urinary bladder sphincter (skeletal muscle). Brain-dead human HT6; recording from a dorsal S4 nerve root.

A. Recordings from $\alpha_{2}$-motoneurons 01 and 02, firing in the oscillatory mode with impulse trains of 2 (upper recording) and 3 (lower recording) action potentials (APs). The durations of the oscillation periods were $110(01)$ and $164 \mathrm{~ms}(02)$. The interspike intervals of the impulse trains were $5.9 \mathrm{~ms}(01)$ and 4.6 and $7.4 \mathrm{~ms}(02)$. Motoneuron 01 conducted at $36 \mathrm{~m} / \mathrm{s}$; its recurrent fiber conducted at $21 \mathrm{~m} / \mathrm{s}$. The measurement layout is shown schematically. The inserts show the oscillatory firing modes; they have not been drawn to scale.

But how can the neural networks be repaired, so that the bladder is functioning physiologically again and SCI and other patients have a rather normal life again. As will be shown below, this will be achieved through neural network repair [19] induced by movement-based learning (CDT) and learning transfer [23] from movements to urinary bladder functions. The most efficient movements to cure urinary bladder functions [9] are exercising on the special CDT device and jumping on springboard. But before coming 
to the repair of the bladder, more knowledge is needed concerning the neural network organization for physiologic bladder functioning.

3.1.11. Time course comparison between the proposed parasympathetically induced muscle spindle afferent activity and the detrusor pressure : In detrusor-sphincter dyssynergia of the urinary bladder, the somatic external bladder sphincter is activated at the same time as the detrusor (smooth muscle) with the consequence that the bladder cannot be emptied. This dyes-coordination between the somatic and parasympathetic nervous system divisions in the human sacral micturition center is reflected urodynamically (clinically) in the simultaneous increase of the detrusor pressure and the electromyographic activity of the pelvic floor (Figure 25). Before analyzing the detrusor-sphincter dyssynergia at the neuron level an important correlation between muscle spindle activity and detrusor pressure, measured urodynamically, has to be done, namely that muscle spindles, also driven by the autonomic nervous system, show very similar activity changes than the detrusor pressure.

It was shown that the activity in parasympathetic efferents can be measured, identified and distinguished from the activity of $\gamma$-motoneurons in conduction velocity distribution histograms. Since the action potential amplitudes of parasympathetic efferents is small (Figure 26), it would still be very difficult to analyze the organization of the parasympathetic nervous system and it's coordinated functioning with the somatic nervous system as in the control of the urinary bladder. But some secondary muscle spindles in the parasympathetic range are also innervated by parasympathetic efferents besides somatic efferents ( $\gamma$-motoneurons). In animals it was found that also sympathetic fibres innervate muscle spindles [27]. The activation of the parasympathetic nervous system can therefore also be measured by the activity of secondary muscle spindle afferents. Since the action potentials of secondary spindle afferents are comparably large (thick fibers), the activation of the parasympathetic nervous system can be easily indirectly assessed. It will be shown now that at least some secondary muscle spindles in the parasympathetic range are innervated by parasympathetic efferents (parasympathetic fusimotors, with the above reassignment sympathetic fusimotors). The evidence is obtained by measuring the parasympathetic activation of the detrusor by detrusor pressure changes and by measuring activity changes of secondary muscle spindle afferent fibers and compare the form of the changes of detrusor pressure with the activity changes of a secondary muscle spindle afferent fiber.

In Figure 29, the increase in detrusor pressure upon retrograde bladder filling before surgery is compared with the activity increase of the secondary muscle spindle afferent fiber SP2(1) during the operation following 4 times bladder catheter pulling. Figure 29A shows the undulating activity increase of a SP2(1) fiber. In Figure 29C the cystogram is shown. Upon bladder filling spontaneous micturition occurred several times. If parasympathetic fibers really activated muscle spindles, then the activity increase of the secondary muscle spindle afferent fibers following bladder catheter pulling may have a similar time course as does the bladder pressure increase due to detrusor activation following retrograde bladder filling. To check this similarity of time course, one undulating increase of detrusor pressure (Figure 29C) has been brought to the same time scale as the measured changes in muscle spindle afferent activity (Figure 29A) and transferred into Figure 29B for a direct comparison with Figure 29A. By comparing Figure 29A with Figure $29 B$, it can be seen that the occurrence of activity peaks of secondary spindle afferents is very similar in its time course to that of the peaks of the detrusor pressure. From the similarity of changes of spindle afferent activity and detrusor pressure (4 peaks) it can be concluded that some muscle spindles in the domain of the sacral parasympathetic nucleus are partly controlled by the parasympathetic division and that the muscle spindle and the detrusor activation have similar time courses.

Thus, there is indication that some muscle spindles are partly driven by the parasympathetic division. The drive can be by parasympathetic fusimotor activity or more indirectly by somatic fusimotors. Since in HT6, the somatic fusimotors did not change their activity levels strongly with the activation of preganglionic parasympathetic fibres and the activity increase of the secondary muscle spindle afferent fibre SP2(2) followed the transient increase of parasympathetic activity, it is likely that some muscle spindles are directly controlled by parasympathetic fusimotors. For further details see Chapter V of [17]. 


\section{Parasympathetic muscle spindle activation in comparison to urinary bladder pressure}

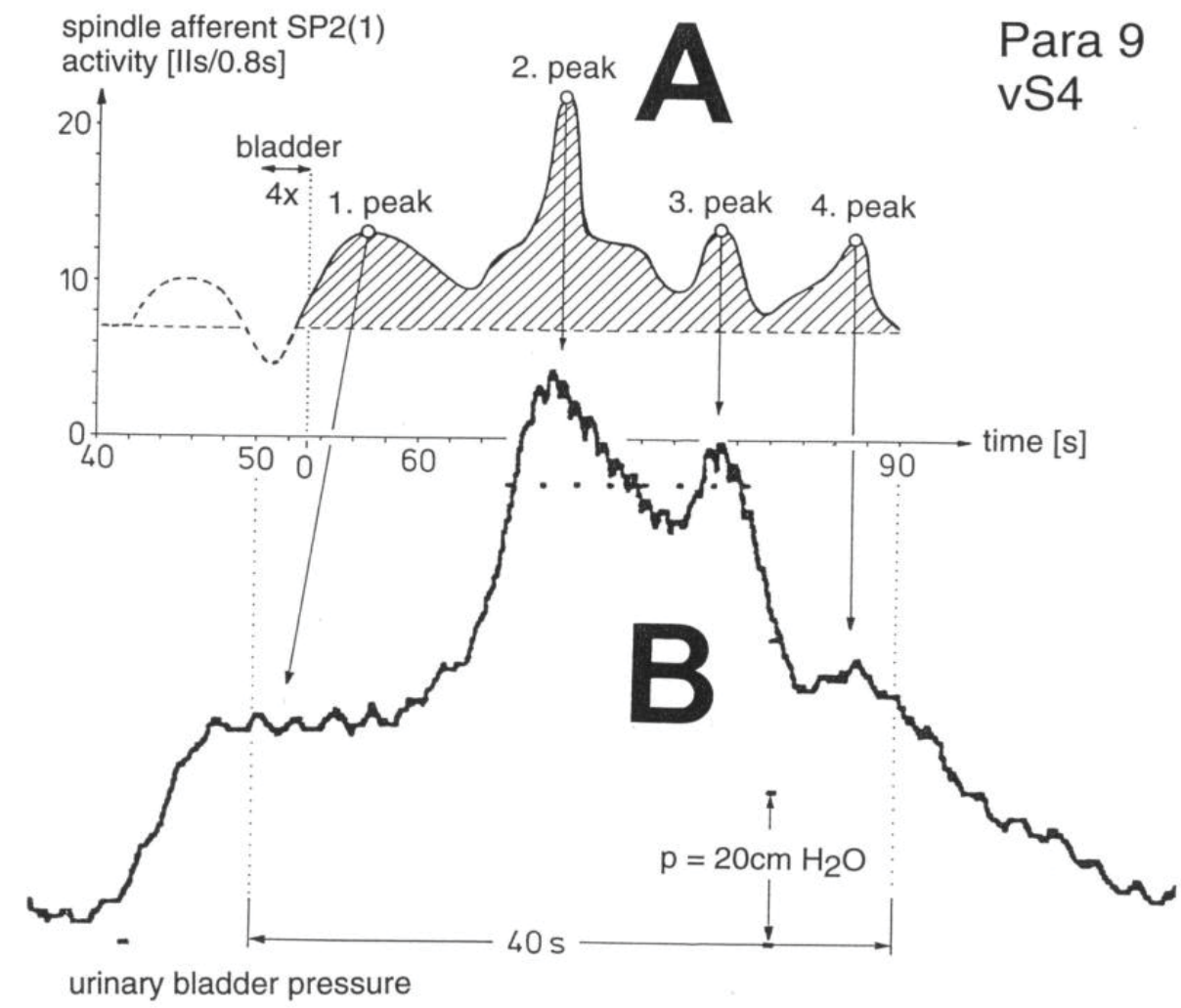

$90 \mathrm{ml}$ filling volume, filling speed $13 \mathrm{ml} / \mathrm{min}$

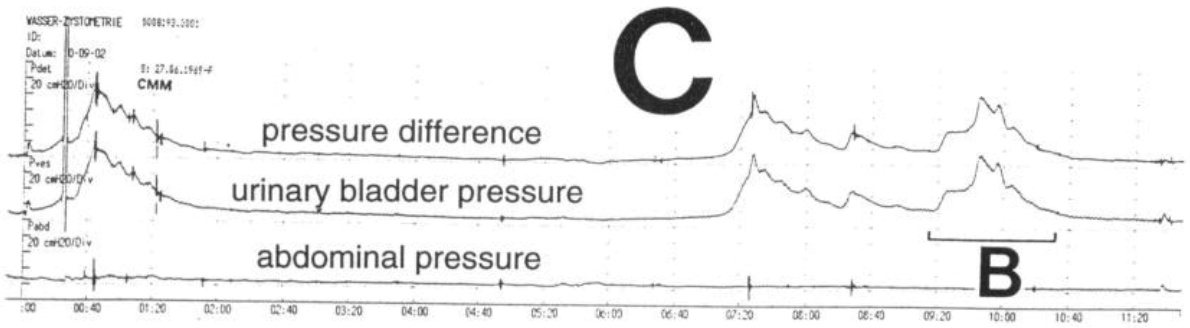

mavix

stop of

filling

retrograde filling

$\uparrow$

spontaneous

$t$ micturition

stop of

$13 \mathrm{ml}$

$58 \mathrm{ml}$

filling

$90 \mathrm{ml}$

Figure 29: Comparison between changes in secondary muscle spindle afferent activity and detrusor pressure (measured before the surgery; for the implantation of a sacral anterior root stimulator). Paraplegic 9.

A. Activity changes of the afferent fiber SP2(1) following bladder catheter pulling (Figure 37Ba). Approx. mean activity level is represented by a dashed line at $7.5 \mathrm{IIs} / 0.8 \mathrm{~s}((\mathrm{APs}-1) / 0.8 \mathrm{~s})$. The activity above the mean is cross-hatched and is proposed to be due to parasympathetic activation. Root vS4.

B. Detrusor pressure (pressure difference) changes taken from ' $C$ '. Note that changes in the detrusor pressure show almost exactly the same time course as do the activated changes of the secondary muscle spindle afferent fiber SP2(1) of 'A'. Corresponding peaks are correlated by arrows.

C. Abdominal pressure (measured as rectal pressure), intravesical pressure (urinary bladder pressure) and detrusor pressure (pressure difference) during retrograde filling before the surgery. One transient detrusor pressure increase, marked 'B', is used, after enlargement in ' $\mathrm{B}$ ', to compare with the spindle afferent activity. 
3.1.12. Detrusor-sphincter synergy of the bladder in the brain-dead human HT6 and dys-synergy in paraplegic 9 : The measurement of parasympathetic activation of the detrusor by activity changes of secondary muscle spindle afferent fibers (the spindle is innervated by autonomic fusimotors) allows an analysis of detrusor-sphincter dyssynergia using the natural simultaneous impulse patterns of secondary muscle spindle afferents and sphincter $\alpha_{2}$-motoneurons (and $\gamma$-motoneurons).

Figure 30A shows that in the brain-dead human HT6, whose parasympathetic preganglionic neurons increased activity (Figure 30Ab) upon bladder catheter pulling, the SP2(2) fiber activity increased strongly (Figure 30Aa), whereas the $\alpha$ 2-motoneuron innervating the external anal sphincter discontinued its oscillatory firing (Figure 30Ac), which is a measure for a strong activity decrease. An $\alpha_{2}$-motoneuron, innervating the external (striated) bladder sphincter, was not activated. This means that with the activation of the detrusor the sphincter motoneurons were relaxed by inhibition. Thus, the brain-dead human HT6 had a detrusor-sphincter synergy of the bladder.

In paraplegic 9 who showed a strong activity increase of the SP2(1) fiber, there was no sphincter relaxation following bladder catheter pulling (Figure 30B). The secondary muscle spindle afferent fiber SP2(1) increased its activity in an undulating manner (Figure 30Ba). The parasympathetic fusimotors, driving the muscle spindle, innervated by the SP2(1) fiber, probably were not continuously active as suggested by Figure 30Bb, in contrast to the parasympathetic activity observed in HT6 (Figure 30Ab). The other secondary muscle spindle afferent fiber in paraplegic 9 (SP2(2), Figure 30Ba) slowly reduced its activity upon bladder catheter pulling. This spindle afferent fiber was not connected to the continence of the bladder. It is likely that its spindle was not parasympathetically innervated and was sited in leg muscles or parts of the pelvic floor muscles not contributing to continence. The $\alpha_{2}$ and $\alpha_{3}$-motoneurons (Figure 30Bc) showed a high activation, which is expressed in their oscillatory firing, and probably contributed to the continence of the bladder and the rectum. These likely sphincter motoneurons did not reduce their activity following parasympathetic activation, as can be seen from the SP2(1) fiber activity (monitoring parasympathetic activity). These motoneurons were not inhibited and the external sphincters were probably not relaxed. The somatic fusimotor $\gamma_{1}$ (Figure 30Bc) increased its activity transiently and slightly upon painful bladder catheter pulling, in similarity to a $\gamma_{1}$ fiber in HT6 [33]. The measurements in paraplegic 9 indicate a loss of the inhibitory action of the detrusor onto the sphincter motoneurons.

The time constant for the activity decrease of a spindle afferent fiber following parasympathetic activation was 31s in a paraplegic and approx. 40s in a brain-dead human (Chapter V of [17]). It is concluded that the muscle spindles are unchanged following SCI. The pathologic firing patterns of the SP2 fibers are thus probably a result of neuronal network changes in the parasympathetic and somatic nervous system divisions of the sacral micturition center.

In conclusion, in a brain-dead human (HT6) the sphincter motoneurons sub-serving continence were inhibited at a time, when preganglionic parasympathetic efferents increased their activity (physiologic) for 10s and an SP2 fiber increased its activity for several minutes. In a paraplegic with a strong bladder dysfunction, the SP2 fiber activity increased, due to parasympathetic activation, lasted for approx. one minute, showed undulations, and its amplitude was smaller than that measured in a brain-dead human. The sphincter motoneurons were not inhibited (pathologic).

By comparing the detrusor pressure curve and the sEMG of the pelvic floor of Figure 25B with the single-neuron recordings of Figure 30B, it can be seen that both diagnostics gave the same result, namely that the parasympathetic activation of the detrusor was undulating and not strong continuously and that the external bladder sphincter was not inhibited when the detrusor was activated. Therefore, the clinical urodynamics is a good clinical diagnostic for judging urinary bladder functioning. But the measurements at the single-neuron level gave more information, namely that the neural networks of the sacral micturition center in cooperation with the pontine micturition center were strongly dysfunction, including the impairment of phase and frequency coordination for neural network self-organization. But neural networks 


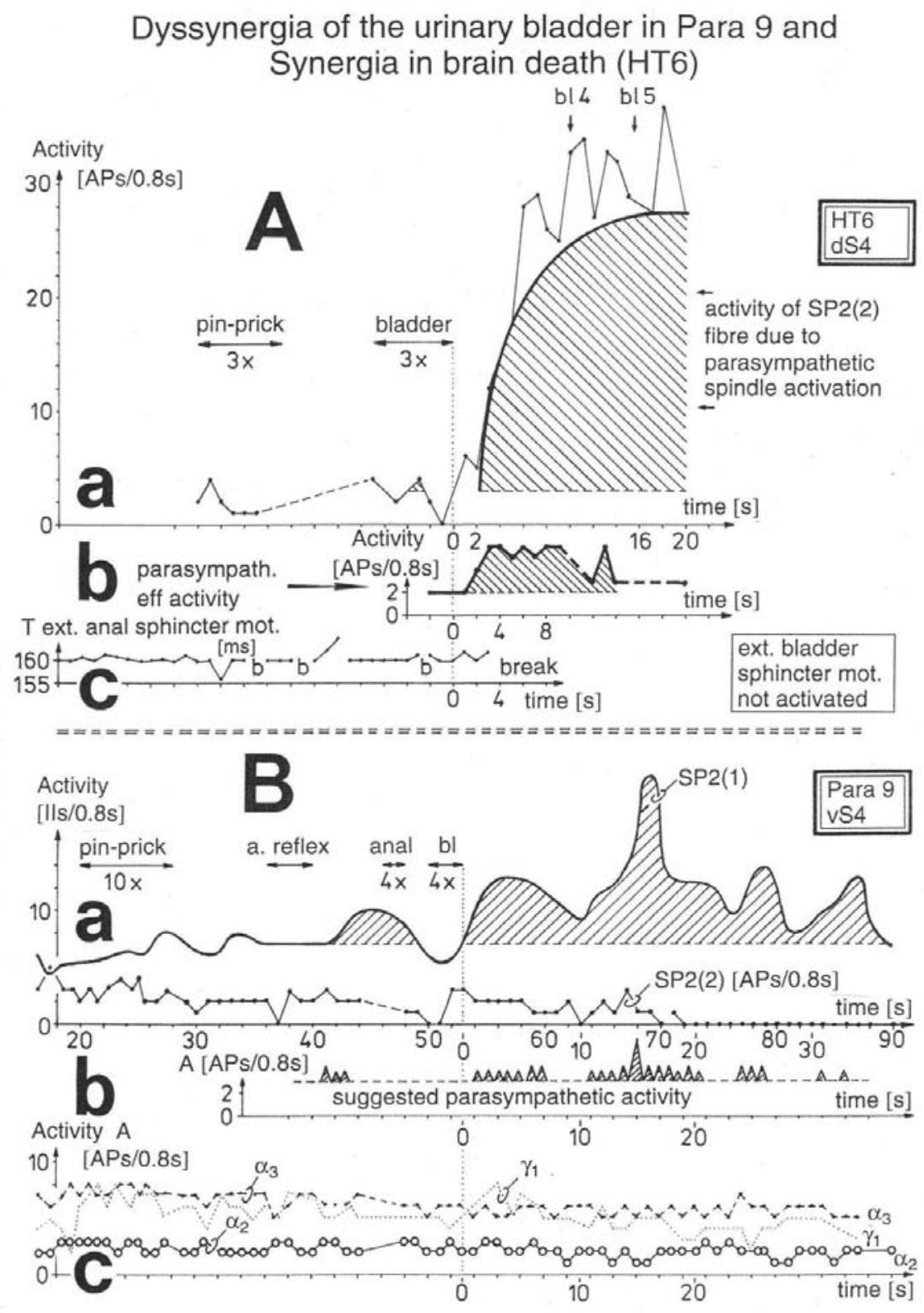

Figure 30: Direct comparison of secondary muscle spindle afferent activity and motoneuron activity between the brain-dead human HT6 with a synergy of the bladder (A) and the paraplegic 9 with a dyssynergia of the bladder (B).

A. Simultaneous measurements of activities of secondary muscle spindle afferents (a), parasympathetic preganglionic motoneurons (b) and oscillatory firing (high activity mode) of a sphincter motoneuron innervating the striated anal sphincter (c). Note that with the transient activity increase of the parasympathetic fibers (b) the secondary muscle spindle afferent fiber increased strongly its activity (a) for minutes, and the oscillatory firing sphincter motoneuron discontinued its oscillation (c) to reduce strongly its activity. bladder $3 \mathrm{x}=3$ times bladder catheter pulling. Text. anal sphincter mot. = oscillation period of the sphincter $\alpha_{2}$-motoneuron innervating the anal sphincter. For further details, see Chapter V of [1].

B. Simultaneous measurements of activity of secondary muscle spindle afferent fibers SP2(1) and SP2(2) following anal (anal $4 \mathrm{x}$ ) and bladder catheter pulling ( $\mathrm{bl} 4 \mathrm{x}$ ) (a), and the activity changes of an $\alpha_{2}$-motoneuron (FR) and $\alpha_{3}$-motoneuron (S) and a dynamic fusimotor fiber $\left(\gamma_{1}\right)$ (c). Note that following bladder catheter pulling (and probably parasympathetic activity increase), the spindle afferent fiber SP2(1) (most likely contributing to continence) increased its activity in an undulating manner (a), whereas the SP2(2) fiber did not (probably not connected to continence) (a), and the $\alpha$-motoneurons did not reduce their activity (c). The dynamic fusimotor $\gamma_{1}$ transiently increased its activity similarly as in HT6 measurements. In similarity to 'Ab', the suggested parasympathetic activity increase is pictured (b). a. reflex $=$ anal reflex stimulation. IIs = interspike intervals; IIs/0.8s $=($ APS 1) $/ 0.8 \mathrm{~s}$ (the activity measures IIs/0.8s and APs/0.8s differ by ' 1 '). 
can be repaired through CDT, which means urinary bladder functions can be repair by movement-based learning, as will be shown below. For further details of the dysfunction of the neural networks of the sacral micturition center see Chapter V of [17] and a review of coordination dynamics therapy [3].

3.1.13. Urinary bladder repair in $\mathbf{9 5 \%}$ spinal cord injury : A 17 -year-old female patient (Kadri) suffered a severe cervical SCI in a car accident. No motor functions remained below the injury level of C5/6 and the patient had impaired feelings. From the MR images the author estimated that approximately 5\% of the spinal cord matter was spared (Figure 19B,C). When the spinal shock faded away, it became obvious that no motor functions remained below the injury level but spot wise sensitivity remained more or less all over the lower body. Two months after the accident CDT was started. Upon 2.5 years of CDT the sensitivity improved and some motor functions returned below the injury level, indicating that some regeneration of the spinal cord had occurred. Urinary bladder functions were repaired. Details of the repair are given below (Figure 31). The connectivity over the injury site, according to the magnetic resonance imaging (MRI), may have increased to $8 \%$.

Generally, a urinary bladder repair is very important in rather complete C5/6 SCI. The tetras are not able to perform intermittent bladder catheterization by themselves, because of the mainly lost finger functions due to the lost finger-function-motoneurons in C5/6 spinal cord segments. Through urinary bladder repair, the patients get their private sphere back.

3.1.14. Transient increases of the coordination dynamics values fall together with the recovery of motor and urinary bladder functions: The changes of the coordination dynamics values of Kadri are shown in Figure 24 for 2.5 years of therapy. Transient worsening of the coordination dynamics (higher values) fall together with the improvement of motor functions and probably indicate successful regeneration of small amounts of nerve fibres. Following the largest peak of transient increase of the coordination dynamics values (indicating the strongest transient regeneration), the urinary bladder started to function rather physiologically again through 2.5 years of CDT.

3.1.15. The time course of the improvement of urinary bladder functions upon CDT: It is reported about the stages of bladder repair through 2 years of therapy. The changes of functions of the detrusor (bladder) and the external and internal bladder sphincters are extracted from a detailed anamnesis and are pictured by an evolution of the attractor layout (in similarity to motor function of Figure 15) with the re-learning of bladder functions (Figure 31).

1. After the operation (fixation and alignment of the broken cervical spine) a lying catheter was installed in the patient. The patient was suffering continuous infections and fever.

It is understood that the bacteria are 'creeping up' the lying catheter into the bladder (especially in female, because of the anatomy of the urethra) to give rise to ongoing infections in spite of antibiotic therapy. Before World War II (time of no antibiotics), patients were dying of such infections. It is the benefit of Sir Guttmann from Breslau [1] who stopped or reduced these infections by introducing sterile intermittent catheterization. The introduction of antibiotic therapy helped further.

2. One month later (at a time when the spinal shock weaned) a suprapubic catheter was installed and no more infections occurred. But the bladder did not show any physiologic functions. The patient had no feeling of bladder fullness, no desire to void and did not feel when the fluid was leaving the bladder. The catheter was used for emptying (when opened) and storing (when closed) the urine. Since no fluid was leaving the bladder through the sphincters, the external striated sphincter was spastic (continuous contraction) and the internal sphincter (smooth muscle), as a part of the detrusor, was probably not working.

Physiologically, the internal sphincter (smooth muscle, slowly acting, probably a part of the detrusor) is keeping the continence for low and medium bladder pressure. For high bladder pressure 
Development of attractor 'Urinary bladder function' upon Coordination dynamics therapy

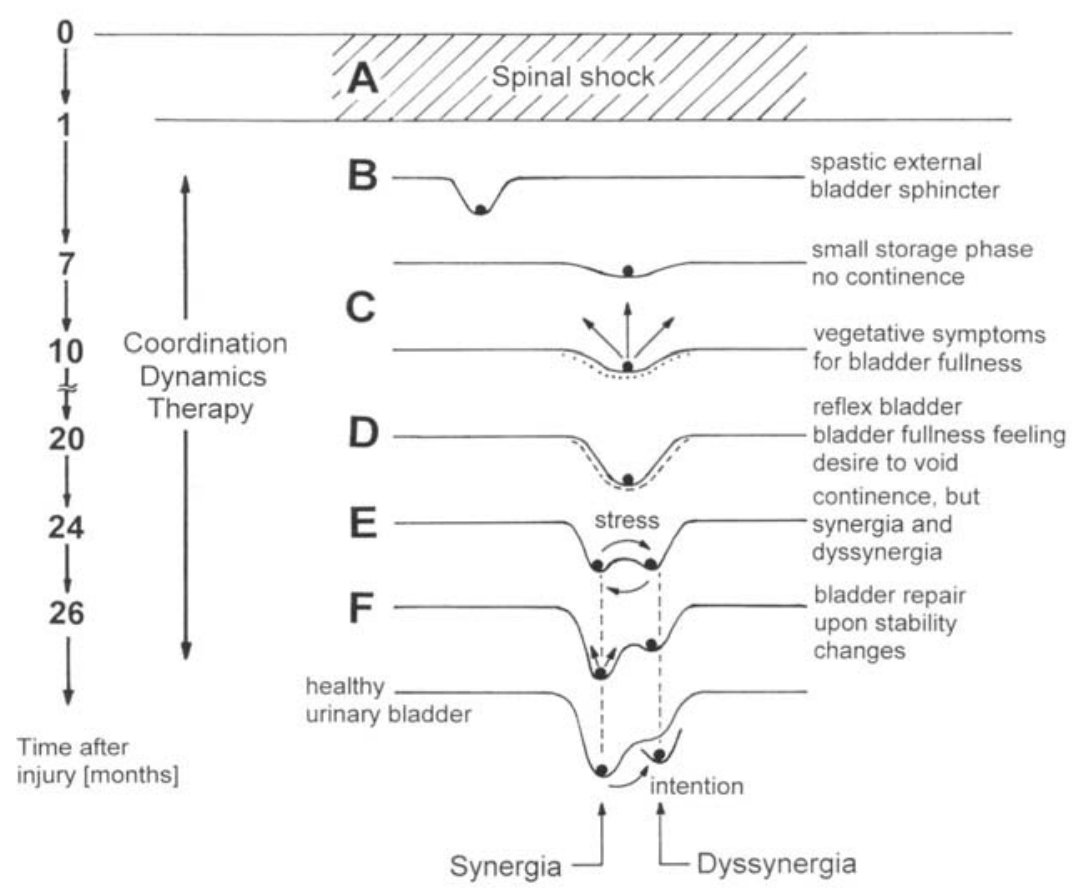

Figure 31: Evolution of the attractor layout of bladder functioning induced by learning transfer from movements to bladder functions upon CDT. The region around each local minimum of the potential landscape acts like a well that weekly traps the system into a coordinated state. Black balls correspond to stable minima of the potential. With learning, the pattern 'spasticity of the external bladder sphincter' vanishes and the patterns for bladder functioning ('synergy' and dyssynergia') appear anew and gain their physiologic stability (physiologic deepness of each basin of attraction). The corresponding attractor layout for physiologic bladder functioning is given. Fluctuation of pattern state (the black ball) (C), and their decrease (F), due to the impairment of phase and frequency coordination of neuron firing, is pictured in ' $\mathrm{C}$ ' and ' $\mathrm{F}$ ' by long and short arrows. Dotted and dashed lines indicate the re-occurrence of bladder sensation. Note that more than two years of optimal continuous CDT were needed for bladder repair.

and sudden bladder pressure increases (as for example during coughing), the rather fast-external sphincter (innervated by $\alpha_{2}$-motoneurons and consisting of fast fatigue resistant muscle fibers (FR), Figure 11) is contracting to secure continence. If the striated external sphincter does not work properly, patients suffer the so called 'stress incontinence'.

3. Seven to eight months after the accident (end of 2005), the fluid was leaving the bladder by itself after a small storage phase. This means that therapy had reduced the spasticity of the external sphincter. The patient was now inconvtinent. So far, the spastic external sphincter had mainly stopped the fluid from leaving the bladder by its spasticity. The internal sphincter started to work a bit to allow a small storage phase. When the fluid was leaving the bladder, there was first no feeling of fluid movement. Later on, the patient had some feeling of fluid movement. Probably flow receptor afferents (S2) (Figure 27) started to work. Three months later the suprapubic catheter was removed. The patient started to use diapers.

4. 20 months after the accident (beginning of 2007, upon 18 months of therapy) the patient felt bladder fullness and the desire to void. Probably stretch (S1) and tension receptor connections (ST) (Figure 27) started to work again. The vegetative symptoms of bladder fullness information (sweating and sudden heard rate increases, probably transmitted by plexus connections) were replaced by bladder fullness feeling and the desire to void.

5. The patient became able to press the fluid out of the bladder. To get all fluid out, the reflex bladder 
had to be activated a bit, by tapping, touching or massaging the skin above the urinary bladder, which is the reflex skin area for the bladder reflex (Zones of Head, Figure 64). Sometimes body positioning was used to influence the bladder reflex. With these maneuvers the desire to void reappeared and the patient could empty the bladder further.

Often patients (to whom no CDT is administered) are training the bladder reflex for emptying. The reflex is stimulated by tapping the skin above the bladder. But if, for example, the external sphincter is spastic (as in this patient), it may not be possible to generate a good functioning reflex bladder. The neural networks of the sacral micturition center are working too pathologic.

6. After the appearance of the desire to void, the patient became able to hold the fluid for 30 s till $1 \mathrm{~min}$. Sometimes she could keep the continence better and sometimes not so good. This means that the external bladder sphincter (which can be controlled volitionally) started slowly to work, but irregularly. The feeling of bladder emptying became similar to those before the SCI.

7. 24 months after the accident (spring 2007), the bladder started to function rather physiologically again. After a storage phase the fluid came out on volition. The detrusor started to work fully. But if the patient was pressing too much at the end of bladder emptying to get all fluid out (to reduce the residual urine, not to get bladder infections), the external sphincter contracted. The external sphincter co-contracted with the detrusor. Detrusor-sphincteric dyssynergia of the urinary bladder occurred. But when she then activated the reflex bladder by tapping or touching the reflex bladder area, the desire to void reappeared and she could empty the bladder fully. The residual urine was not measured.

At this stage of bladder repair two patterns existed: the synergy of the bladder, in which the detrusor and external sphincter contracted antagonistically, and the dyssynergia of the bladder, in which the detrusor and the external sphincter co-contracted (Figure 25B). The synergy pattern was for emptying the bladder and the dyssynergia pattern was the pathologic pattern. The pathologic co-contraction of the external sphincter with the detrusor occurred more easily when there was less fluid in the bladder and the patient had to press more (inducing stress to the CNS).

Physiologically both bladder emptying patterns do exist. The synergy pattern is for emptying the bladder and the dyssynergia function is for stopping the micturition. But both patterns are under volitional control.

8. Upon two years of CDT (26 months after the accident) the patient was full continent again and could empty the bladder on volition. The time interval from the first feeling of the desire to void to the situation that the fluid was coming out by itself (including 4 times of occurrence of the desire to void) was one hour. The patient did not use diapers any more. The patient had never used drugs which are supposed to improve urinary bladder functions. The repair of urinary bladder functions was achieved by the re-learning of urinary bladder functions including transfer of learning from the movements jumping on springboard, treadmill walking (Figure 19EF) and exercising on the special CDT device. The strong improvement of urinary bladder functions occurred, when the coordination dynamics values strongly increased (got worse) (Figure 24), indicating a bit of regeneration.

For patients with incomplete spinal cord injuries, it is very important how long they can hold the urine from the first desire to void till to the moment when the fluid comes spontaneously. Can they safely reach the WC or not? The improvement of bladder functioning can also be judged by how long the patient can hold the fluid following the first desire to void. In this case it was 1 hour after 2 years of CDT.

The feeling of the lower abdomen, which was poor after the accident, improved also strongly at the time of nearly full bladder repair. The patient felt again the lower abdomen very good (inside and outside as the patient reported) and felt also again the working of the abdominal muscles. At that time, also the finger functions got a tiny bit better and her supported treadmill walking improved (Figure 23H). During walking on treadmill, and during other movements, the patient got goose-pimples all over the body. It seems that an 
overall improvement of vegetative and somatic functions occurred the time of full bladder repair.

3.1.16. Attractor layout changes during urinary bladder repair: Within the framework of System Theory of Pattern Formation, the repair of the urinary bladder functions can be understood and pictured by the changes in the attractor layout.

One month after the injury, when the spinal shock faded away (Figure 31A, only the pathologic bladder pattern 'spasticity of the external sphincter' was present (Figure 31B). Six to 10 months later, the spasticity of the external sphincter reduced and a small storage phase of the bladder re-appeared as a first sign of bladder repair (Figure 31C). 20 months after the operation, the reflex bladder pattern organized itself with bladder fullness feeling and the desire to void (Figure 31D). 24 months after the accident, the attractor layout showed two attractors, the bladder synergy (the detrusor action inhibits the external sphincter) and the dyssynergia (co-contraction of detrusor and external sphincter) (Figure 31E). The state of the system (pictured by the ball) switched easily from the attractor synergy to the attractor dyssynergia. 26 months after the accident, the stability of the attractor synergy had increased and the stability of the attractor dyssynergia had decreased (Figure 31F). On volition (intention), the micturition could be induced and stopped as in healthy individuals.

3.1.17. Conclusion: Upon 2.5 years of CDT urinary bladder functions could be cured in severe (95\%) cervical SCI. Since the injury was motoric complete and the cord was 'free' in the spinal canal (Figure 19BC, the cord does not touch the vertebrae), some regeneration in the human spinal cord should have occurred.

3.1.18. Integrative functions and central pattern generators for urinary bladder repair: It was shown in this patient Kadri with a 95\% SCI that urinary bladder functions could be cured upon 2.5 years of CDT. Three important steps were achieved. First, the patient got the bladder under volitional control again. Second, a physiologic attractor layout for bladder patterns could be generated; and third, the dyssynergia of the urinary bladder could be cured by increasing the stability of the synergy pattern and decreasing the stability of the dyssynergia pattern. The stability changes of the two bladder functioning patterns can be understood within the framework of system theory of pattern formation and human neurophysiology [1719] but not in the framework of central pattern generators (CPG's). This knowledge is used below for the bladder repair of the 9-year-old Nefeli with an SCI of approximately 70\% at the level of Th10. In Nefeli a transient regeneration of the spinal cord could be achieved and quantified by segment-indicating muscles (Figure 37).

3.1.19. A spinal cord injury up to $\mathbf{8 0 \%}$ may still allow the re-learning of walking : The repair of a SCI depends on the severance of the injury, the efficiency of the repair treatment and how aggressive the therapy is applied. In Figure 19 it was shown that in a patient with a 50\% SCI, the urinary bladder function was fully repaired via 2 months of CDT and the patient Sten could re-learn walking and running through 2 years of CDT. In the patient Kadri with a 95\% SCI, 2.5 years of CDT were needed for the bladder repair and the walking could not be achieved. The question is now, up to what severance of injury a re-learning of walking is possible through an efficient, aggressively and continuously applied treatment. The believe is that up to an injury of $80 \%$ a re-learning of waking is possible. The repair of the bladder is anyway possible though a long-lasting CDT. Plexus connections and the sympathetic chain (Figure 26) are probably contributing to get the lower body partly under control again.

Below it will be shown that the patient Nefeli with an approximately $70 \%$ SCI ( $30 \%$ of the cord remained) could re-learn walking. The importance of this case report and the upper considerations is that most spinal cord injuries are incomplete and substantial repair is possible.

\subsection{Regeneration of the human spinal cord during development}

3.2.1. Summary of the repair of a $70 \%$ spinal cord injury at the level of Th10 (10-year-old girl Nefeli): In the 5.5-years-old Nefeli a neuroblastoma was found to grow from the Th10 ganglion. With the surgery to remove the cancer she suffered a SCI at Th10/11 levels by medical malpractice. An 8-months- 
rehabilitation in Switzerland brought only little progress. Most of the repair was probably due to spontaneous recovery. When Nefeli started school, she was incontinent and could not walk. An assistance helped her to manage at school (Figure 32B).

At an age of 9 Nefeli started CDT with the Author. Spasmolytic drug and urinary bladder medications were stopped. Following four weeks of aggressive CDT, supported walking and jumping improved. The crawling became better. Following three years of CDT, Nefeli became mainly continent and she learned to walk (Figure 32) with some balance problems. She learned to creep, crawl and jump. Even a bit of running became possible. At an age of 12.5 years, following 3.5 years of CDT, Nefeli learned to ride a normal twowheel bicycle.

3.2.2. Upgrading of coordination dynamics therapy: Above it was stated that CDT consists of the
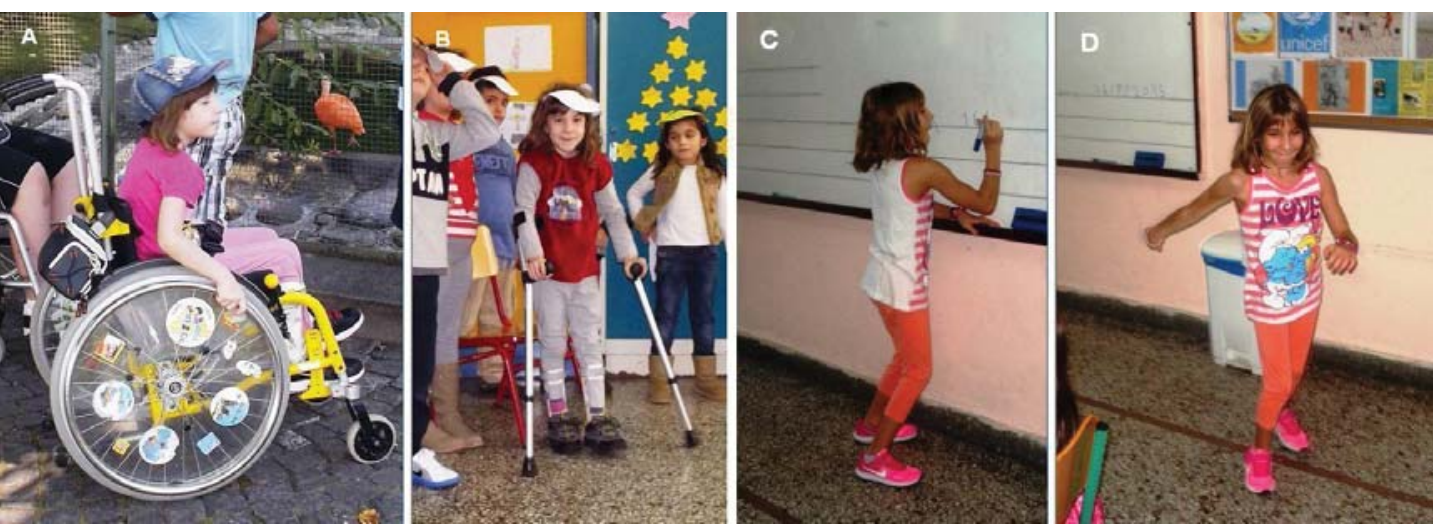

Figure 32: A. The 5.5-year-old Nefeli after suffering an incomplete SCI Th10 by medical malpractice. B. Nefeli after eight months of conventional children rehabilitation in Switzerland (Affoltern). Sticks and orthosis were needed. C,D. Ten-year-old patient Nefeli after six months of CDT. At school she can walk again and can write at the white board.

improvement of phase and frequency coordination for an improved neural network organization and the re-learning of movements like creeping (Figure 33AB), crawling, walking, running, jumping and other to the patient adapted movements so that other brain parts can take function over from the injured ones by plasticity. The right-left communication of the hemispheres via the corpus callosum (Figure 33D) can be improved, which is especially important in stroke patients, by especially exercising on the special CDT device with crossed arms (Figure 33C).

In the patient Nefeli additional trained movements were the phylogenetic old movements like pushing forward movements as Tiktaalik's may have used 375 million years ago (Figure 34) and Salamander crawling (Figure 35). Hula-hoop for improving trunk movements and stability and reduce scoliosis was also used (Figure 81).

3.2.3. Revised treatment strategy to enhance spinal cord regeneration: The first 10 months of therapy, the strategy of Nefeli's CNS repair was mainly to use optimally what was spared following the SCI. This means activating and re-wiring the remained tract fibers at the injury site with the help of plasticity of the brain to use the spared fibers for all the necessary functions below the injury level. But when it turned out that her damaged spinal cord partly regenerated, the CNS repair had to be seen in a more general way. Now it had to be considered additionally what performed movements and other patterns enhance the regeneration of nerve fibers in the spinal cord. It had to be clarified at what site the regeneration was mainly impeded and how can nerve fiber grows induced and guided and false growing (mismatch) reduced. The contribution from stem/progenitor cells to the regeneration had to be considered. 

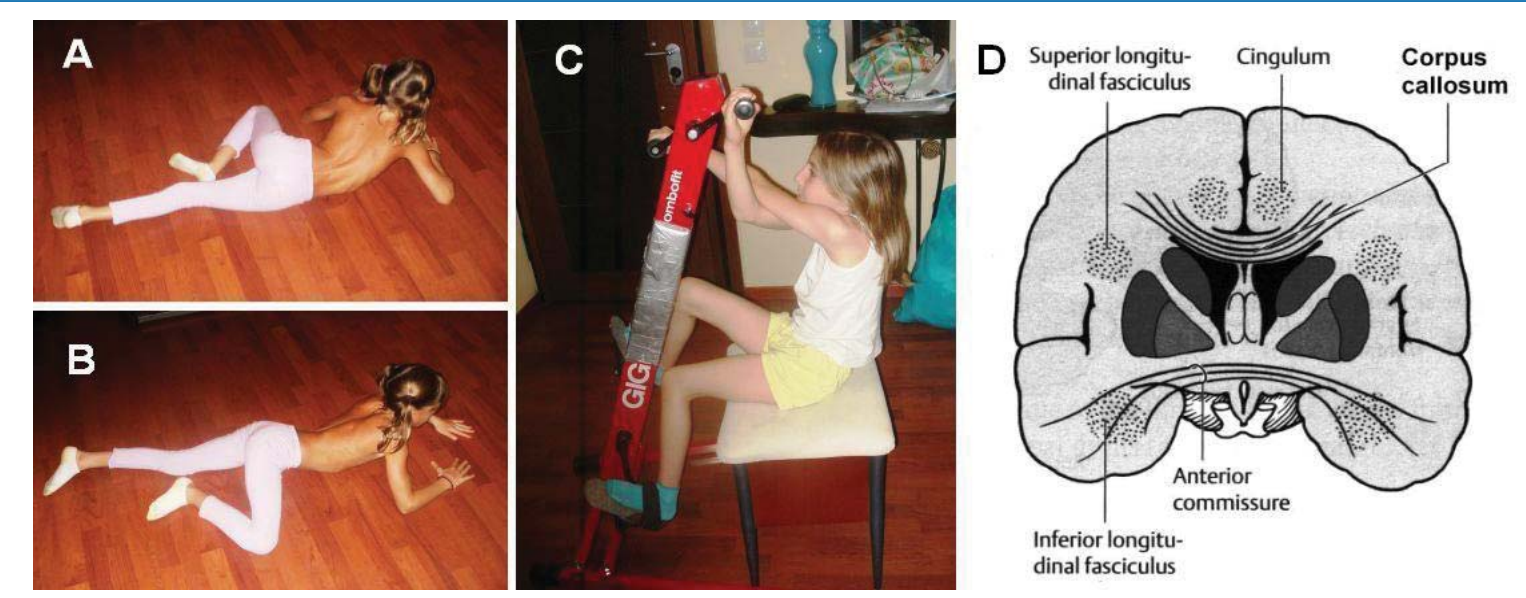

Figure 33: A, B. Ten-year-old patient with an incomplete SCI at the Th10 level during creeping. Note, because of the severe scoliosis, the creeping is not symmetrical. The rotation to the left is limited because of the scoliosis. C. Training of right-left symmetry via the corpus callosum (D) when exercising on the special CDT device with crossed arms.
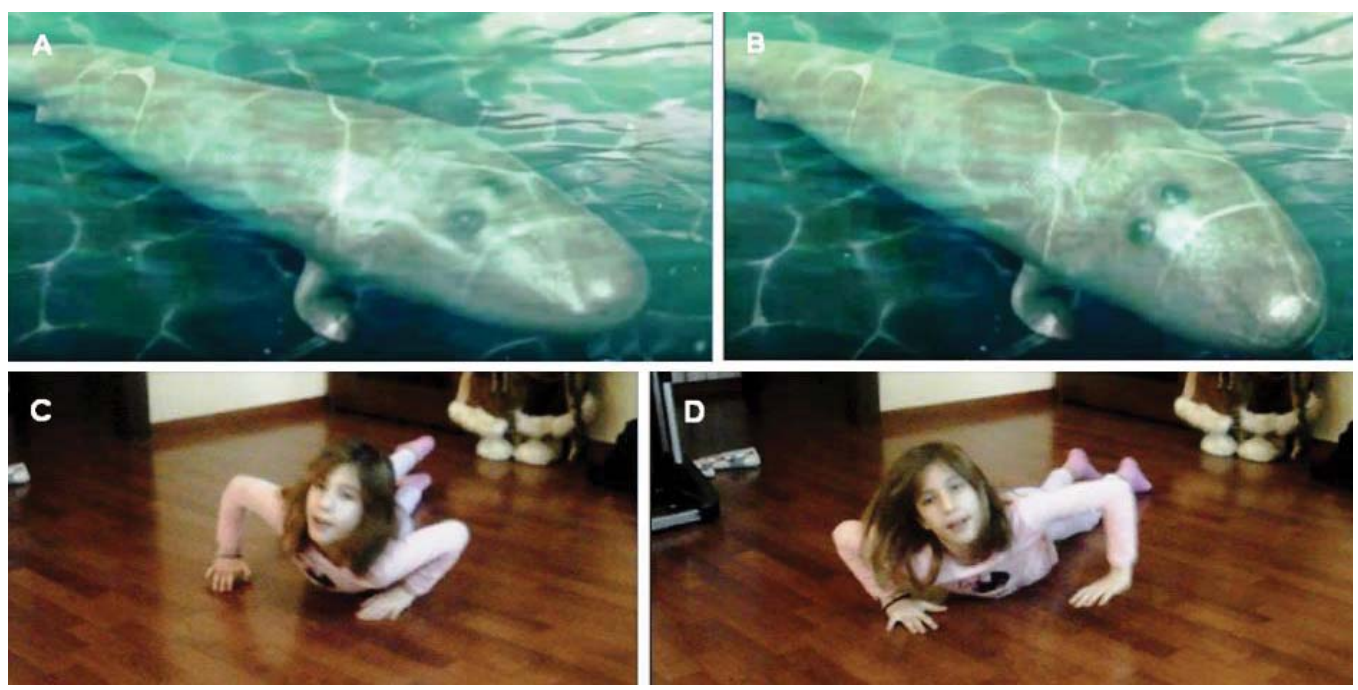

Figure 34: A, B. Possible rotational body movement of Tiktaalik, caused by alternately using one front limb for forward locomotion. C, D. This front limb movement is simulated by a patient with a SCI (Nefeli) by using alternately the right and left arm. Tiktaalik roseae is a lobe-finned fish from the late Devonian period, about 375 million years ago, having may be features akin to those of four-legged animals (tetrapods). Tiktaalik has a possibility of being a representative of the evolutionary transition from fish to amphibians.
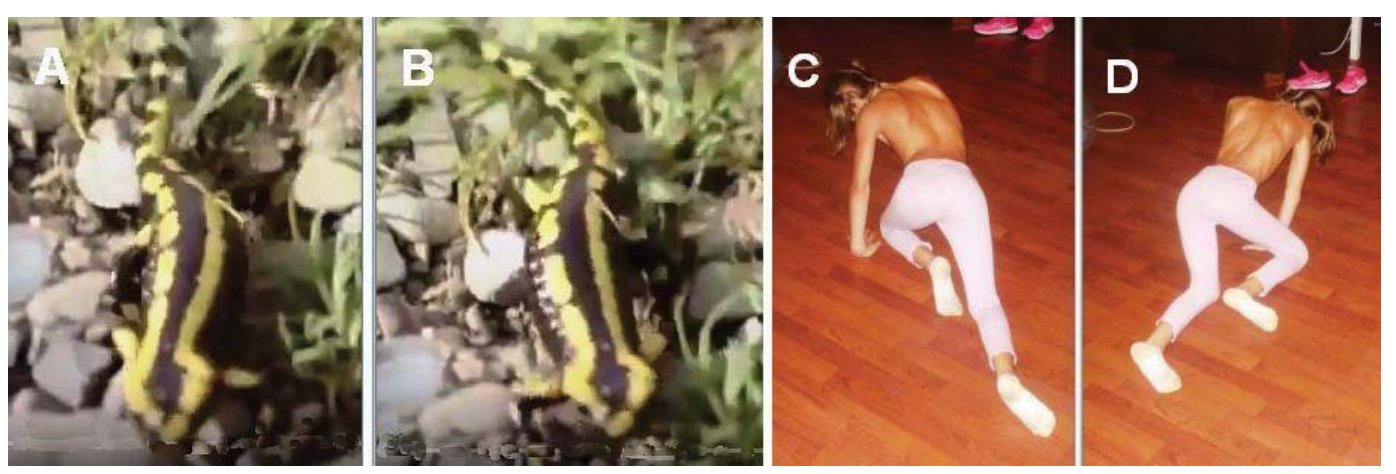

Figure 35: Salamander-crawling (C, D) in similarity salamander-walking (A, B) of the 10-year-old Nefeli with a SCI (Th10). In D the bending is disturbed because of the scoliosis and spasticity. 
The improvements of motor and bladder functions during the first six months were probably mainly due to a functional repair (functional reorganization in the brain) of the spared tract fibers 4 years after the injury. But after 10 months of therapy there was indication that motor functions of the legs were reappearing, caused by a regeneration of the spinal cord. To clinically follow up the regeneration of Nefeli's spinal cord, the human anatomy, including the functional anatomy [34-36], had to be taken into account to see how the regeneration was taking place and how the therapy could be further optimized. The speed of regeneration will be measured below and it will be considered what has to be done to enhance regeneration.

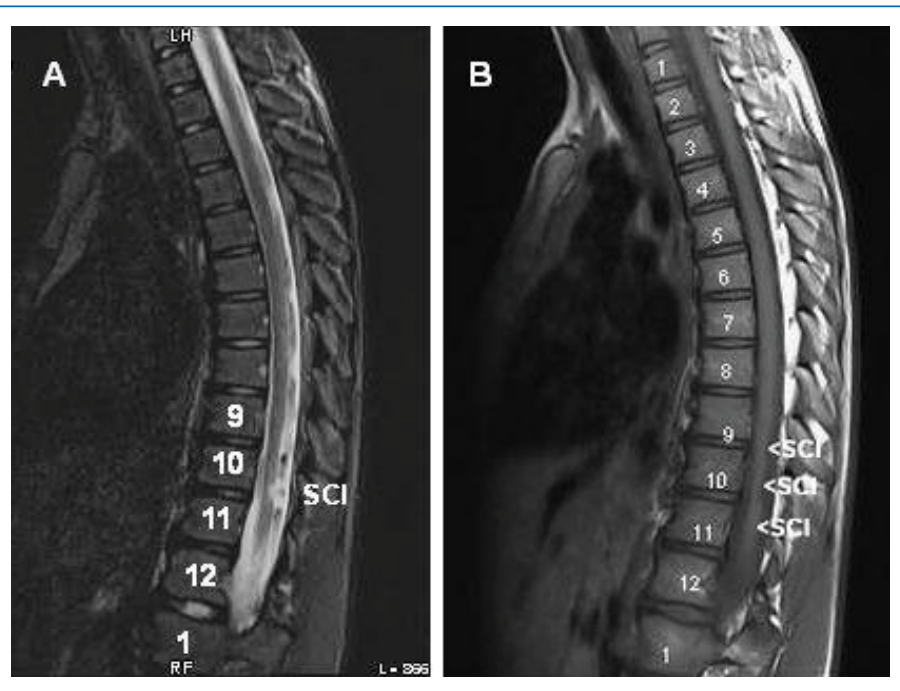

Figure 36: MRI of the 10-year-old patient Nefeli with a SCI at the levels around Th10. Because of scoliosis, the spinal cord is not fully in the plane. The injury of the spinal cord is mainly from the dorsal site.
Nefeli suffered an incomplete SCI at the level of Th10/11 (Figure 36). Approximately $30 \%$ of the spinal matter was spared. Figures 1 and 2 show the spinal cord with the spinal segments and the corresponding dermatomes of the body. To identify the re-innervated cord level and calculate the speed of cord regeneration, segmentindicating muscles should be activated on volition again in the cephalo-caudal direction. Sensory innervation changes may not be characteristic for the identification of regenerated cord levels because Nefeli had an incomplete SCI and the remained sensory distributions were complex. Still sensory repair is as important as motor repair. Especially in the lower sacral range there is a large overlap and variation of the dermatomes [37]. The segment-indicating muscles seemed to be suitable for identifying the level of the re-innervation for certain tract fibers. A muscle starts to contract when sufficient motor units are activated to counteract movement resistance.

In the peripheral nervous system (PNS) of the frog the thickest myelinated nerve fibers regenerated fastest [17]. In human it will probably be the same. Figure 9 shows the conduction velocities and nerve fiber diameters of human nerve fibers of the PNS. Classification schemes for the human tract nerve fibers in the spinal cord do not exist. It is therefore assumed that there are similarities between the human CNS and PNS. Further it is not clear whether tract neurons regenerate and/or relay neurons are built at the injury site or even new tract neurons are built anew from stem cells in the sensory-motor cortex. Newly built neurons are most suitable for repair. Also, mismatch has to be considered. Neuron grows along wrong pathways, which has to be compensated for by brain plasticity. Occurring mismatch has to be reduced. The repair and regeneration mechanisms are very complex. Still it is astonishing that some reasonable results will be obtained, when analyzing the speed of regeneration in Nefeli's spinal cord.

3.2.4. Speed of regeneration of the human spinal cord in children: Figure 37 shows the segmentindicating muscles and partly cutaneous sensory distributions of the L4, L5, S1, S2/3 and S4/5 nerve roots or spinal cord segments.

It was measured in Nefeli at what times the different segment-indicating muscles started to work again in cephalo-caudal direction. At the second of February Nefeli was able to lift substantially the knees and dorsal flex a bit the feet (Figure 60) but could not activate the big toes separately or abduct the small toes. The quadriceps femoris and the tibialis anterior could therefore be activated again. A part of spinal cord regeneration had reached the L4 level (Figure 37). At the 12th of February she could activate the extensor halluces longus muscle (Figure 38), which activates the big toe for dorsal flexion and represents the L5 spinal segment. At the 18th of February she could first time in the morning after sleep abduct and adduct the 


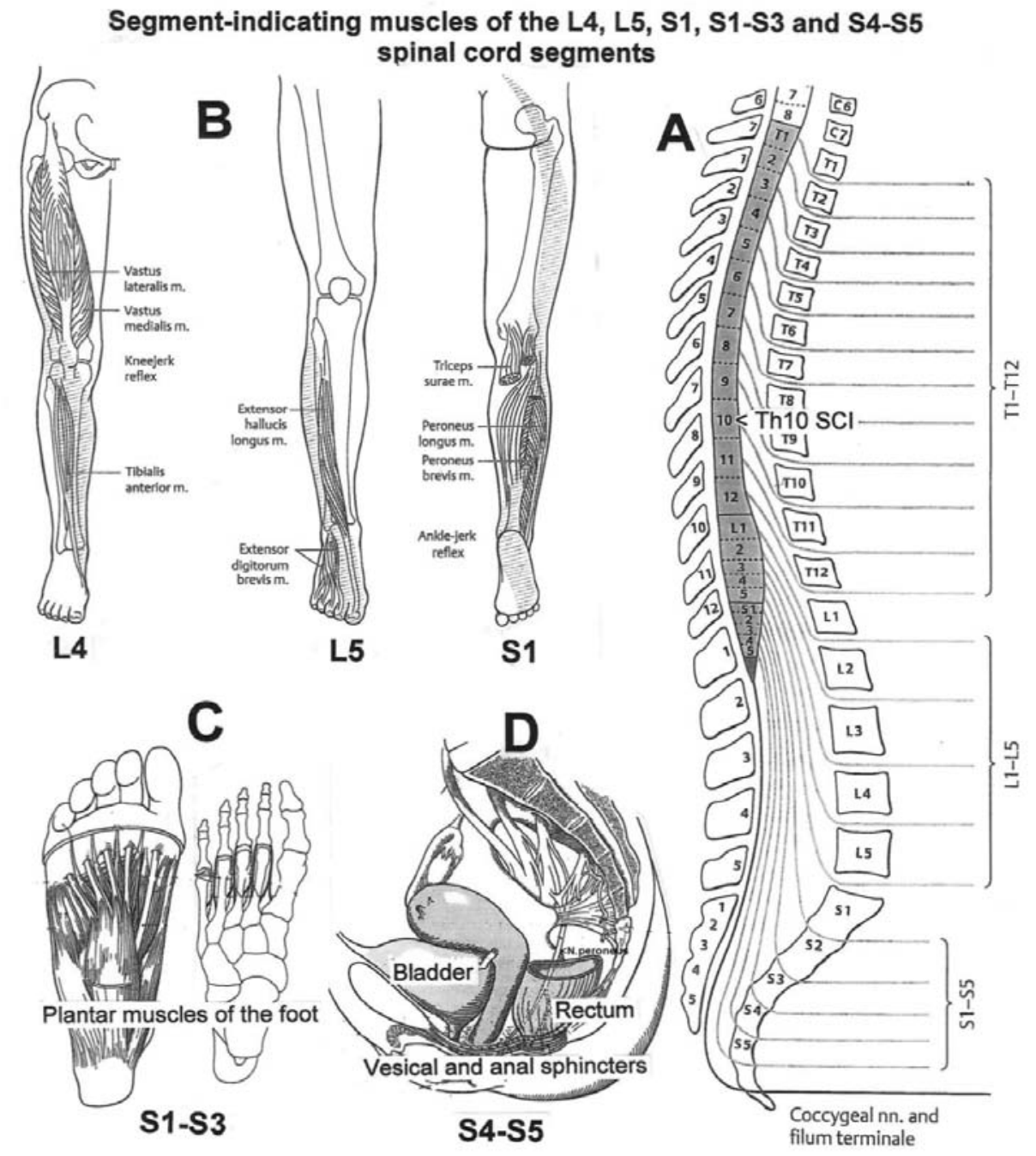

Figure 37: Segment-indicating muscles of the L4, L5, S1, S2/S3 and S4/S5 spinal cord segments for measuring the level of spinal cord regeneration. A. Relation between spinal cord and vertebra segments. B. The spinal cord segment L4 indicating muscle is for example the quadriceps. The extensor halluces longus is characteristic for the L5 segment. C. The plantar muscles of the foot represent S1 to S3 spinal cord segments. D. The vesical and anal sphincters are activated from the S4/S5 spinal cord segments. The skeletal muscles of the leg are innervated by $\alpha_{1}, \alpha_{2}$ and $\alpha_{3}$-motoneurons, but the external bladder and anal sphincters are innervated only by $\alpha_{2}$-motoneurons.
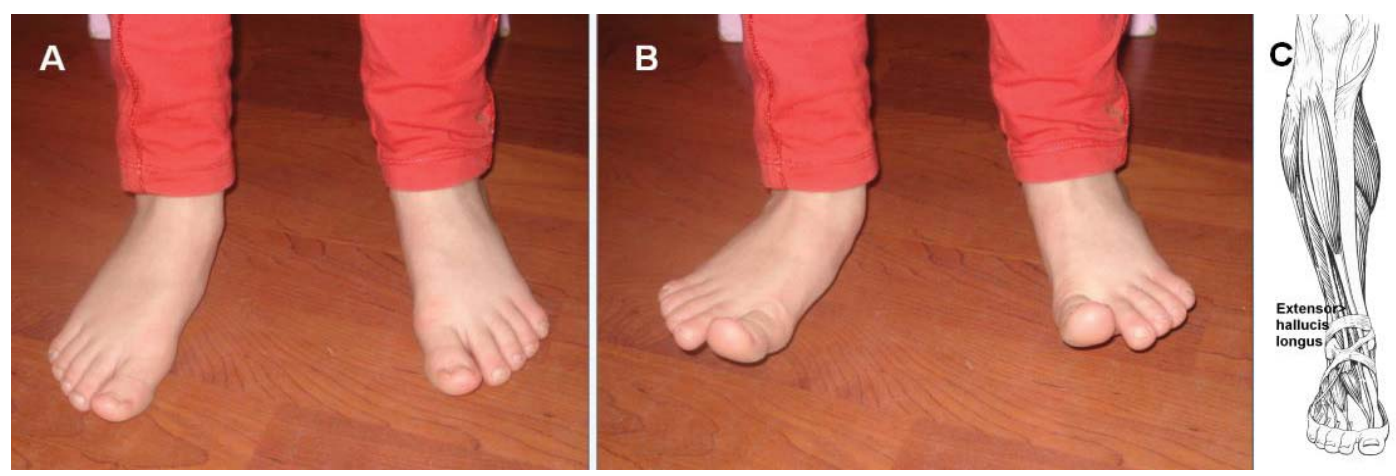

Figure 38: Activation of the halluces longus muscle (C) when dorsal flexing the big toes separately (B). Spinal cord regeneration reached the L5 level. 
small toes (Figure 39) which represent the S1-S3 segments. The fastest regenerating fibers had reached the motoneurons of the S1-S3 segments for muscle activation. The external bladder and anal sphincters (S4/ S5) could not be activated substantially so far. The sphincter muscles (somatic) are not innervated by fast conducting *1-motoneurons, but only by the thinner and probably slower regenerating *2-motoneurons (Figure 9).

The distance between the rootlets vL4 and vS2 (Figure 3B) is $20 \mathrm{~mm}$ in adults. The growing of the nerve fibers from the segment vL4 to vS2 spinal segments needed 13 days. The time for building functional synapses are the same for all muscles indicating segments. But Nefeli is smaller $(130 \mathrm{~cm})$ than adults, the cord distance is reduced therefore to $20 \mathrm{~mm} \bullet 130 / 180=14 \mathrm{~mm}$. The regeneration needed 13 days for the $14 \mathrm{~mm}$ that means approximately $1 \mathrm{~mm}$ /day. In the peripheral nervous system, the fastest regenerating fibers need 1 to $2 \mathrm{~mm}$ per day for regeneration [17].

Now the time is calculated which the nerve fibers probably needed to grow down from the injury site Th10 to the S2 segment. The cord distance from vTh10 to vS2 (Figure 3B) is approximately 90mm • 130/180 $=65 \mathrm{~mm}$. With $1 \mathrm{~mm} /$ day regeneration speed we obtain 65 days for growing. Assumed that the building of functional synapses needed approximately 35 days, the regeneration down the spinal cord till to the foot muscles and the building of functional synapses needed 100 days. The administered CDT time was ten and a half months that means approximately 320 days. Therefore, before the regenerating fibers grew down the human spinal cord, 220 days were needed. That means, approximately two thirds of the regeneration time were needed to start the regeneration in the spinal cord below the injury level.

Assumed that tract fibers regenerated from above the injury site, the time needed for crossing the 2 to $10 \mathrm{~mm}$ large gap (Figure 36) was 200 days, which is quite a long time. In rat the longest observed growing distances are a few $\mathrm{mm}$ up to $20 \mathrm{~mm}$. In human we expect much shorter possible growing distances, because in the PNS in nerve sutures the nerves have to be adapted for enabling nerve fibers grows into the distal nerve part. A substantial regeneration of tract fibers is therefore unlikely.

If we assume that relay neurons were built from stem cells at the injury site and neural networks were built in the gap from which the spinal cord regeneration started, then this neural network building process needed 200 days. But where are the neural network stem/progenitor cells are migrated from? Are some stem cells in the spinal cord or did they migrated from other places or were they transported in blood vessels?

A neural network building is likely, because Nefeli could contract the muscles only slowly. Further,
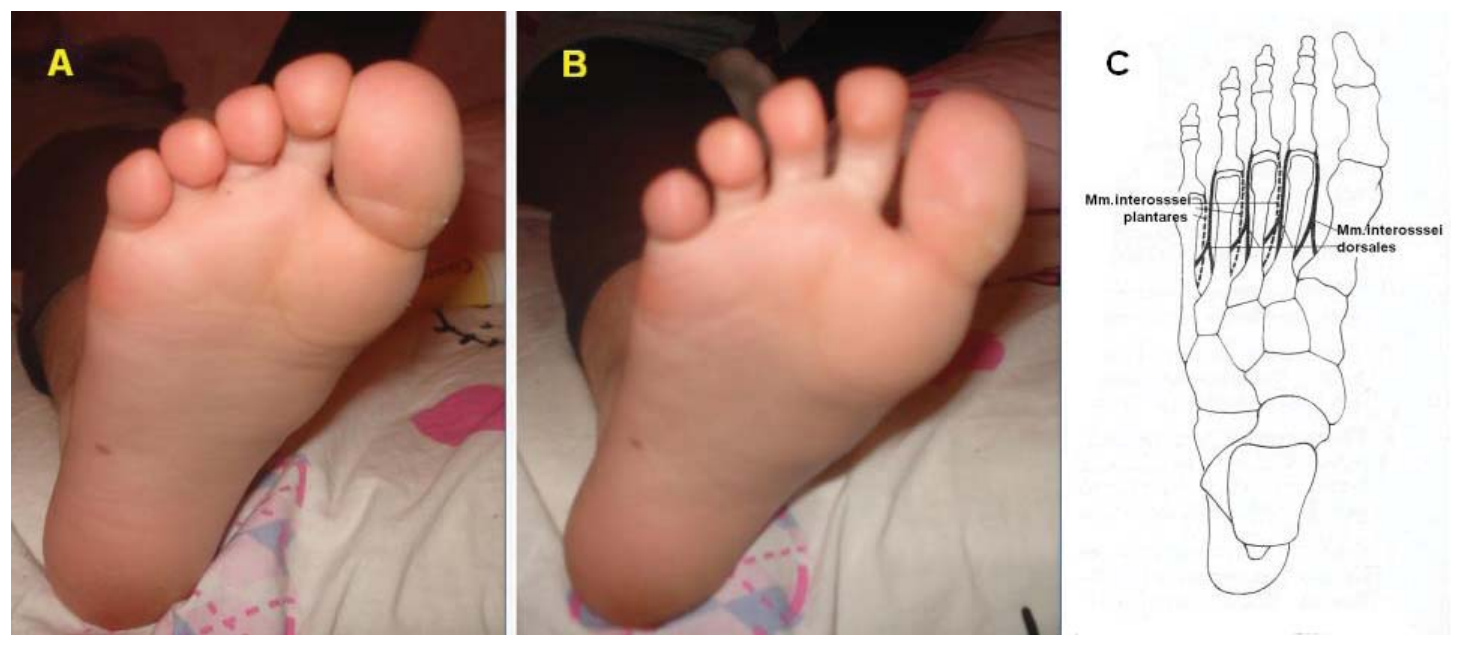

Figure 39: A-C. Volitional abduction and adduction of the toes becomes possible again in the SCI patient Nefeli. With the activation of the $\mathrm{Mm}$. interrossei dorsales and plantares (C), the regeneration of the spinal cord has reached the S1 to S3 spinal cord segments. 
the building of neural networks for spinal cord re-connection will take more time if the gap is larger. By comparing the SCI site of Nefeli (Figure 36 left) with that of the 17-year-old patient Kadri with a cervical SCI, it can be seen that Kadri's SCI gap was much larger (longer) (Figures 19B,C) and a bit of regeneration in the cephalo-caudal direction needed three to five years.

To understand the cause of repair in humans is of importance because it may have consequences for the treatment to be administered. If neural networks are built at the SCI injury site, then the building of these networks has to be enhanced by training those movements of which the activated neurons lie in and around the injury site. To activate and stimulate neural network growing at the Th10/11 injury site, Nefeli was training with the hula hoop (Figures 72,81), trained trunk rotation on the special CDT device (Figure 56A,B) and trained several rotational movements of the Tiktaalik (Figure 34). Stem/progenitor cells do only proliferate if the membranes are depolarized [68]. That means, the neurons around the injury site have to be activated by certain movements. Further, the tract fibers for all functions below the injury level have to be activated to make them growing into the neural networks at the injury site and stimulate the relay neurons to grow into degenerated tract fibers below the injury site.

The regeneration considered is very complex and only the fastest growing fibers reaching caudal spinal cord segments were measured so far. Many more fibers and thinner/slower regenerating fibers have to grow down to the caudal spinal cord and bring more motoneurons (motor units) and interneurons under supra-spinal control to generate more functional muscle power.

The improvement of the sensory innervation has not been analyzed so far. To get more feeling in the soles of the feet for better walking and balance performance, sensory fibers, entering the lumbar-sacral spinal cord, have to regenerate in the caudal-cephalon direction across the injury site.

Conclusion, even though much more knowledge about the regeneration of the human spinal cord is needed, this documented regeneration of the human spinal cord is a history progress. It is the first time, documented with sufficient diagnostic, that the human spinal cord partly regenerated by movement-based learning. Through 320 days of therapy, nerve fibers grew from the Th10 spinal segment down to the S2 segment. With a growing velocity of $1 \mathrm{~mm} /$ day, the $65 \mathrm{~mm}$ of the cord were covered in 65 days. Assuming 35 days for the building of functional synapses, 100 days were needed for growing caudally. This means that that 220 days of the 320 were needed to start the regeneration process at the Th10 level. The critical area of the regeneration process is located at the injury site and needs special considerations with respect to treatment.

Also, the plasticity of the brain is strongly involved in SCI repair. Whatever neurons are built anew and fibers are growing caudally or rostrally in the cord, the brain has to make the neural network changes functionally correct.

3.2.5. Repair of sexual functions : In the sensory-motor cortex the toe functions are sited close to genital and bladder functions (Figure 40). The repair of toe functions may help therefore with the functional repair of bladder and sexual functions. Most spinal cord injuries occur in man between 20 and 25 years and their biggest problem is the disturbed sexual function even though the repair of the bladder functions is more important. The conventional management of the sexual function in SCI is given in [38,39]. But a causal therapy of the disturbed sexual function is the repair of the nervous system. The Author is telling the male patients with a SCI that if the urinary bladder functions are repaired by aggressive CDT, then also the caudal sexual function will improve because both functions are located in the S2 to S5 segments (Figure 26) and there is learning transfer from movements to vegetative functions including sexual functions. Also, in the sensory-motor cortex (Figures 40) the sexual and bladder functions are close to leg and foot functions, making a learning transfer from foot function repair to bladder and sexual function repair easier. With the improvement of leg, foot and toe functions by movement-based learning as for example jumping on springboard in antiphase, also the adjacent neural networks, that means sexual and bladder functions will improve. 


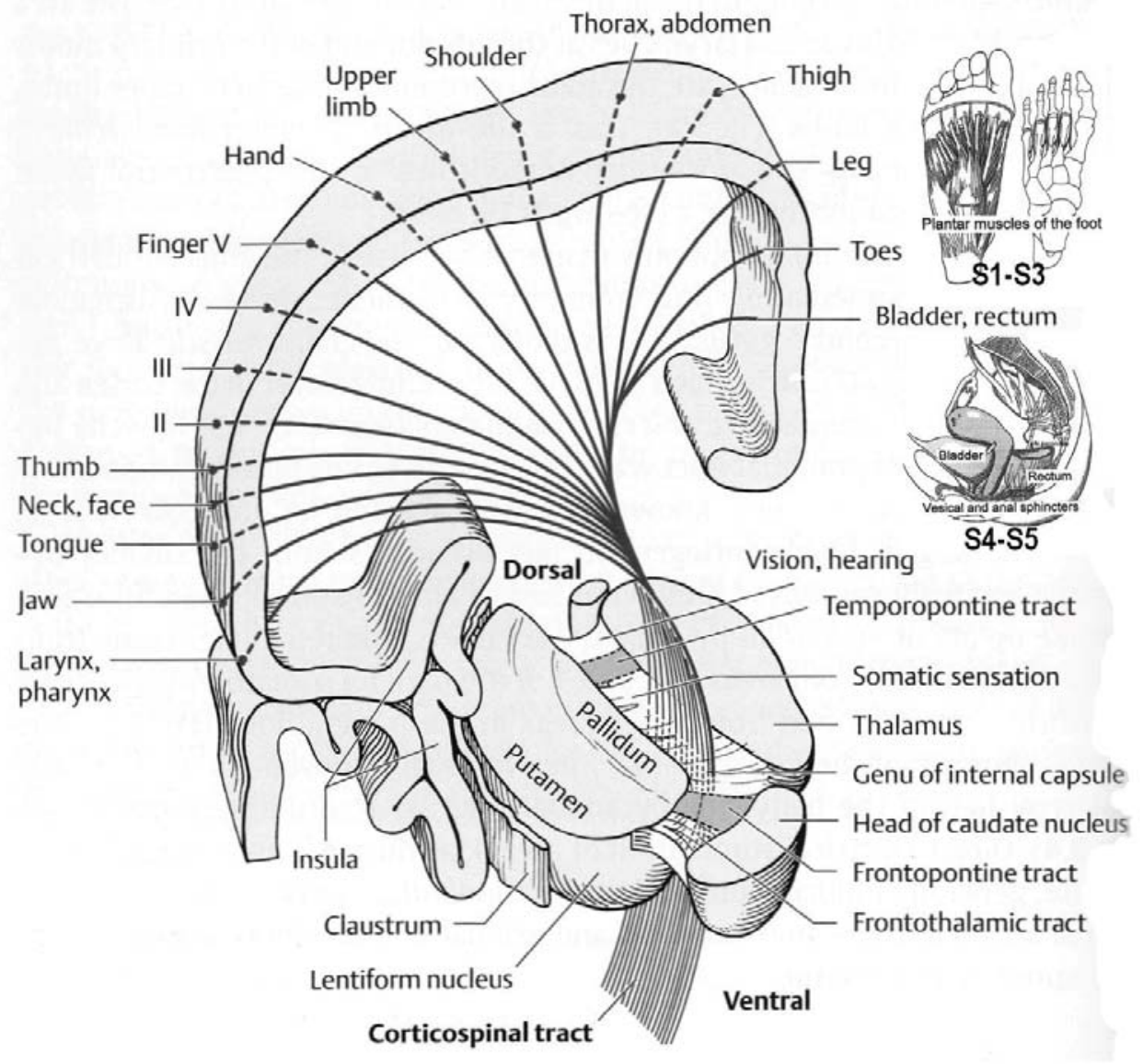

Figure 40: Primary motor areas (homunculus of the brain). Note that the toes are close to urinary bladder and rectum areas.

3.2.6. Power of reparative regeneration: The capacity for reparative regeneration is smaller, the higher an animal is on the phylogenetic scale [40,41]. Rats and dogs can have their nerves severed and not sutured together, yet their regenerative efforts are so strong that nerve continuity is restored and motor and sensory return will occur [40,42]. For a gap of $8 \mathrm{~mm}$ after transection more than $50 \%$ of the nerve fibers could be counted in the distal stump of the rat sciatic nerve [44]. In the dog, nerve fibers can cross a gap of 4 to $5 \mathrm{~cm}[43]$.

In human the power of regeneration is much smaller and the distances of regeneration are much larger than in most laboratory animals. The power of regeneration is small in the CNS and in the PNS it is different in different body parts. Well known for dentists is, that the nervous alveolaris always regenerates if the nerve is damaged with an injection needle. The Author experienced himself that in the head touch afferents were able to cross a gap of a few millimeters. Pain afferents seem to be more powerful in regeneration than touch afferents. But from nerve sutures of arm and leg it is known, that the nerves have to be adapted for regeneration. The radial, median and ulnar nerves have a different capacity for regeneration.

To draw conclusions from the regeneration capacity of the rat brain or tissue culture to the human PNS or CNS is therefore only justified, if differences in the regeneration capacity are taken into consideration. On the other hand, in animals one has all possibilities for research. However, comparable studies are necessary to know to what extent animal data can be used in human cases.

Short regeneration distances seem to be important in the human CNS, since there are no leading structures in the CNS as in the PNS. The oligodendrocytes do not guide in the same way as Schwann cells. 
In spite of all the problems in repairing the human CNS, still the human CNS can be repaired by movementbased learning. May be the capacity of repair is increasing dramatically if new cells could be built from stem cells with aggressive and long-lasting therapy.

3.2.7. Increase of motor units for repair: There are also differences in the increase of motor units after partial denervation of muscles between rats and monkeys. The rat can increase the motor unit by collateral sprouting of nerve fibers in the muscle by about $400 \%$, but the monkey only by about $50 \%$ to $100 \%$ [45]. This means the monkey (and most likely also the human), needs nearly the whole number of motor fibers to keep important functions [46] and muscle power.

3.2.8. The problem of muscle power generation: In incomplete cervical SCI ( $50 \%$ injury) sufficient muscle power can be developed by the patient to walk, run and jump. In nearly complete cervical SCI (95\% injury), the generation of sufficient muscle power is a fundamental problem. Due to limited regeneration, the patient can move different leg muscle and can even walk/move on treadmill (Figure 23H). But the patient is still far away from walking without weight-bearing support. The problem of muscle power generation can partly be quantified by the maximum load against which a patient can exercise on the special CDT device. In $95 \%$ cervical SCI, upon 0.5 years of CDT the patient can turn on the special device against a maximum load of 30 Newton and upon 5 years of CDT against $50 \mathrm{~N}$. The patient with the $50 \%$ cervical SCI could exercise already against a load of $200 \mathrm{~N}$ after 2 years. For $50 \%$ cervical SCI, Figure 21 shows the different reductions (improvement) of the coordination dynamics (CD) values for exercising against loads between 20 and $150 \mathrm{~N}$. At a load of $20 \mathrm{~N}$ good values were achieved after 0.5 years and for a load of $150 \mathrm{~N}$ more than 3 years were needed.

The patient Nefeli with a SCI of approximately 70\% became able to exercise against a load of $50 \mathrm{~N}$ after 8 months of CDT. But good values for 20 and 50N CD were not achieved within 8 months.

3.2.9. Plasticity: Sperry transposed the nerve supply of flexor and extensor muscles in the rat [61] and in the monkey [62]: the monkey relearned the task, the rat did not. Monkeys also differ from dogs [47] and rats [62] in the physiotherapy they need. In Sperry's experiment on monkeys, their learning to flex or extend the elbow in one situation did not necessarily become generalized to other performances. This indicates that the neural readjustment was not localized solely in the spinal centers, but involved reorganization at the supraspinal levels [48]. Surprisingly few trials were required for poliomyelitis patients to use transposed tendons successfully. The visualization of the task seemed to be the prime aid to the patients [63]. Intensive neurotherapy gives more useful functions of the to be re-innervated body parts $[63,49,50]$.

3.2.10. Loss of spinal cord plasticity due to pressure and ischemia: A loss of interneurons and spinal cord plasticity can occur directly with the spinal cord trauma, when the blood supply of the spinal cord [64] is interrupted [51-53], or with edema or cord pressure which causes ischemia mainly within the first 6 hours following the acute trauma (Figure 5). In the patient Nefeli mainly the bleeding following the surgery (and some tissue) caused pressure which gave rise to an incomplete SCI (Th10/11, Figure 36) after 3 days. Up to the level of Th9 also nervous tissue was lost dorsally (Figure 44C). Probably additional loss of interneurons and plasticity occurred.

In animal experiments it has been shown, that the motor cells located at the circumference of the anterior horn are more resistant to ischemia than those at the venter [54]. The motoneurons are nearer the arterial end of the capillaries, and the interneurons are nearer to the venous end. In slowed circulation, the peripheral cells use up the oxygen first, and the central cells are subjected to low oxygen levels. It may therefore happen that monosynaptic reflexes are still present, whereas the polysynaptic responses are lost [55], since they need the interneurons. Interneuron connectivity can partly be measured by polysynaptic reflexes in animals [56]. The secondary destruction of the spinal cord, following the first 6 to 8 hours after acute injury by edema or other pathologic processes, can possibly be reduced by localized cord cooling [56], or by hyperbaric oxygen [57], methylprednisolone, naloxone or dimethyl sulfoxide treatment $[57,58]$. 
Interneuron connectivity [59] may also decrease in the following months or years after the spinal cord trauma because of the denervation of interneurons by the injury of the spinal cord tracts. Interneurons may need trophic substance and activity [60] from afferents, other interneurons (mainly) or spinal cord tracts. Maybe the missing trophic effect of other neurons can be compensated transiently by an increased activity input. Partly damaged cord parts with loss of interneurons (Figure 41, indicated with dots), mainly surrounding the injury site, have to be activated by movement-based learning for not losing interneurons and spinal cord plasticity.

Even though the human CNS repairs itself by movement-based learning, the therapist has to understand the repair processes to optimize the repair, or, to enhance the efficiency of repair.

3.2.11. Repair of the injury site: The repair of the SCI has to bring the caudal spinal cord under supraspinal control again and the injury site has to be repaired. The repair of the injury site is especially of importance if the intumescentia cervicalis (arm and hand movement) and the intumescentia lumbosacralis (leg movements) (Figure 3C) are damaged, which was not the case in Nefeli. Her damage was mainly located in the thoracic spinal cord with the consequence of impaired trunk and pelvis control. Several movements for trunk and pelvis control had to be trained. These movements are those ones which patients have to train for disk prolapse repair and back pain reduction. Especially the up and down movement of the trunk is important (Figure 42) besides rotational movements.

3.2.12. Motor tract damage and its repair: Figure 41 displays a cross-section of the spinal cord. Some tracts and the approximate cord damage of the patient Nefeli are shown. The motor tracts in the spinal cord are anatomically and functionally segregated into two groups: a lateral group, comprising the corticospinal and rubrospinal tracts, and a medial group, comprising the reticulospinal, vestibulospinal, tectospinal tracts. The lateral tracts mainly project to the distal musculature (especially in the arms) and also make propriospinal connection. They are primarily responsible for voluntary movements of the forearms and hands, i.e., for precise, highly differentiated, fine motor control. Since the SCI is at the level Th10 (Figure 1C), the patient Nefeli can perform like a normal person precise, highly differentiated voluntary arm and hand movements. The medial tracts, in contrast, innervate motor neurons lying more medially in the anterior horn

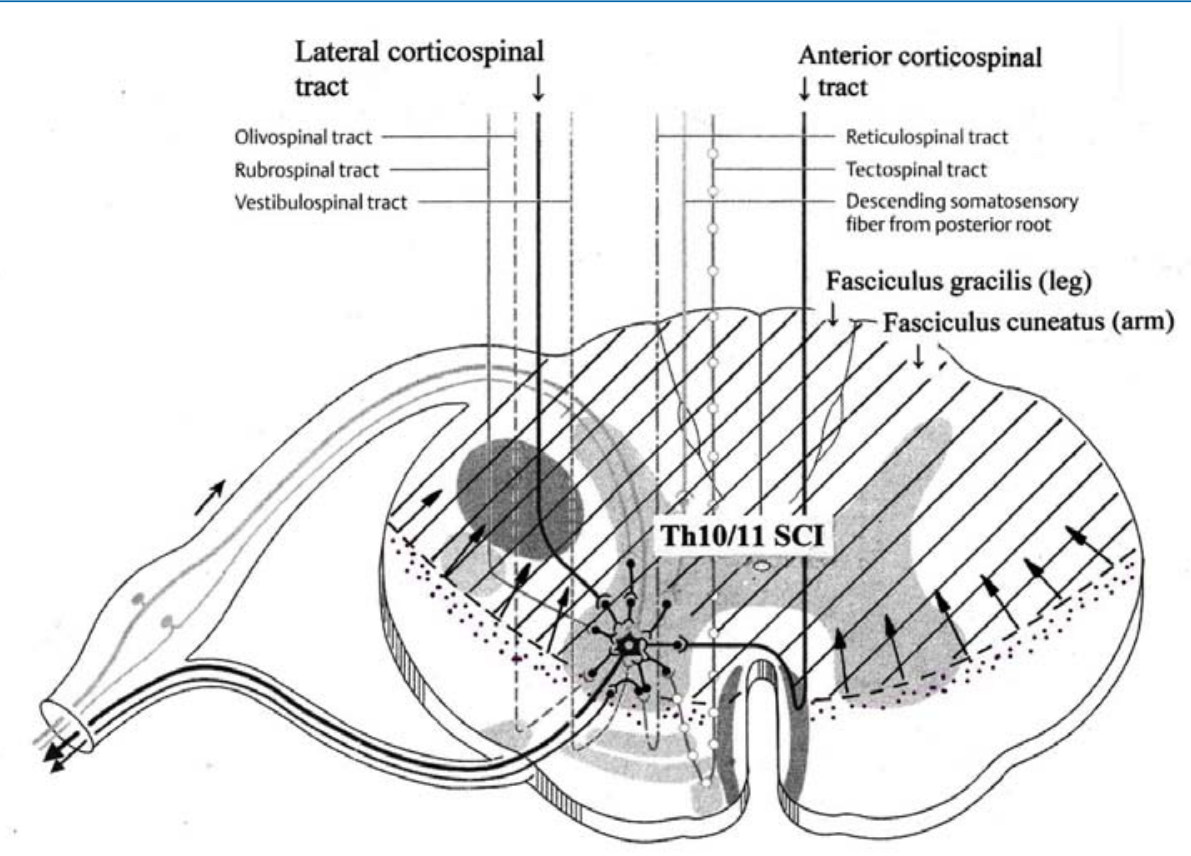

Figure 41: Spinal cord cross section with indicated approximate injury (cross-hatched) and loss of interneuron (dotted area) of the patient Nefeli. Synapses of descending motor tracts onto anterior horn neurons are shown. 


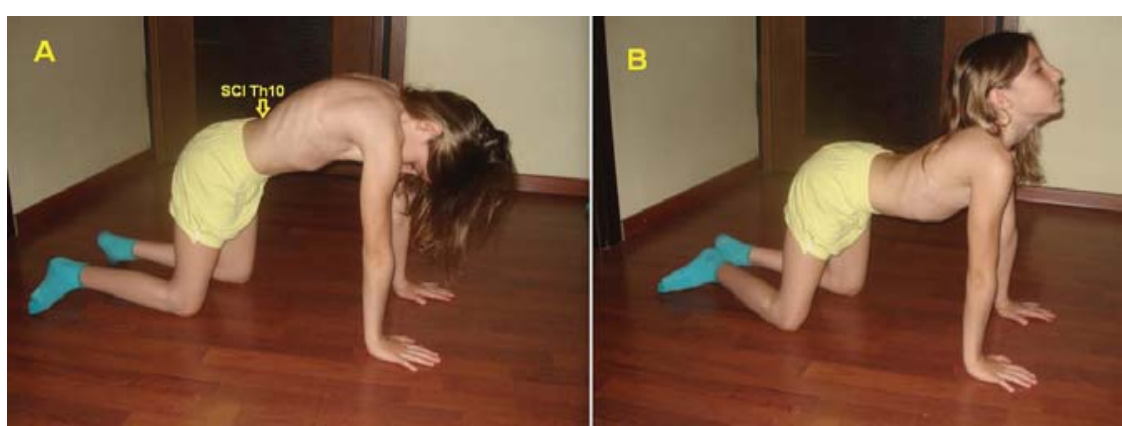

Figure 42: Up and down movements of the trunk to arch the patient's back and to activate neurogenesis at the SCI level Th10. The impaired arch is marked with SCI Th10.

and make relatively long propriospinal connections. They are primarily responsible for movements of the trunk and legs (stance and gait). These medial tracts are more ventral in the anterior horn (Figure 41) and are probably only little damaged. They will activate the motoneurons in the intumescentia lumbosacralis (not damaged) for stance and gait. Therefore, Nefeli has spared tracts to activate on volition leg and foot muscles. But the contributions from the medial group, lying in damaged cord area (Figure 41), are strongly reduced below the level Th10.

Many motoneurons in the intumescentia lumbosacralis to activate foot and leg muscles (Figure 41) are not reaching the threshold to generate an action potential because of too little input. Improving the coordination of the afferent input (Figure 43) by exercising on the special CDT device will help to reach the

\section{Neuron as a coincidence detector}
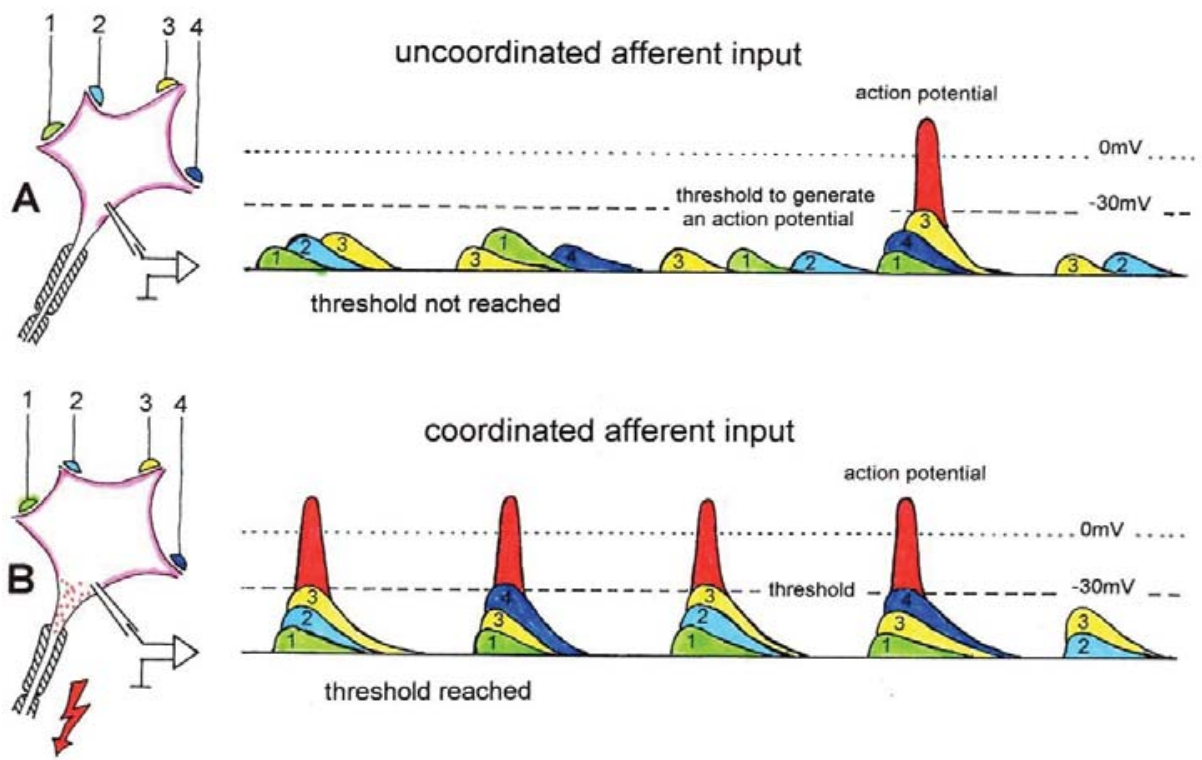

Figure 43: Neuron operating as a coincidence or coordination detector. A. Afferent input is reaching rather uncoordinated the cell soma. Only sometimes an action potential is generated, because the threshold of action potential generation is mostly not achieved. B. The action potentials in fibers 1 through 4 are reaching time-coordinated the dendrites or the cell soma. The postsynaptic potentials add up and the threshold is achieved at approximately $-30 \mathrm{mV}$, and action potentials are generated time-coordinated at the axon hillock. In the real CNS mostly, many more smaller postsynaptic potentials will contribute to the generation of an action potential and passive conduction from the dendrites to the cell soma has to be taken into account. Coordinated afferent input may thus induce or enhance (coordinated) communication between neuronal network parts following CNS injury. 
threshold. The improvement of the coordination of the afferent input will only partly help. Enhancing the strength of the several thousand synapses onto the motoneuron (Figure 41), by exercising, will additional help. Still more input from the medial and lateral motor groups are needed. As found above, the spinal cord of Nefeli partly regenerated. More tract fiber input made the motoneurons to reach the threshold and activate leg and foot muscles.

As analyzed above, it unlikely that a substantial number of fibers, of the for example pyramidal tract (lateral corticospinal tract), regenerated over the injury site to grow down to the motoneurons in the intumescentia lumbosacralis. Probably relay neurons or neural networks were partly built at the injury site. Axons from these new neurons grew down to the motoneurons. The problem with this assumption is, where are the stem/progenitor cells are coming/migrating from.

Another possibility is that new tract neurons were built anew in the somatosensory cortex. Their axons may grow along the anterior corticospinal tract fibers, passing though the not damaged cord part at the injury site and reaching thus the motoneurons in the intumescentia lumbosacralis (stance and gait) and the conus medullaris (urinary bladder and sexual functions). The regeneration times are not against the building of new tract neurons. But these repair possibilities would need more than a year to contribute to the repair. But there are many patients with a SCI for decades and regeneration or improvement of function did not occur. First, many spinal cord injuries are quite complete and there is not much space for the growing of nerve fibers through the injury site. Second, for the wanted afferent and efferent integration intensive efficient therapy is needed so that the axons of the newly built neurons grow to the wanted place. But third, in the developing (pediatric) CNS more nerve cells are built anew especially with aggressive efficient movement-based learning therapy.

3.2.13. Sensory tract damage and its repair: The posterior columns consist of the fasciculus cuneatus (arm) and fasciculus gracilis (leg) and transmit impulses arising in the proprioceptors and cutaneous receptors from arms and legs. As we can see from Figure 41, these columns are damaged because of the pressure injury of the spinal cord from dorsal at the level of Th10 (Figure 36). Since the pressure was exerted from dorsal and the spinal cord is fixed by the dentate ligaments (Figure 4), the damage is primarily dorsal. Therefore, the patient Nefeli should have problems in feeling the position of her legs (proprioception) and should no longer securely feel the floor under the feet (cutaneous receptors; touch and pressure). As a result, both stance and gait should be impaired (gait ataxia), particular in the dark or with closed eyes.

Indeed, Nefeli wants to train bare foot or wants to wear only socks to enhance the afferent input necessary for improving standing, walking and balance. She clearly said, when I started to feel more the soles of my feet my balance improved during walking and standing. Obviously, the sensitivity also improved with the regeneration of the cord and reorganization of the brain. With ongoing therapy, she managed also to walk with shoes at school (Figure 32); even though the sport teacher sometimes let the pupils walk and run with socks to include Nefeli better in the sports program. The two-point-discrimination of the sole of the foot was $15-25 \mathrm{~mm}$ and in the healthy sister approximately $10 \mathrm{~mm}$.

Figure 44 shows the fasciculus cuneatus and the fasciculus gracilis. The SCI Th10 is indicated and is sited caudally to the start of the fasciculus cuneatus. As Figure 44C indicates, a regeneration is rostral more difficult because the gap at the injury site is broader there. Also, leading structures for regeneration are missing. Regeneration and or building of neuronal networks at the injury site will start from ventral; where the distance for regeneration is shorter, where healthy cord tissue remained as a leading structure for nerve fiber growing and may be where neurogenesis could be induced by membrane depolarizations from the remained by movements activated cord tissue.

Regenerating sensory fibers from the legs would have to cross the injury gap and grow along myelin sheaths, generated by the oligodendrocytes, to the nucleus gracilis. As for the regeneration of motor fibers across the gap, mismatch will occur. The nucleus gracilis contains the second neurons, which project their axons by way of the medial lemniscuses to the contralateral thalamus (Figure 44B). The somatotopy will be disturbed and has to be compensated for by the plasticity of the brain. The sensory information processing from the arms through the nucleus cuneatus is not impaired (Figure 44). 

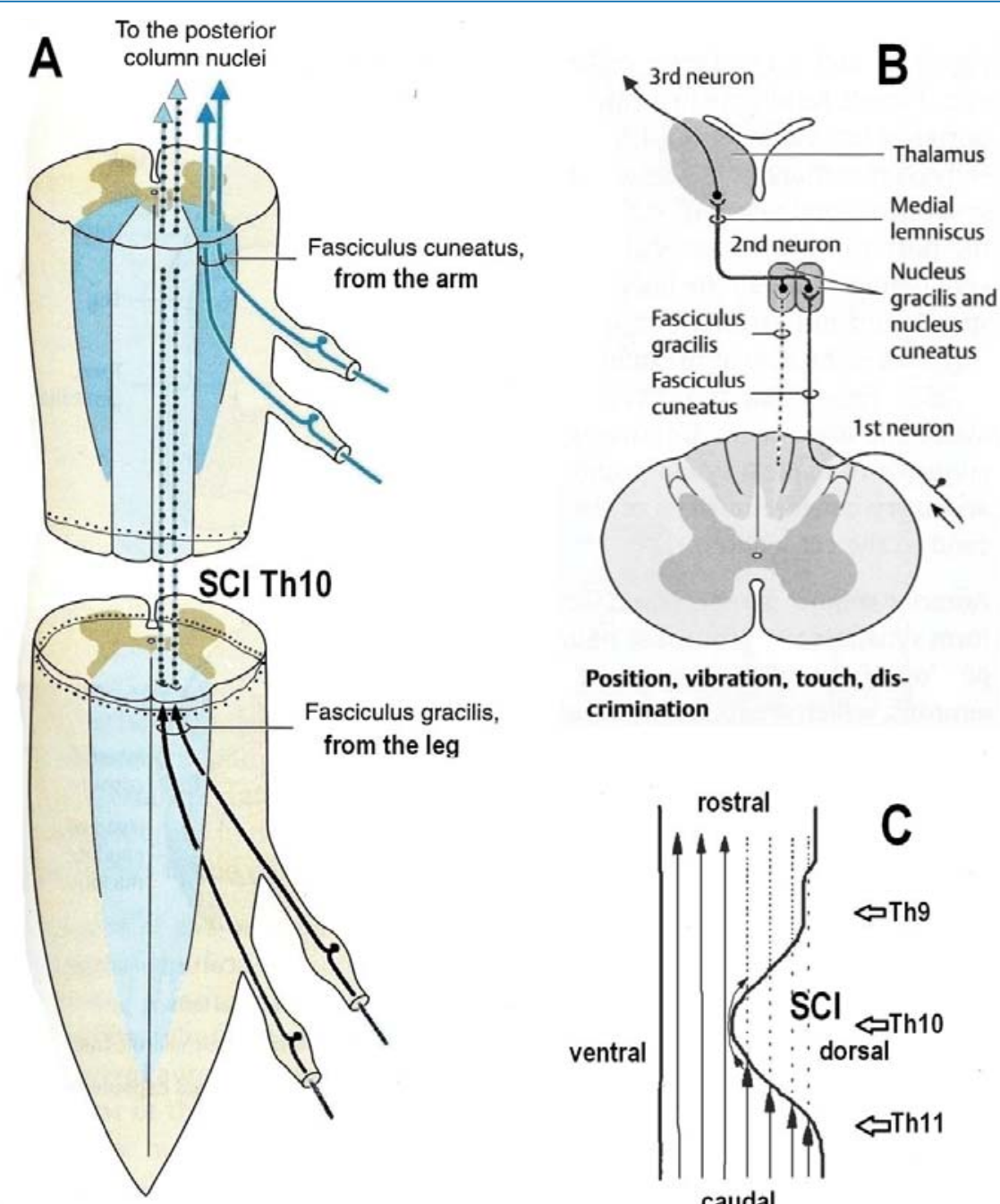

Position, vibration, touch, discrimination

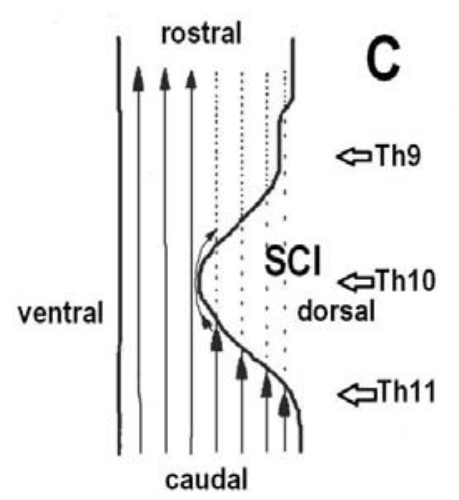

Figure 44: A. Posterior funiculus, containing the posterior columns fasciculus gracilis (medial, afferent fibers from the leg) and fasciculus cuneatus (lateral, afferent fibers from the arm). Afferent fibers sub-serving different sensory modalities traverse the root entry zone and enter the posterior horn (B). The type of myelin changes from peripheral to central, and the myelinating cells are no longer Schwann cells, but rather oligodendrocytes. The SCI at the level of Th10 is indicated. The afferent fibers from the arm join the cord at cervical levels and lie more laterally and dorsally to the SCI. The action potentials derived from receptors in muscles, tendons, fasciae, joint capsules and skin are conveyed in the distal processes of pseudo-unipolar neurons in the spinal ganglia. The central processes of the cells, in turn, ascend in the spinal cord and terminate in the nucleus gracilis of the medulla oblongata. The impulses derived from receptors in the leg are conveyed similarly to the caudal spinal cord. But in the spinal cord the central processes are mainly destroyed at the injury site and the action potentials cannot reach the second neurons in the nucleus gracilis of the medulla oblongata (dotted lines) (B). B. Central continuation of posterior column pathways. The posterior column nuclei contain the second neurons of the afferent pathway, which project their axons to the thalamus. Due to the SCI the second neurons in the nucleus cuneatus are partly de-afferented (dotted line). C. Pictured SCI site. Soled lines are functional, dotted lines not. To understand the regeneration of the human spinal cord, induced through CDT, more knowledge of the regeneration in human is needed.

\subsection{Case report of spinal cord repair in the patient Nefeli (70\% SCI) via coordination dynamics therapy (CDT) up to 1 year}

3.3.1. Cancer and spinal cord injury treatment till CDT was started:_After documenting details of the regeneration of the spinal cord in the patient Nefeli, the improvement of CNS functions via movement-based learning will be given.

When Nefeli was 5.5 years, it seemed that she suffered pneumonia because of coughing. She got antibiotics and the coughing stopped within two days. Because this was a too quick recovery a thorax 
picture was made. A carcinoma was found paravertebral. It was a neuroblastoma growing from the ganglion Th10 on the right side. The cancer was therefore quite close to the spinal cord and the vascularization is high in this area, especially when a tumor was growing there. The tumor, $8 \mathrm{~cm}$ in diameter, was removed in Greece. The father asked for an additional neurosurgeon in addition to the orthopedic surgeon, because the tumor was connected to the spinal canal. He wanted to pay for the additional costs. Both surgeons refused for a combined operation, even though when removing an acusticus neurinom sometimes a neurosurgeon and an HNO physician do cooperate. After the operation Nefeli complained because of pain in the legs and was crying all the time. The other five children in that hospital room could not stay this continuous crying of Nefeli and moved out of the room. The physicians argued that it is normal after an operation. Clinical knowledge is that if a patient continuously complains of pain one has to check the cause, even if the physician does not believe that the patient has really pain. After three days of pain, the pain disappeared; but the urinary bladder stopped working and Nefeli could not stand any more. The parents took her to another hospital to get an MRI done. The MRI showed a spinal cord compression at Th10/11 levels from bleeding and some tissue. The spinal cord was freed to enable complete decompression, 3.5 days after the first operation. The decompression was re-assessed the following day with a new MRI. But Nefeli had suffered already an incomplete SCI at Th10/11 levels (Figure 36). From disk prolapses it is known that if soft pressure onto the spinal cord lasts longer than 24 hours, the spinal cord becomes damaged. The medical malpractice was mainly not to check for bleeding into the spinal canal at a time when the child was crying and complaining because of pain.

With four months neuro-rehabilitation in Switzerland, which cost approximately 150000US Dollar, Nefeli improved a bit, but mainly due to spontaneous recovery according to the knowledge of the Author. A year later Nefeli was send again from Greece to Switzerland for another four months neuro-rehabilitation. The costs were again approximately 150000US Dollar. The special CDT device was not used in that children's neuro-rehabilitation place (Affoltern), even though they knew about it. Urinary bladder function did not improve and the progress in movements was limited. Nefeli refused to have conventional physiotherapy at home in Greece. At school she had to use sticks and orthosis (Figure 32B). Because of no physiologic bladder functions, she was using diapers. An assistance helped her to manage at school.

At an age of 9 years, three years after the first operation with "medical malpractice", the father was advised by a professor in Greece to try CDT and started it. Spasmolytic drug and urinary bladder medication were stopped. Following four weeks of aggressive CDT, supported walking and jumping improved. The crawling became better.

Urinary bladder functions started to improve. The sacral micturition center started to work. When the urinary bladder automatism (S2-S5) was activated by the CNS, the residual urine became less than $10 \mathrm{ml}$ as measured by catheterization. Mostly the patient felt the wish to void. But the time from the first desire to void till to the activation of the bladder emptying automatism was one to three seconds. All bladder functions were working unregularly. With regeneration mostly functions re-appear unregularly. The patient still had bladder infections.

When the Author controlled the data of the cancer removal, he found out that the cancer treatment had been far from optimal. Probably the neuroblastoma of the ganglion was not fully removed, even though this would have been possible. In the Authors cancer extirpation, the whole cancer was removed and $1 \mathrm{~cm}$ healthy tissue around the glioblastoma to be sure that the whole cancer tissue was taken out. In Nefeli four lymph nodes were removed in the cancer area; three had a metastasis. In the Author, the two lymph nodes with metastasis were removed and further four ones leading away from the cancer (in this case the neck) with no metastasis. Radiation and chemo therapy was administered to the Author but not to the 6-yearold child, mainly because of the SCI. Obviously, the cancer operation in Switzerland (Author) was much more thoroughly performed than those in Greece (Nefeli). As the urine test of decreasing catecholamine metabolites indicated, there seem to be no active growing of the neuroblastoma and/or metastases in Nefeli. A newly made MRI indicated that there might be tumor tissue remained, but no active grows could be found 
in the area of the former Th10 ganglion. The intensive CDT may have activated tumor suppressor genes. But the movement-based learning therapy is most likely not sufficient efficient to stop aggressive cancer growing. Pathologists had the opinion that the cancer was not very aggressive. In the Author two check-ups of the whole body for metastasis were performed (PET). Nothing was done so far in Nefeli. As stimulated by the Author, more diagnostic of possible tumor growing should be done to be on the safe side. But to think that the Author obtained optimal cancer treatment, is also wrong. When the region of the former cancer below the upper lip was radiated, the eyes and the brain were not protected against radiation. The Author asked for protection, but it was refused.

More causal cancer treatment is needed, including progress in genetics and epigenetics. Interdisciplinary research and treatment have to be improved. Existing conventional cancer treatment has to be administered more thoroughly.

3.3.2. Repair within 6 months of CDT: The repair of the SCI of Nefeli involved the repair of movements below the injury level, especially the walking, the repair of urinary bladder functions, the reduction of scoliosis and spasticity and last not least the repair of the sexual functions which will become relevant after puberty. Additionally, it has to be controlled whether the tumor is growing again.

As can be seen from Figure 36A the most loss of spinal cord tissue was at vertebra levels Th10/11. Figure 36B further indicates (spinal cord is white) that there was additional loss of spinal cord matter rostral to the Th10/11 levels. The loss of spinal cord matter was up to the vertebra Th8. Probably because of this additional loss of spinal cord matter, caused by the compression, Nefeli got a severe scoliosis (Figure 45), which is a medical problem and not just a cosmetic one, because of possible spinal nerve root compression in the intervertebral foramen in the future. Most SCI patients get a scoliosis, but not such a severe one.

Figure 46 shows once more the starting position of CDT. After conventional neuro-rehabilitation she needed sticks and orthosis and had to sit quite often. The urinary bladder was not functioning. A normal school life was not possible. Nefeli often said, I want to be again like the other children at school. Following 6 months of therapy, she could walk again (Figure 47) and could manage much better at school. She could walk to the whiteboard and write there (Figure 32C,D).

The walking performance was still far away from normal. As can be seen from Figure 47B, the right knee is inwardly rotated. The outward rotation of the right foot during walking cannot be seen in this picture. The scoliosis can even be seen through the clothes. When walking with sticks (Figure 47A), no scoliosis is apparent, because of the stretching of the upper trunk.

Nefeli had in the legs extensor spasticity, flexor spasticity and

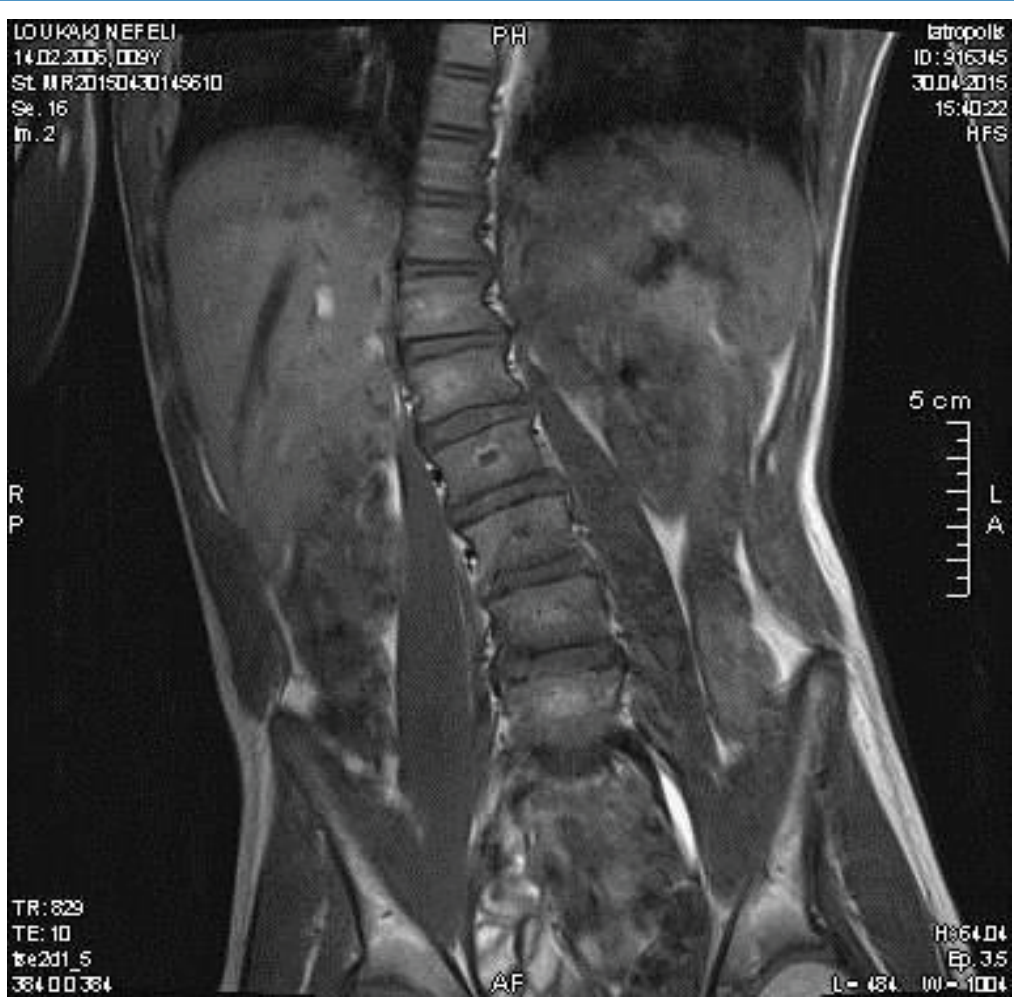

Figure 45: Severe scoliosis of the SCI patient Nefeli caused by the SCI and the cancer removal. With the removal of the neuroblastoma at least one intercostal was removed. 


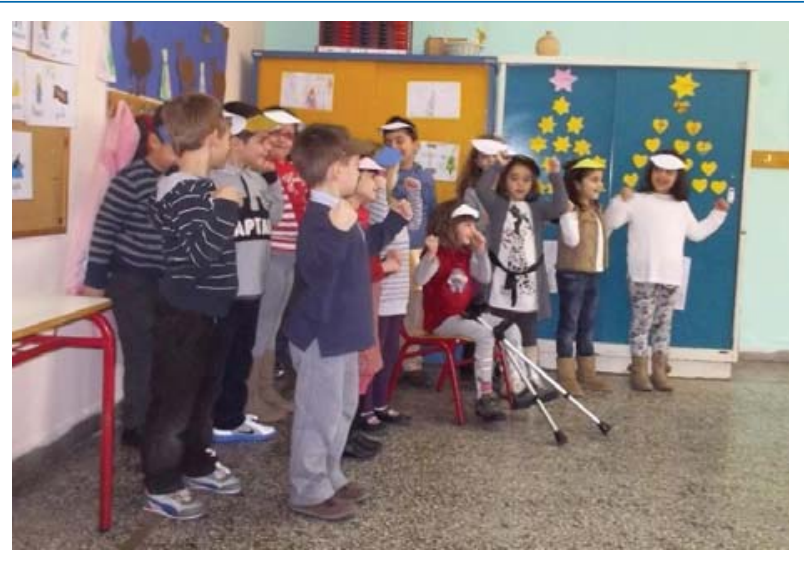

Figure 46: The six-year-old Nefeli (sitting), who acquired a SCI, following 8-months-therapy in a rehabilitation cevnter in Affoltern in Switzerland (one day costs approximately 2000 US Dollar). Note, Nefeli had problems to manage at school in the first class. of the urinary bladder (co-contraction of the detrusor muscle and the external bladder sphincter), which made it easier to improve bladder functioning. Exercising on the special CDT device and the jumping on springboard strongly activates and trains urinary bladder and bowel functions. Because of the rhythmic and dynamic load changes, exerted onto the pelvic floor during jumping, the external bladder and anal sphincters (somatic muscles), as a part of the pelvic floor, are rhythmically and dynamically activated and relaxed, and passively stretched for movement-based learning. The inner sphincter (smooth muscle) is probably trained and activated by the pressure changes in the detrusor muscle.

At school Nefeli was using diapers. When going to the WC for disabled, a helper was assisting. At home during CDT she was not using diapers. Often, she got wet and the trousers had to be changed. The time from the urge/desire to void and reaching safely the WC improved. Figure 48 shows the improvement of urinary bladder functioning by the times she reached the WC safely and could keep the fluid. The times to hold the fluid longer improved but varied quite much. Once she was even be able to hold the fluid for $20 \mathrm{~min}$. Also, the emptying of the colon and rectum (defecation) became more regular.

When training regularly, Nefeli had no dyssynergia of the bladder. That means, when the detrusor (smooth muscle) contracted and the inner sphincter (smooth muscles) opened, the external sphincter (striated (somatic) muscle) relaxed and the fluid could leave the bladder via the urethra (Figure 27). But when she was performing the program unregularly (not according to the program), it happened sometimes that she got very spastic in the legs and pelvis. Then also the external bladder sphincter got spastic and she could not void even though she had the desire to void. By exercising a bit on the special CDT device and jumping on springboard, the external bladder sphincter relaxed and she could empty the bladder physiologically again.

This shows that even though the bladder functioning improved, still a physiologic storage and emptying was not established in the long-term memory. The repairs of the vegetative nervous system and especially those activating bladder functions (in coordination with the somatic nervous system division) need time. SCI treatment for children should be able to repair the urinary bladder so that they have a private sphere in five or ten years.

The improvement of motor functions from wearing orthosis and walking with sticks (Figure 46) to free walking without orthosis (Figure 47) is quantified by the re-learning of free walking and supported jumping on springboard. 


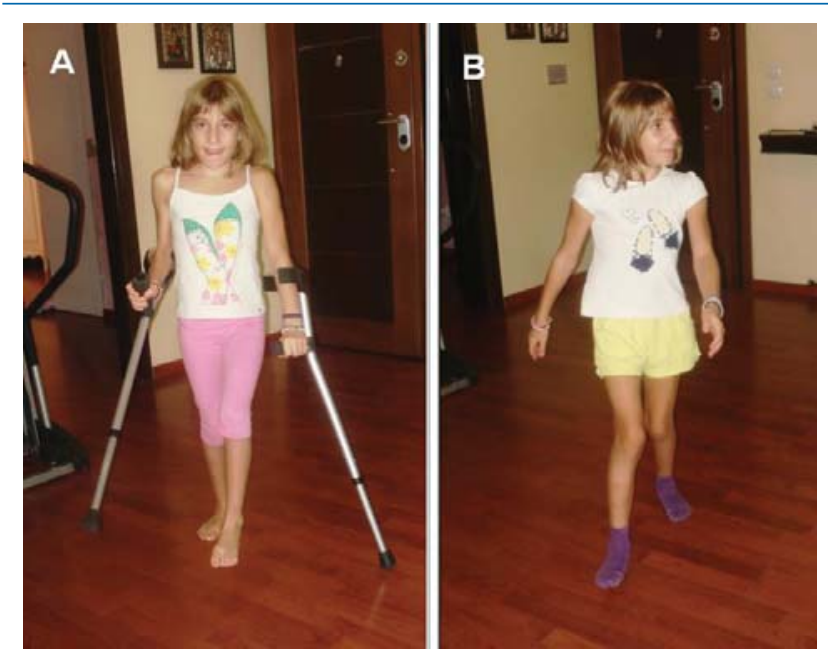

Figure 47: The girl Nefeli, who acquired a SCI at the levels Th10/11 during walking with sticks and free walking. Note the pathologic walking pattern in B.
Figure 49 shows the beginning and improvement of free walking on treadmill in the forward direction. After improving the walking on treadmill at a speed of $2.5 \mathrm{~km} / \mathrm{h}$, it was changed to a higher speed $(4 \mathrm{~km} / \mathrm{h})$, which is more difficult but more close to a real walking speed. The walking period was limited by losing the balance. The problem was therefore not to stay in the walking pattern, but to keep the balance for a longer time. Another 13-year-old cerebral palsy patient (Rafaelena), training partly together with Nefeli, could not learn to keep the balance during free walking on treadmill. One has to remember that treadmill walking is different to normal walking (more difficult for patients), because the kinetic energy in the forward direction is missing with the consequence that it is more difficult to keep balance. This can be observed in the mountains. If an ibex or mountain goat has to cross a difficult and steep piece of ground, it is running. If one or two steps cannot be performed properly, they will not fall down the steepness, because the kinetic energy is bringing them further to a not so difficult piece of ground.

To improve the forward walking, the backward walking was also trained for symmetry improvement. Forward and backward walking were trained together. Figure 50 shows the improvement of forward and backward walking quantified by the number of performed steps till the patient lost the balance. It was much more difficult to walk backwards and the speed had to be much lower $(1.7 \mathrm{~km} / \mathrm{h})$ than for forward walking $(4 \mathrm{~km} / \mathrm{h})$. Still it seemed that the backward walking improved the forward walking. Since it was for Nefeli much more difficult to walk on treadmill than walk freely, she later on refused to train the free walking on the treadmill. But she further trained the normal free walking in the forward (Figure 47) and backward direction. The beginning of walking was hard work for the patient and the therapist (Author). Often Nefeli was falling during normal free walking. She never complained and was mostly laughing. As a para with an injury level of Th10, she had no problems with good protection reactions. And, of course, she had no overweight, which would make the walking of a patient with a SCI more difficult. With ongoing therapy, the balance of Nefeli during walking improved, but she still fell quite often (and the Author could not always catch her) because she liked to move fast and risked more. Her far goal in mind was the re-learning of running.

As can be seen from the Figures 47 till 50 , Nefeli preferred to walk during training without shoes, because she could walk better then. The enhanced input from the soles of the feet improved her walking performance. At school she had to walk with shoes. But, as can be seen from Figure 32D, she managed

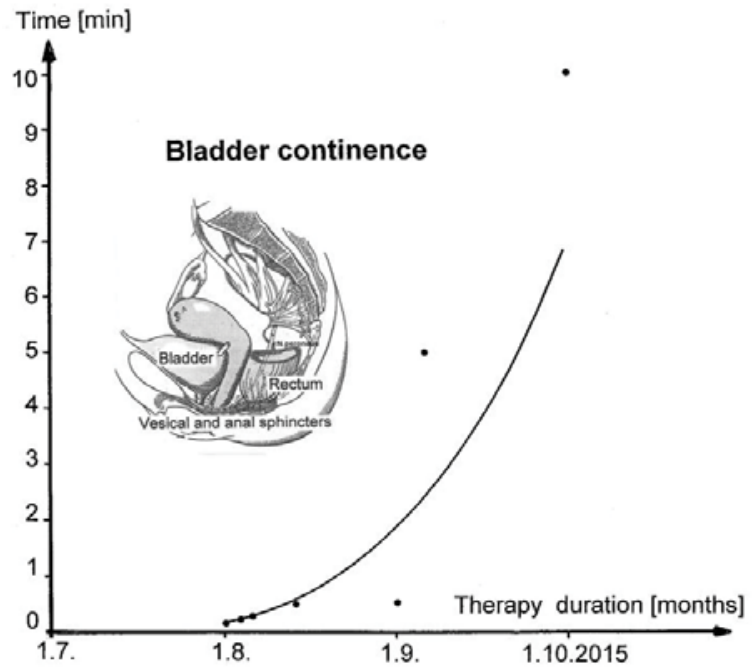

Figure 48: Improvement of urinary bladder functioning quantified by the time from the urge/desire to void until micturition. 

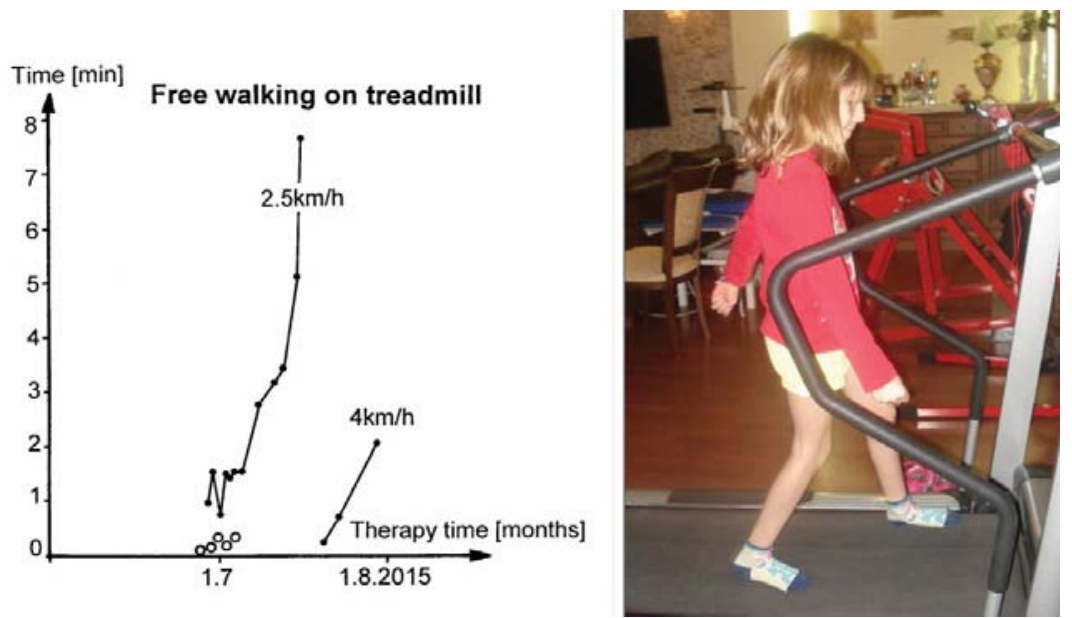

Figure 49: Improvement of free walking on treadmill of the 10-year-old patient Nefeli with a SCI. At the beginning a walking speed of $2.5 \mathrm{~km} / \mathrm{h}$ was only possible, later on a speed of $4 \mathrm{~km} / \mathrm{h}$ became possible. The patient walked without shoes to have a better sensory input and in this way a higher stability, which was the limiting factor of continuous walking.

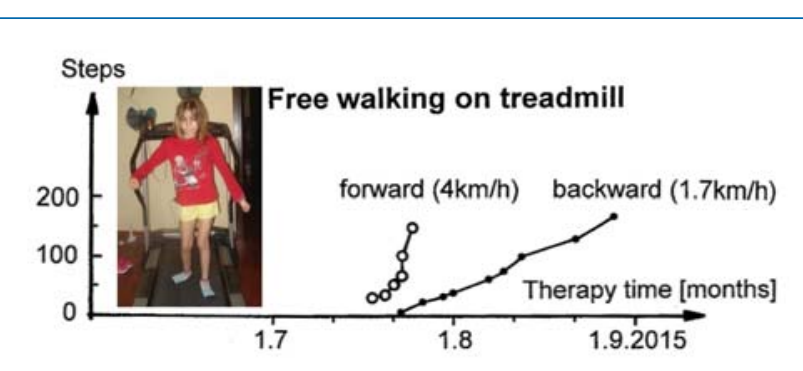

Figure 50: Free walking on treadmill in the forward and backward direction of a patient with a SCI. Note the pathologic inward rotation of the left knee and the not straight upper body because of the scoliosis. quite well also to walk with shoes. But the jumping was easier when she jumped without shoes (Figure 51).

Figure 51 shows the improvement of supported jumping in anti-phase. Even though Nefeli could jump without support, the supported jumping was preferred, because the movementinduced afferent input was then more physiologic. More physiologic afferent input makes it probably easier to re-learn physiologic movement patterns. But moving sometimes without support is also good, to 'tell' the CNS what is has to learn.

To repair the SCI as much as possible, Nefeli had to be made to train at her limits (aggressive therapy). But she also wanted to play with her sister and other children and she wanted to have an easier life like the other children. It was therefore difficult to push her to limits. Especially when a parent was missing, the Author was often not sufficient patient and struggled with her. In spite of all the hard work together, she well understood that she had to train to get better and she liked to train with the Author. She wrote into the protocol book that she likes the Author (Figure 52). Later on, she clearly said why she liked the Author, because he was fighting with her to make her a better life. In spite of all the "fighting", Nefeli was clever and understood exactly what was going on with the treatment; but she was still a child. Mothers with cerebral palsy children are in a much worse situation. They are fighting very much, are tremendously patient with the child, but only little is coming back from the child.

When the Author asked Nefeli, how was it in the rehabilitation center in Affoltern in Switzerland, she answered: It was nice there, I liked very much there - but it was a bit like in a playing school.

In spite of all the hard work, Nefeli got much better and she appreciated that. The question remained, to what extent can a SCI be repaired in children.

3.3.3. Repair within 12 months: The CDT was continued. When the Author trained with the patient, there was progress. When the Author was not training with her, there was little or no progress. Stability of standing and walking improved. Urinary bladder functions improved further. Apart from bladder function 

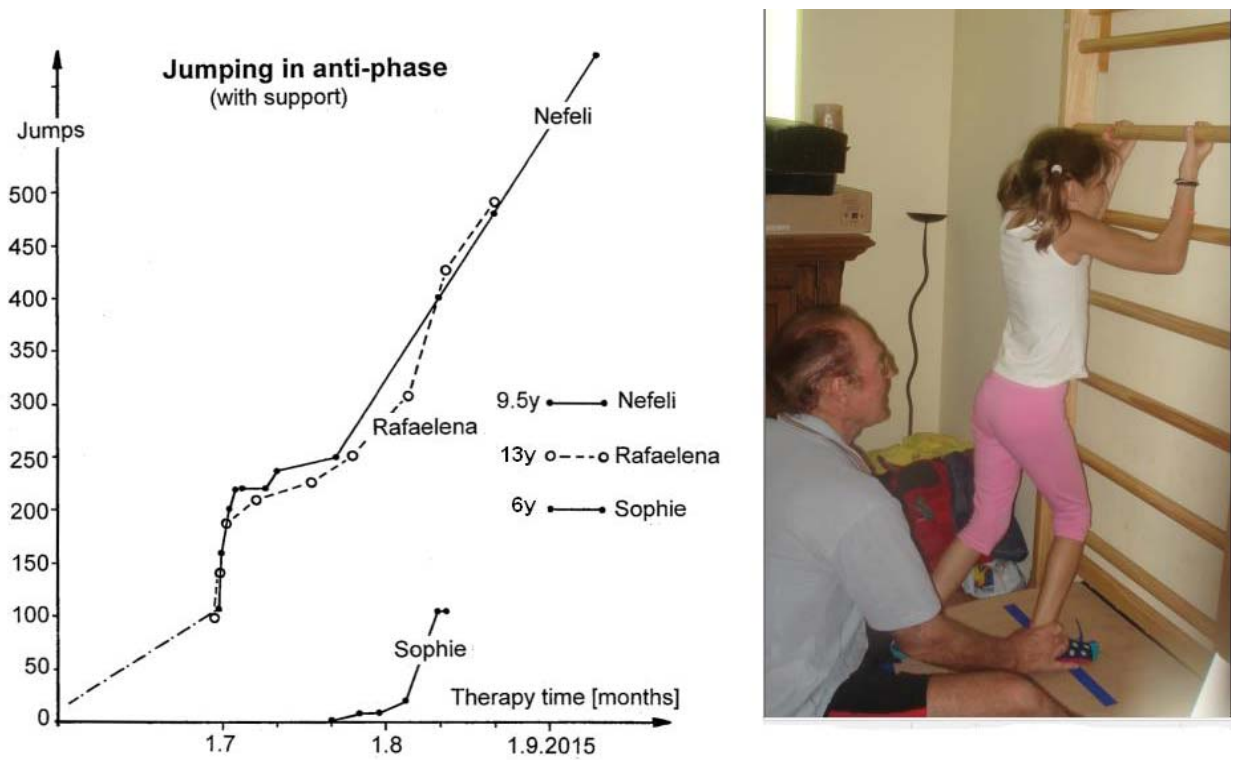

Figure 51: Supported jumping in anti-phase of the 9.5-year-old Nefeli with SCI in comparison to other girls. Note, with the right small finger the therapist (Author) is trying to keep the right foot of Nefeli in a physiologic position. The progress in jumping is quantified by the increase of jumps per series. $y=$ age in years.

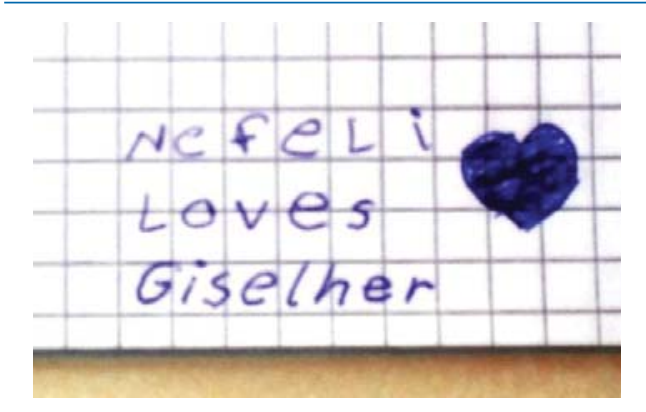

Figure 52: In spite of all the struggles the Author had with the patient Nefeli when pushing her to the limits of exercising, she wrote into the control book that she likes the Author.

repair, the main problem seemed to be the foot functions. The induction of the stepping automatism (Figure 53), located mainly in the intumescentia lumbosacralis, for improving leg functions by learning, was not possible following 9 months of CDT. She could not lift sufficiently the knees and dorsal flex the feet for heel strike.

After 10 months of CDT, the sensitivity of the soles of the feet improved (dermatomes L4 to S1, (Figure 1)), which is important for controlling walking and jumping. She became able to dorsal flex the feet (Figure 54) with little power. She became able to move a bit the toes, but all together. But standing on the forefoot was only possible with the help of the extensor spasticity.

The use of spasticity for certain functional aspects is not of interest for a full functional repair which was the far goal.

Nefeli learned to run a bit with poor performance. When falling she became able to get up from the floor by herself without support (Figure 55). When she was falling, the protection automatisms worked well so that she was not afraid of falling. For getting up from the floor two patterns were used. Nefeli could mainly use the arms for getting up (Figure 55A,B,C,D) or use the legs (Figure $55 \mathrm{E}, \mathrm{F}, \mathrm{G}, \mathrm{H})$. When using the legs, she had to push herself up. This getting up with momentum was fast and made the hands in Figure 55H blurred. For the repair of the CNS the getting up with the legs is more beneficial because

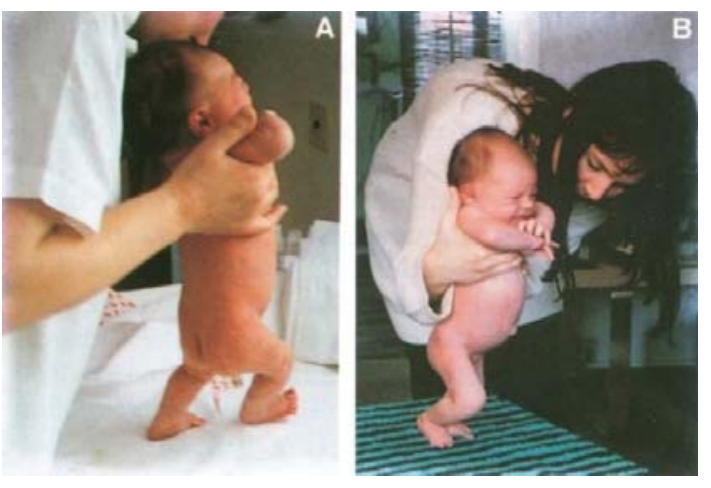

Figure 53: Automatic stepping in a newborn infant. A. The 5-day-old infant, Juliane, performing primary automatic stepping; slight backward posture. The heel of the right foot touched the ground first. B. Infant Juliane, 8-day-old, performing automatic stepping. 

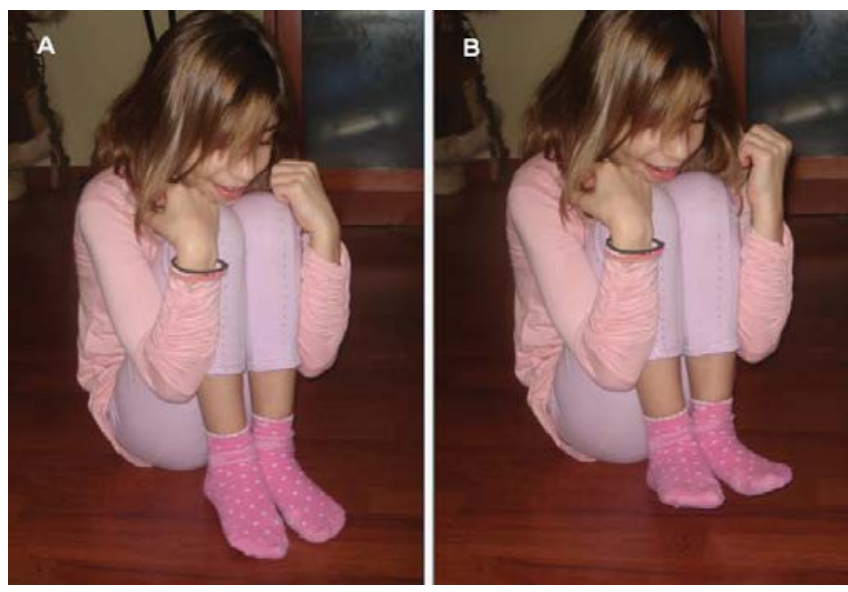

Figure 54: Volitional dorsal flexion of the feet in a patient with 70\% SCI. In 'A' plantar flexed and in 'B' dorsal flexed. To improve foot power, the hand grip is co-activated (clench her fists). the legs are trained more. Good balance was needed for both patterns.

Nefeli became able to regularly turn on the special CDT device against $50 \mathrm{~N}$ and became able to use the whole repertoire of movements on a special CDT device (Figure 56). The repairs of the coordinated firing of neurons deeper in the complexity of CNS neural network organization improved. Nefeli was trying out any kind of movements she was able to perform and showed them proudly to the parents (repair phase of regeneration). Since Nefeli from Greece was clever and had a good memory, she realized what functions recovered anew. She realized what hard work was necessary to get more and more functions back. The problem was that she was going to

school and had not sufficient time for training. She had problems to understand that the training is more important than playing and going to school. Losing one or two years of school would not change her life. Later on, during life, the rate of learning is reduced, but since she will be more matured then, she would benefit more from the learning (second way of education). On the other hand, a not functioning bladder is a 'horror'. One is concerned with bladder infections, catheterization and even with operations for the rest of the life. In Switzerland the school regulations are very strict; the school has always priority, what is not right.

3.3.4. Measuring the improvement of CNS organization non-invasively by means of coordination dynamics (CD) values : When Nefeli became able to turn continuously on a special CDT device at $20 \mathrm{~N}$ and was accepting it also, the measuring of the improvement of CNS functioning became possible. Figure 57
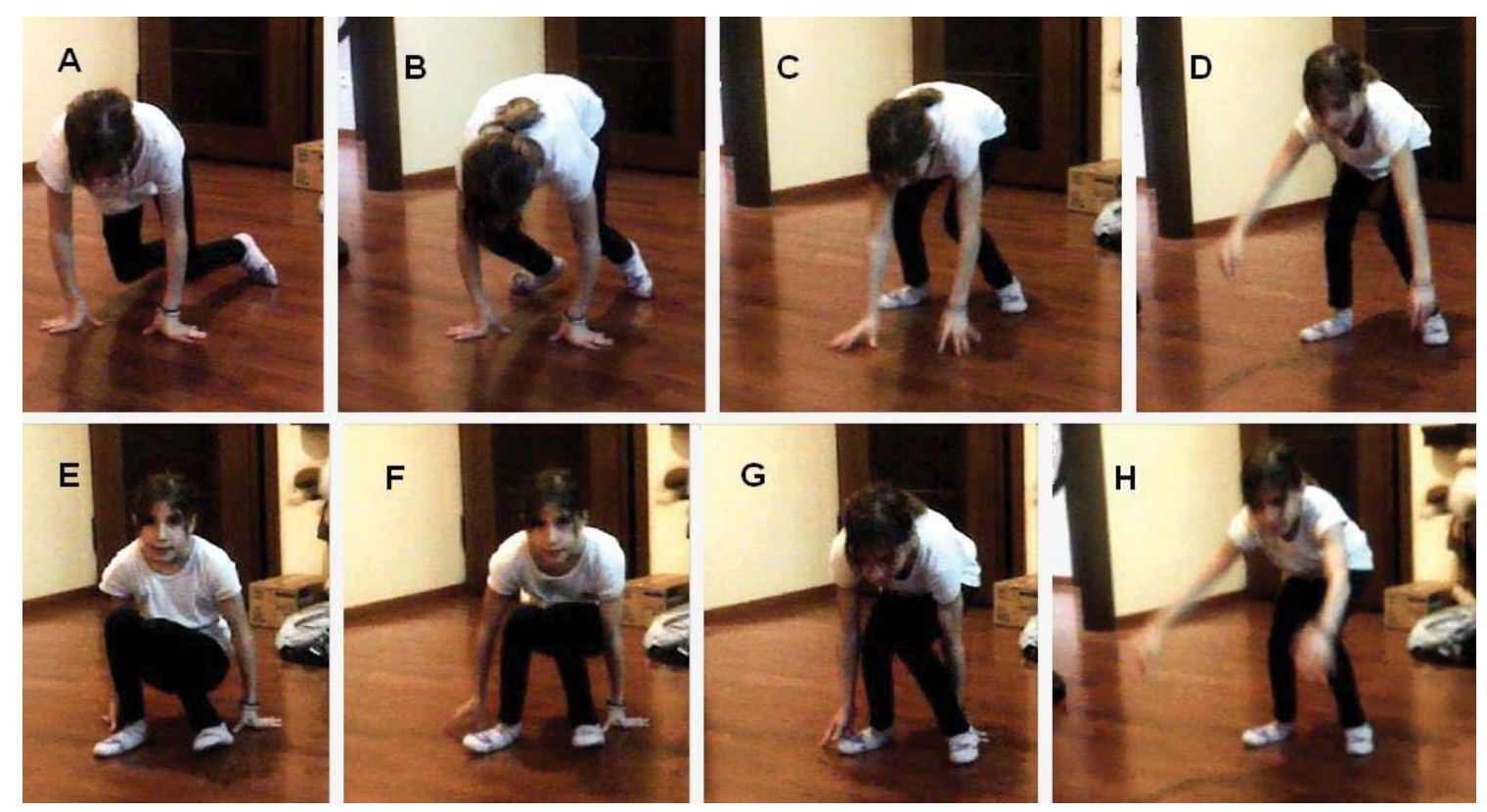

Figure 55: Two ways to get up from the floor after falling. In A through D mainly the arms are used for getting up and in E through $\mathrm{H}$ the legs. 


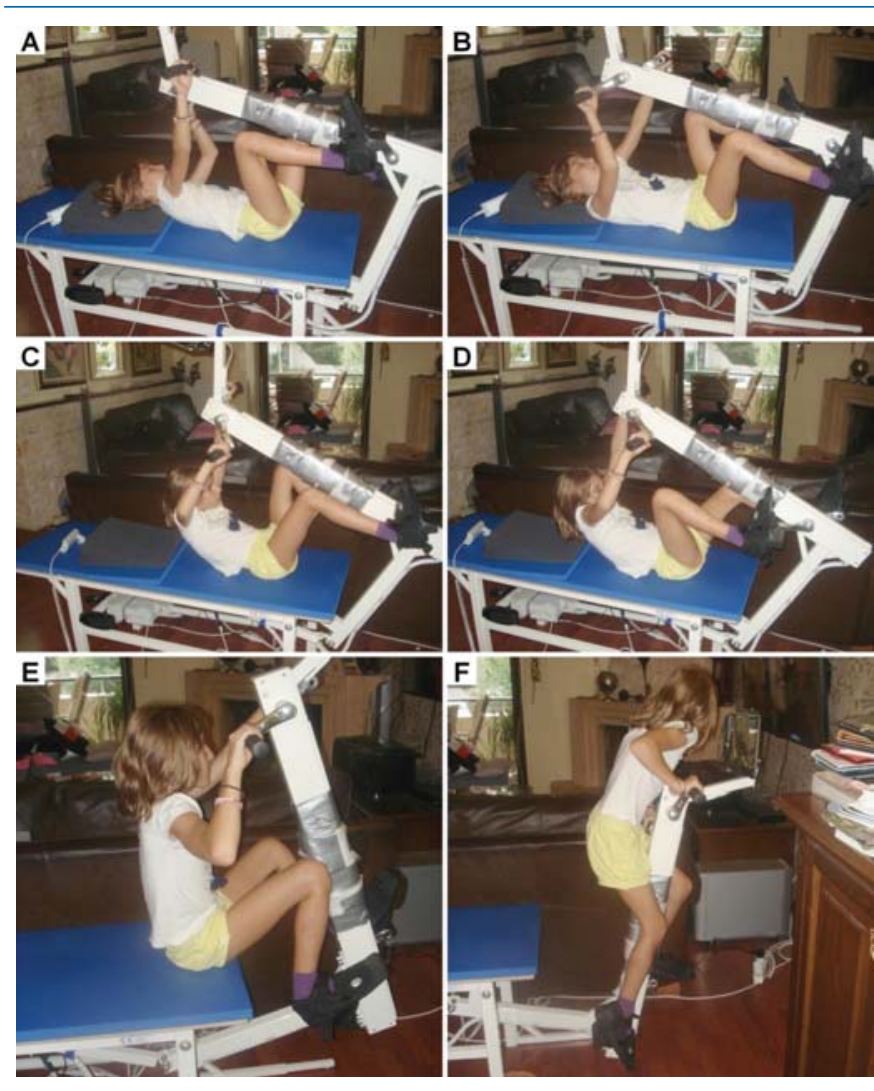

Figure 56: Patient with SCI during exercising on the special CDT device different movement patterns to improve phase and frequency coordination of neuron firing. In A and B also trunk rotation is trained. When turning in the standing position $(\mathrm{F})$, the performance of the right foot is pathologic (plantar flexed).

shows the improvement of CNS organization (lowering of $\mathrm{CD}$ values) when exercising at a load of $20 \mathrm{~N}$. A curve for best values (lowest values) over years of CDT is drawn in Figure 74. One can see from Figure 57 that the CD values varied strongly. There are at least three reasons for the large variation of the CD values. First, her motivation for smoothly exercising varied; she often liked to play and did not concentrate sufficiently. Second, her leg power varied, making a smooth turning more difficult, especially for the difficult coordination's between pace and trot gait. Third, with each bit of reorganization in her CNS, transiently the CNS organization gets worse till it improves again with training. In the older SCI patient Kadri it was measured that with every bit of improvement of her CNS functioning, her coordination dynamics values got transiently worse (Figure 24).

3.3.5. Learning of deep network complexity in the short-term memory by exercising coordinated movements at high load and its problems of induction: To improve CNS functioning, the developing child or the patient has to exercise on the special CDT device in the forward and backward direction to improve symmetries of network organization. By turning fast and slow, the movement performance can be improved via the movement-improving, movement-induced afferent input which is generated by the slow, medium-fast (fast fatigue resistant) and fast contracting muscle fibres (fast fatigue) and their corresponding upstream neural networks including the premotor spinal oscillators (Figure 11).

The exercising against different loads has also to be performed to enhance the integrativity (which means that many subnetworks are activated simultaneously and coordinated) and complexity of neural network organization. By increasing the load during longer periods of exercising, the slow muscle fibres (and the upstream networks) (Figure 11) are trained, which, for example, a marathon runner needs. To get sufficient oxygen, to regulate the temperature and the cardiovascular performance, the vegetative nervous system is trained and improved in its functioning in general and especially because of substantial learning transfer from movements to other untrained functions in the injured and uninjured CNS.

When the CNS functioning of Nefeli had improved sufficiently she was supposed to start regularly the low $(20 \mathrm{~N})$ and high-load $(20 \mathrm{~N}, 50 \mathrm{~N}, 100 \mathrm{~N})$ testing and exercising. She was mentally prepared to start at a certain day that hard exercising. But when the day came, she did not want to do this hard training, because she was in a bad mood, had headache or bowel pain and may be some pain from the re-innervation of the S1 segment (dermatomes and sclerotomes; Figure 58). She was angry with the Author because she did not want to do that test. The father pushed her and struggled with her for one hour. Nefeli's arguments were that she is exhausted and wants to play. She wants to learn for the school so that she is again one of the best ones. But the father succeeded and Nefeli started to turn 1000 times at very low load to improve her nervous system functioning in the short-term memory. Then the $20 \mathrm{~N}$ test for 21 min was started. She was 


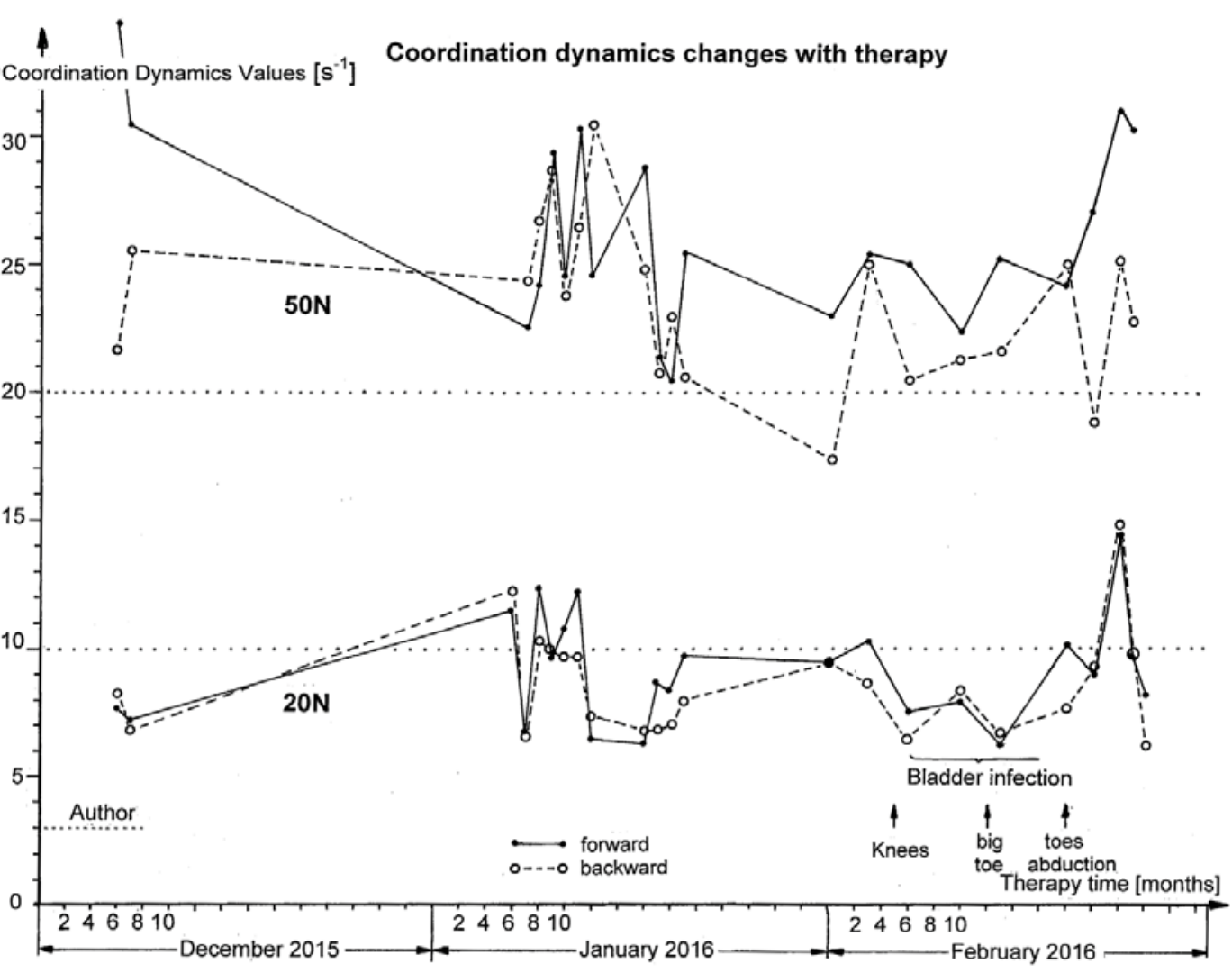

Figure 57: Coordination dynamics values over time of the SCI patient Nefeli.
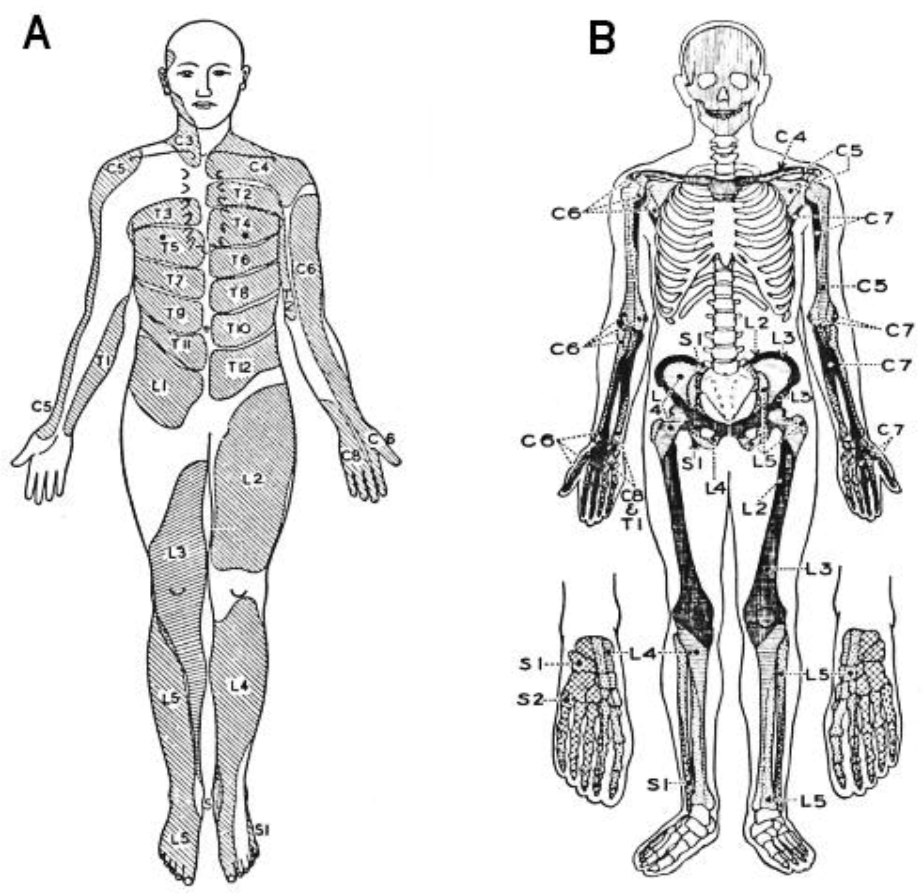

Figure 58: Sclerotomes (B) and Dermatomes (A). 
still in a bad mood. During the $20 \mathrm{~N}$ test her mood improved and she became further cooperative. After a small brake the high-load test was started. Because of the improved nervous system functioning, she started to fight to manage 50 and a bit $100 \mathrm{~N}$. To manage also the $100 \mathrm{~N}$ load is really difficult for a ten-year-old girl with a SCI, because substantial leg power is needed. But after the high-load test she was in a very good mood and had no pain any more. She was now optimistic and wanted to run, walk fast and jump. She took the stop-watch to also measure the running times for quantifying the progress in running. She became able to run first time $13 \mathrm{~m}$ in $16 \mathrm{~s}$ including two times falling. When running that distance a second time, she did not fall and needed now only 10.6s. To run a third time, she refused, because her legs were exhausted (70\% SCI, Th10). Running backwards took much more time. When administering the low-load and high-load testing again, which is anyway a very good exercise, similar motivation problems occurred. Only with a parent present the measurements were successful at the beginning.

When Nefeli had a bladder infection, the motivation problem was bigger, because of reduced power. Bladder infections are quite a load for the body. Even though the body needs the energy to fight against the infection, the exercising at low load is helpful, because the micro-circulation improves and the exercisedependent NK cells (immune cells) are build more, which helps to fight against the infection. A better microcirculation with more immune cells is also successful in cancer treatment $[65,66]$.

Interesting with these low- and high-load testing was, that firstly this exercising at low and high loads changed her feelings completely. Also, the Author feels every day much better after low-load and high-load training up to $150 \mathrm{~N}$ or $200 \mathrm{~N}$. Secondly, even though being a bit exhausted, the performance of movements was much better after exercising against high load. This holds also for healthy pupils and athletes. The improvement of CNS functioning in the short-term memory has consequences for the improvement of movements and probably also for the health in general. The improvement of the health in general was important for Nefeli. After the cancer removal no chemo and radiation therapy were administered because of the SCI. There was therefore a bigger risk of a re-occurrence of the cancer. Exercising helps to inhibit a re-growing [66].

3.3.6. Improvement of free running: After 9 months of CDT the running was tried and sometimes Nefeli got into the running rhythm for a few steps. But after approximately 10 months she became really able to run a bit with poor performance. Nefeli was always open to run, unless the power in the legs did not allow it anymore because she wanted to run again with the other children at school and she liked the running very much. Running is a very integrative movement and trains deep neural networks complexity efficiently. Pathologic leg activation is partly compensated for by the kinetic energies which smoothen the movements. The stimulation of leg movements via arm and hand movements was limited in her because of balance problems, which made her to abduct the arms. To stop the falling due to losing the balance was solved in the way by letting her running along a floor (Figure 59), where she could touch the walls for keeping the balance in similarity to the running on treadmill where she could touch the rails for keeping the balance. But she was also falling because of the low stability of the running pattern. The potential well of the running pattern was too shallow and the phase and frequency variability too high. But as mentioned above, Nefeli was not afraid of falling and had developed a good technique to reduce the severance of falling and to get quickly up again (Figure 55).

The pattern stability of free walking improved because the walking could be trained for longer distances.

The improvement of running is shown in Figure 59. The backward running also improved. At the beginning of running, she could faster walk than run. But with the improvement of the running performance she was faster with running than with walking. Measuring the running times, plotting them and showing them to her motivated her to train more. She wanted to be better than the last times.

3.3.7. Using the stepping automatism for improving walking and running: One problem in repair was how can Nefeli made to lift the knees and strike the ground first with the heels to improve the performance of walking. 

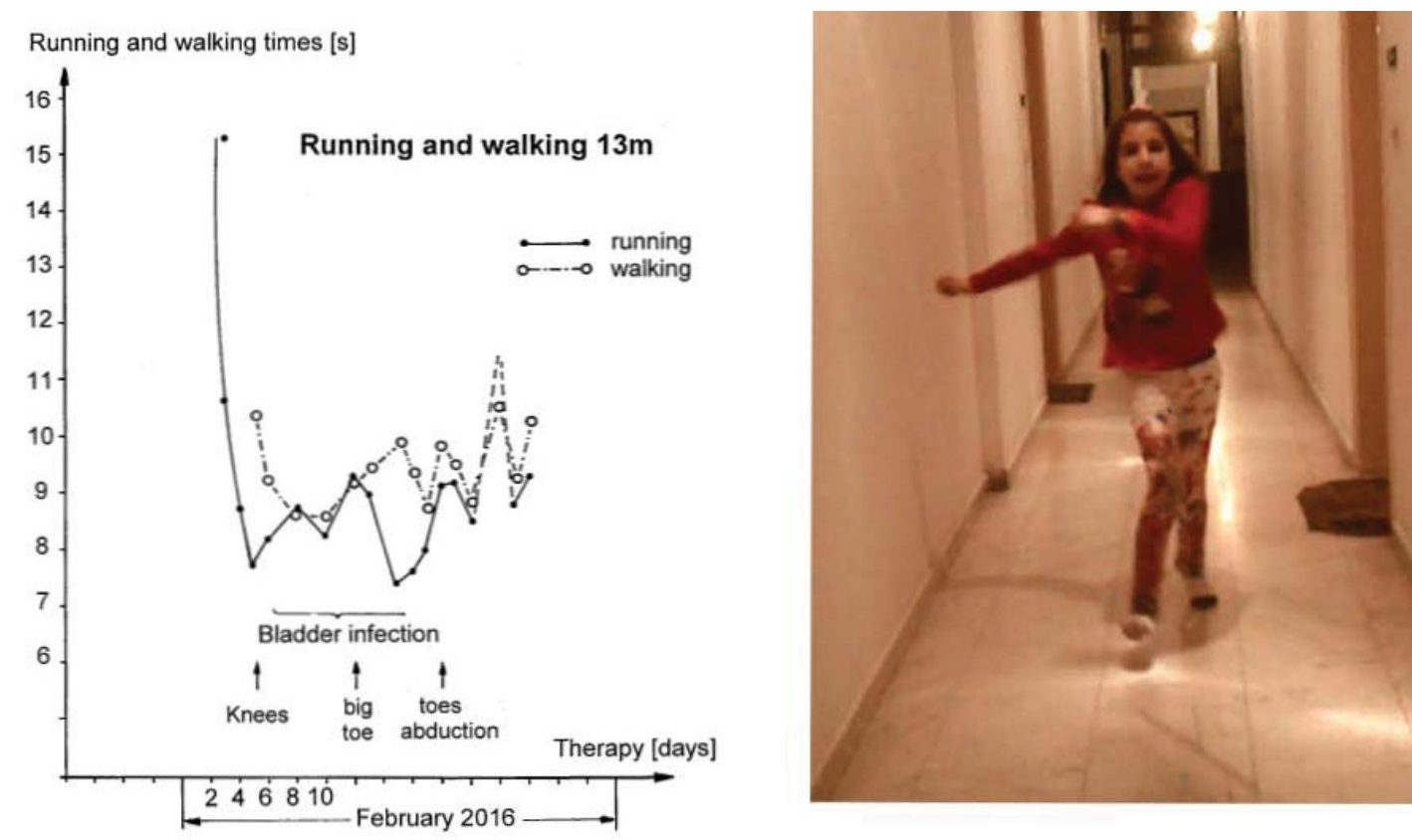

Figure 59: Improvement of running and walking times when moving along a floor. The patient is using the arms also for keeping the balance.

In brain injury the brain has to be repaired. In SCI (Nefeli) the brain has to be made reorganizing the problem to compensate for the injury and mismatch of nerve/tract fiber growing. For a mainly functional repair, the remained tract fibers below the injury level have to be 'rewired' by the brain, because there is only little plasticity in the intrinsic networks of the spinal cord. During a regeneration of the caudal spinal cord, the growing nerve/tract fibers may not find the to be re-innervated muscles/organs because of mismatch. Again, the brain has to compensate for the false growing in the periphery. The complexity of repair mechanisms is large. But through movement-based learning, the brain is able to take care of the complexity of CNS repair. In lower animals it is or was believed that the power of regeneration is very high and the growing nerve fibers know the place where to grow to. In human, the regenerative capacity is small; but the tremendous complex brain is able to solve the problem by learning. One strategy for re-learning is here the used stepping automatism.

Characteristic for the stepping automatism in babies is the high lifting of the knees (Figure 53). One stimulus for inducing the stepping automatism is the heel strike. If it would be possible to (partly) stimulate the stepping automatism for repair, then, may be, the neural networks at the SCI site (around Th10) could be stimulated for functional repair and regeneration. Since the neural networks of the stepping automatism are mainly located in the intumescentia lumbosacralis and are getting only little drive from supraspinal centers, the stepping automatism pattern should be quite physiologic in a Th10 SCI and could help to improve the feet functions during walking.

After 11 months of therapy Nefeli became able to lift the knees sufficiently and became able to strike the ground first with the heels to induce the stepping automatism (Figure 60). Because the sensitivity of the soles of the feet had improved, she could feel that the heel was striking the ground first (before the forefoot). To optimally use the movement-induced afferent input for walking and running, Nefeli was walking and running best without shoes.

3.3.8. Training of the damaged spinal cord site: The devastating consequence of a SCI is that the functions below the injury level cannot be controlled any more by supraspinal centers. But the neural 


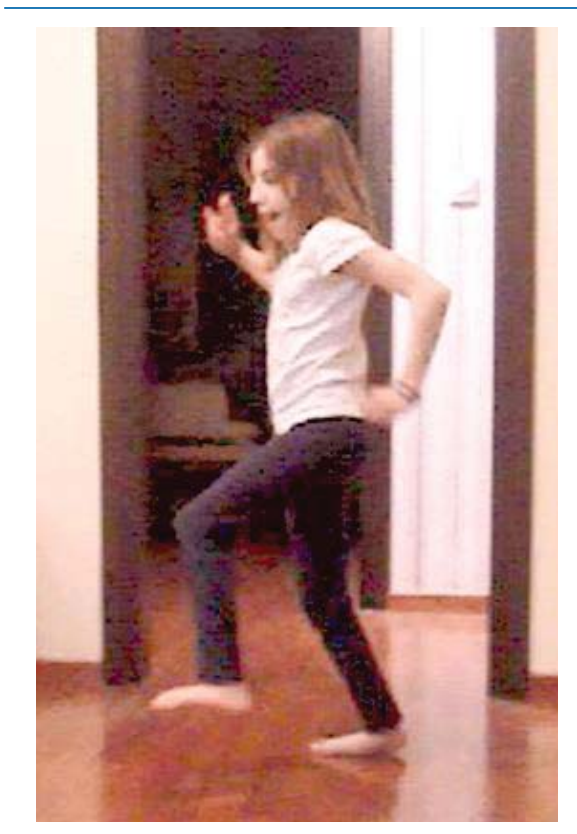

Figure 60: The patient with a SCI learned to lift the knees and strike the ground first with the heel to induce the stepping automatism for SCI repair. Note, the arch of the foot is preserved in the left foot but not in the right foot during the stance phase. With the lifting of the knees, Nefeli became able to activate the quadriceps much more; the regeneration of the spinal cord had substantially reached the L4 segment. Nefeli is walking in the usual trot gait coordination. networks at the injury site, in Nefeli's case Th10/11, are additionally damaged and partly lost. Nefeli trained the trunk stability including the Th10/11 spinal segments, when exercising on the special CDT device in the lying position with trunk rotation (Figure 56A,B).

The teachers and pupils of the school were following up Nefeli's progress, induced by CDT, from the beginning on when she was moving with sticks (Figures 32B,46A). The teachers tried to contribute to the progress. The sport teacher gave Nefeli a hula hoop for training the trunk and especially the pelvis control (Figure 61). The progress of trunk movements was quantified by the time she could hold the hula hoop up.

3.3.9. Re-appearance of big toe function in relation to changes of the homunculus of the sensory-motor cortex: After 10 months of CDT, Nefeli became able to move the forefoot and a bit the toes altogether (Figure 54). With 10.5 months of CDT she became able again to move a bit the big toes separately (Figure 62). The dorsal flexion was easier to perform than the plantar flexion. She was glad about this new movement, because she was already afraid that she forgot that movement and could not activate it any more. The visualization of the task and movement was very important to initiate, control and continue the (slow) movement.

Unclear is whether she only forgot the initiate that toe movement pattern or whether in the sensory-motor cortex (Figure 63) the adjacent parts took these neural networks partly over and used them for other functions. Interesting is actually what changes of the homunculus take place after complete and incomplete SCI.

There is also localization of function in the cerebral cortex. To substitute function from one part of the CNS to another, it is important that many brain parts of a certain function are activated simultaneously and in a coordinated manner. Probably especially neighboring brain areas can take more easily functions over. Since foot, bladder and sexual functions are close together in the sensory-motor cortex (Figure 63), the repair of foot functions is not only important for re-learning walking and running, but also for the repair of bladder and sexual functions.
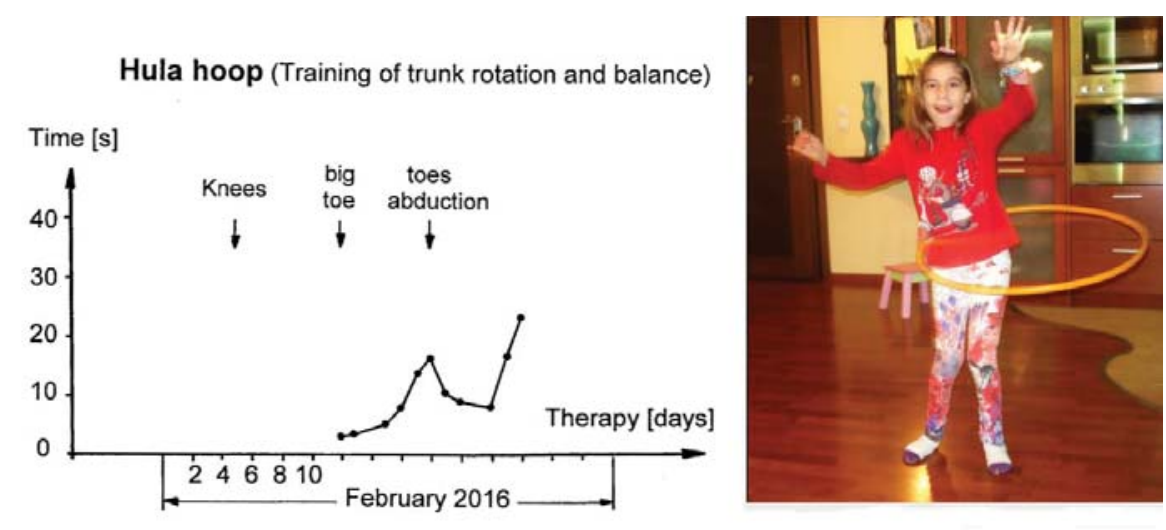

Figure 61: The 10-year-old SCI patient Nefeli during training with the hula hoop to improve trunk mobility/stability and balance. 

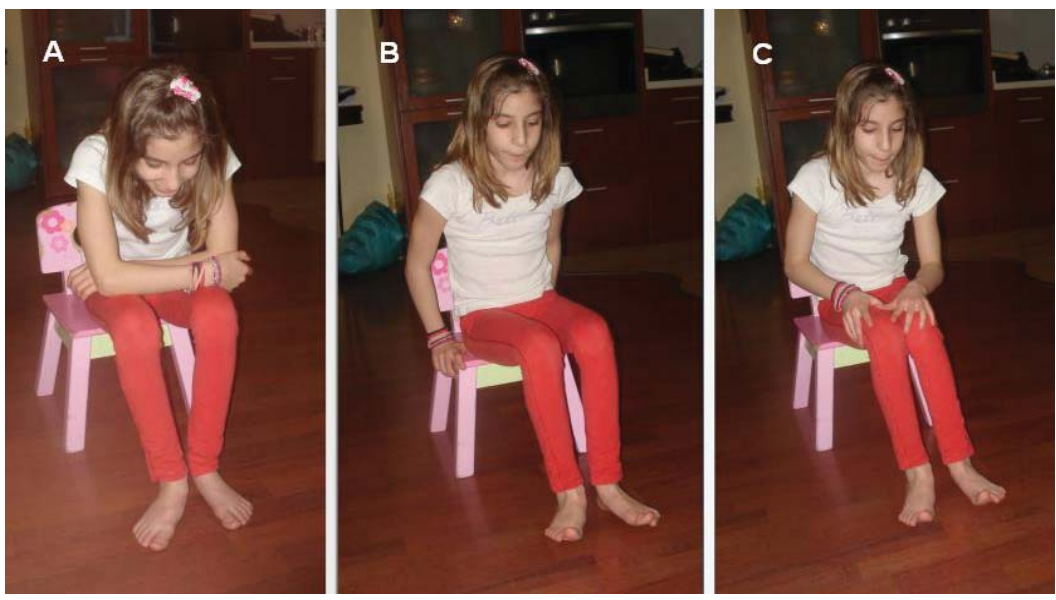

Figure 62: Ten-year-old Nefeli can move again the big toe separately four years after the accident and following 10.5 months of CDT. In A she does not activate foot muscles and is relaxed. In B,C she concentrates and visualizes the task to activate the dorsal flexion of the big toe, which indicates that the spinal cord repair has reached with the activation of the halluces longus the spinal cord segment L5.

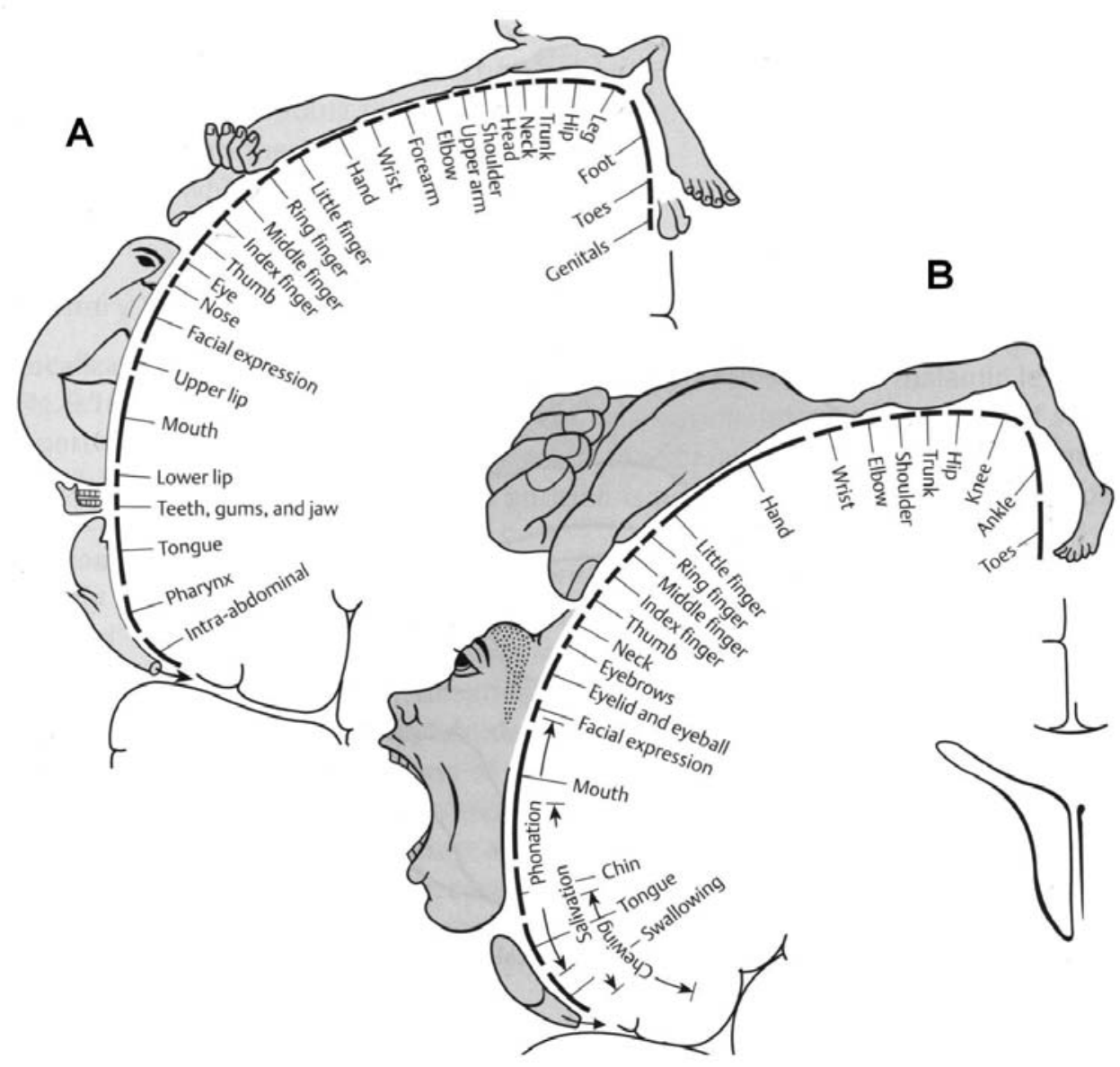

Figure 63: Relative sizes of the cortical representations of different parts of the body in the human primary somatosensory (A) and motor (B) cortical fields (the so-called homunculus). (from: Penfield W and Rasmussen T: The Cerebral Cortex of Man, Macmillan, New York, 1950). Note the large representations of fingers, hand, mouth, lips, tongue and phonation. The much larger representation of the index finger than the upper trunk is in accordance with the corresponding two-point discriminations $(1 \mathrm{~mm}$ against $25 \mathrm{~mm}$ ). Following incomplete $\mathrm{SCI}$ and repair, these cortical representations change. 
In the patient Nefeli, spinal cord tract fibers had to be rewired by changing partly localizations of functions in the brain. Walking, bladder and bowel functions have to be repaired and colon pain stopped by shifting, enlarging, lessen and combining cortical representations of functions.

3.3.10. Repair of rectum und colon functions: Emptying the rectum is similar to emptying the urinary bladder [17]. Filling of the rectum activates stretch, tension, mucosal and under certain circumstances pain receptors in the rectal wall (and may be some skin receptors around the anus), which transmit impulse patterns by way of the inferior hypogastric plexus and sacral roots to segments S2 through S5 of the sacral spinal cord, where probably a defecation center is located in similarity to the micturition center in the sacral spinal cord. The sacral defecation center communicates probably with a pontine defecation center in the reticular formation and the cerebral cortex.

Rectal peristalsis is induced by parasympathetic activation from the defecation center, which also induces relaxation of the internal sphincter in similarity to micturition. The sympathetic nervous system inhibits peristalsis. The external sphincter consists of striated muscle fibers of FR type, innervated by $\alpha_{2}$ motoneurons (Figure 11), and is under volitional control. The fast fatigue resistant muscle fibers secure that the sphincter can be contracted quickly and the closure can be hold for longer times. Rectal emptying is mainly accomplished voluntary by abdominal pressing.

The stretch, tension and mucosal receptors of the rectum are similar to those of the bladder and their activity was recorded when stretching the external anal sphincter by pulling the anal catheter and changing the catheter thickness from thin to thick (Figure 8).

The Author had given research emphasis to the repair of the urinary bladder functions because of the bladder infections and the risk of ascending infections. Kidneys cannot be transplanted to those patients because of the necessary suppression of the immune system after transplantation. It was difficult to administer movement therapy to Nefeli when she had pain. Pain is a general problem in SCI. Even though CDT inhibits most likely cancer growing, the differential diagnoses of pain includes besides infections of kidneys and ovaries the growing of metastasis in the bowel.

Transection of the spinal cord above the lumbosacral center for defecation leads to fecal retention. Overfilling of hollow viscus is perceived as pain. In incomplete SCI, the pain can be felt. There are SCI patients which are afraid of a repair of the sensitivity because of the possibility of getting untreatable pain.

Since the middle and left abdomen was sensitive to touch and pain, Nefeli could feel the pain in the Heads zones for bladder and rectum/colon (Figure 64). Nefeli felt pain in the Heads zone for the bladder with bladder/urethra problems and she nearly every day felt pain in the Heads zone for the rectum/colon in the morning and after eating. When exercising for approximately 30min on the special CDT device the pain mostly waned. Her bowel/colon/rectum pain most likely occurred due to the impaired peristalsis, caused by the impaired function of the parasympathetic nervous system, due to the SCI. The exercising on the special CDT device activated the peristalsis of the bowel by activating the parasympathetic division and the pain disappeared. The exercising on the special CDT device therefore does not only activate in the shortterm memory the bladder for micturition but also the colon/rectum for defecation.

3.3.11. Pain perception and Zones of Head: It can be expected that Nefeli experienced pain in the lower body because of the incomplete SCI Th10. When the lower body started step by step to function again, pain occurred in the toes, knees, bowel and other parts of the lower body. To distinguish between pain she had to accept (because of mobilizing the body again due to therapy) and dangerous pain, also the heads zones (Figure 64) had to be taken into consideration. An explanation of the zones of Head is the following.

The cell bodies of the afferent autonomic fibers, like those of the somatic afferent fibers, are located in the spinal ganglia. The autonomic fibers enter the spinal cord through the posterior root together with the somatic afferent fibers from the myotome and dermatome of each segmental level. Thus, each individual segment of the posterior horn receives converging afferent input, both from internal organs and from the 
related myotome and dermatome (Figure 64A). Activation from either set of afferent fibers (visceral or somatic) is transmitted centrally by the fibers of the anterolateral spinothalamic tract (Figure 64A). It is therefore understandable that pain arising in a particular viscus (bladder, colon) is sometimes felt elsewhere, namely in the dermatome or myotome represented by the same spinal segment. This phenomenon is called referred pain. It may be accompanied by a certain degree of hypersensitivity to somatosensory stimulation in the dermatome to which the pain is referred. The abdominal wall may also become rigid. It is also possible that impulses arising from the skin can be projected (referred) to the internal organs. Clearly, the somatic afferent fibers are interconnected with visceral reflex arcs within the spinal cord. This may explain how therapeutic measures at the body surface (such as the application of warmth or heat, compresses, rubbing, etc.) often relieve pain arising from the autonomically innervated viscera.

In the patient Nefeli with an incomplete SCI at the level of Th10 we can expect pain origination in the small intestine, colon, kidney, ovaries and urinary bladder (Figure 64B). Especially we have to expect pain in the colon zone of Head (Th11, Figure 64B), because at this level there is the cord injury. And indeed, it seemed that she experienced pain in all of these zones of Head. Often Nefeli experienced pain in the colon zone already after sleeping in the morning. But when jumping on springboard, running or doing hula hoop after some time she often got pain in the colon zone. During jumping and running the bowel, including the colon, will move up and down and stimulate visceral receptors of the colon. On the other hand, pain in the colon zone, due to performing hula hoop, may arise because the SCI site is stimulated and may arise not so much from the mechanical stimulation of the bowel. Even though the PET showed no tumor growth, still small metastasis may escape detection and grew anywhere in the intestine and could give rise to pain later on. An important property of CDT is therefore that tumor growth will be inhibited simultaneously to a certain extent anywhere in the body. So far Nefeli refused healthy nutrition which stimulates the peristaltic of the bowel.

Additionally, Nefeli had pain in the urethra immediately following micturition. Such pain may be due to a hypersensitivity of the urethra wall and the skin around the vagina arising with the re-innervation of the urinary bladder. Hypersensitivity of the skin often occurs with re-innervation.

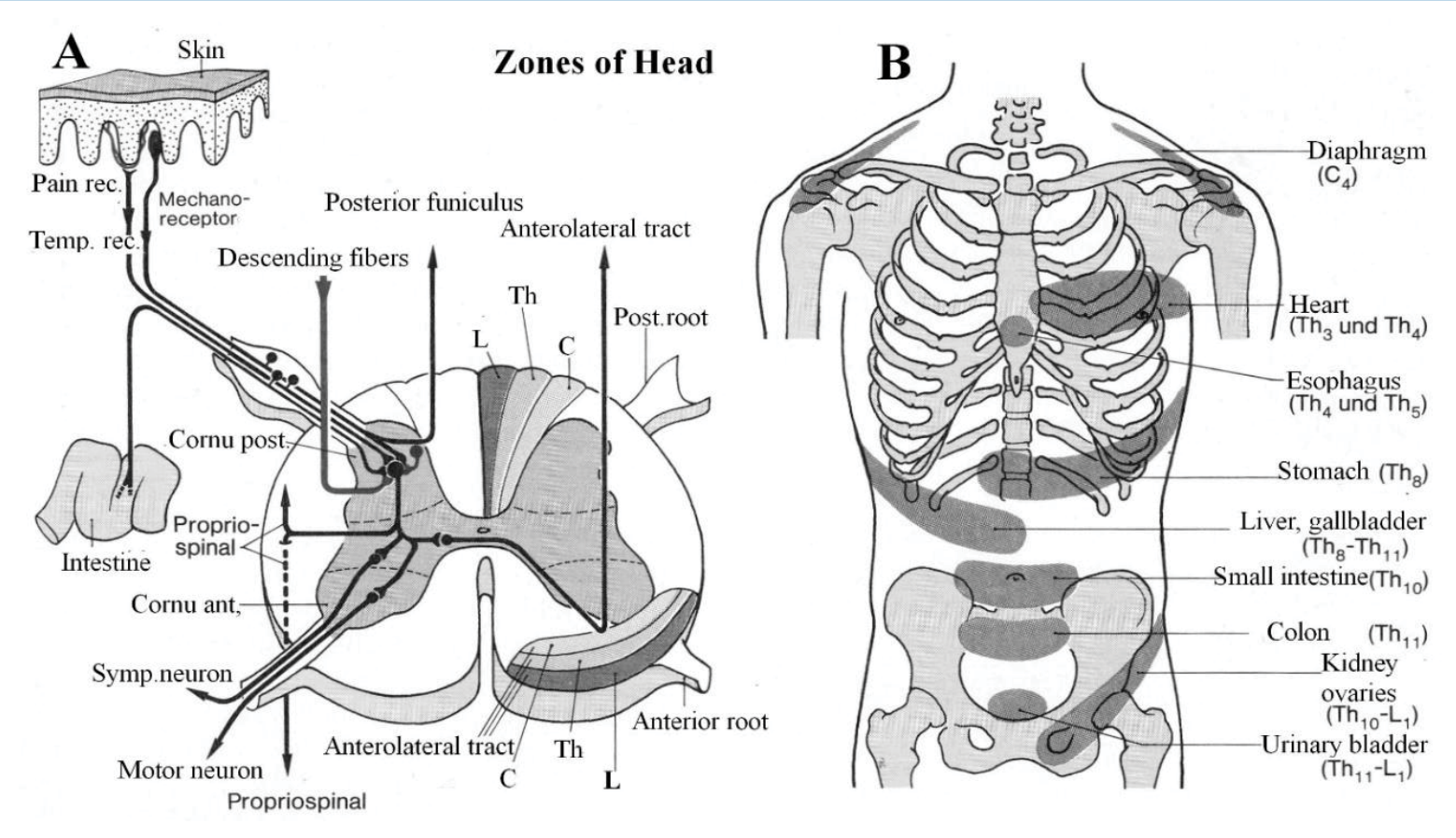

Figure 64: Zones of Head and referred pain. 
When pain occurred during exercising, the training of that certain movement was stopped and another movement was trained or a small break was taken intermediately till the pain was over.

3.3.12. Waning of the regeneration of Nefeli's spinal cord: Above it was shown that the Nefeli's spinal cord regenerated. The spinal cord regeneration was measured by the recurrence of function of segmentindicating muscles (Figure 37). But the regeneration waned after one year of therapy. The important question is why did the regeneration of the spinal cord faded? One reason is that Nefeli stopped training continuously at the limit, against the advice of the Author. The chain of events for keeping certain regeneration processes going was broken with every therapy break. A continuous therapy at the limit would probably be needed for a year or more to run through the whole chain of events for continuous regeneration. Another important reason for the waning of the regeneration is that the regenerative power is probably much stronger below an age of 10 years. Also, little is known about the precise damage at the injury site. Further, the limited regeneration of her spinal cord was probably supported by recruiting ectopic pathways through root connections, sympathetic chain or plexus connections (misdirected haphazardly during development) or a re-organization of plexuses outside the spinal cord. But the regeneration speed of $1 \mathrm{~mm} /$ day supports the direct growing of nerve fibers caudally in the spinal cord.

The first two reasons for the waning of the regeneration process have important clinical consequences. If the regenerative capacity is much stronger below an age of 10 years, then children have a much better prognosis with respect to repair than teenagers or adults. From the genetic point of view, it would be important to find out what are the differences in gene expression below 10 years of age and adults and can we change in adults the gene expression to the one below an age of 10? What are the differences of interaction of neural activity and genetic programs during development and repair?

The second possibility that one has to run through the whole chain of events to stimulate all possibilities of repair has the clinical consequence that longer therapy times are needed when administering efficient therapy. The treatment possibility that one has to run through the whole chain of events is supported by the success of bringing a permanent coma patient back to life. With four years of continuous CDT with 20 hours therapy per week, the coma patient became conscious again and with 6 years of continuous therapy he could speak again [67].

3.3.13. Gene expression pattern triggered by excitation in proliferating adult NPCs: For weight changes of synapses, growing of axons and dendrites, neurogenesis and building of glia cells during the process of activity induced structural repair, proteins are needed, which have to be generated by appropriate transcription from genes. It seems that natural patterned activity-dependent depolarization of the plasma membrane trigger activity-dependent gene expression programs [68]. $\mathrm{Ca}^{2+}$ signaling through $\mathrm{Ca}_{\mathrm{v}} 1.2 / 1.3$ channels and NMDA receptors can activate a broad array of rapidly responsive transcription factors. Moreover, excitation could in principle induce release of autocrine factors from the NPCs themselves, leading to recruitment of a host of additional signaling pathways to nuclear transcription factors [68].

3.3.14. Regulation of epigenetic modification for repair by movement-based learning: To generate repair in the nervous system, it is likely that permanent changes in gene expression patterns are achieved through permanent changes in chromatin remodeling without changes in DNA sequence. The concept of chromatin remodeling addresses a key challenge of how stable changes in gene expression are induced [69] in neural networks to produce long-lasting changes in repair. DNA methylation is one of the many epigenetic modifications that can alter gene expression. Dynamic and reversible DNA methylation may be essential for learning and memory formation and could transmit repair influences onto adult neurogenesis.

If the epigenetic definition that the events are the structural adaptation of chromosomal regions so as to register, signal or perpetuate altered activity states [70], that means the nature of epigenetics is responsiveness, then epigenetic regulation for repair should be stimulated by physiologic neural network activation. Un-physiologic neural network activation like electro stimulation (which is nearly always unphysiologic apart from the heart pace maker) stimulates chromosomal changes in a negative direction with 
respect to health. "The DNA methylation system and the Polycomb/Trithorax systems seem to respond to previous switches in gene activity" [70].

Understanding the complex epigenetic regulation of neural activity and adult neurogenesis is integral to designing therapeutic approaches to restore neurogenesis and cognitive functions. It will also give a tremendous insight into understanding how certain environmental or pathological influences, such as stress, physical activity, depression and epilepsy regulate adult neurogenesis [71]. Figure 65 shows steps of epigenetic regulation induced by specific physical activity, namely movement-based learning.

Research in movement-based learning has to identify how epigenetic mechanisms can be efficiently modified by the performance of specific, corresponding movements or learning processes to improve development ("correction en route", which is particularly salient to the treatment of cerebral palsy) and repair of the human CNS and to avoid pathologic CNS changes like epilepsy and cancer. The complexity of the epigenetic regulation (Figure 65) is tremendous. Already the neural network learning for repair is complex and needs human neurophysiology including new measuring methods.

3.3.15. Spasticity and the intrinsic neuronal apparatus of the spinal cord: Patients with a SCI or a brain injury can have little to very strong spasticity depending on the kind and severance of the injury. Here it is concentrated on the spasticity caused by a SCI.

Spinal cord spasticity is a pathologic pattern of the neuronal networks of the spinal cord. In clinics spasticity is reduced by the administration of spasmolytic drugs. The drawing back of spasmolytic drugs is

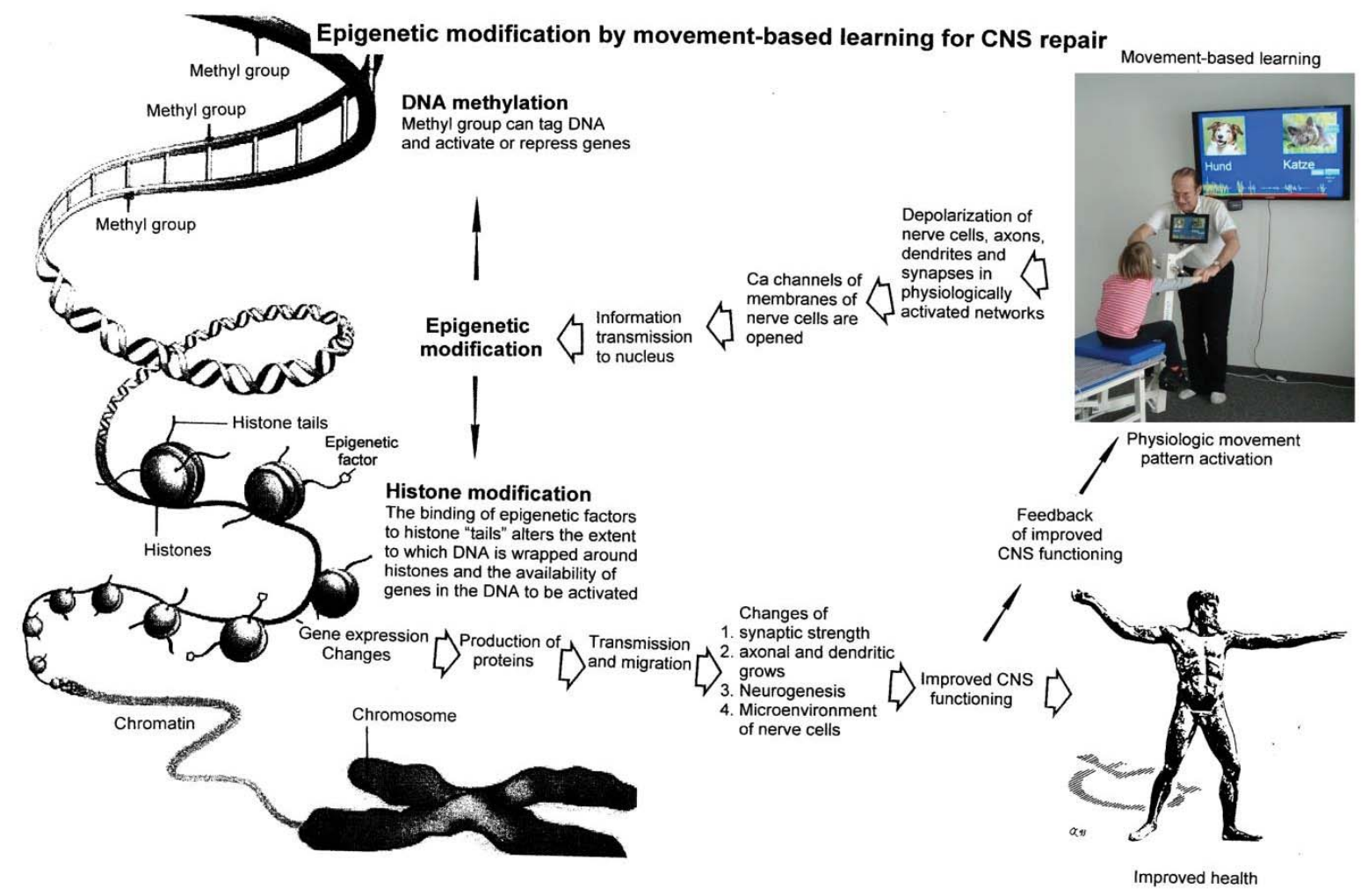

Figure 65: Epigenetic regulation for repair by movement-based learning. CDT-induced stimulation of the pathways that regulate neural network repair is a proven therapeutic and preventive tool. Epigenetic mechanisms, stimulated by physiologic network activation, are likely key players within signaling networks, as DNA methylation, chromatin remodeling and small non-coding RNAs superfamily are required for the fine-tuning and coordination of gene expression during neural network repair by learning. Since the nervous system is involved in nearly all body functions, CDT will improve health. 
that they do not only reduce the spasticity but also the power of the volitional movements. Also, there are substantial side effects.

The patient Nefeli with an incomplete spinal cord at the level of Th10 had strong extensor spasticity and some flexor spasticity besides rigor. When she was standing at the whiteboard at school and wrote there (Figure 32C), here standing was not fully physiologic. Her knees were too much flexed. Probably extensor and flexor spasticity were a bit at work. On volition she could stay straight, but as she said, she would need a lot of power for that. If spasmolytic drugs would be administered to her, the spasticity would reduce, but she would have more problems with walking, because of the reduced power in the legs. The proper way to handle spasticity is to reduce the strength of the spasticity and increase the strength of physiologic movements through CDT. Within the System Theory of Pattern Formation, the potential well of spasticity has to be made shallower and the potential wells of physiologic patterns deeper (Figure 66). This can be achieved to a certain extent by movement-based learning. Physiologic movement patterns are trained which are away from the pathologic spasticity patterns (extensor and flexor spasticity) so that not also the spasticity patterns are trained.

When the Author was holding Nefeli (Figure 67A), she showed strong extensor spasticity. When she was lying on the special device at the beginning of training, it seemed sometimes impossible to flex her knees. But on volition she is able to flex the knees to be fixed to the special CDT device for exercising or to walk. The extensor spasticity may be mainly related to walking or running which are human specific up-righted movements. Anyway, spinal cord spasticity is generated by the damaged neural networks of the intrinsic apparatus of the spinal cord. This spinal cord apparatus can generate complex movements.

When a finger touches a hot stove, the hand is pulled back with lightning speed, before any pain is felt. The action potentials stimulated in the cutaneous touch receptors and nociceptors travel by a wave of patterns in many skin afferent fibers to the substantia gelatinosa of the spinal cord to activate this safety automatism to protect the skin. Figure 10 shows such impulse patterns conducted in skin afferent fibers.

\section{Improvement of the coordination dynamics in the short-term memory}

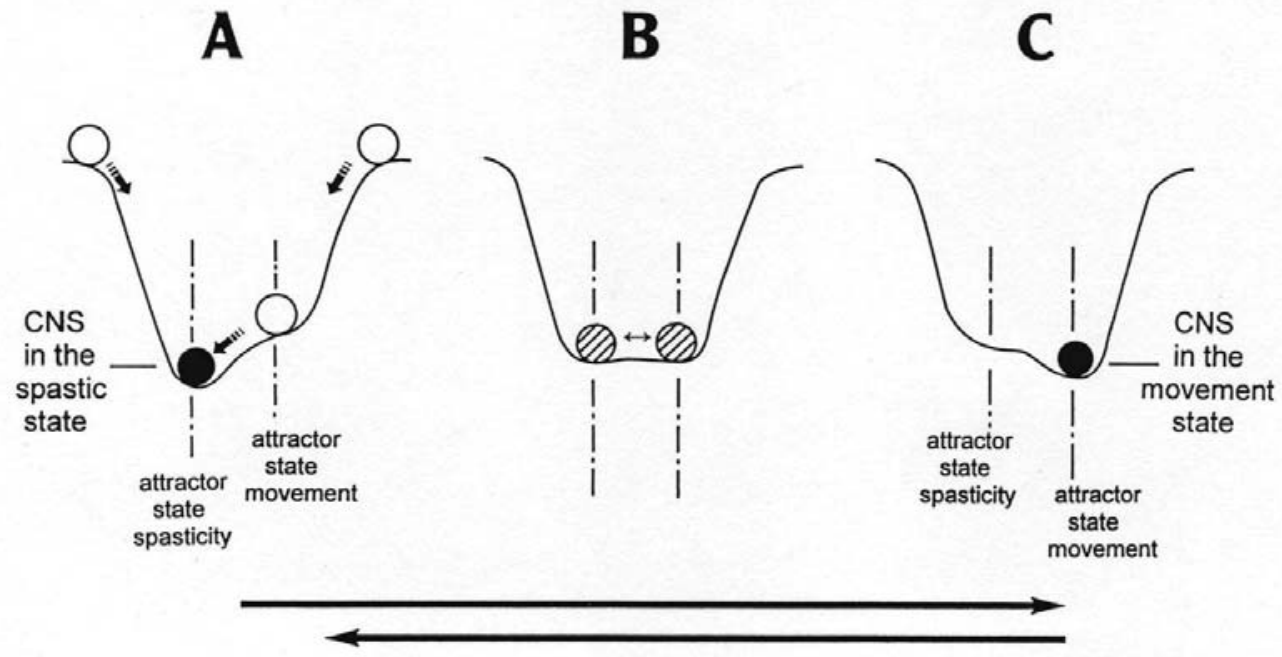

Figure 66: Therapy-induced spasticity reduction in the short-term memory. The position of the ball represents the state of the system and the potential well, the attractor. The ball is attracted to the stable position in the deepness of the hole, called attractor state. The attractor layout, consisting of two attractive states of different stability, is changing upon exercising very coordinated rhythmic movements. Black ball = stable state, open ball $=$ very unstable state, hatched ball $=$ spasticity and movement co-exist. Variability of phase and frequency coordination not indicated. 


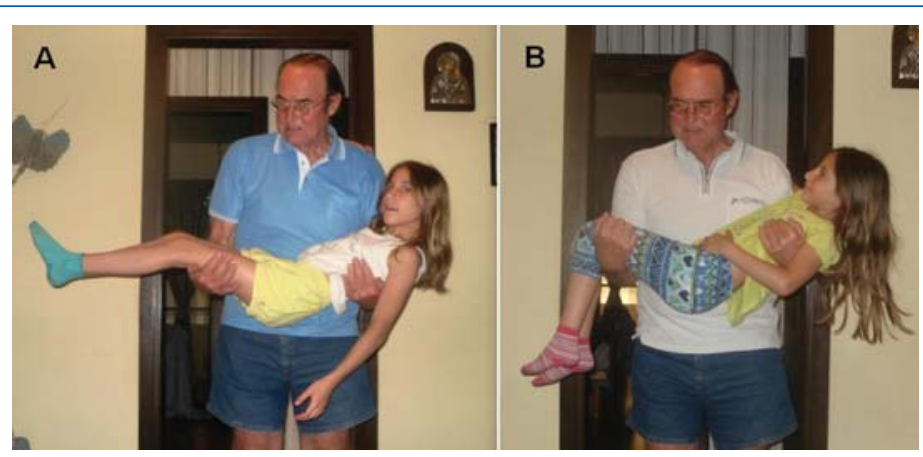

Figure 67: Practical judgment of extensor spasticity. When the Author holds the patient Nefeli (SCI Th10), one can judge her extensor spasticity by the extension of her legs (A). Her present spasticity is medium strong. A physiologic leg position can be seen in B. The healthy seven-year-old sister has no spasticity and the legs are flexed.
The fastest pain fibers (P in Figure 10F, pin-prick 1) will contribute substantially, because fast conduction of pain afferent fibers is needed for safety. According to Figure 9 the fastest pain fibers conduct with $13 \mathrm{~m} / \mathrm{s}$ at $36^{\circ} \mathrm{C}$. The distance from the finger to the spinal cord is approximately $0.8 \mathrm{~m}$. With $\mathrm{t}=\mathrm{s} / \mathrm{v}=$ $0.8 \mathrm{~m} / 13 \mathrm{~m} / \mathrm{s} \approx 0.06 \mathrm{~s}$, the conduction time in the fastest pain fibers from the finger to the spinal cord is $0.06 \mathrm{~s}$. Some further time is lost in the receptors to generate the pattern. Therefore, in approximately $0.1 \mathrm{~s}$ the afferent impulse patterns reach the spinal cord for generating the pattern to pull the hand away. In Figure $10 \mathrm{~F}$ it can be seen that the fastest pain fiber reached the recording electrodes with a delay of $0.1 \mathrm{~s}$ following pin-prick 1. Both values are compatible. The time needed to generate the protection reaction is $0.1 \mathrm{~s}$ (page 343 of [18]). According to this approximate calculation, the hand moves away from the hot stove after $0.2 \mathrm{~s}$. The intrinsic neural apparatus consists of interneurons, association neurons, commissural neurons and the fasciculus proprius around the gray matter of the cord.

The stepping automatism of the newborn baby (Figure 53) is also mainly generated in the neural networks of the spinal cord. One of the stimuli to activate the stepping automatism is the heel strike.

Following brain death diagnosis, the rest functions of the human body are mostly soon lost, including the functions of the spinal cord. But in exceptions in brain-dead humans, when ventilated, the spinal cord is reorganizing itself and is taking body functions over like temperature regulation. It has happened that relations visited a brain-dead human. When they touched the out of the bed hanging leg, the brain-dead human moved via a spinal cord pattern the leg into the bed. Obviously, the intrinsic apparatus of the spinal cord is really a neural network which can generate complicated movement patterns by itself.

Following SCI, the damaged networks generate pathologic movement pattern including spasticity. During CDT the brain learns to activate the damaged spinal cord in a way to generate physiologic patterns again. But the main plasticity is sited in the brain and not in the networks of the spinal cord.

3.3.16. Repair of the foot arch: From Figure 68A it can be seen that the right foot of Nefeli had lost the arch in the stance phase. A foot arch support is only a mechanical help to stop foot arch degeneration. A causal

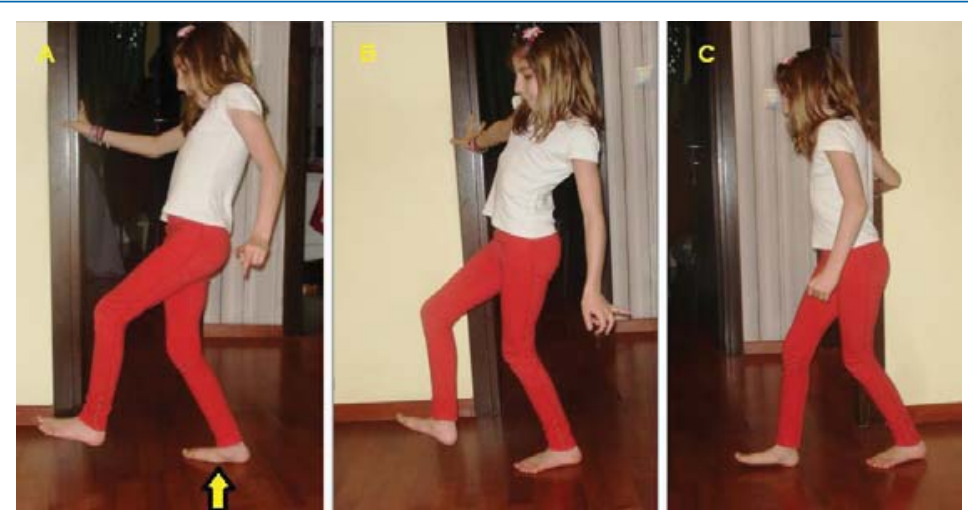

Figure 68: Foot arches during supported walking. Note the right foot has in the stance phase (A, arrow) no foot arch. repair, that means a reconstruction of the right foot arch, can be achieved if the nervous system is repaired. The neural network pattern, generating the foot arch, has to activate the motoneurons which drive the muscle for generating the foot arch. The muscles which have to be brought under control by the walking pattern are at least the Mm abductor halluces, flexor digitorum brevis and quadratus plantae. If the foot muscles are not properly activated, pain will occur later on and orthopedic operations are necessary. 
The movement patterns which activate the foot arches are especially the jumping on springboard in anti-phase (Figure 51) and the walking on forefoot if possible. In Figure 51, the Author is trying with the small finger to reduce the outward rotation of the right foot to improve the movement-induced afferent input for better motor learning.

3.3.17. Participation in school sport: Following 14 months of therapy, Nefeli became able to participate in school sport (fourth class). With some help of the sport teacher she could manage to dance with the other

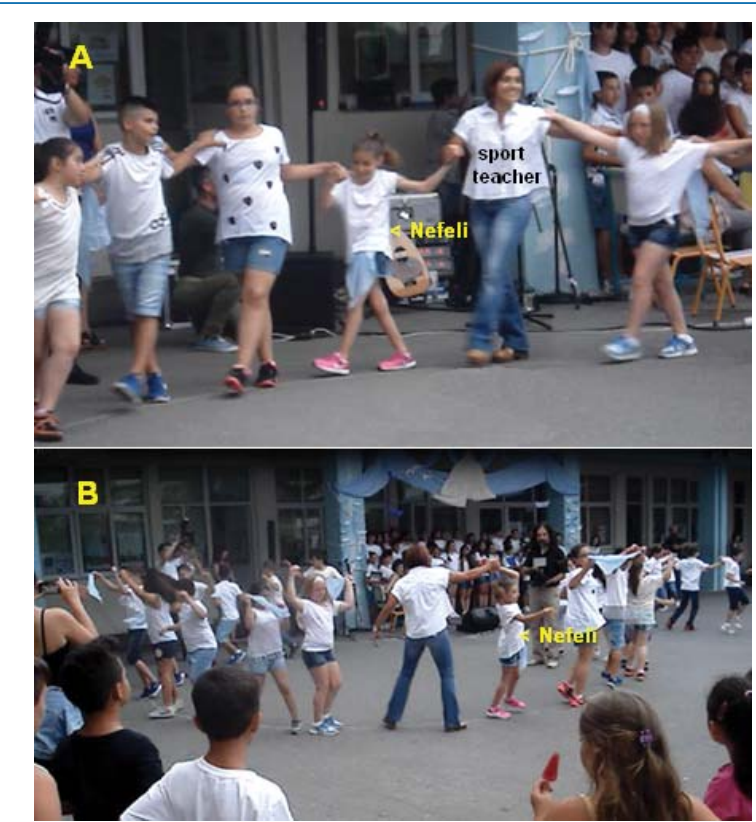

Figure 69: Nefeli during dancing at school. Disappointing is that 16 of the 18 pupils had over-weight.

class maids (Figure 69A). Only the fast rotation movements were difficult for her (Figure 69B). The teachers made a good job to integrate her in all the activities. Nefeli would have suffered if she could not do the same things as the other ones.

3.3.18. Improvement of trunk stability: Due the SCI and the removal of the cancer including the Th10 ganglion, the patient Nefeli had severe scoliosis, a lack of trunk stability and trunk spasticity. Figure 70 shows the false positioning of the trunk in comparison to the healthy brother.

The too much backward positioning of the pelvis could also be seen during walking on the knees. After about 15 knee steps, Nefeli's spasticity of the trunk pulled her pelvis backward and she fell forwardly.

To enhance the trunk repair, also the hula hoop movement was used. At the beginning the movement was difficult for her (Figure 61). But then she could do the hula hoop movement much longer (Figure 71). The limiting factor of the performance time was the balance of standing and bowel pain. As a consequence of the hula hoop movement and other movements like training on the special CDT device in the lying position (Figure 56), her trunk functions improved so that her trunk became straighter during hula hoop (Figure 72) with therapy.

An improvement of a straighter standing can be seen when Nefeli was working in the kitchen in comparison to a standing at the whiteboard at school (Figure 73). The improvement in natural straight standing during further six months of therapy is not big but visible. On volition Nefeli could stand straighter, but that would cost her too much energy for a longer time.

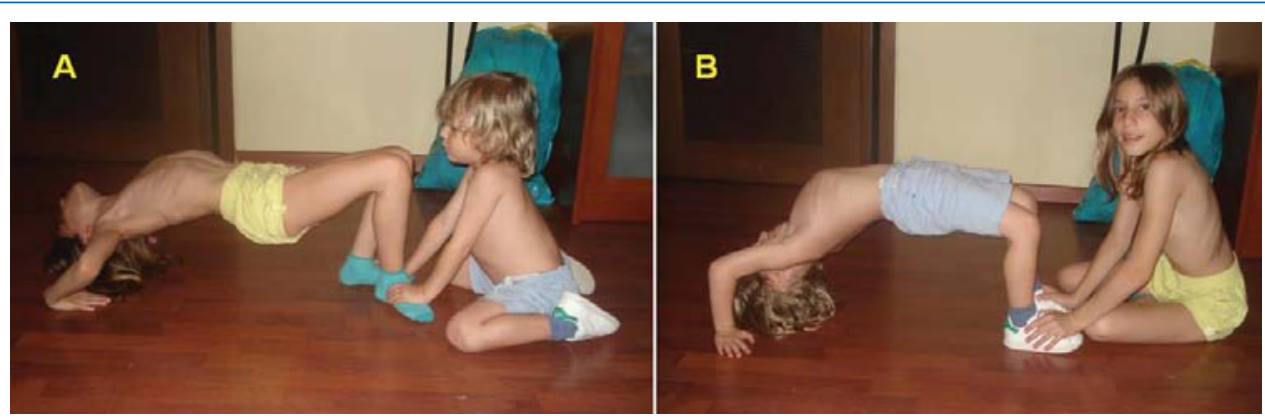

Figure 70: The arching of the back is impaired in the patient Nefeli (A). In the four-year-old brother the arch of the back is physiologic (B). 


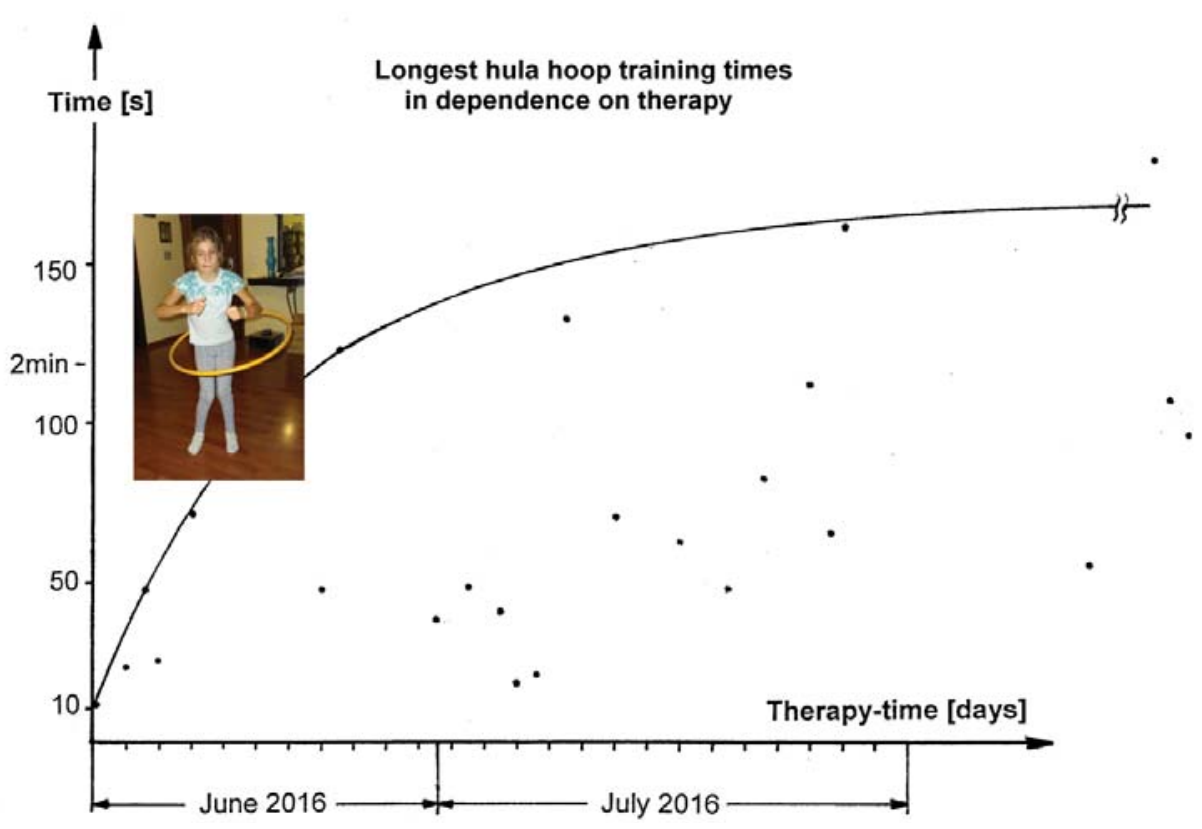

Figure 71: Improvement of longest hula hoop exercising times in dependence on therapy. Note that the individual times varied much.
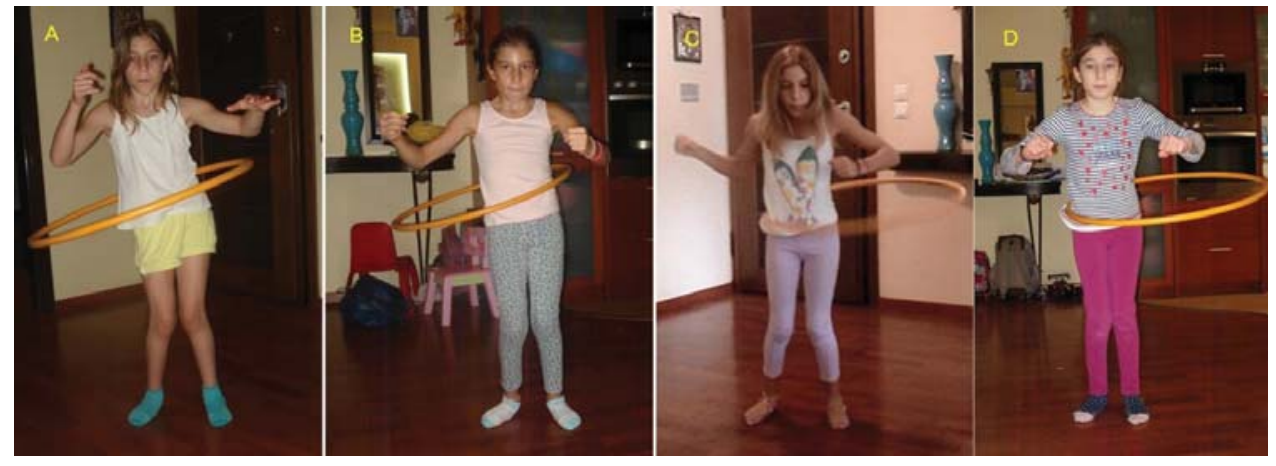

Figure 72: Improvement of the straightness of the trunk during hula hoop. The treatment time is 12 months in A, 18 in C and 22 months in D. The improvement of straightness may not be characteristic for the improvement of her scoliosis.

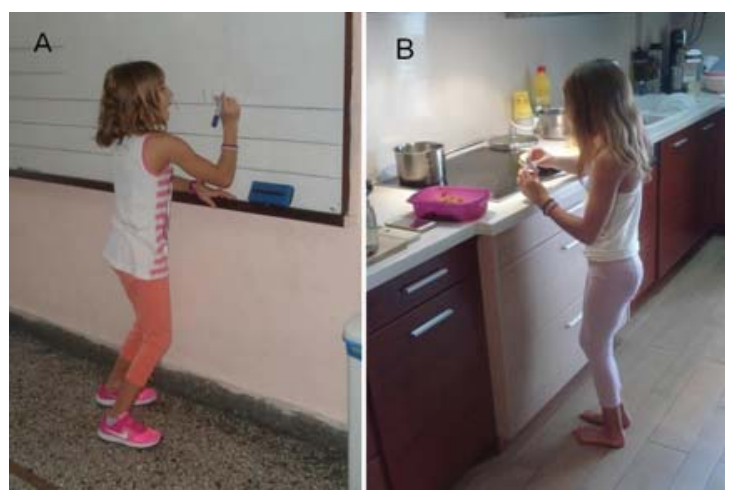

Figure 73: Natural standing of the SCI patient at school following 12 months of therapy (A). After 18 months of CDT she stands straighter during working in the kitchen (B). The patient prefers to be bare foot (B) or to wear socks to have more input from the soles of the feet for better balance because of the damage of the fasciculus gracilis. 
Also, other movements improved. She could crawl better in the more for her difficult pacegait coordination and during walking she could better lift the heel of the 'difficult' right foot. During pace gait crawling, the coordination between arms and legs became better and the feet were positioned more physiologically.

The getting up from the floor after falling also improved with ongoing therapy. After 18 months of therapy Nefeli could get easier up from the floor than after 12 months of therapy, even though she still often lost the balance during getting up.

3.3.19. Improvement of the coordination dynamics: When the patient Nefeli with a SCI Th10 became able to turn by herself on the special CDT device, the coordination dynamics (CD) could be measured. Even though the CD varied very much, they improved with time.

Figure 74 shows the overall improvement of the functioning of Nefeli's CNS quantified by the coordination dynamics (CD) values. The CD values can transiently get worse during repair due to changes of the neural networks. During CDT the nervous system learns quickly to optimize neural network functioning again after changes by improving its phase and frequency coordination among neuron firing.

But CNS functioning, quantified by CD values, can get also worse due to an illness as for example a urinary bladder infection.

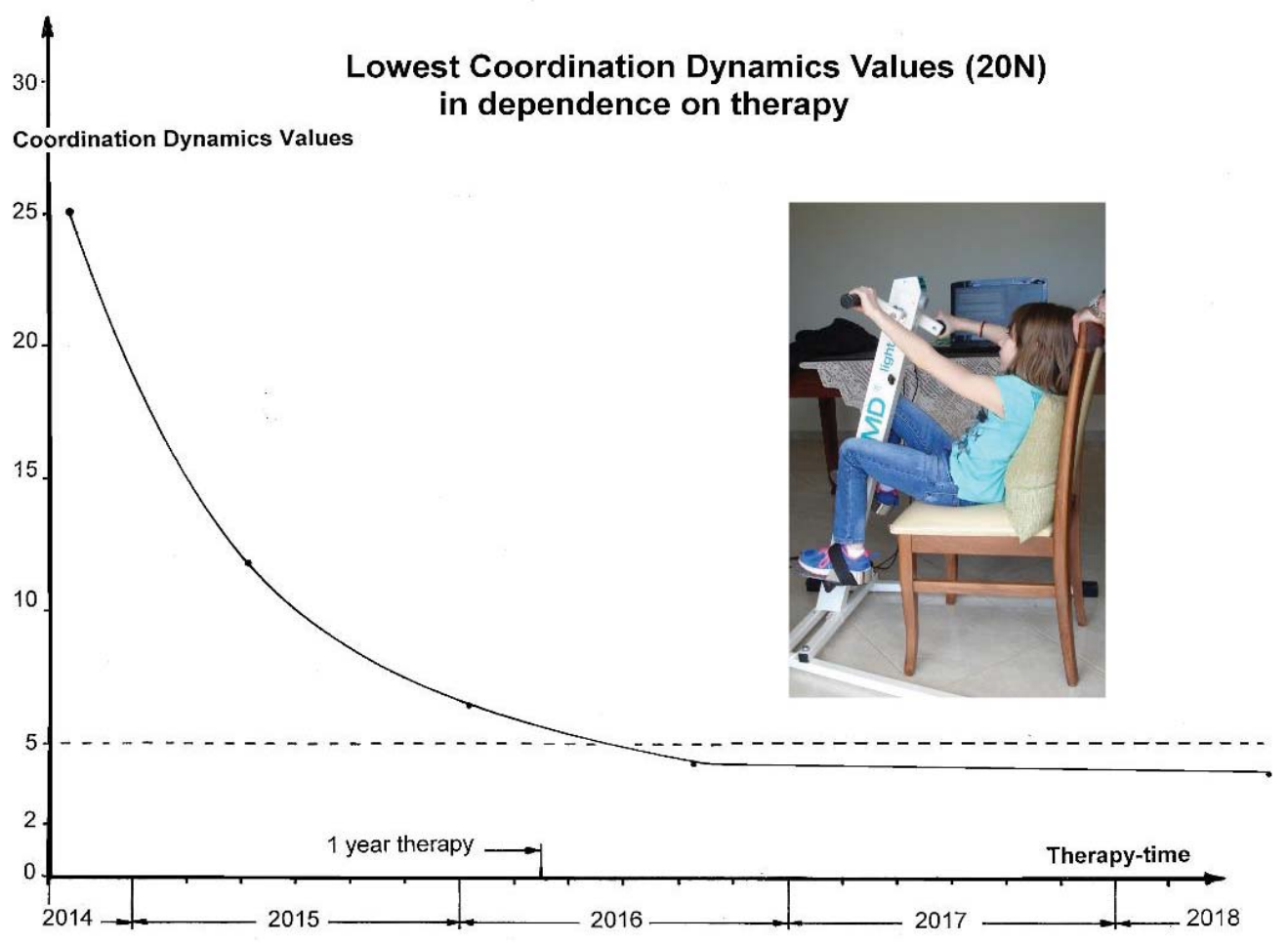

Figure 74: Improvement of the low load coordination dynamics best values with therapy.

3.3.20. Begin of repair of the sympathetic nervous system: The Author noticed the sweating below the injury level because he was supporting her training every day (Figure 77). The start of the re-innervation of the lower body by the sympathetic nervous system division is of importance also for urinary bladder functioning. During the day Nefeli learned to mainly control the bladder via the somatic external sphincter (striated muscle). But at night she was sometimes getting incontinent because the internal bladder sphincter (Figure 27; sited where the $\mathrm{M}$ is; smooth muscle), controlled most likely by the sympathetic nervous 
system, was not fully working again so far. Nefeli could secure the continence at night with the external sphincter. But then her sleep would be only shallow. Deep sleep is necessary to get neural network repair into the long-term memory. She was therefore wearing diapers at night for safety and a deep sleep. Since the sympathetic nervous system started to re-innervate the lower body, it is likely that in a years' time the internal sphincter is functioning better, so that she will also be continent at night. The re-innervation and repair of the vegetative nervous system needs more time, probably because the autonomic efferents are thinner (Figure 9) and the system is more distributed with many plexuses so that the regeneration needs more time.

3.3.21. Psyche and continence: With further repair of the sympathetic nervous system, also the internal bladder sphincter will improve in its functioning, that means also the patient will become continent at night and does not need diapers any more at night. But the continence at night is also affected by the psyche of patients. The stress from the cancer and the SCI may make her also incontinent at night. Healthy children at an age range between 5 and 10 years may also become transiently incontinent at night if they are exposed to strong stress as for example when they lose the parents.

\subsection{Spinal cord injury repair within 2 years}

3.4.1. Fading of regeneration of the spinal cord was probably caused by inefficient treatment and/or beginning of puberty : After a period of two months with nearly no exercising the above reported regeneration of the spinal cord of Nefeli seemed to have waned. The progress with exercising was only minor. To start the regeneration of the spinal cord again by exercising at the limit for approximately 3 months was not possible because her puberty seemed to have started. As she said by herself, she had no power anymore and also no motivation to train at limits. The training was now too boring for her, even though when exercising on the special CDT device she could see films on the monitor of the special CDT device. The movement support by the Author (Figure 77) to increase Nefeli's motivation helped but was not sufficient to start the regeneration of the spinal cord again.

It is known in rehabilitation, that during puberty the patients with CNS injury can get much worse. Therefore, the Author was pushing the treatment as much as possible to keep the level. But how can one motivate the patient and the parents if there is only minimal progress.

3.4.2. Natural therapy: Hippocrates' ancient wisdom that "natural forces within us are the true healers of disease" is in accordance with the strategy of CDT therapy to use natural means (mainly movements) for CNS repair. The question remains, how to find (identify) and stimulate the natural strategies, especially to achieve structural repair. After denervation of one leg of a rat, the rat often eats up the denervated leg, because the leg has no sensitivity and it hinders the animal's movement. If the rat knew that within a few months the leg will be re-innervated [72] and the feeling and the functions will at least partly recover, it would not eat the denervated leg. Humans, on the other hand, have the possibility to find out, understand, and use natural means of regeneration and leave destructive operations and therapies out.

3.4.3. Wheel devices of SCI patients for playing, competition and transportation: A psychological problem in SCI patients is that they are sitting too much in the wheelchair (Figure 75A) and have to look upwards to others instead of being in eye height with partners of a conversation. Psychologically is would be very good if they could stand at least for some time in a conservation to be equal to the opponent.

In sport of SCI patients, one should therefore try to bring the patients into an upright pattern and train it for and during competitions. But what is done in sport for SCI patients, they bring the patients back into the lying position (Figure $75 \mathrm{H}$ ) instead of bringing them into a standing position. Such sport competitions for SCI patients are therefore unethical because it hinders SCI patients to reach the upright position and the equality to healthy persons.

With the improvement of the trunk stability, the patient Nefeli (Figure 75A) became able to ride a bicycle 

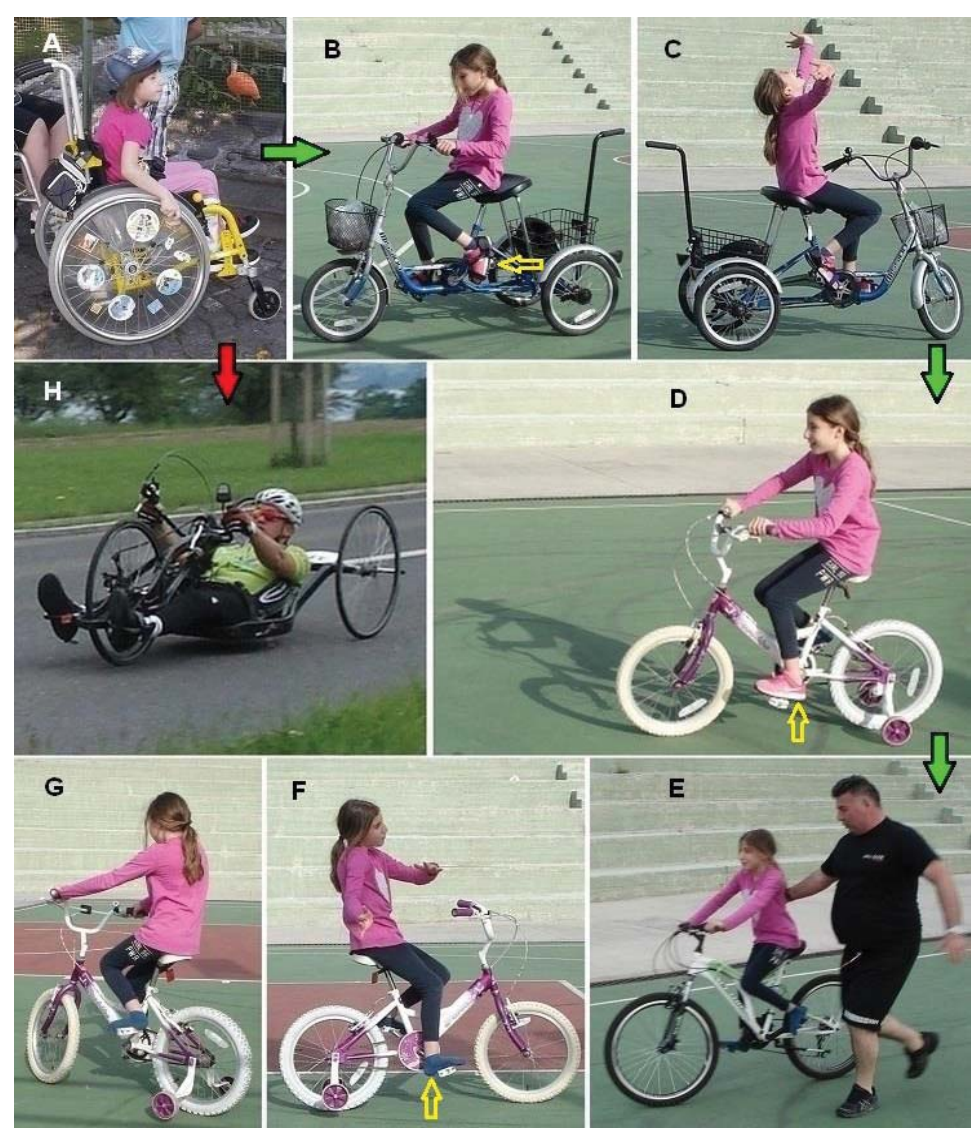

Figure 75: Relearning to ride a bicycle (A-G) instead of riding wheel instruments in the lying position (H) of patients with SCI. In B the feet are fixed. In C Nefeli is demonstrating the improvement of trunk stability. In D through G the feet are not fixed. E. With support of the father, Nefeli can manage a bit to ride a normal bicycle.

with three wheels and fixed feet (Figure 75B). Since also other children used that three-wheel-bicycle (not shown in Figure 75) it was more a play with other healthy children than a balance training. Figure 75C shows that she really liked it to be on the three-wheel bicycle. With further improvement in the short-term memory, Nefeli became able to use a four-wheel bicycle without feet fixation (Figure 75G). Then Nefeli became able to use a normal bicycle with support of the father (Figure 75E). During playing Nefeli trained to improve movement patterns and balance (Figure 75G). Figure 75F emphasizes once more that she really enjoyed the playing/riding with the different bicycles together with healthy children. In Figure 75F the feet were not fixed as in Figure 75C.

A real progress Nefeli achieved with further trunk training, approximately one year later, was, when she learned to ride a normal bicycle at an age of 12.5 , seven years after the SCI, first time in her life (Figure 76). The learning was supported by letting her exercising only with legs on a special CDT device during playing with the iPhone or when hearing music and moving with the music (Figure 83). Such leg training improved a bit the volitional activation of leg movement patterns, necessary for walking, running and cycling.

The practical training for riding a two-wheel-bicycle was shown in Figure 75 when Nefeli exercised posture, balance, leg and feet movements on different kinds of three-wheel-bicycles. Then the step was done to the normal two-wheel-bicycle. Before the first important trial she was nervous and was eating her finger nails. She wanted to ride a normal bicycle to be more normal, but she was afraid because of the $\mathrm{SCI}$. Father and Author pushed her and the riding was going - she was able to ride a two-wheel-bicycle. Of course, she liked it. 
She had no balance problems. But she had to fight with leg spasticity and not sufficient volitional muscle power in the legs. The power of the forefeet was too little to real push the pedals with them. She had to concentrate strongly (Figure 76A) onto her feet not to slip from the pedals. When slipping with the feet from the pedals, she could still keep the balance (Figure 76C). She managed also to ride slalom (Figure 76B). Only with climbing and leaving the bicycle she had spasticity problems. The problem not to slip from the pedals can most likely be solved when using pedal fixations of healthy cycling athletes. In spite of all the problems, she still enjoyed it very much to be closer to the healthy children and move more quickly about. Nefeli had not learned to ride a bicycle bevor the SCI at an age of 5. Being able to ride a normal bicycle and holding the balance has consequences for her later life. Nefeli became not only able to ride a normal bicycle, but became thus also able to ride an e-bike or a suitable small motor bike in the future for being independent without a car. Being able to cover a distance with a bicycle is an independence with a small cost-benefit ratio and will improve her health.

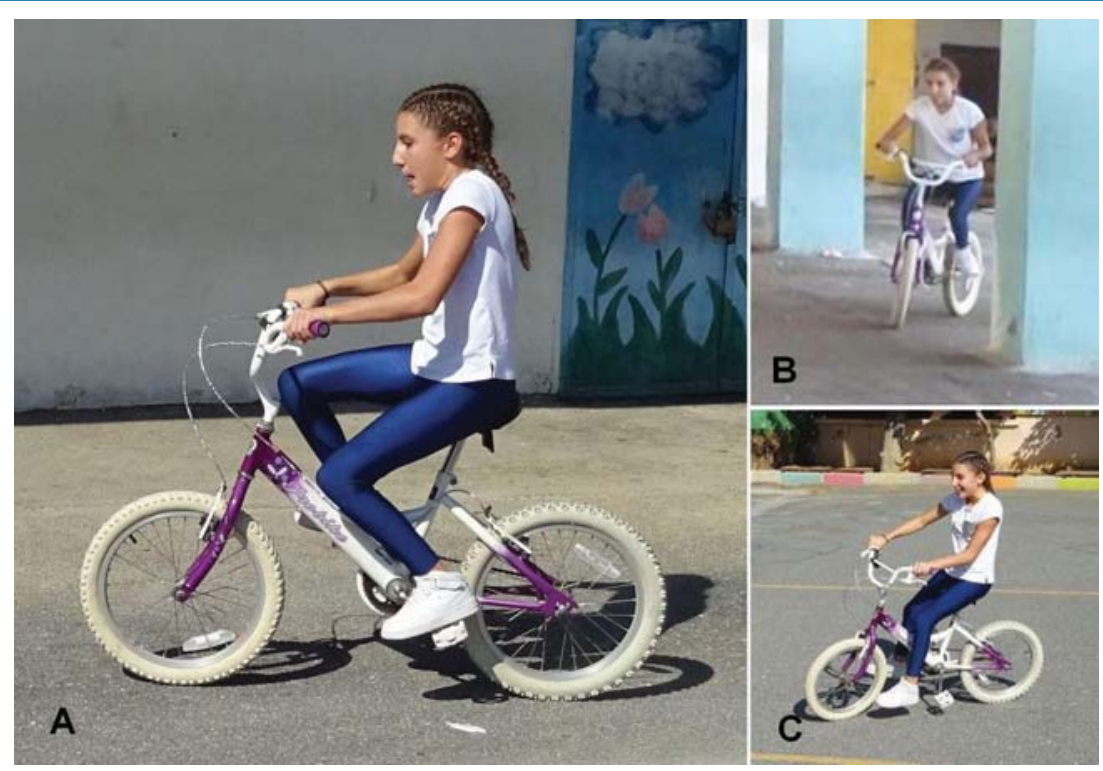

Figure 76: The SCI Nefeli just after learning to ride a normal bicycle at an age of 12.5 years (A). She manages also to ride curves (slalom) (B). She still has problems to keep the feet on the pedals, but when she slips from pedals, she has no problems to keep the balance (C). Note that she has to concentrate very much to keep the feet on the pedals (A), seven years after the SCI injury.

In older patients who could ride a bicycle before the incomplete SCI, the old-learned movement cycling can be used for repair.

\subsection{Spinal cord repair within 2.5 years}

3.5.1. Upgrading of therapy to enhance the efficiency of repair: After the cessation of the regeneration of the spinal cord and no substantial further SCI repair, a new effort was undertaken for a further repair of Nefeli's spinal cord and an improvement of walking, running and balance, bladder and bowel functioning. To achieve a further regeneration of the spinal cord, the therapy was changed and intensified in the way that Nefeli trained at least 55000 turns (up to 75000) per week on the special CDT device and trained walking, running and jumping.

In a neuro-rehabilitation center for children in Slovakia, where they also use special CDT devices, they argue that one session (21 min) per week helps to improve CNS functioning. Children with minor impaired CNS functioning can turn in 21 min 1000 to 1500 times. In comparison to this very low intensity, Nefeli trained now approximately 40 times more intensive. For several months she trained approximately only 13000 times per week on the special CDT device and could not fully hold the level. In relation to this lowlevel intensity, Nefeli trained now 5 times more intensive. Progress in repair can be expected. 


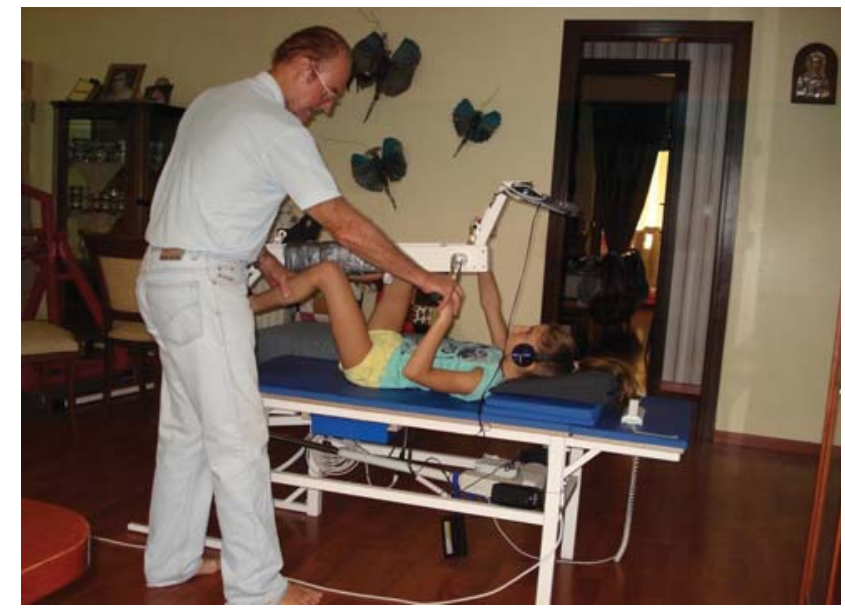

Figure 77: Exercising on the special CDT device with coordinated support by the Author. The Author recognized with the left hand when Nefeli started to sweat in the leg first time after the SCI, which means that the sympathetic nervous system re-innervated the body below the injury level Th10.
To achieve at least 10000 turns per day 5.5 times per week the strong support of a training person was needed. On one hand, such support will reduce the efficiency of repair by movement-based learning because less muscle power (motor action) of the patient is needed. On the other hand, the coordinated touch input to the hand and leg (Figure 77), which means across the SCI levels Th10/11, will increase the efficiency of movement-based learning. That supported exercising on the special CDT device is also substantially improving the functioning of the neural networks of the human CNS is backed by the finding that a coma patient could be brought out of coma and re-learned to speak [67] when exercising with him mainly passively during 6 years.

When touching the skin, specific input patterns enter the CNS as shown in Figure 10

for a simple pin-prick. When supporting the patients turning as in Figure 77, coordinated touch input is repeatedly entering many thousand times per day the CNS and especially the intrinsic apparatus of the spinal cord in rostral and caudal directions. This coordinated touch input 'tells' the intrinsic apparatus of the spinal cord what it has to learn for repair in addition to the input from joint, muscle (muscle spindles), tendon (Golgi tendon organs), fasciae, joint capsules and connective tissue receptors, which is mainly unconscious. The Author supported Nefeli's leg at different places to change the input to the spinal cord and to activate also those tract fibers at the SCI site (Figure 44C) where neurogenesis and dendrite and axon growing are urgently needed for spinal cord regeneration. Especially tract fibers and networks at the border to the injury site, indicated in Figure 44C by a bended arrow, have to be activated. Also stem cells are needed there and have to be activated for migration and proliferation. Even a massage of the triceps surae was sometimes performed in coordination to the hand input to use all possibilities to activate the critical spinal cord network site for neural repair. The patient Nefeli preferred to have support to both hands. But for neural network and tract fiber repair, the input to hands and legs is more important, because the coordinated input is over the injury site Th10. As for the unconscious receptor input, also the coordinated touch input will activate more tract fibers and networks at the injury site (Figure 44C), because nerve cells are coordination receptors (Figure 43) and with more coordinated input the threshold for nerve cell activation is reached earlier and more information can cross the injury site for movement-based learning. For activation changes of the intrinsic apparatus of the spinal cord, different legs, hands and touch places were used for turning support. The seemingly simple training support was also used to specifically activate certain parts of the intrinsic apparatus of the spinal cord.

The supported turning was generally performed at high frequency between 1.5 and $2 \mathrm{~Hz}$ and additionally transiently up to $3 \mathrm{~Hz}$, in similarity to normal high frequency exercising during development. Such exercising was a big load for the patient and the supporting therapist (Author). But as for different sensory modality input, also with more frequent afferent and efferent input to the intrinsic apparatus of the spinal cord, thresholds for nerve cell activation are reached oftener and more communication over the SCI levels Th10/11 is possible for a higher efficiency of movement-based learning.

3.5.2. Further improvement of jumping and running: Figure 78 shows that Nefeli learned to jump better on springboard. In Figure 78A-C she jumped nicely in anti-phase by herself. The jumping in rotation (Figure 78D,E) is still not symmetrical because of the scoliosis. To right (D) she can rotate more than to left (E). The jumping in abduction (F) and adduction (G) became quite good. She learned to coordinate the Giselher Schalow, Switzerland ISBN: 978-81-943057-0-5 

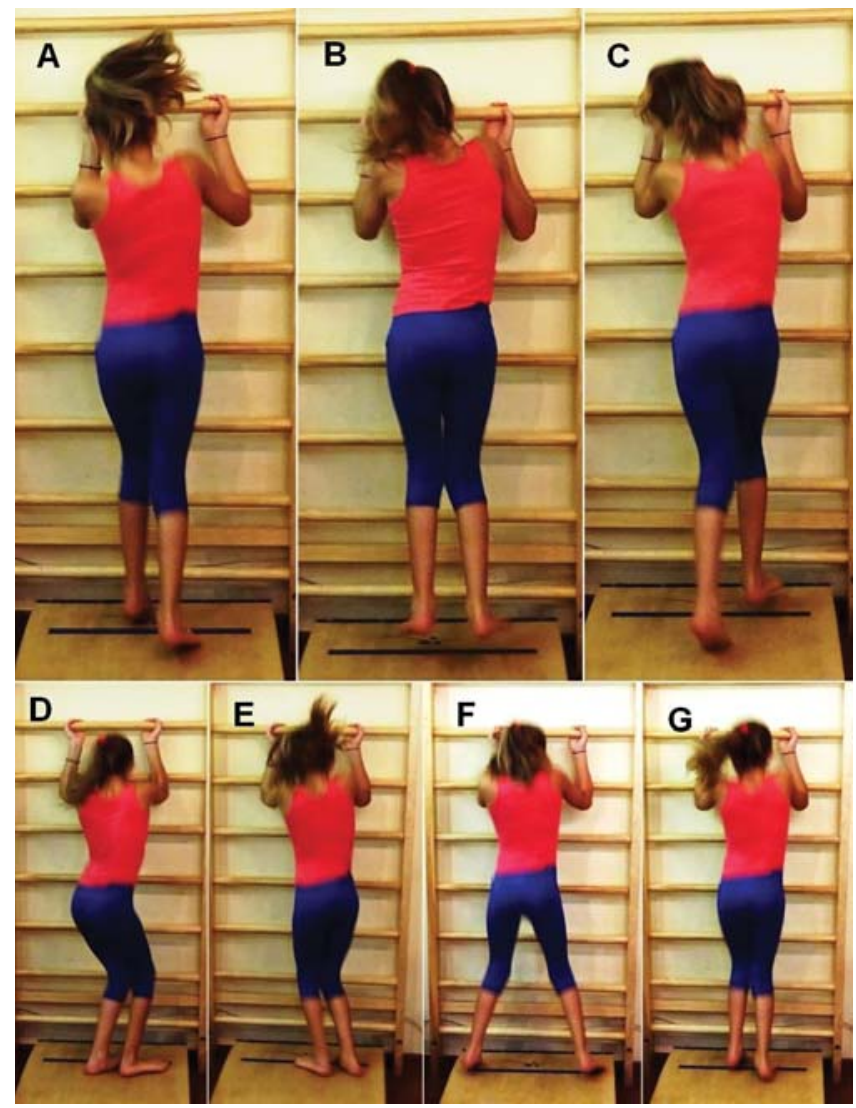

Figure 78: Jumping on springboard of the 11-year-old SCI patient Nefeli after 2.5 years of CDT. Jumping in anti-phase (A-C), in rotation $(D, E)$ and in abduction-adduction $(\mathrm{F}, \mathrm{G})$.

leg movements not to slip over the edge of the springboard (F).

The running also improved. When she became able to lift and flex more the knees, she needed not so much anymore to swing out the legs (Figure 79C) and the running performance improved (Figure 79A). The same holds for walking (Figure 79B).

Being able to jump and run better, Nefeli became able to jump a bit during running, which was big fun to her and was good for the motivation to train further close to the limit. A lot of power for the jumping she was taking from the swinging of her arms.

An impediment of good and fast running was the spasticity of the pelvis (Figures 70A, 80A). Partly it was exercised on the special CDT device with a more stretched pelvis, which was hard work when performed without support and also with support by the Author, because it has to be turned more against the power of spasticity.

A second exercise to reduce the flexor spasticity of the pelvis (Figure 80A) became possible when she learned to run faster. With very fast running of a short distance she could mainly overcome the pelvis spasticity

(Figure 80E). This shows that every little bit of performance improvement has also consequences for other movements.

\subsection{Spinal cord repair within 3 years}

3.6.1. Problems of spinal cord injury repair during puberty: After three years of CDT of varying intensity, the overall motor functions were not much better than after 2.5 years because Nefeli had trained

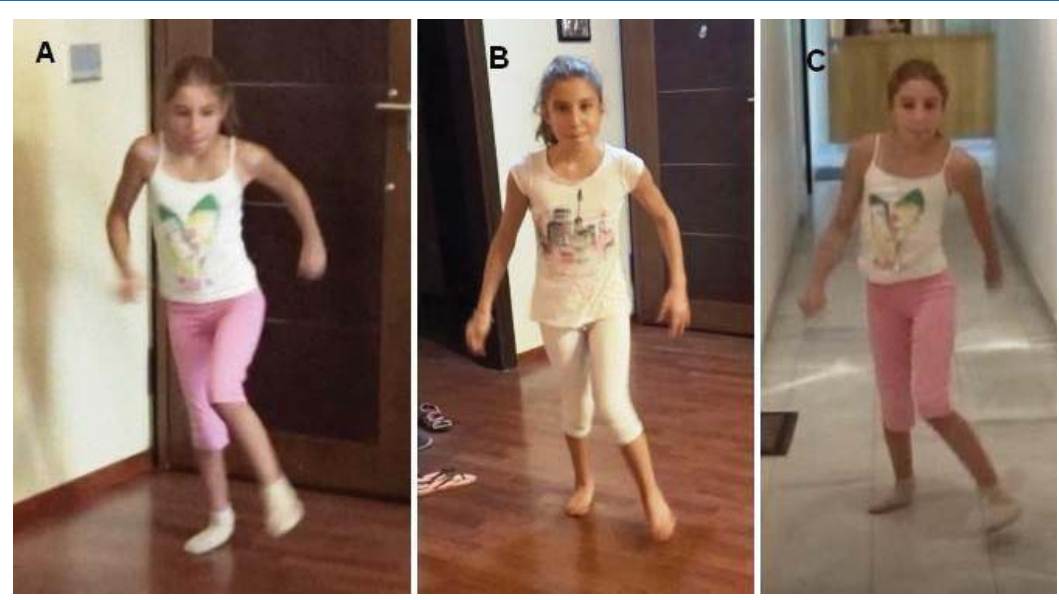

Figure 79: Improved running performance (A) of the SCI patient because of being able to flex and lift more the knees instead of swinging the leg outwards during walking (B) and running (C). 

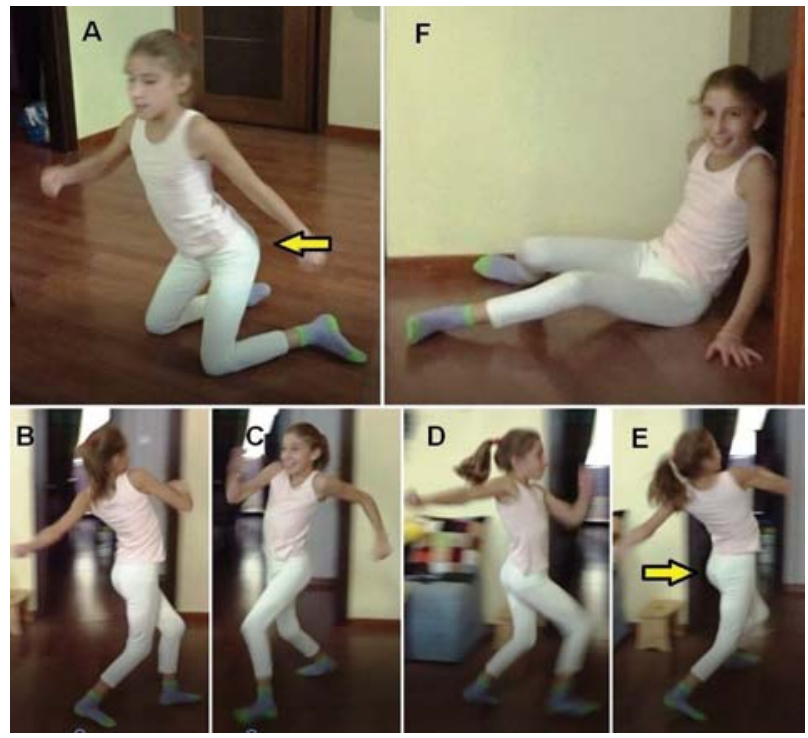
SCI at the level of Th10/11. A. During walking on knees, the pelvis is too much backwards mainly because of spasticity. B-E. During running the patient is able to bring the pelvis forward, especially in E. F. After running 10 times the distance of $6 \mathrm{~m}$ the patient was exhausted but happy. Note that the patient is strongly using her arms to increase the running speed. A high-speed camera would have been needed to improve the quality of the pictures.
Figure 80: Running to improve pelvis positioning in a patient with a

too little in the meantime when the Author was absent and the parents did not take the therapy over. When the Author trained with her, she was getting better and when he was away, she did not get better or even got worse. Proper treatment of a handicapped infant or child consists of close cooperation between an active parent and an active therapist. The puberty seemed to have worsen the situation. It is known that during puberty the brain functions are getting worse at least in children with cerebral palsy.

In the age range between 11 and 14 years, the coordination dynamics values of healthy girls and boys got transiently worse (Figure 82 of [19]), indicating transient impaired brain functioning, probably induced by the changes of hormones. In this period patients with brain or SCI may get worse. The main therapy task in that age range is therefore to keep the level of repair and if possible, to increase it. The problem of mental discipline is occurring.

Still some motor functions improved. She could do better with the hula hoop. This movement is of importance because the trunk muscles are trained and also the neural networks at the injury site (Figure 44C) are activated to hopefully induce some regeneration of the spinal cord. Nefeli could manage the hula hoop for a long time with one ring and could even play a bit with the balloon with another person at the same time (Figure 81A). With two rings she could do hula hoop for approximately 10 to 20 seconds (Figure 81B) and with three rings up to 5 seconds (Figure 81C). Then she became able to do hula hoop and swing additionally rings with the hands (Figure 81E,F,G). Many school maids could not do the hula hoop because of overweight. Rotating the body with several rings probably activated more trunk muscles around and below the spinal cord segments Th10/11.

It is very unlikely that Nefeli did not get much better in her motor functions because the CDT had reached with her its limits.
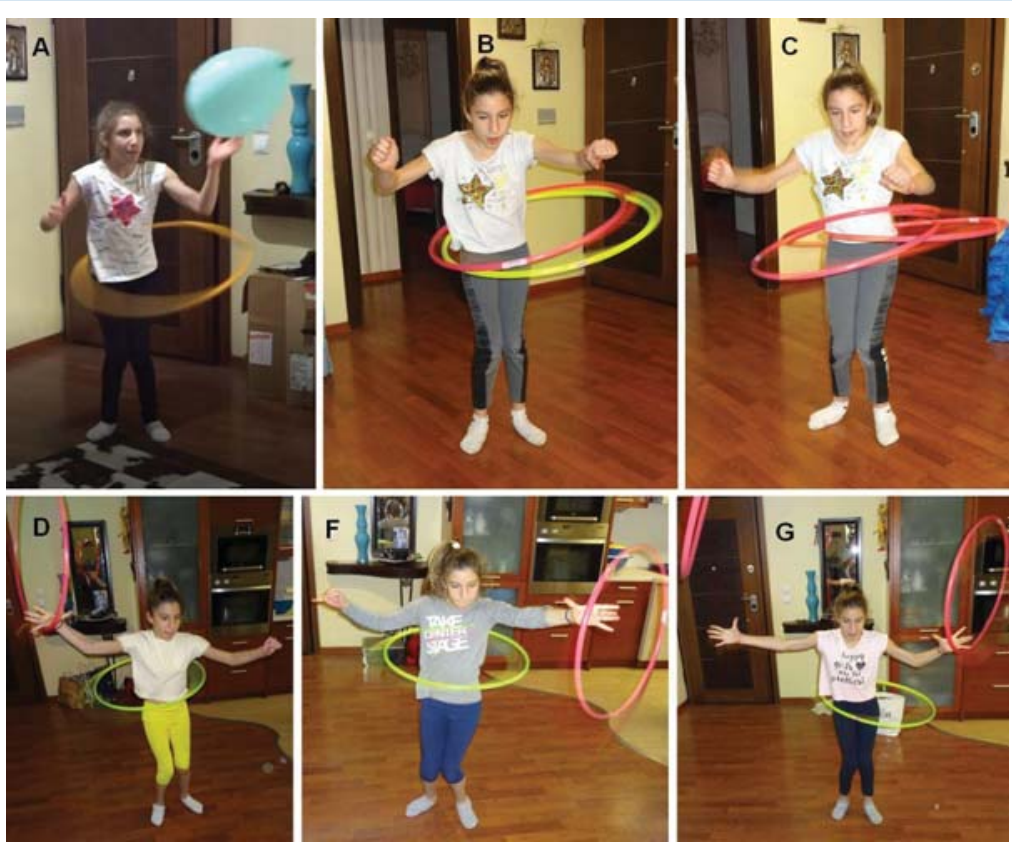

Figure 81: The SCI patient Nefeli during performing hula hoop and playing at the same time with the sister/Author with a balloon. B. Nefeli during hula hoop with two rings. C. With three rings, which was possible only for a few seconds. D,F,G. Nefeli during hula hoop and swinging additionally rings with the hands. 
The coma patient Manolis, got continuously better even after 6 years of CDT and being 4 years in the coma [67]. But CDT was administered to him at the limit, that means with 20 hours per week continuously.

3.6.2. Improvement of urinary bladder functioning: The vegetative functions of Nefeli improved further. After three years of CDT she had no bowel pain anymore, the urinary bladder infections did not occur anymore and the storage volume of the bladder had increased. When CDT was started, she had a urinary bladder storage volume of $50 \mathrm{ml}$, then $70 \mathrm{ml}$ and after three years of CDT the storage volume had reached $100 \mathrm{ml}$. Even once a storage volume of $140 \mathrm{ml}$ was measured. Her normal healthy urinary bladder storage volume would be around $200 \mathrm{ml}$. It seems therefore that her sacral and pontine micturition centers were still not fully repaired. Nefeli could mostly keep the fluid from the first desire to void to the second desire, which increased the storage volume. Still the detrusor seemed not to have the full power to empty the bladder, because once Nefeli could empty the bladder with $70 \mathrm{ml}$ (only partly) and two minutes later again with $70 \mathrm{ml}$ (fully).

It was shown in Figure 30A how the detrusor is activated in the rather healthy case and in Figure 30B following SCI. Nefeli's bladder functioning was probably somehow between the cases 'A' and 'B'. The detrusor activation was probably still a bit undulating (working unregularly), that means the detrusor was not getting the full-strength activation from the micturition centers and could not fully relax between the voiding's. More CDT is needed for a further repair of the micturition centers.

3.6.3. Treatment of 'iPhone' mania and the coordination of fins of fish (von Holst): The Author encountered substantial difficulties in motivating Nefeli to train close at limits. Many parents with cerebral palsy children have similar problems. The Author lost a patient for training partly because of her addiction to the iPhone. When Nefeli got a new Apple iPhone, she preferred to play more with the iPhone than exercising with the Author. The Author used two strategies to reduce this mania.

First, Nefeli was allowed more to play with her guinea pigs. But she had to care about them, which means cleaning, building simple houses for them and other things.

Second, Nefeli was motivated by the Author to work more with her fishes. She anyway liked her fishes and cared about them. But now he showed her, that the fishes move their fins in coordination (Figure 82). With the Author, Nefeli took a magnifying glass and looked for the coordinated moving of the fins, even though these small fishes were not suitable for this children's research. The intention was not to start with her biology studies as von Holst did long ago, but it was the idea to make the working with the fishes more interesting and attractive.

Actually, the biologist von Holst started the research of coordinated movements and oscillators, when studying the coordinated moving of fins of fish [73]. The Author made then the step from biology to medicine that means the step from animal research to human research, analyzed movement patterns at the single-neuron level and developed treatment for patients. Von Holst's result of the interaction and struggle between 'Beharrungstendenz' and 'Magneteffect' create an infinite number of variable couplings, and in essence form a state of relative coordination [73] which is quantified by the Author by the organization principle 'phase and frequency coordination' in the human CNS (Figure 12). Von Holst's 'Magneteffect' is

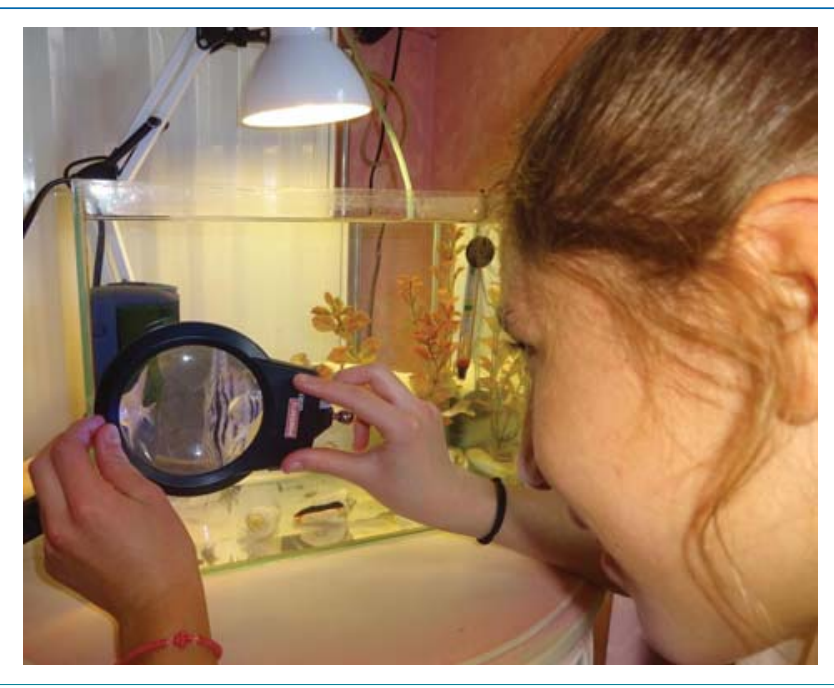

Figure 82: Nefeli is watching the coordinated moving of the fins of fishes. 
quantified by the coordinated firing of oscillators and the 'Reafference Principle', which largely deals with the interactive processes between the CNS and the periphery by the coordinated firing of secondary muscle spindles with other CNS neurons ( $\alpha$ and $\gamma$-motoneurons). In the framework of coordination dynamics, the coordination tendency, named 'Magneteffect' by von Holst, can be understood as the remnant of the attractor corresponding to absolute coordination [21].

The idea that the nervous system supplies coordination dynamics [21] rather than particular coordination patterns [74] could be viewed as an interpretation of von Holst's early work on relative coordination. Orthopedic surgeons believe more or less that the CNS supplies particular muscle function. On the basis of a gait analysis, they decide what muscles and tendons have to modulated to improve walking. This symptomatic therapy is out-of-date because there exists a causal therapy, namely CDT.

Even though Nefeli liked it very much to work with the guinea pigs and the fishes, it was still difficult to compete with the iPhone playing.

\subsection{Spinal cord repair within 3.5 years of therapy (September 2018)}

Following 3.5 years of CDT of changing intensity and being 12.5 years old, Nefeli did not get much better. Still the Author thinks that the limit of CDT has not been reached in her. The therapy has to be administered to Nefeli more continuously, which means that in this case the parents have to get more involved in the therapy, even though the load on a family with a disabled child is large. Hopefully the puberty changes are getting over, so that Nefeli has more motivation to train again. The efficiency of CDT has to be enhanced and, last not least, Nefeli needs some progress/success for motivation. Even for the therapist (the Author) it is hard to work with a patient when the progress is only marginal.

The only real progress Nefeli achieved in the last 6 months was that she learned to ride a normal bicycle at an age of 12.5 , seven years after the SCI, first time in her life (Figure 76). The learning was partly prepared by let her exercising only with legs on a special CDT device during playing with the iPhone or when hearing music and moving with the music (Figure 83). Such leg training improves a bit the volitional activation of

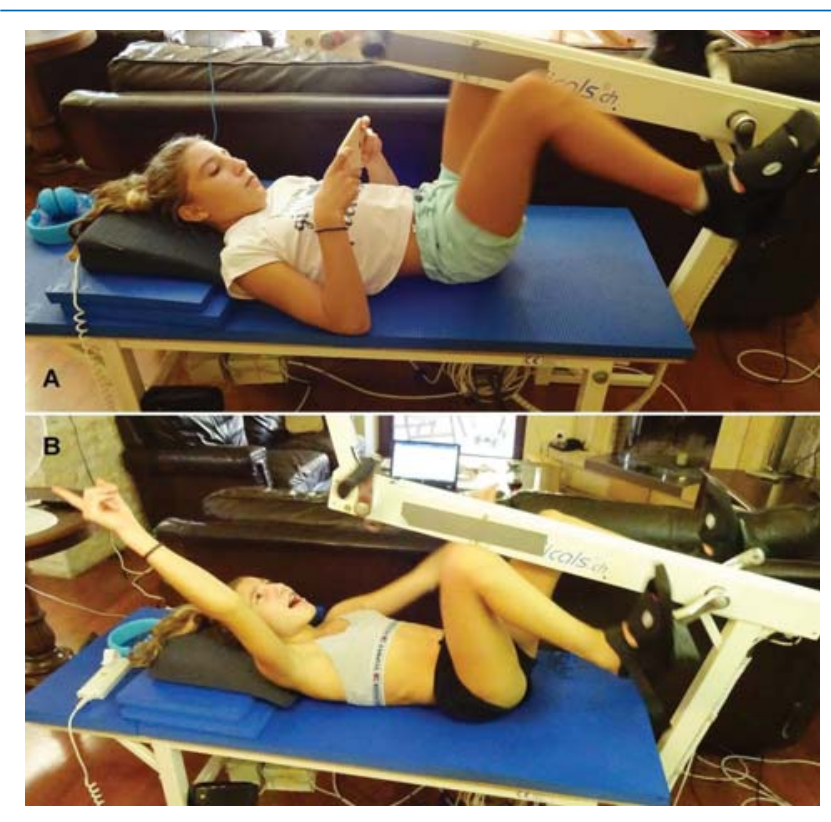

Figure 83: The SCI patient Nefeli during exercising only with the legs while playing/working with the iPhone (A) or singing and moving (B). While playing a bit crazy during CDT (B) is beneficial for repair because it keeps the patient in a good mood in spite of the SCI. Being addicted to the iPhone (A) is mostly not beneficial for repair. leg movement patterns, necessary for walking, running and cycling. Mostly Nefeli was exercising with little clothes because of high room temperature and not fully repaired temperature regulation (sweating) below the injury level Th10/11. Also, for judging her spasticity during treatment or posture, it was beneficial to see the uncovered legs as in orthopedic.

The transient lessen of the regeneration/ repair in Nefeli emphasizes the importance of continuous treatment on the long-term, especially in children during development. It would be interesting to see how animal researchers could simulate such repair over several years in rat or mice. In the patient Nefeli, the administered treatment lasted so far 3.5 years, in the former coma patient Manolis 6 years and in brain injury patient Benjamin over 18 years. It needs to be known what repair can be achieved in what time period with an efficient therapy applied aggressively and continuously. 


\section{Discussion}

\subsection{Limitations of basic science research}

There are many inadequacies in the current research in SCI. Animal research is mainly concerned with structural repair and the regeneration of the spinal cord in animals. The aspect of functional recovery is rarely understood and never analyzed. Even though structural repair is important in $\mathrm{SCI}$, the researchers rarely discuss the difference in the potential of regeneration between animals and humans. In the goldfish, for example, the spinal cord regenerates spontaneously. But what inferences can be derived from this regeneration that can be relevant and applicable to humans? Presented gross human anatomy may make a casual reader believe that the animal data are indeed applicable to humans. However, the functional recovery is not measured thoroughly. Morphological data at the cellular level, like the degree and extent of growth and regeneration of axons across the injury site, is often not correlated with the functional recovery. The functioning of newly grown axon has to be proven in the context of neuronal network functions and not only by the improved movements of the treated animals, because in animals there exists also spontaneous recovery and functional reorganization, especially if only $50 \%$ of the cord was destroyed. Actually, fiber counts in different ascending and descending tracts would be needed to assess what percentage of tract fibers need to be regenerated for meaningful recovery in humans.

\subsection{A need for scientific and clinical human approach}

The diagnostics at the beginning, during, and at the end of therapy is of particular importance to differentiate between spontaneous recovery and the improvement achieved by the treatment. Since spontaneous recovery may occur for up to 1 year after the injury, long-term studies are needed. In the case of a partial SCI, most of the recovery occurs between 2 to 6 months after injury and to a lesser degree up to 1 year. In the present patient Nefeli, the treatment was started 4 years after the injury and lasted for more than one year; thus, the recovery cannot be attributed to spontaneous recovery.

In the case of complete SCI (as assessed by MRI), only very limited spontaneous recovery occurs and the potential for functional reorganization is very low, since the majority of the distal cord is disconnected from the supraspinal control. In such an injury even the combination treatment of stem cell therapy and CDT did not bring about significant improvement. However, most of the spinal cord injuries are incomplete. Even in the case of a severe injury, a significant part can be salvaged by immediate removal of compression of the spinal cord by surgery (Figure 5), what was not done in Nefeli and the stabilization of the spine to minimize progressive mechanical injury.

There are many unscientific reports, claiming miraculous recovery in SCI patients, which could very well be attributed to a spontaneous recovery. Most of the rehabilitation centers focus mainly on care and general maintenance of patients and administer inefficient and arbitrary treatment that does not utilize the full potential of recovery in patients with partial SCI. Patients want more choices than the 'no hope' approach of conventional rehabilitation centers and the 'false hope', given by some physicians and researchers working in the field of animal research, and cell and molecular biology.

Over the last 30 years no significant conceptual or real methodical progress has occurred in the field of neuro-rehabilitation. The current science of neuro-rehabilitation is mostly outdated, as no real review of 
human neurophysiology has been done for many decades. The lack of any perceived need for development and in turn the absence of any efforts in that direction has perpetuated the inefficient, outdated and ineffective treatment modalities in the treatment delivery system. This is particularly relevant considering the fact that the young patients who suffered a SCI have 50 years of their remaining life span that they have to live with a very poor quality of life, while only getting care from the current rehabilitation centers. On the other hand, with optimal and intensive CDT for a few years after the injury, their quality of life can be enhanced substantially in order to achieve independence in daily activities, and most importantly physiological bladder control and may be improvement of the sexual function.

\subsection{Animal experiments are removed from human reality}

Recently it was reported on the spontaneous recovery of a SCI in rhesus monkeys and the important role for primate models in translational disease research [75]. A few comparisons will show that animal experiments are far away from human reality and normally cannot contribute to translational medicine or human disease research.

1. In human patients, as can be seen from Figure 19D, the injury is often in the center of the spinal cord, and not just a half of the spinal cord is damaged as in the monkey experiment (Supplementary Figure 2 of [75]). Neurons in the spared grey matter of monkeys may function as relay neurons. The blood supply is probably less impaired in the monkey.

2. Since in $50 \%$ SCI the spinal cord can mainly be repaired upon CDT [8] and the patient can be brought back to normal everyday life (Figure 19D), there is not much sense of after-developments in monkeys, unless the experiments are designed to be close to the clinical human setting to obtain parameters which cannot be measured in humans. Is it actually justified to kill monkeys for an afterdevelopment? Not to cite existing qualified clinical literature for the repair of a 50\% SCI is also not convincing, especially when claiming that their research is a contribution to translational medicine.

3. The biggest problems in human SCI are the urinary bladder functions [9] and the occurring pressure ulcers due to insufficient blood supply. Before World War II a majority of SCI patient mortality was due to continuing urinary bladder infections. By cutting only one half of the spinal cord in animals one is not getting urinary bladder and skin nutrition problems. The challenge of the future is to cure the $95 \%$ or complete SCI. - The neurosurgeon L.W. Freeman (USA) stated in the 1960's after performing very many SCI repair experiments on dogs, "it is difficult to find persons who do research in paraplegia and to get money for such research; probably World War II is already too far away". If one cuts the whole spinal cord in animals, then one would have to manually empty the bladder by pressing the urine out. Such work seems to be unpopular among researchers. In human patients the bladder is for example emptied approximately every 5 hours by intermitted catheterization (and other ways), introduced by Guttmann from Breslau [1] in the Second World War.

4. The improvement of hand function is also very important in cervical SCI; I have not seen animal experiments tackling the hand and finger problem. A 'tetra' (tetraplegic patient) cannot empty his urinary bladder by intermittent catheterization without sufficient hand and finger functions. Hand functions can be improved in C5/6 SCI upon building of new motoneurons in the human spinal cord, but it needs several years of optimal CDT [32].

5. The repair of the fundamental functions such as breathing, swallowing, and speech in high cervical SCI has also not been tackled by animal research. Obviously, animal research is far removed from the human patient's real problems.

6. Humans need years for development and repair and not only a few months. Animal experimentalists are therefore working on the wrong time scale with respect to development and repair to translate their data to human reality. Again, it seems not to be popular among researchers to do experiments which last over several years. 
7. Translational medicine makes only sense if the researchers have knowledge in animal and human research. A translator can only translate from German to English if he has knowledge in both languages. Also, for peer reviewed journals in translational medicine it would be important to have reviewers which are competent in animal and human research. The competence has to be documented by publications in international journals in both fields.

8. When I was for postdoc education with Sir Bernard Katz at University College London, I learned that there should be no more than 4 authors on genuine research publications. 14 authors of a publication are typical for clinical research where clinicians do not have enough time for research and putting their names as much as possible on the publications of friends and assistances to increase the number of their own publications. By avoiding real research, professors in the clinical field may have insufficient understanding of qualified research and are less open to new developments in medicine.

9. In mouse experiments, half of the spinal cord is cut and treatment success is demonstrated by the staining of neurons and nerve fibers and demonstrating that the mouse can run for example on a horizontal ladder [76]. Whether the regenerated fibers were really functioning was not measured. Something like 50 years ago, there was a treatment for thoracic SCI with which patients learned to move the legs by moving the arms. The information from the upper (arms) to the lower part of the body (legs) took place most likely by skin traction. In a frog, I could not see from the morphology whether axons and synapses were functioning; when trying to understand regeneration, I used morphology and electrophysiology. Sir Bernard Katz, the specialist for the neuromuscular endplate, explained that sometimes denervated motor endplates survive very long and still look quite normal (as if they would really still function). In mouse treatment, success was measured by morphology and making the mouse move on a horizontal irregular ladder and arguing then that the progress is going from animal experiments to human treatment [76,77]. In the human patient with the 50\% cervical SCI, treatment progress was demonstrated by letting the patient run and jump, measuring the improvement of running and jumping, measuring the coordination pattern dynamics (CD) values for different loads (Figure 21) and measuring motor programs and single motor unit firing (neuron level) with surface electromyography (sEMG) (Figures 12,13). And the scientific basis for the repair is the understanding of CNS functioning and repair at the neuron level in human [17-19]. For the time being the progress in SCI is coming from human research and not from animal research. Human data need to be translated to animal data. Universities and research institutions world-wide seem to have an anxiety to touch human neurophysiology and qualified clinical research.

10. In severe incomplete SCI there is the problem of muscle power generation. If not sufficient motoneurons can be activated following SCI, the body tries to compensate for by axonal sprouting to innervate the (denervated) muscle fibers of not functioning motoneurons. With such collaterals the motor units increase in size. In human the motor unit can be increased by approximately $50 \%$. One motoneuron can, for example, supply 6000 muscle fibers when it normally innervates 4000 muscle fibers. In rat the motor unit can be increased up to $400 \%$. Therefore, frankly speaking, a rat can walk already with a few functioning motoneurons, with poor coordination. Further, the power of regeneration is much higher in animals.

11. The problem of smart-phone mania, which disturbs treatment, one is not having in rat experiments.

\subsection{Consequences of false hope}

In Chapter I of [18] it was shown, based on frog data that exogenous stem cell therapy in its present application is not working. The main problem seems to be that the newborn cells cannot be integrated in the existing neuronal networks for various reasons. Cell communication needs to be better understood. During competition a motocross athlete suffered a clinically complete SCI at the thoracic 11/12 levels. Six weeks after the accident the subject began intensive CDT at an up-to-date therapy center. After 6 months of therapy, when further improvements were only marginal, the patient opted for haematopoietic stem cell 
therapy. During two years of stem cell therapy, including 4 sessions of stem cell application, and on-going CDT, improvement remained marginal - no more than what would have been achieved with continuing only CDT. This haematopoietic stem cell therapy did not have any beneficial effect on the repair of the spinal cord in this patient. When it turned out that the costly stem cell therapy had no beneficial effect on the regeneration of his spinal cord, the patient lost the belief in SCI treatments and stopped also CDT. He only continued with some training, according to his opinion, to reduce spasticity. Another patient with a probably complete thoracic SCI was sent by a physiotherapist to the Author to get advice concerning a stem cell therapy in addition to the administered movement therapy. The patient did not consult the Author, because he wanted to have the stem cell therapy administered, whatever the arguments of the Author would have been. He even raised the argument that the Author may be against progress in SCI. Up until two years after the start of the stem cell therapy there was no real progress in repair. The patient stopped the therapy in the physiotherapy place and was doing then his own treatment at home. It seems that he had lost the belief in any treatment. During the treatment at the physiotherapy place no MRI was performed in this patient (messy treatment and diagnostic situation (Switzerland)). It was argued that this is not possible because of the metal fixation. But with a Titan fixation, which is nowadays mostly used, an MRI is often giving quite a good anatomical information about the severance of the injury of the spinal cord. This can be seen if one compares the MRI's of a patient with Titan fixation (Figure 19C) and after its removal (Figure 19B).

Instead of raising false hope that the animal data can be easily used to cure diseases of the human CNS, the universities and research institutions should first do their duty, namely organizing qualified human neurophysiologic research, especially treatment research, and also support such research.

\subsection{Ethics of SCI research, treatment, and clinical trials - false hope from animal treatment research}

'Transplantation of both embryonic stem cells and embryonic stem cell-derived neural (neural or glial) progenitors is able to efficiently promote CNS regeneration in preclinical models of stroke, myelin deficiency, acute SCI [78,80] and Parkinson's disease [79]'. This sentence of an article makes the reader believe that the research made already the step from the animal research to the human research and treatment is already administered to humans. Looking up the references one finds the title: 'Human embryonic stem cell-derived oligodendrocyte progenitor cell transplants remyelinate and restore locomotion after spinal cord injury'. Patients with SCI or journalists think immediately that the SCI problem has been already solved! I have learned that it has to be written in the title on what species the research has been performed. In this case it was the rat. Animal researchers often deliberately choose the words in a way to make the reader believe that this research has already solved the SCI problem in humans. A well-known journal in medicine is even redefining medicine. It makes the reader believe that measurements in mouse are a part of medicine [81]. I have learned in my medical study that medicine has something to do with the cure of diseases in human. Specialist journals and general audience media need to set reasonable expectations of the safety and efficacy of potential therapies to avoid raising and then dashing the hopes of those living with SCI or those in government, those carrying out research, or the general public [81]. High ethical standards are required by researchers, clinicians and journalists to ensure that results are communicated to the general public in a manner that honestly reflects the safety and efficacy of a potential therapy [81].

Medicine is very successful if natural existing repair mechanisms are supported like in surgery or immunology. But if repair mechanisms do not exist, then medicine is not very successful. The repair of the nervous system is very limited and depends on the site of the injury. An exogenous stem cell therapy must be very sophisticated to be better than nature with respect to repair. The integration of new-born cells into the existing neuronal networks is by far not solved. Further, the scientific basis for doing neurobiology is out-of-date. The understanding of the functioning of the CNS in animals and humans has to be upgraded. In physics, for example, the basis is continuously upgraded. In Chapter I of [18] it was shown how complicated the innervation of two kinds of muscle cells by two kinds of motoneurons is (4-cell communication). The innervation and innervation changes during development and repair are probably much more complicated 
in the human CNS. It is too simple thinking to believe that an addition of cells can repair the CNS what the CNS cannot do by itself. CDT, on the other hand, rests on learning which is natural for humans. Academically accepted articles are generating false hope by allowing authors to state that stem cells can potentially be used in different CNS diseases including SCI. The authors are stating it to get funding for their animal research, by making believe that their research has direct consequences for the treatment of human patients. No wonder that practitioners also want to participate in getting money. Instead of warning that some clinics in Asia may look more for profit than for qualified stem cell therapy, the editorials should first think over their own research policies which are giving rise to such unqualified medical treatment. It is not enough to state "The medical promise of stem cells remains real, but largely unrealized for now. The excitement must not be left to dissolve into a muddle of disappointment, frustration and fear because of the practices of a few irresponsible profiteers" [4]. There are principle problems to be solved for stem cell therapy in humans. A few years ago, there was a big propaganda in the TV and other mass media in Switzerland about the suppression of grow-inhibiting properties for the regeneration [76,77] of the spinal cord. It was stated that SCI could be cured in a year's time. It seems to be popular among neurobiologists to raise false hope in society concerning treatment in human patients, as if they have not understood much about treatment of human patients.

If we want to repair function, we also have to measure function in a qualified way and one powerful tool is the electrophysiology, because the nervous system is mainly functioning by electric currents and potentials. It is difficult to understand why electrophysiology has mainly been destroyed world-wide, even though this tool was very successful in neurophysiology in the past. One reason could be that although the younger generations are capable of handling computers or electronic equipment very good, they do not have the manual skill any more to perform complicated electrophysiology. Statistics are needed concerning treatment success beyond the placebo effect, including the occurrences of cancer and CNS instabilities (seizure disorders). But more is needed in medical research than just statistics. Hopefully the globalization does not give rise to the coordination in thinking (in German: 'Gleichschaltung der Denkweise').

It is depressing for a qualified therapist that 'there is an abundance of patients desperate for miracle cures, and one stem cell treatment can bring in tens of thousands of US dollars' [4]. Western scientists and clinicians would argue that controls are necessary to identify unambiguously whether a therapy is safe and effective, some clinicians have claimed that withholding a potential therapy from a patient with SCI is in itself unethical [5]. Many SCI units know about CDT. But the patients are not informed that such a therapy exists! A real change from care to cure seems not to be in the interests of the neuro-rehabilitation centers. More money can be earned with care.

There is too much false hope generated by animal physiologists, neurobiologists, and researchers working in the field of genetics. Without detailed knowledge of the human physiology and pathophysiology, the animal knowledge is only of limited help to cure diseases in humans. False hope also stops the patient from fighting for improvements which are needed for everyday life. Why should a patient fight for three years or more with a movement-based learning therapy to get urinary bladder and some motor functions repaired, if in a few years' time walking can be returned with miracle cells or pills? Such false hope is coming from qualified researchers, when they make believe that the animal data can be easily used to repair the human CNS. Qualified human research is needed and has to be organized. Only if there is an overlap between animal and human research, there is the possibility of rather safe transfer of knowledge from animals to humans. As long as human research is not organized properly, the patients have to suffer or even to die (for example Christopher Reeve). Even monkey experiments are far away from human reality. A monkey cannot speak, write or read, and will never be able to solve 'differential equations' (mathematics). The power of the human CNS is its learning capacity, which is outstanding among different species. A fly demonstrates with how little brain matter sophisticated fly tasks are possible. Qualified human CNS research, including at the single-neuron level, is needed for understanding the functioning and repair of its neuronal networks. 
Authors of recent review articles presented approximately 1000 citations on the repair of the human spinal cord following injury [5,6,79,82]. Less than $10 \%$ of the citations were from human research. Interestingly, the author was not cited, even though his work is widely available online. I learned a lot from these brilliant research articles with respect to animal research. But with respect to human research and applicability to human patients they were out of date by 20 years.

'Miracle' treatments are not the only dangerous for patients and the freedom in research. It is the worldwide research, treatment, and teaching system, that does not allow qualified human research, which is urgently needed to cure diseases and make humans live longer with a better quality of life [19,83]. And if there are really operations with unbelievable success, then the treatment before those operations was wrong.

For example, a paraplegic patient came by wheelchair from a well-known German rehabilitation center to a neurosurgery department, was operated and walked 2 weeks later out of the neurosurgery department. A second patient was cured in the same way. The reason for this progress was caused by a mistake of the rehabilitation center. Bones from the spinal canal caused pressure on the spinal cord (in some similarity to Figure 5A). The spinal cord stopped working but was damaged only little. A laminectomy freed the spinal cord and the spinal cord started to work again. Follow up MRI's are needed which are normally not performed in rehabilitation centers. The argument is that it has no clinical consequence, because the patient is staying anyway in the wheelchair for the rest of his or her life. This argument is wrong since for example cysts can build up in the injured spinal cord which enlarges the spinal cord and pressure symptoms will occur and the patient loses further functions. The injury level may then rise for example from C6 to C5. And, of course, SCI can partly be repaired.

\subsection{Out-of-date of the clinical treatment system}

On the clinical side the human research situation is not better. The diagnostic in clinics is good till sophisticated, because it is organized and money can be earned. Sometimes too much diagnostic is performed. But the cervical SCI patient Kadri (Figure 17) said, why should I go to the neurologist. He is telling me what is wrong in my body, but he is not telling me how to repair the lost or impaired functions. Apart from exceptions, one cannot repair the human nervous system with drugs or operations. Movement-based learning, on the other hand, is a causal therapy which repairs the neural networks. But the physiotherapy to repair the nervous system is mainly out-of-date and inefficient. When in Switzerland the physiotherapy education was upgraded from school to academy, only the names were upgraded, not the education. Physiotherapists do not learn, for example, to perform electromyography (EMG) on patients. When the Author demonstrated surface EMG to physiotherapists in a course, they all liked it, because one could really see pathologic motor programs and spasticity on the screen of the scope. When seeing their own volitional muscle activation, physiotherapists were impressed.

Being at the international conference for pediatric acquired brain injury (IPBIS2018), really interest was only coming from one physiotherapy student who wanted to do research, a physician who was interested in neural network learning and two lawyers, who were supporting and supervising the families of brain injured children. But all the physiotherapists, rehabilitation physicians, neurologists or neuropediatric did not want to get informed about new developments in CNS repair. Robotics were of interest to them. When the Author is seeing a picture where a child with a nervous system injury is in the wheelchair (Figure 32A), he is getting angry and depressive. When the Author asked neuropediatric from New Zeeland whether they are not getting depressed when they diagnose all the deficits of a brain-injured child but cannot offer treatment, he did not get an answer and next day they did not come to the Authors poster (Figure 84) to get informed and discuss problems. The members of the conference seem to be afraid to see that the nervous system of children can partly be repaired. A physician, who treated Nefeli and Sophie (pictured on the lower part of the poster, Figure 84) in a rehabilitation center, did not want to see the outcome of his former patients on the long-term. Interesting is further that no member of the conference wanted to try out 
the special CDT device placed besides the poster (Figure 84). And no physician wanted to get a reprint or wanted to look in some publications or books (Figure 84). When the Author exercised at the entrance of the conference building on the special CDT device, after some time he was pushed away by the administration (the organizer of the conference) so that nobody can see his exercising on the device (out of view - out of mind). Students and stuff of the nearby School for Management, who were passing the Author during exercising on the special CDT device, were more interested what the Author is trying to demonstrate than the members of the conference. When the Author gave long ago a talk at a Nobel institute for Neuroscience in Stockholm (Prof. Grillner [83], pattern generator), he was not allowed to discuss with all the assistances, even though at that time they were thinking of a rat experiment to repair the spinal cord. They were planning at that time a rat experiment what the Author had mainly done already on human.

When really some junior physicians get motivated to try new repair strategies of the brain, they are punished by the establishment. Why to develop new treatment to repair the human brain, when it is impossible to bring it to the patient? Moderators in TV are often open to new developments. But first, explanations have to be that simple that a differentiation between qualified research and hocus-pocus is not possible and secondly, the neurorehabilitation is still going on with their out-of-date treatment whatever the progress is in their field.

New organizations and predatory organizations organize journals and conferences without sufficient knowledge. For both knowledge and money is needed. They screen the market and choose what sounds nice. But they are not blocking new developments. Researcher can now choose between not publishing or publishing in a new journal which may be predatory. But a real research worker has to publish because it is part of the profession and secondly, in human neurophysiology and clinical research, apart from statistics, many patients are suffering and dying and

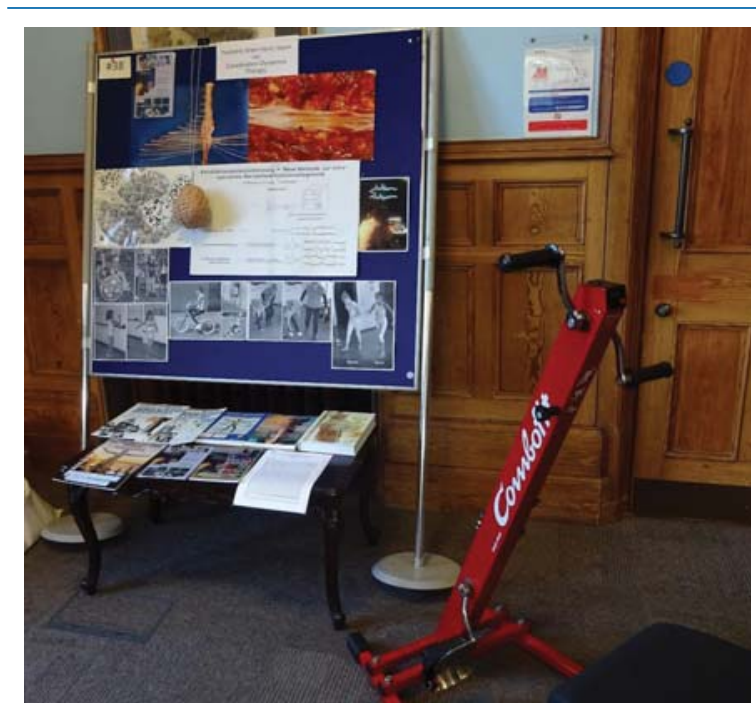

Figure 84: Poster of the Author Schalow G (Number 38) at the international conference IPBIS2018 in Belfast 2018: Pediatric acquired brain injury repair. The poster is not especially good because of lack of money. But the repair progress of the nervous system in children can clearly be seen It is also shown that human anatomy and physiology is needed for repair. that puts load on the clinical researcher. It was estimated that by shifting the new Berlin airport from the South of Berlin to the periphery of Berlin, approximately 11 inhabitants will die per year caused by aircraft noise. By not using CDT, which improves health of patients in general, there may die a million people on earth every year.

Movement therapies are not popular because mental discipline is needed. Probably more than half of the population on earth has overweight. Especially young ones, who alternate between fast food and smart phone, have massive overweight. On Kriti (Greece), there are many children between 5 and 10 years which have already very much overweight and move therefore very little. In the class of Nefeli (Figure 69) there were 2 of the 18 children who had no overweight! But movements like walking, running and jumping are necessary for a healthy development. Many new diseases will therefore occur in the future due to the too little movements performed by children. Patients and healthy people only enjoy movements, if they have no overweight. Also, the elegance of walking is lost with overweight.

Universities and rehabilitation centers are with respect to human neurophysiology approximately 30 years out-of-date. Just now an internet organization (academia.edu) tells what papers of the Author are read most. These papers are the ones which the Author published 10 to 20 years ago (Classification scheme of human peripheral nerve fibers). That the neuro-rehabilitation is out-of-date is generally known. The 
Author was even told that for bringing progress to the rehabilitation an agreement with the devil is justified. But how is it, most likely, with the so-called progressive institutions? Astronauts are brought into space and because of missing gravity it has to be looked for their health. Astronauts get for example osteoporosis from which they do not recover again on earth. Their physical exercise in space is for the time being the walking on treadmill. Exercising on a special CDT device would at least also be needed. One could measure then additionally the organization of their CNS first on earth, then in space and afterwards on earth again and see what had changed of CNS functioning and health in space. By measuring at the same time heart rate variability and split it by Fourier analysis into sympathetic and parasympathetic contributions, one could see whether the astronauts have stress and reduce it, if possible, by exercising. Further, because CDT improves also coordinated finger movements, astronauts could better perform complicated experiments, in which coordination is needed.

Plenty of money is given to enhance artificial intelligence and necessary infrastructure is built. The highest intelligence is still by far generated in the human brain. Why the researchers in the field of artificial intelligence are not trying to learn from the organization of the human neural networks for their artificial neural networks? The human brain is very fast and is most likely not using iteration processes for network changes. It seems that not only disabled children have a "tunnel-view".

Aging men are worried to get prostate cancer. It is likely that prostate cancer growth can be inhibited by CDT in similarity to breast cancer [66]. In TV it was recently analyzed what operational strategy is better, to operate only with the hands or using additionally a robot. But on prevention of prostate cancer, namely to reduce the probability of prostate cancer occurrence by physical activity (including CDT), it was not reported of.

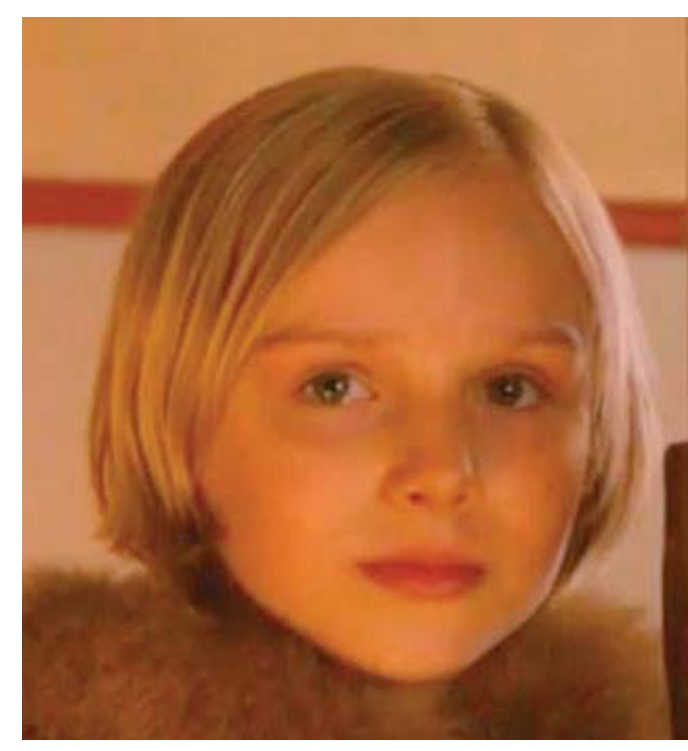

Figure 85: The girl looks at you in the hope that you will help to cure SCI (and other diseases) in children. The picture was taken from the fairy tale film "Till Eulenspiegel". In the film she (Jule Hermann) is hoping that her father wants her.

\subsection{Children with SCI are hoping for better treatment to become like the other children again}

The patient Nefeli was saying to the Author: "Giselher, I want to be again like the other children". The Author answered: "Nefeli, then we have to train very much at the limit". And Nefeli answered: "Giselher, but I also want to play now. When I am grown up, I cannot play anymore".

The girl in Figure 85 is looking at the reader with the hope that she/he is helping to get treatment for children to make them healthy again.

\subsection{Ethics to use the patients name}

The patient Nefeli and her parents agreed to have pictures and Nefeli's name in the book. By demonstrating in detail through movements how Nefeli became better, she wants to help also other children. Children with a CNS injury have psychological stress and need hope. Once, when Nefeli's stress with the SCI was very large, she drew a picture of the surgeon, who made her paraparetic, cut it out and threw it into the fire to reduce her stress in similarity to the fairy tale 'Hänsel und Gretel', where Gretel pushed the witch into the fire.

To have real names in the medical research publications emphasizes its importance. Qualified medical research is connected to real human life and is not just a more or less theoretical game. 


\section{References}

1. Guttmann L (1973) Spinal cord injuries: Comprehensive management and research. Blackwell, Oxford.

2. Schalow G, Jaigma P, Belle VK (2009) Near-total functional recovery achieved in partial spinal cord injury $(50 \%$ injury) after 3 years of coordination dynamics therapy. Electromyogr Clin Neurophysiol 49: 67-91. Link: http://bit. ly/2OW1QF5

3. Schalow G (2019) Brain repair and general health improvement through human neurophysiology and repair physiology. Clinical and Medical Reports 2: 1-68. Link: http://bit.ly/2yCDSDt

4. (2010) Editorials: Order from chaos Nature 466: 7-8.

5. Thuret S, Moon, LDF, Gage FH (2006) Therapeutic interventions after spinal cord injury. Nat Rev Neurosci 7: 628-643. Link: http://bit.ly/2YqBAXn

6. Bradbury EJ, McMahon SB (2006) Spinal cord repair strategies: why do they work? Nat Rev Neurosci 7: 644-653. Link: https://go.nature.com/339ZXYe

7. Schalow $G$ (2002) Recovery from spinal cord injury achieved by 3 months of coordination dynamic therapy. Electromyogr Clin Neurophysiol 42: 367-376. Link: http://bit.ly/33neLTA

8. Schalow G (2003) Partial cure of spinal cord injury achieved by 6 to 13 months of coordination dynamic therapy. Electromyogr Clin Neurophysiol 43: 281-292. Link: http:// bit.ly/2ODZMRK

9. Schalow G (2010) Cure of urinary bladder functions in severe (95\%) motoric complete cervical spinal cord injury in human. Electromyogr. Clin Neurophysiol 50: 155-179. Link: http://bit.ly/2T4yylS

10. Schalow G, Lang G (1987) Recording of Single Unit Potentials in Human Spinal Nerve Roots: a New Diagnostic Tool. Acta Neurochir 86: 25-29. Link: http://bit.ly/2M0qeD2

11. Schalow $G$ (1987) Single unit potential amplitude in relation to the conduction velocity in frog and human. Zent bl Neurochir 48: 109-113. Link: http://bit.ly/2T51mKW

12. Lang G, Schalow G (1988) Experiences with the intraoperative single unit potential recording of spinal cord and cauda equina disorders. Advances in Neurosurgery 16 : 98-99. Link: http://bit.ly/2T735ja

13. Schalow G, Barth $H$ (1992) Group conduction velocities and nerve fibre diameters of $a$ and $y$-motoneurons from lower sacral nerve roots of the dog and humans. Gen Physiol Biophys 11: 85-99. Link: http://bit.ly/2M1iws9

14. Schalow G (1992) Recruitment within the groups of $\gamma_{1}, a_{2}$ and $\mathrm{a}_{3}$-motoneurons in dogs and humans following bladder and anal catheter pulling. Gen Physiol Biophys 11: 101-121. Link: http://bit.ly/2YmS7fb

15. Schalow G (1992) Ventral root afferent and dorsal root efferent fibres in dog and human lower sacral nerve roots. Gen Physiol Biophys 11: 123-131. Link: http://bit.ly/2yBRgl6

16. Schalow G, Zäch GA, Warzock R (1995) Classification of human peripheral nerve fibre groups by conduction velocity and nerve fibre diameter is preserved following spinal cord lesion. J Auton Nerv Syst 52: 125-150. Link: http://bit. ly/31lwV62

17. Schalow G (2013) Human Neurophysiology: Development and Repair of the Human Central Nervous System. Nova Science Publishers Inc Hauppauge NY, USA. Link: http://bit. ly/2KuOFH4

18. Schalow G (2015) Repair of the Human Brain and Spinal Cord. Nova Science Publishers Inc Hauppauge NY, USA.

19. Schalow G (2015) Neural network learning in humans. Nova Science Publishers Inc Hauppauge NY, USA.

20. Schalow G (2005) Tapering of human nerve fibres. Gen Physiol Biophys 24: 427-448. Link: http://bit.ly/2KrxSDb

21. Schöner G, Zanone PG, Kelso JAS (1992) Learning as change of coordination dynamics: Theory and experiment. Journal of Motor Behavior 24: 29-48. Link: http://bit.ly/339kIDj

22. Zanone PG, Kelso JAS (1997) Coordination dynamics of learning and transfer: Collective and component levels. J Exp Psychol Hum Percept Perform 23:1454-1480. Link: http://bit.ly/2OEQaX1

23. Schalow G (2010) Scientific basis for learning transfer from movements to urinary bladder functions for bladder 
repair in patients with spinal cord injury. Electromyogr Clin Neurophysiol 50: 339-395. Link: http://bit.ly/2GMhpZp

24. Kelso JAS (1995) Dynamic Patterns. The Self-Organization of Brain and Behavior. MIT Press, Cambridge. Link: http:// bit.ly/2T6jCDS

25. Haken $H$, Kelso JA, Bunz $H$ (1985) A theoretical model of phase transitions in human hand movements. Biological Cybernetics 51: 347-356. Link: http://bit.ly/2T6hmwg

26. Espinosa-Medina I, Saha O, Boismoreau F, Chettouh Z. Rossi F, et al. (2016) The sacral autonomic outflow is sympathetic. Science 354: 893-897. Link: http://bit.ly/20Eef05

27. Passatore GM, Fillipi GM, Grassi G (1985) Cervical sympathetic nerve stimulation can induce an intrafusal muscle fibre contraction in the rabbit. The Muscle Spindle 221-226. Link: http://bit.ly/2KF3C8b

28. Schalow G, Zäch GA (1996) External loops of human premotor spinal oscillators identified by simultaneous measurements of interspike intervals and phase relations. Gen Physiol Biophys 1: 95-119. Link: http://bit.ly/2ZwOJux

29. Schalow G (2009) Coordination impairment between the somatic and parasympathetic nervous system divisions in the human sacral micturition center following spinal cord injury. Electromyogr Clin Neurophysiol 49: 337-367. Link: http://bit.ly/2M1nfdn

30. Schalow G (2009) The classification and identification of human somatic and parasympathetic nerve fibres including urinary bladder afferents and efferents is preserved following spinal cord injury. Electromyogr Clin Neurophysiol 49: 263-286. Link: http://bit.ly/2KiVATs

31. Schalow G (2010) Phase relation changes between the firings of $a$ and $y$-motoneurons and muscle spindle afferents in the sacral micturition centre during continence functions in brain-dead human and patients with spinal cord injury. Electromyogr Clin Neurophysiol 50: 3-27. Link: http:// bit.ly/2MDKtWB

32. Schalow G (2009) Building of New Motoneurons in the Human Spinal Cord upon Coordination Dynamics Therapy to Improve Finger Functions in Motoric Complete Cervical Spinal Cord Injury. In: Berkovsky, T.C. (Ed.), Handbook of Spinal Cord Injuries, Chapter 4, 231-264. Nova Science Publishers.

33. Schalow G (1993) Action potential patterns of intrafusal $Y$ and parasympathetic motoneurons, secondary muscle spindle afferents and an oscillatory firing $a_{2}$-motoneuron, and the phase relations among them in humans. Electromyogr Clin Neurophysiol 33: 477-503. Link: http:// bit.ly/33fjEOc

34. Baehr M, Frotscher M (2005) Duus' Topical Diagnosis in Neurology. Thieme Verlag, Stuttgart. (a very good book for medical neuroscience since it correlates structure and function in human nervous system injuries).
35. Dauber W (2005) Feneis' Bild-Lexikon der Anatomie. Thieme Verlag, Stuttgart. Link: http://bit.ly/31f3imU

36. Kahle W, Leonhardt H, Platzer W (1975) Taschenatlas der Anatomie, Band 1, Bewegungsapparat (Platzer W.). Thieme Verlag, Stuttgart.

37. Bohm E (1956) Sacral rhizopathies and sacral syndromes (SII-SV). Acta Chirurgica Scandinavia, 216: 5-28.

38. Sramkova T, Skrivanova K, Dolan I, Zamecnik L, Kriz J,et al. (2010) Sexual life after spinal cord injury. In: Berkovsky, T.C. (ed.), Handbook of Spinal Cord Injuries, Nova Science Publishers, New York, 133-166.

39. Courtois F et al. (2010). Assessment of sexual potential and treatment of sexual dysfunctions in men and women with spinal cord injury. In: Berkovsky, T.C. (ed.), Handbook of Spinal Cord Injuries, Nova Science Publishers, New York, 167-229.

40. Ducker TB (1980) Pathophysiology of peripheral nerve trauma. In: Omer, G.E. (ed.), Management of peripheral nerve problems. Saunders, Philadelphia, 475-486.

41. Polezhaev LV (1972) Loss and restoration of regenerative capacity in tissues and organs of animals. Harvard University Press, Cambridge, Massachusetts, 386. Link: http://bit.ly/33dv6de

42. Ducker TB (1980) Metabolic consequences of axotomy and regrows. In: Jewett, D.L. and McCarroll, H.R. (eds.), Nerve repair and regeneration. Its clinical and experimental basis. Mosby, St. Louis, 99-101.

43. Gorio A, Millesi H, Megrino S (1981) Posttraumatic nerve regeneration, experimental basis and clinical implications. Raven Press, New York, 241-242.

44. Jenq CB, Coggeshall RE (1985) Numbers of regenerating axons in parent and tributary peripheral nerves in the rat. Brain Res 326: 27-40. Link: http://bit.ly/2yEzEeF

45. Edds MV, Small WT (1951) The behavior of residual axons in partially denervated muscles of the monkey. $J$ Exp Med 93: 207-215. Link: http://bit.ly/2KrDxsV

46. Edds MV (1953) Collateral nerve regeneration. Q Rev Biol 28: 260-276. Link: http://bit.ly/2T8gzLb

47. Tarlov IM, Berman D, Moldaver J, Kelleman E (1949) Cauda equina regeneration through nerve grafts: an experimental study in monkeys. J Nerv Ment Dis 110: 161-164. Link: http://bit.ly/2T5bcwm

48. Tsukahara N (1978) Synaptic plasticity in the red nucleus. In: Cotman CW (ed.), Neuronal plasticity. Raven Press, New York, 113-130. Link: http://bit.ly/33cTTyk

49. Weiss P, Ruch TC (1936) Further observation on the functions of supernumary fingers in man. Experimental Biology and Medicine 34: 569-570. Link: http://bit.ly/2ZxSOPi 
50. Yu J, Liu CN, Chambers WW, Chen WP, McCouch GP (1981) Effect of exercise on reflexes in paraplegic monkeys. Acta Neurobiol 41: 271-278. Link: http://bit.ly/31hxqyb

51. Lundborg G (1970) Ischemic nerve injury. Scand. J Plastic Reconst Surg 6: 1-113.

52. Gillian LA (1958) The arterial blood supply of the human spinal cord. J Comp Neurol 110: 75-100. Link: http://bit. ly/2YDisRq

53. Gelfan S, Tarlov IM (1955) Differential vulnerability of spinal cord structures to anoxia. J Neurphysiol 18: 170-188. Link: http://bit.ly/31pAw3n

54. Krogh E (1950) The effect of acute hypoxia on the motor cells of the spinal cord. Acta Physiol Scand 20: 263-292. Link: http://bit.ly/2OHdtQ1

55. Gelfan S, Tarlov IM (1959) Interneurons and rigidity of spinal origin. J Physiol 146: 594-617. Link: http://bit.ly/2YDjv80

56. Demian YK, White RJ, Yashon D, Kretchmer HE (1971) Anaesthesia for laminectomy and localized cord cooling in acute cervical spine injury. Br J Anaesth 43: 973-979. Link: http://bit.ly/33d3qFp

57. Gelderd JB (1983) Hyperbaric oxygen and dimethyl sulfoxide therapy following spinal cord injury. In: Kao CC, Bunge RP Reier PJ (eds.), Spinal cord reconstruction. Raven Press, New York, 245-259.

58. Bracken MB, Shepard MJ, Collins WF, Holford TR, Young W, et al. (1990) A randomized controlled trial of methylprednisolone or naloxone in the treatment of acute spinal cord injury. N Engl J Med 322: 1405-1411. Link: http://bit.ly/2yQyOvH

59. Bernstein JJ, Wells MR, Bernstein ME (1978) Spinal cord regeneration: Synaptic renewal and neurochemistry. In: Cotman, CW (ed.), Neuronal plasticity. Raven Press, New York, 49-71.

60. Nesmeyanova TN (1977) Experimental studies in regeneration of spinal neurons. V.H. Winston \& Sons, Washington, Halsted Press Book, John Wiley \& Sons, New York.

61. Sperry RW (1945) The problem of central nervous system reorganization and muscle transposition. Quart Rev Biol 20: 311-369. Link: http://bit.ly/3390YOs

62. Sperry RW (1947) Effect of crossing nerves to antagonistic limb muscles in the monkey. Arch Neurol Psychiat (Chicago) 58: 452-473. Link: http://bit.ly/2YEV7i9

63. Weiss P, Brown PF (1941) Electromyographic study on coordination of leg movements in poliomyelitis patients with transposed tendons. Proc Soc Exper Biol Med 48: 384387.

64. Schalow G (1990) Feeder arteries, longitudinal arterial trunks and arterial anastomoses of the lower human spinal cord. Zentralbl Neurochir 51: 81-184. Link: http://bit.ly/2yDDuon

65. Christensen JF, Jones LW, Andersen JL, Daugaard G, Rorth M, Hojman P (2014) Muscle dysfunction in cancer patients. Ann Oncol 25: 947-958. Link: http://bit.ly/2KfhIDq

66. Schalow G (2017) Breast cancer growth inhibition via Coordination Dynamics Therapy. In: "Horizons in Cancer Research. Volume 68". Editor: Hiroto S. Watanabe. Nova Science Publishers, Inc, Hauppauge NY, USA, 125-151.

67. Schalow G (2019) Permanent coma patient re-learned to speak via Coordination Dynamics Therapy. Arch Clin Med Case Rep 3: 33-50. Link: http://bit.ly/2M4bliT

68. Deisseroth K, Singla S, Toda H, Monje M, Palmer TD, et al. (2004) Excitation-neurogenesis coupling in adult neural stem/progenitor cells. Neuron 42: 535-552. Link: http://bit. ly/2yBg2Z0

69. Colvis CM, Pollok JD, Goodman RH, Impey S, Dunn J, et al. (2005) Epigenetic mechanisms and gene networks in the nervous system. J Neurscience 25: 10379-10379. Link: http://bit.ly/2ZBZAn6

70. Bird A (2007) Perceptions of epigenetics. Nature 447: 396398. Link: http://bit.ly/33hidiD

71. Covic M, Karaca E, Lie DC (2010) Epigenetic regulation of neurogenesis in the adult hippocampus. Heredity 105: 122134. Link: http://bit.ly/2Kqlovv

72. Wattig B, Schalow G, Heydenreich F, Warzok R, CervósNavarro J (1992) Enhancement of nerve fibre regeneration by nucleotides after peripheral nerve crush damage. Electrophysiologic and morphometric investigations. Drug Res 42: 1075-1078. Link: http://bit.ly/2LYyOCp

73. von Holst E (1939) Die relative Koordination als Phänomen zentralnervöser Funktionsanalyse. Ergebnisse der Physiologie, biologischen Chemie und experimentellen Pharmakologie 42: 228-306. Link: http://bit.ly/2yDEFUP

74. Bernstein NA (1967) The Coordination and Regulation of Movement. London, Pergamon Link: http://bit.ly/33agf3k

75. Rosenzweig ES, Courtine G, Jindrich DL, Brock JH, Ferguson $A R$, et al. (2010) Extensive spontaneous plasticity of corticospinal projections after primate spinal cord injury. Nat Neurosci 13: 1505-1510. Link: http://bit.ly/2yDgA0z

76. Maier IC, Schwab ME (2006) Sprouting and circuit formation in the injured spinal cord: factors and activity. Philos. Trans. R. Soc Lond B Biol Sci 361: 1611-1634. Link: http://bit. ly/2YKgumf

77. Zorner B, Schwab ME (2010) Anti - Nogo on the go: from animal models to a clinical trial. Ann N Y Acad Sci 1198 Suppl1: E22-34. Link: http://bit.ly/2YDOMFu

78. Keirstead HS, Nistor G, Bernal G, Totoiu M, Cloutier F, et al. (2005) Human embryonic stem cell derived oligodendrocyte

Giselher Schalow, Switzerland ISBN: 978-81-943057-0-5 
progenitor cell transplants remyelinate and restore locomotion after spinal cord injury. J Neurosci 25: 46944705. Link: http://bit.ly/2Yld75Q

79. Martino G, Pluchino S (2006) The therapeutic potential of 82. Har neural stem cells. Nat Rev Neurosci 7: 395-406. Link: http:// bit.ly/20Fwm5S

80. McDonald JW, Liu XZ, Qu Y, Liu S, Mickey SK, et al. (1999) Transplanted embryonic stem cells survive, differentiate and promote recovery in injured rat spinal cord. Nat Med 5: 1410-1412. Link: http://bit.ly/2M26X4a

81. Kerschensteiner $M$, Schwab ME, Lichtman JW, Misgeld
T (2005) In vivo imaging of axonal degeneration and regeneration in the injured spinal cord. Nat Med 11: 572577. Link: http://bit.ly/31gl1dO recapitulate developmental guidance during recovery from spinal cord injury. Nat Rev Neurosci 7: 603-616. Link: http:// bit.ly/2YLA9IY

83. Grillner S (2003) The motor infrastructure: From ion channels to neuronal networks. Nat Rev Neurosci 4: 573586. Link: http://bit.ly/20Elx3X

Discover a bigger Impact and Visibility of your article publication with

\section{Peertechz Publications}

\section{Highlights}

* Signatory publisher of ORCID

* Signatory Publisher of DORA (San Francisco Declaration on Research Assessment)

* Articles archived in worlds' renowned service providers such as Portico, CNKI, AGRIS, TDNet, Base (Bielefeld University Library), CrossRef, Scilit, J-Gate etc.

* Journals indexed in ICMJE, SHERPA/ROMEO, Google Scholar etc.

* OAI-PMH (Open Archives Initiative Protocol for Metadata Harvesting)

* Dedicated Editorial Board for every journal

* Accurate and rapid peer-review process

* Increased citations of published articles through promotions

* Reduced timeline for article publication

Submit your articles and experience a new surge in publication services (https://www.peertechz.com/submission). 
